\title{
A GODEMENT-JACQUET TYPE INTEGRAL AND THE METAPLECTIC SHALIKA MODEL
}

\author{
EYAL KAPLAN AND JAN MÖLLERS
}

\begin{abstract}
We present a novel integral representation for a quotient of global automorphic $L$-functions, the symmetric square over the exterior square. The pole of this integral characterizes a period of a residual representation of an Eisenstein series. As such, the integral itself constitutes a period, of an arithmetic nature. The construction involves the study of local and global aspects of a new model for double covers of general linear groups, the metaplectic Shalika model. In particular, we prove uniqueness results over $p$-adic and Archimedean fields, and a new Casselman-Shalika type formula.
\end{abstract}

\section{INTRODUCTION}

Let $\mathbb{A}$ be the ring of adèles of a global field and let $\pi$ be a cuspidal automorphic representation of $\mathrm{GL}_{k}(\mathbb{A})$. One of the pillars of the Langlands program is the study of global automorphic $L$-functions, as mediating agents in the framework of functoriality. Integral representations are an indispensable tool in this study. Godement and Jacquet [GJ72] constructed the integral representation of the standard $L$-function of $\pi$. Jacquet, PiatetskiShapiro and Shalika developed Rankin-Selberg integrals for $\mathrm{GL}_{k} \times \mathrm{GL}_{m}$. Numerous authors considered integral representations for a product of a classical group and $\mathrm{GL}_{m}$, e.g., GPSR87, Gin90, Sou93, GPSR97, GRS98, to name a few.

In several settings, Rankin-Selberg integrals offer a "bonus", namely a period integral characterizing the pole or central value of the $L$-function. For example, the pole of the exterior square $L$-function at $s=1$ was characterized by the nonvanishing of a global Shalika period [JS90]. A similar result was obtained for the symmetric square $L$-function by Bump and Ginzburg [BG92. Periods of automorphic forms have been studied extensively, e.g., GRS99a, GRS99b, GJR04, and they are especially interesting when they are of an arithmetic nature [GJR04.

We present a new integral representation and use it to characterize the nonvanishing of a co-period of an Eisenstein series. Let $f$ be a global matrix coefficient of $\pi$, and $\varphi$ and $\varphi^{\prime}$ a pair of automorphic forms in the space of the exceptional representation of a double cover of $\mathrm{GL}_{n}(\mathbb{A}), n=2 k$. The cover and the exceptional representation were constructed by Kazhdan and Patterson [KP84]. We define a zeta integral $Z\left(f, \varphi, \varphi^{\prime}, s\right)$ (see $\S$ [5.2), where we integrate $f$ against the Fourier coefficients of $\varphi$ and $\varphi^{\prime}$ along the Shalika unipotent subgroup and its (Shalika) character. Technically, it resembles the Godement-Jacquet zeta integral, where a matrix coefficient was integrated against a Schwartz-Bruhat function GJ72, but they are not to be confused - our integral is not an entire function. It is absolutely convergent in some right half-plane, and for decomposable data becomes Eulerian. The computation of the resulting local integrals with unramified data lets us identify the $L$-functions represented.

2010 Mathematics Subject Classification. Primary 11F70; Secondary 11F27.

Key words and phrases. L-functions, co-period integral, periods, Shalika model, covering groups. 
We obtain the meromorphic continuation from the known properties of these $L$-functions, and a detailed analysis of the local integrals.

Theorem A (see Theorem 5.4). The zeta integral extends to a meromorphic function on the plane, and represents the quotient of partial L-functions

$$
\frac{L^{S}\left(2 s, \mathrm{Sym}^{2}, \pi\right)}{L^{S}\left(2 s+1, \wedge^{2}, \pi\right)} .
$$

The corresponding local integrals are studied at both $p$-adic and Archimedean places in $\S$ 5.1. In light of existing Rankin-Selberg constructions, the denominator is expected to arise from the normalization of an Eisenstein series on $\mathrm{SO}_{2 n}$. This is not evident at present (see the discussion below). On the other hand, the presence of exceptional representations and a cover of $\mathrm{GL}_{n}$ in an integral representing the symmetric square $L$-function is well understood, considering the role these played in the Rankin-Selberg integral of Bump and Ginzburg [BG92, and in its extension to the twisted symmetric square $L$-function by Takeda [Tak14].

The symmetric square $L$-function has been studied extensively, both via the LanglandsShahidi method by Shahidi (e.g., [Sha81, Sha88, Sha90]), and the Rankin-Selberg method by Bump and Ginzburg [BG92. The present zeta integral is weaker than these works, in the sense that it does not seem to yield a global functional equation. At least at $p$-adic places, local multiplicity-one results are known (proved in Kap15a]), then any "similar" type of integral may be used to define a functional equation with a proportionality factor. However, for this to be useful the local proportionality factors should also be related to a global one. See Remark 5.2 for more details. On the other hand, we can place the global integral in a different framework, that of periods.

The notion of co-period integrals was introduced by Ginzburg, Jiang and Rallis [GJR01, for the purpose of characterizing the zero of the symmetric cube $L$-function of a cuspidal automorphic representation of $\mathrm{GL}_{2}(\mathbb{A})$ at $1 / 2$, using the spectral decomposition of a tensor of two exceptional representations of the 3 -fold cover of $\mathrm{GL}_{2}(\mathbb{A})$. Their co-period was an integral of an Eisenstein series against two automorphic forms in the space of an exceptional representation of the 3 -fold cover of the exceptional group $\mathrm{G}_{2}(\mathbb{A})$. A co-period involving an Eisenstein series and two automorphic forms in the space of an exceptional representation of a cover of $\mathrm{SO}_{2 n+1}(\mathbb{A})$ (or $\operatorname{GSpin}_{2 n+1}(\mathbb{A})$ ) was analyzed in Kap15b, Kap16 (following GJS10]).

In an ongoing work, Shunsuke Yamana and the first named author study a co-period integral on $\mathrm{GL}_{n}(\mathbb{A})$. Assume that $\pi$ is self-dual with a trivial central character. Let $E(g ; \rho, s)$ be an Eisenstein series corresponding to a vector $\rho$, of the representation parabolically induced from $\pi \otimes \pi^{\vee}$ to $\mathrm{GL}_{n}(\mathbb{A})$, such that the residue $E_{1 / 2}(g ; \rho)$ of the series at $s=1 / 2$ is related to the pole of $L(s, \pi \times \pi)$ at $s=1$ (in fact, a more general setting is studied). The global co-period integral $C P\left(E_{1 / 2}(\cdot ; \rho), \varphi, \varphi^{\prime}\right)$ is an integral of the residue against $\varphi$ and $\varphi^{\prime}$ (see $\S$ 6.1). The integral lends itself to a close scrutiny using the truncation operator of Arthur Art78, Art80]. Shunsuke Yamana and the first named author proved that this co-period is nonvanishing if and only if $L^{S}\left(s, \mathrm{Sym}^{2}, \pi\right)$ has a pole at $s=1$. Moreover, the co-period was unfolded to an explicit "outer period", resembling the integral of the symmetric square $L$-function of [BG92].

It turns out that, using a different analysis and under a mild assumption regarding the Archimedean places (see Assumption [5.5), we can obtain a more precise "outer period". Namely, we prove: 
Theorem B (see Theorem 6.2). $C P\left(E_{1 / 2}(\cdot ; \rho), \varphi, \varphi^{\prime}\right)=\int_{K} \operatorname{Res}_{s=1 / 2} Z\left(f_{k \rho}, k \varphi, k \varphi^{\prime}, s\right) d k$, where $K$ is the standard (global) compact subgroup and $f_{k \rho}$ is a matrix coefficient on $\pi$. Moreover, the co-period is not identically zero if and only if the zeta integral has a nontrivial residue at $s=1 / 2$.

The results on the co-period have a local $p$-adic counterpart, proved in Kap15a. Let $\tau$ be a self-dual irreducible supercuspidal representation of $\mathrm{GL}_{k}$. The results of Kap15a imply that if the symmetric square $L$-function of $\tau$ has a pole at $s=0$, the Langlands quotient of the representation parabolically induced from $|\operatorname{det}|^{1 / 2} \tau \otimes|\operatorname{det}|^{-1 / 2} \tau$ to $\mathrm{GL}_{n}$ has a nontrivial bilinear $\mathrm{GL}_{n}$ pairing with a tensor of exceptional representations.

Our zeta integral involves a new model for double covers of $\mathrm{GL}_{n}$, the metaplectic Shalika model, first discovered in Kap15a during the computation of twisted Jacquet modules of the $p$-adic exceptional representation (of [KP84]). The Jacquet module of this representation along the Shalika unipotent subgroup and character is one-dimensional, and the reductive part of its stabilizer acts by a Weil factor. Therefore, this representation admits a unique embedding in a space of metaplectic Shalika functions. The key observation underlying this model for the cover, is that the restriction of the cover to the Shalika group is simple, in the sense that the cocycle is given by the quadratic Hilbert symbol. This follows immediately from the block-compatibility of the cocycle of Banks, Levy and Sepanski [BLS99]. See $\S 3.1$ for a precise introduction to this model.

Roughly speaking, a model of a representation is an embedding, preferably unique, into a space of functions on the group, which is beneficial and convenient in terms of applications. One important model is the Whittaker model, which has had a profound impact on the study of representations, with a vast number of applications, perhaps most notably the LanglandsShahidi theory of local coefficients. This model has a natural extension to covering groups, because unipotent subgroups are split under the cover. Indeed, the metaplectic Whittaker model has been studied, e.g., in [KP84, BFH91, McN11, CO13]. The main downside to using this model for covering groups, is that multiplicity one no longer holds. For instance over an $r$-fold cover of $\mathrm{GL}_{n}$, the dimension of the space of Whittaker functionals on an unramified principal series representation is essentially $r^{n}$.

The (non-metaplectic) Shalika model was first introduced in a global context in the aforementioned work of Jacquet and Shalika [JS90]. Friedberg and Jacquet [FJ93] used it to characterize cuspidal automorphic representations affording linear models, in terms of the pole of the exterior square $L$-function at $s=1$ (see also [BF90]). Ash and Ginzburg [AG94] used this model to construct $p$-adic $L$-functions. Multiplicity one results were proved by Jacquet and Rallis [JR96] (over $p$-adic fields) and by Aizenbud, Gourevitch and Jacquet [AGJ09] over Archimedean fields. Globally, a cuspidal automorphic representation $\pi$ affords a Shalika functional (given by an integral) if and only if $L^{S}\left(s, \wedge^{2}, \pi\right)$ has a pole at $s=1$, and equivalently is a weak functorial lift from $\mathrm{SO}_{2 n+1}$ ([JS90, GRS99b, GRS01]). A similar local result was proved for supercuspidal representations in [JNQ08, and is expected to hold in greater generality. Further studies and applications of this model include GRS99a, JQ07, JNQ10.

In this work we launch a study of the metaplectic Shalika model. In sharp contrast to the Whittaker model, we conjecture that the metaplectic Shalika model does enjoy multiplicity one. We establish the following result.

Theorem C (see Theorems 3.10 and 3.37). Let $\mathrm{I}(\chi)$ be a principal series representation of a double cover of $\mathrm{GL}_{n}$, over any local field, but over a p-adic field assume it is unramified. 
Assume $\chi$ satisfies a certain regularity condition, which does not preclude reducibility. Then the space of metaplectic Shalika functionals on $\mathrm{I}(\chi)$ is at most one-dimensional. Over a $p$-adic field and when $\mathrm{I}(\chi)$ is irreducible, this space is one-dimensional if and only if $\mathrm{I}(\chi)$ is a "lift" from a classical group.

It is perhaps premature to discuss lifting problems for covering groups; here we simply mean the natural condition one expects $\chi$ to satisfy, analogous to the case of $\mathrm{GL}_{n}$. This result is our main tool to deduce that the (global) zeta integral is Eulerian.

Another practical starting point for the study of the metaplectic Shalika model, is the unramified setting. We prove a Casselman-Shalika formula for an unramified principal series representation of a double cover of $\mathrm{GL}_{n}$. For $\mathrm{GL}_{n}$ this formula was proved by Sakellaridis [Sak06], and our proof closely follows his arguments.

Theorem D (see Theorem 3.28 and Corollary 3.35). Let $\mathrm{I}(\chi)$ be an unramified principal series representation of a double cover of $\mathrm{GL}_{n}$, which admits a metaplectic Shalika model. There is an explicit formula for the values of the unique unramified normalized function in the model, in terms of the character $\chi$.

We apply our formula to the exceptional representation and use it to compute the local zeta integrals with unramified data.

In his proof, Sakellaridis Sak06] used a variant of the Casselman-Shalika method developed by Hironaka [Hir99, Proposition 1.9]. The original approach of Casselman and Shalika [CS80] was to compute functionals on torus translates of the Casselman basis element corresponding to the longest Weyl element. A somewhat more versatile approach has been introduced by Hironaka, namely to compute the projection of the functional, say, the Shalika functional, onto the Iwahori invariant subspace. So, our first step is to extend some of the ideas from [Hir99] to the cover. Hironaka established a formula for expressing a spherical function on certain spherical homogeneous spaces, which is applicable also in the absence of uniqueness results. We verify this formula for the double cover of $\mathrm{GL}_{n}$, see Lemma 2.15 and Corollary 2.17. In fact, our proof is general and applicable to a large class of covering groups, e.g., the central extensions of unramified reductive $p$-adic groups by finite cyclic groups, studied by McNamara [McN11, McN12, McN14] (at present, with his assumption on the cardinality of the residue field). See the discussion in the end of $\S 2.3$.

Since the formula of [Hir99] does not depend on a uniqueness property, it may well be more adequate for covering groups, where multiplicity one results may fail (e.g., for the Whittaker model). Our analog of [Hir99, Proposition 1.9] involves the verification of several results well known in the non-metaplectic setting. We greatly benefited from the work of Chinta and Offen [CO13, explaining how to extend the results of Casselman Cas80] to $(r$-fold) covers of $\mathrm{GL}_{n}$. We must point out that the study of spherical functions on spherical varieties is vigorously expanding. The aforementioned techniques (e.g., of [Hir99]) have been extended and generalized by Sakellaridis Sak13. While his work does not include covering groups, his ideas are expected to apply to this context, albeit with some modifications.

As mentioned above, the Shalika model has global aspects and the same applies to its metaplectic analog. In a global setting, as with the Whittaker model, one requires a (perhaps) stronger notion. An automorphic representation is called globally generic if it admits a Whittaker functional given by a Fourier coefficient. Surprisingly enough, we prove:

Theorem E (see Theorem 4.22). The global exceptional representation admits a metaplectic Shalika functional, given by a Fourier coefficient. 
Using this, we prove that exceptional representations over Archimedean fields also admit a metaplectic Shalika model, which is then unique, because of Theorem C. Theorem E also plays a key role in the definition of the zeta integral. Moreover, we study a family of Fourier coefficients of exceptional representations and use their properties to prove Theorem B.

Theorem E demonstrates a very special phenomenon, although not estranged from exceptional representations of other groups (see, e.g., [Kap15b, $\S 3.4 .2]$ ). In fact, it is intimately related to the fact that minimal, or small representations are supported on small unipotent orbits, in the sense that generic Fourier coefficients of their automorphic forms vanish on sufficiently large (with respect to the partial ordering) unipotent orbits. See Car93, CM93, BFG03, Gin06, for more details. We mention that Shalika functionals written solely in terms of a unipotent integration already appeared in a work of Beineke and Bump [BB06] in the non-metaplectic case, for a specific representation.

As mentioned above, the proof of meromorphic continuation of the global zeta integrals involves establishing meromorphic continuation of the local integrals. Over Archimedean fields, such a result is usually difficult to obtain, the analysis must be performed carefully, due to the involvement of topological vector spaces. To achieve this, we develop an asymptotic expansion of metaplectic Shalika functions over Archimedean fields, using tools and techniques from Wallach [Wal83, Wal88, Wal92] and Soudry [Sou95, see $\S$ [3.2. This result may be of independent interest (it also applies to the non-metaplectic setting). Note that as a preliminary result, we write an asymptotic expansion of smooth matrix coefficients, on a whole Weyl chamber. This result applies to any real reductive group.

The rest of this work is organized as follows. In $\S 1$ we establish some notation and preliminaries, and recall several facts about the double cover. Section 2 is dedicated to $p$ adic unramified theory. Section 3 begins with a general discussion of the metaplectic Shalika model, then we compute the Casselman-Shalika formula.

In $\S 4$ we discuss exceptional representations. We briefly recall their definition in $\S 4.1$. Local results are contained in $\S[4.2$, where we apply Theorem D to unramified exceptional representations, and Theorem $\mathrm{C}$ to exceptional representations over $\mathbb{R}$. In $\oint 4.3$ we study global Fourier coefficients. The local and global theory of the zeta integral is contained in $\S$ 5. Section [6 is devoted to the co-period.

Acknowledgments. The first named author wishes to express his gratitude to David Ginzburg, Erez Lapid and David Soudry for many encouraging conversations. We thank Yiannis Sakellaridis for his interest in Theorem D and useful remarks. We thank Dmitry Gourevitch and Nolan Wallach for helpful correspondences. Lastly, the authors are grateful to Jim Cogdell and Robert Stanton for their kind encouragement and helpful remarks.

\section{Contents}

Introduction

1. Preliminaries

2. Metaplectic Hironaka Theory

3. The metaplectic Shalika model

4. Metaplectic Shalika model for exceptional representations

5. A Godement-Jacquet type integral

6. The co-period

References 


\section{Preliminaries}

1.1. The groups. Let $G_{n}=\mathrm{GL}_{n}$. Fix the Borel subgroup $B_{n}=T_{n} \ltimes N_{n}$ of upper triangular matrices in $G_{n}$, where $T_{n}$ is the diagonal torus. For any $k \geq 0$, let $Q_{k}=M_{k} \ltimes U_{k}$ be the standard maximal parabolic subgroup of $G_{n}$, whose Levi part $M_{k}$ is isomorphic to $G_{k} \times G_{n-k}$. For any parabolic subgroup $Q, \delta_{Q}$ denotes its modulus character. If $U$ is a unipotent radical of a standard parabolic subgroup, let $U^{-}$denote the unipotent radical opposite to $U$.

Let $W$ be the Weyl group of $G_{n}$. The longest Weyl element is denoted by $w_{0}$ and the identity element by $e$. For $w \in W$, let $\ell(w)$ be the length of $w$. Let $\Sigma_{G_{n}}$ be the set of roots, $\Sigma_{G_{n}}^{+}$be the subset of positive roots and $\Delta_{G_{n}}$ be the set of simple roots compatible with our choice of $B_{n}$. For $\alpha \in \Delta_{G_{n}}$, denote the simple reflection along $\alpha$ by $s_{\alpha}$. Of course $W$ is generated by the set of simple reflections.

In general for any group $G, C_{G}$ denotes its center. If $Y<G$ and $d \in \mathbb{Z}, Y^{d}=\left\{y^{d}: y \in Y\right\}$. Also for $x, y \in G,{ }^{x} y=x y x^{-1},{ }^{x} Y=\left\{{ }^{x} y: y \in Y\right\}$.

Throughout, all fields will be assumed to have characteristic different from 2. If $F$ is a local field, we usually denote $G_{n}=G_{n}(F)$. For a global field $F$, its ring of adèles is denoted A. The vector space of $k \times k$ matrices over a ring $R$ (usually a local field) is denoted $R_{k \times k}$.

In the Archimedean case we use small gothic letters like $\mathfrak{g}_{n}$ and $\mathfrak{u}_{k}$ to denote the Lie algebras of the corresponding groups $G_{n}$ and $U_{k}$. For $F=\mathbb{C}$ we regard the complex Lie algebras as real ones. Write $\mathcal{U}\left(\mathfrak{g}_{n}\right)$ for the universal enveloping algebra of the complexification of $\mathfrak{g}_{n}$ and $\mathcal{Z}\left(\mathfrak{g}_{n}\right)$ for its center.

1.2. The local metaplectic cover. Let $F$ be a local field, $(\cdot, \cdot)_{2}$ be the Hilbert symbol of order 2 of $F$, and $\mu_{2}=\{-1,1\}$. Put $G_{n}=G_{n}(F)$. Kazhdan and Patterson [KP84] constructed the double cover $\widetilde{G}_{n}$ of $G_{n}$, using the double cover of $\mathrm{SL}_{n+1}$ of Matsumoto [Mat69] and the embedding $g \mapsto \operatorname{diag}\left(g\right.$, $\left.\operatorname{det} g^{-1}\right)$ of $G_{n}$ in $\mathrm{SL}_{n+1}$.

Given a 2-cocycle $\sigma: G_{n} \times G_{n} \rightarrow \mu_{2}$, let $\widetilde{G}_{n}$ be the associated central extension of $G_{n}$ by $\mu_{2}, p: \widetilde{G}_{n} \rightarrow G_{n}$ be the natural projection and $\mathfrak{s}: G_{n} \rightarrow \widetilde{G}_{n}$ be a section such that $p(\mathfrak{s}(g))=g$ and $\sigma\left(g, g^{\prime}\right)=\mathfrak{s}(g) \mathfrak{s}\left(g^{\prime}\right) \mathfrak{s}\left(g g^{\prime}\right)^{-1}$ (we also require $\mathfrak{s}$ to take the identity element of $G_{n}$ to the identity element of $\left.\widetilde{G}_{n}\right)$. For any subset $X \subset G_{n}$, put $\widetilde{X}=p^{-1}(X)$.

We use the block-compatible cocycle $\sigma=\sigma_{n}$ of Banks, Levi and Sepanski [BLS99]. The block-compatibility formula is given by

$$
\sigma\left(\operatorname{diag}(a, b), \operatorname{diag}\left(a^{\prime}, b^{\prime}\right)\right)=\sigma_{k}\left(a, a^{\prime}\right) \sigma_{n-k}\left(b, b^{\prime}\right)\left(\operatorname{det} a, \operatorname{det} b^{\prime}\right)_{2}, \quad(a, b),\left(a^{\prime}, b^{\prime}\right) \in M_{k} .
$$

This cocycle is trivial when $n=1$, and for $n=2$ coincides with the cocycle of Kubota Kub67]. Specifically if $g=\left(\begin{array}{ll}a & b \\ c & d\end{array}\right) \in G_{2}$, put $\mathbf{x}(g)=c$ if $c \neq 0$, otherwise $\mathbf{x}(g)=d$, then

$$
\sigma\left(g, g^{\prime}\right)=\left(\frac{\mathbf{x}\left(g g^{\prime}\right)}{\mathbf{x}(g)}, \frac{\mathbf{x}\left(g g^{\prime}\right)}{\mathbf{x}\left(g^{\prime}\right) \operatorname{det} g}\right)_{2} .
$$

The cocycle $\sigma$ and its twist

$$
\sigma^{(1)}\left(g, g^{\prime}\right)=\left(\operatorname{det} g, \operatorname{det} g^{\prime}\right)_{2} \sigma\left(g, g^{\prime}\right)
$$

exhaust the nontrivial cohomology classes. All of our results in this work hold for both $\sigma$ and $\sigma^{(1)}$. In fact, all of the computations will involve $g$ and $g^{\prime}$ such that $\left(\operatorname{det} g \text {, } \operatorname{det} g^{\prime}\right)_{2}=1$ (e.g., $\operatorname{det} g \in F^{* 2}$ ).

We mention several properties of $\sigma$ (and $\sigma^{(1)}$ ), that will be applied throughout. For any $g \in G_{n}$ and $v \in N_{n}, \mathfrak{s}(g v)=\mathfrak{s}(g) \mathfrak{s}(v)$ and $\mathfrak{s}(v g)=\mathfrak{s}(v) \mathfrak{s}(g)$. If ${ }^{g} v \in N_{n}$, then $\mathfrak{s}\left({ }^{g} v\right)=$ $\mathfrak{s}(g) \mathfrak{s}(v) \mathfrak{s}(g)^{-1}$. Consequently if $\mathfrak{s}$ is a splitting of $M<G_{n}$ (i.e., a homomorphism), $U<N_{n}$ 
and $M U=M \ltimes U, \mathfrak{s}$ is a splitting of $M U$. Note that $C_{\widetilde{G}_{n}}=\widetilde{C}_{G_{n}}^{\mathrm{e}}$, where $\mathrm{e}=1$ if $n$ is odd, otherwise $\mathrm{e}=2$.

For computations we fix a concrete set of elements $\mathfrak{W} \subset G_{n}$ as in [BLS99], which is in bijection with $W$. For $\alpha \in \Sigma_{G_{n}}$, let $w_{\alpha}$ be the image of $\left({ }_{1}{ }^{-1}\right)$ in the subgroup of $G_{n}$ corresponding to $\alpha$. Define

$$
\mathfrak{W}=\left\{w_{\alpha_{1}} \cdot \ldots \cdot w_{\alpha_{\ell(w)}}: w \in W, w=s_{\alpha_{1}} \cdot \ldots \cdot s_{\alpha_{\ell(w)}}\right\} .
$$

1.3. The global metaplectic cover. Let $F$ be a global field. For a finite place $v$ of $F$, let $\mathcal{O}_{v}$ denote the ring of integers of $F_{v}$ and $\varpi_{v}$ be a generator of the maximal ideal. The group $G_{n}(\mathbb{A})$ is the restricted direct product $\prod_{\nu}^{\prime} G_{n}\left(F_{\nu}\right)$ with respect to the compact subgroups $\left\{K_{v}\right\}_{v}$, where $K_{v}=G_{n}\left(\mathcal{O}_{v}\right)$ for almost all $v$, and we denote $K=\prod_{v} K_{v}$. The global double cover $\widetilde{G}_{n}(\mathbb{A})$ was constructed in [KP84, $\oint$ 0.2] (see also [FK86, Tak14]). For all $v<\infty$ such that $q_{v}=\left|\varpi_{v} \mathcal{O}_{v} \backslash \mathcal{O}_{v}\right|$ is odd and $q_{v}>3$, there are canonical splittings $\kappa_{v}$ of $K_{v}$ ([Moo68, pp. 54-56], see also [KP84, Proposition 0.1.3]). We extend them to sections of $G_{n}\left(F_{v}\right)$. Also set $\mu_{2}^{\times}=\left\{\left(\epsilon_{v}\right)_{v} \in \prod_{v}^{\prime} \widetilde{G}_{n}\left(F_{v}\right): \epsilon_{v} \in \mu_{2}, \prod_{v} \epsilon_{v}=1\right\}$. Then $\widetilde{G}_{n}(\mathbb{A})=\mu_{2}^{\times} \backslash \prod_{\nu}^{\prime} \widetilde{G}_{n}\left(F_{\nu}\right)$, where the restricted direct product is taken with respect to $\left\{\kappa_{v}\left(K_{v}\right)\right\}_{v}$. There is a global function $\mathfrak{s}=\prod_{v} \mathfrak{s}_{v}$, which is not defined on $G_{n}(\mathbb{A})$, but is a splitting of $G_{n}(F)$ and $N_{n}(\mathbb{A})$ and is well defined on $T_{n}(\mathbb{A})$. We identify $G_{n}(F)$ and $N_{n}(\mathbb{A})$ with their image under $\mathfrak{s}$ in $\widetilde{G}_{n}(\mathbb{A})$.

1.4. Local representations. Let $\operatorname{Alg} G$ be the category of complex and smooth representations of $G$, if $G$ is an l-group (in the sense of [BZ76]), or smooth admissible Fréchet representations of moderate growth if $G$ is a real reductive group.

For $\pi \in \operatorname{Alg} G$ we denote by $\pi^{\vee}$ the contragredient representation of $\pi$. If $V$ is the space of $\pi, V^{\vee}$ will denote the space of $\pi^{\vee}$. For a real reductive group $G, \pi^{\vee}$ is the smooth admissible Fréchet globalization of moderate growth, of the $K$-finite vectors in the dual representation. Then a matrix coefficient of $\pi$ is a function of the form $f(g)=\xi^{\vee}(\pi(g) \xi)$ where $\xi \in V$ and $\xi^{\vee} \in V^{\vee}$.

Regular induction is denoted Ind, and ind is the compact induction. Induction is not normalized. Over an Archimedean field induction denotes smooth induction.

For an $l$-space or an abelian Lie group $X$, let $\mathcal{S}(X)$ be the space of Schwartz-Bruhat functions on $X$.

Let $G$ be an $l$-group and $\pi \in \operatorname{Alg} G$. Let $U<G$ be a closed subgroup, exhausted by its compact subgroups (here $U$ will be a unipotent subgroup of $G_{n}$ ), $\psi$ be a character of $U$, and $M<G$ be the normalizer of $U$ and stabilizer of $\psi$. The Jacquet module of $\pi$ with respect to $U$ and $\psi$ is denoted $\pi_{U, \psi}$. It is a representation of $M$. The action is not normalized. The kernel of the surjection $\pi \rightarrow \pi_{U, \psi}$ is denoted $\pi(U, \psi)$, and occasionally we write the elements of $\pi_{U, \psi}$ in the form $\xi+\pi(U, \psi)$ ( $\xi$ in the space of $\pi$ ). When $\psi=1$, we simply write $\pi_{U}$ and $\pi(U)$.

Let $\pi \in \operatorname{Alg} G_{n}$ be irreducible and $Q=M \ltimes U$ be a parabolic subgroup of $G_{n}$. Assume that the underlying field is $p$-adic. The normalized exponents of $\pi$ along $Q$ are the central characters of the irreducible constituents of $\delta_{Q}^{-1 / 2} \pi_{U}$. For example if $\pi$ is an irreducible representation with a unitary central character, then it is square integrable if and only if its normalized exponents along any standard parabolic subgroup lie in the open cone spanned by the positive roots in $M$.

1.5. Asymptotic expansions over Archimedean fields. We derive an asymptotic expansion for matrix coefficients $f(g)=\xi^{\vee}(\pi(g) \xi)$ of $G_{k}$. The key ideas are due to Wallach 
(see [Wal83, Theorem 7.2], Wal88, Chapters 4.3, 4.4] and [Wal92, Chapter 15.2]), we adapt them to our setting (see also [Sou95, Theorem 1 in $\S 4]$ ). The novelty is that we derive an expansion into an exponential power series, not only on a one-parameter group but on a whole Weyl chamber, depending continuously on $\xi$ and $\xi^{\vee}$. We carry out the details for $G_{k}$, but the proof extends to any real reductive group.

Let $F$ be an Archimedean field. Recall the maximal parabolic subgroups $Q_{\ell}=M_{\ell} \ltimes U_{\ell}$ of $G_{k}$. The non-compact central factor of $M_{\ell}$ is given by $A_{\ell}=\exp \left(\mathfrak{a}_{\ell}\right)$ with $\mathfrak{a}_{\ell}=\mathbb{R} I_{k}+\mathbb{R} H_{\ell}$ and $H_{\ell}=\operatorname{diag}\left(I_{\ell}, 0_{k-\ell}\right)$. For $x=\left(x_{1}, \ldots, x_{k}\right) \in \mathbb{R}^{k}$ put $a_{x}=\exp \left(-\sum_{\ell=1}^{k} x_{\ell} H_{\ell}\right)$.

For a representation $\pi \in \operatorname{Alg} G_{k}$ on a space $V$, we write $E_{1}\left(Q_{\ell}, \pi\right) \subset\left(\mathfrak{a}_{\ell}\right)_{\mathbb{C}}^{*}$ for the finite set of generalized $\mathfrak{a}_{\ell}$-weights in $V / \mathfrak{u}_{\ell} V$. Put

$$
\begin{aligned}
& E_{1}^{(\ell)}(\pi)=\left\{\mu\left(H_{\ell}\right): \mu \in E_{1}\left(Q_{\ell}, \pi\right)\right\} \subset \mathbb{C}, \\
& E^{(\ell)}(\pi)=E_{1}^{(\ell)}(\pi)+\mathbb{N}, \\
& \Lambda_{\pi, \ell}=\min \left\{\operatorname{Re} z: z \in E^{(\ell)}(\pi)\right\}=\min \left\{\operatorname{Re} z: z \in E_{1}^{(\ell)}(\pi)\right\}, \\
& \Lambda_{\pi}=\left(\Lambda_{\pi, 1}, \ldots, \Lambda_{\pi, k}\right) .
\end{aligned}
$$

Here $\mathbb{N}=\{0,1,2, \ldots\}$. By Wal92, Theorem 15.2.4] there exist continuous seminorms $q_{1}, q_{2}$ on $V, V^{\vee}$ (resp.) and $d \geq 0$ such that

$$
\left|\xi^{\vee}\left(\pi\left(a_{x}\right) \xi\right)\right| \leq q_{1}(\xi) q_{2}\left(\xi^{\vee}\right)(1+|x|)^{d} e^{-\Lambda_{\pi} \cdot x}, \quad \forall x \in \mathbb{R}_{>0}^{k}, \xi \in V, \xi^{\vee} \in V^{\vee} .
$$

For a subset $I=\left\{i_{1}, \ldots, i_{r}\right\}$ of $\{1, \ldots, k\}$ with $i_{1}<\ldots<i_{r}$, let $x_{I}=\left(x_{i_{1}}, \ldots, x_{i_{r}}\right)$ and $\bar{I}=\{1, \ldots, k\}-I$.

Theorem 1.1. Let $\pi \in \operatorname{Alg} G_{k}$ be an irreducible representation on a space $V$. For any $D=$ $\left(D_{1}, \ldots, D_{k}\right) \in \mathbb{R}^{k}$ there exist finite subsets $C_{\ell} \subset E^{(\ell)}(\pi)$ and functions $p_{I, z}: \mathbb{R}^{k} \times V \times V^{\vee} \rightarrow \mathbb{C}$ for $I \subset\{1, \ldots, k\}, z \in \prod_{\ell \in I} C_{\ell}$, such that the following holds.

(1) For all $\xi \in V, \xi^{\vee} \in V^{\vee}$ and $x \in \mathbb{R}^{k}$,

$$
\xi^{\vee}\left(\pi\left(a_{x}\right) \xi\right)=\sum_{I, z} p_{I, z}\left(x ; \xi, \xi^{\vee}\right) \cdot e^{-z \cdot x_{I}},
$$

where the (finite) summation is over all $I \subset\{1, \ldots, k\}$ and $z \in \prod_{\ell \in I} C_{\ell}$.

(2) Each function $p_{I, z}\left(x ; \xi, \xi^{\vee}\right)$ is polynomial in $x_{I}$ :

$$
p_{I, z}\left(x ; \xi, \xi^{\vee}\right)=\sum_{\alpha \in \mathbb{N}^{I}} c_{\alpha}\left(x_{\bar{I}} ; \xi, \xi^{\vee}\right) x_{I}^{\alpha},
$$

where the coefficients $c_{\alpha}\left(x_{\bar{I}} ; \xi, \xi^{\vee}\right)$ are smooth in $x_{\bar{I}}$, linear in $\xi$ and $\xi^{\vee}$, and satisfy bounds of the form

$$
\left|c_{\alpha}\left(x_{\bar{I}} ; \xi, \xi^{\vee}\right)\right| \leq q_{1}(\xi) q_{2}\left(\xi^{\vee}\right)\left(1+\left|x_{\bar{I}}\right|\right)^{d} e^{-D_{\bar{I}} \cdot x_{\bar{I}}}, \quad \forall x_{\bar{I}} \in \mathbb{R}_{>0}^{\bar{I}}, \xi \in V, \xi^{\vee} \in V^{\vee},
$$

with $d \geq 0$ and continuous seminorms $q_{1}, q_{2}$ on $V, V^{\vee}$ (resp.).

Proof. It is enough to show the above statement for arbitrarily large $D_{\ell}, \ell=1, \ldots, k$. We achieve this by first treating the case $D=\Lambda_{\pi}$, then proving that any $D$ can be replaced by $D+e_{\ell}$ for some $\ell=1, \ldots, k$. Repeatedly applying this argument yields the claim.

For $D=\Lambda_{\pi}$ one may choose the sum in (1.5) to consist of only one summand, for the empty set $I$, then the statement follows from (1.4). Now let $D \in \mathbb{R}^{k}$ be arbitrary and assume (1.5), (1.6) and (1.7). We fix $1 \leq \ell \leq k$ and prove that the same is true for $D$ replaced by $D+e_{\ell}$. For brevity, set $H=H_{\ell}, t=x_{\ell}$ and $x^{\prime}=\left(x_{1}, \ldots, x_{\ell-1}, 0, x_{\ell+1}, \ldots, x_{k}\right)$. 
Let $\left\{X_{p}\right\}_{p}$ be a basis of $\mathfrak{u}_{\ell}$. In Wal92, $\S 15.2 .4$ ] Wallach constructed $E_{1}=1, E_{2}, \ldots, E_{r} \in$ $\mathcal{U}\left(\mathfrak{g}_{k}\right)$ as well as finite sets $\left\{Z_{i, j}\right\}_{i, j} \subset \mathcal{Z}\left(\mathfrak{g}_{k}\right)$ and $\left\{U_{p, i}\right\}_{p, i} \subset \mathcal{U}\left(\mathfrak{g}_{k}\right)$ such that

$$
H_{\ell} E_{i}=\sum_{j} Z_{i, j} E_{j}+\sum_{p} X_{p} U_{p, i}, \quad \forall i=1, \ldots, r .
$$

Since $\pi$ is irreducible, the elements $Z_{i, j}$ act by scalars $B_{i, j}=\pi\left(Z_{i, j}\right) \in \mathbb{C}$ and we form the matrix $B=\left(B_{i, j}\right)_{i, j}$. Note that the eigenvalues of $B$ are contained in the finite set $E_{1}^{(\ell)}(\pi)=\left\{\zeta_{1}, \ldots, \zeta_{N}\right\}$ (the generalized eigenvalues of $\pi\left(H_{\ell}\right)$ on the Jacquet module $V / \mathfrak{u}_{\ell} V$ ). Define

$$
\begin{aligned}
F\left(t, x^{\prime} ; \xi, \xi^{\vee}\right) & =\left(\begin{array}{c}
\xi^{\vee}\left(\pi\left(e^{-t H}\right) \pi\left(a_{x^{\prime}}\right) \pi\left(E_{1}\right) \xi\right) \\
\vdots \\
\xi^{\vee}\left(\pi\left(e^{-t H}\right) \pi\left(a_{x^{\prime}}\right) \pi\left(E_{r}\right) \xi\right)
\end{array}\right), \\
G\left(t, x^{\prime} ; \xi, \xi^{\vee}\right) & =\sum_{p}\left(\begin{array}{c}
\xi^{\vee}\left(\pi\left(e^{-t H}\right) \pi\left(a_{x^{\prime}}\right) \pi\left(X_{p}\right) \pi\left(U_{p, 1}\right) \xi\right) \\
\vdots \\
\xi^{\vee}\left(\pi\left(e^{-t H}\right) \pi\left(a_{x^{\prime}}\right) \pi\left(X_{p}\right) \pi\left(U_{p, r}\right) \xi\right)
\end{array}\right)
\end{aligned}
$$

The first coordinate of $F\left(t, x^{\prime} ; \xi, \xi^{\vee}\right)$ is equal to $\xi^{\vee}\left(\pi\left(a_{x}\right) \xi\right)$ and the function $F\left(t, x^{\prime} ; \xi, \xi^{\vee}\right)$ satisfies the following differential equation:

$$
\frac{d}{d t} F\left(t, x^{\prime} ; \xi, \xi^{\vee}\right)=-B F\left(t, x^{\prime} ; \xi, \xi^{\vee}\right)-G\left(t, x^{\prime} ; \xi, \xi^{\vee}\right) .
$$

The solution of this system is given by

$$
F\left(t, x^{\prime} ; \xi, \xi^{\vee}\right)=e^{-t B} F\left(0, x^{\prime} ; \xi, \xi^{\vee}\right)-e^{-t B} \int_{0}^{t} e^{\tau B} G\left(\tau, x^{\prime} ; \xi, \xi^{\vee}\right) d \tau .
$$

We claim that each coordinate on the right-hand side is a finite sum of terms of the form

$$
c\left(x_{\bar{I}} ; \xi, \xi^{\vee}\right) x_{I}^{\alpha} e^{-z \cdot x_{I}}
$$

with $I \subset\{1, \ldots, k\}, \alpha \in \mathbb{N}^{I}$ and $z \in \prod_{i \in I} E^{(i)}(\pi)$, and a function $c\left(x_{\bar{I}} ; \xi, \xi^{\vee}\right)$ which is smooth in $x_{\bar{I}}$, linear in $\xi$ and $\xi^{\vee}$ and satisfies

$$
\left|c\left(x_{\bar{I}} ; \xi, \xi^{\vee}\right)\right| \leq q_{1}(\xi) q_{2}\left(\xi^{\vee}\right)\left(1+\left|x_{\bar{I}}\right|\right)^{d} e^{-\left(D+e_{\ell}\right)_{\bar{I}} \cdot x_{\bar{I}}}, \quad \forall x_{\bar{I}} \in \mathbb{R}_{>0}^{\bar{I}}, \xi \in V, \xi^{\vee} \in V^{\vee},
$$

for some continuous seminorms $q_{1}, q_{2}$ and $d \geq 0$. This will finish the proof.

Note that

$$
e^{\tau B}=\sum_{j=1}^{N} P_{j}(\tau) e^{\zeta_{j} \tau}
$$

for some matrix-valued polynomials $P_{j}(\tau)$. Let us treat the two summands in (1.9) separately. (1) The coordinates of $F\left(0, x^{\prime} ; \xi, \xi^{\vee}\right)$ are of the form $\xi^{\vee}\left(\pi\left(a_{x^{\prime}}\right) \pi\left(E_{i}\right) \xi\right)$, and by (1.12) multiplying with $e^{-t B}$ yields sums of terms of the form

$$
t^{m} e^{-\zeta_{j} t} \cdot \xi^{\vee}\left(\pi\left(a_{x^{\prime}}\right) \pi\left(E_{i}\right) \xi\right),
$$

where $m \geq 0$. The map $\xi \mapsto \pi\left(E_{i}\right) \xi$ is continuous, hence for every continuous seminorm $q_{1}^{\prime}$ on $V$ there exists a continuous seminorm $q_{1}$ such that $q_{1}^{\prime}\left(\pi\left(E_{i}\right) \xi\right) \leq q_{1}(\xi)$ for all 
$\xi \in V$. Applying the induction assumption to the matrix coefficients $\xi^{\vee}\left(\pi\left(a_{x^{\prime}}\right) \pi\left(E_{i}\right) \xi\right)$ then shows that the terms (1.13) are finite sums of the form

$$
t^{m} e^{-\zeta_{j} t} \cdot c\left(x_{\bar{J}}^{\prime} ; \xi, \xi^{\vee}\right)\left(x_{J}^{\prime}\right)^{\alpha} e^{-z^{\prime} \cdot x_{J}^{\prime}}
$$

where $J \subset\{1, \ldots, k\}-\{\ell\}, \bar{J}=\{1, \ldots, k\}-(J \cup\{\ell\}), \alpha \in \mathbb{N}^{J}, z^{\prime} \in \prod_{i \in J} E^{(i)}(\pi)$ and $c\left(x_{\bar{J}}^{\prime} ; \xi, \xi^{\vee}\right)$ is smooth in $x_{\bar{J}}^{\prime}$, linear in $\xi$ and $\xi^{\vee}$, and satisfies

$$
\left|c\left(x_{\bar{J}}^{\prime} ; \xi, \xi^{\vee}\right)\right| \leq q_{1}(\xi) q_{2}\left(\xi^{\vee}\right)\left(1+\left|x_{\bar{J}}^{\prime}\right|\right)^{d} e^{-D_{\bar{J}} \cdot x_{\bar{J}}^{\prime}}, \quad \forall x_{\bar{J}}^{\prime} \in \mathbb{R}_{>0}^{\bar{J}}, \xi \in V, \xi^{\vee} \in V^{\vee},
$$

for some continuous seminorms $q_{1}, q_{2}$ and $d \geq 0$. Let $I=J \cup\{\ell\}, x=x^{\prime}+t e_{\ell}$ and $z=$ $\left(\zeta_{j}, z^{\prime}\right) \in E^{(\ell)}(\pi) \times \prod_{i \in J} E^{(i)}(\pi)$, then (1.14) is clearly of the form (1.10) and $c\left(x_{\bar{I}} ; \xi, \xi^{\vee}\right)$ satisfies (1.11) since $\ell \notin \bar{I}$.

(2) The coordinates of $G\left(t, x^{\prime} ; \xi, \xi^{\vee}\right)$ are sums of the terms

$$
\xi^{\vee}\left(\pi\left(e^{-t H}\right) \pi\left(a_{x^{\prime}}\right) \pi\left(X_{p}\right) \pi\left(U_{p, i}\right) \xi\right)=e^{-t} \xi^{\vee}\left(\pi\left(a_{x^{\prime}}\right) \pi\left(X_{p}\right) \pi\left(e^{-t H}\right) \pi\left(U_{p, i}\right) \xi\right) .
$$

Note that $\pi\left(a_{x^{\prime}}\right) \pi\left(X_{p}\right)$ is a sum of terms of the form $e^{-\beta \cdot x^{\prime}} \pi(X) \pi\left(a_{x^{\prime}}\right)$ with $X \in \mathfrak{u}_{\ell}$ and $\beta \in \mathbb{N}^{k-1}$. Hence, each coordinate of $G\left(t, x^{\prime} ; \xi, \xi^{\vee}\right)$ is a sum of terms of the form

$$
e^{-t} e^{-\beta \cdot x^{\prime}} \cdot\left(\pi^{\vee}(X) \xi^{\vee}\right)\left(\pi\left(e^{-t H}\right) \pi\left(a_{x^{\prime}}\right) \pi(U) \xi\right), \quad U, X \in \mathcal{U}\left(\mathfrak{g}_{k}\right), \beta \in \mathbb{N}^{k-1} .
$$

Since $\xi \mapsto \pi(U) \xi$ and $\xi^{\vee} \mapsto \pi^{\vee}(X) \xi^{\vee}$ are continuous linear operators we can apply the induction assumption to the matrix coefficients $\left(\pi^{\vee}(X) \xi^{\vee}\right)\left(\pi\left(e^{-t H}\right) \pi\left(a_{x^{\prime}}\right) \pi(U) \xi\right)$ and find that the coordinates of $G\left(t, x^{\prime} ; \xi, \xi^{\vee}\right)$ are linear combinations of terms of the form

$$
c\left(x_{\bar{J}}^{\prime} ; \xi, \xi^{\vee}\right)\left(x_{J}^{\prime}\right)^{\alpha} e^{-z^{\prime} \cdot x_{J}^{\prime}} \cdot t^{m} e^{-\left(z_{\ell}+1\right) \cdot t} \quad \text { and } \quad c\left(t, x_{\bar{J}}^{\prime} ; \xi, \xi^{\vee}\right)\left(x_{J}^{\prime}\right)^{\alpha} e^{-z^{\prime} \cdot x_{J}^{\prime}} \cdot e^{-t}
$$

with $J \subset\{1, \ldots, k\}-\{\ell\}, \bar{J}=\{1, \ldots, k\}-(J \cup\{\ell\}), \alpha \in \mathbb{N}^{J}, m \in \mathbb{N}, z^{\prime} \in \prod_{i \in J} E^{(i)}(\pi)$, $z_{\ell} \in E^{(\ell)}(\pi)$, where the coefficients $c\left(x_{\bar{J}}^{\prime} ; \xi, \xi^{\vee}\right)$ and $c\left(t, x_{\bar{J}}^{\prime} ; \xi, \xi^{\vee}\right)$ are smooth in $x_{\bar{J}}^{\prime}$ and also $t$ in the second case, linear in $\xi$ and $\xi^{\vee}$, and satisfy estimates of the form

$$
\begin{aligned}
\left|c\left(x_{\bar{J}}^{\prime} ; \xi, \xi^{\vee}\right)\right| & \leq q_{1}(\xi) q_{2}\left(\xi^{\vee}\right)\left(1+\left|x_{\bar{J}}^{\prime}\right|\right)^{d} e^{-D_{\bar{J}} \cdot x_{\bar{J}}^{\prime}}, \\
\left|c\left(t, x_{\bar{J}}^{\prime} ; \xi, \xi^{\vee}\right)\right| & \leq q_{1}(\xi) q_{2}\left(\xi^{\vee}\right)\left(1+\left|x_{\bar{J}}^{\prime}\right|\right)^{d} e^{-D_{\bar{J}} \cdot x_{\bar{J}}^{\prime}}(1+t)^{d} e^{-D_{\ell} t}
\end{aligned}
$$

for all $x_{\bar{J}}^{\prime} \in \mathbb{R}_{>0}^{\bar{J}}, t \geq 0$ and $\xi \in V, \xi^{\vee} \in V^{\vee}$. Here we have attributed the factors $e^{-\beta_{i} x_{i}}$ either to $e^{-z^{\prime} \cdot x_{J}^{\prime}}$ in case $i \in J$, or to $c\left(x_{\bar{J}}^{\prime} ; \xi, \xi^{\vee}\right)$ and $c\left(t, x_{\bar{J}}^{\prime} ; \xi, \xi^{\vee}\right)$ in case $i \notin J$. Then by (1.12) the components of $e^{(\tau-t) B} G\left(\tau, x^{\prime} ; \xi, \xi^{\vee}\right)$ are linear combinations of terms of the form

$$
\begin{aligned}
& I_{1}=t^{n} e^{-\zeta_{j} t} \cdot c\left(x_{\bar{J}}^{\prime} ; \xi, \xi^{\vee}\right)\left(x_{J}^{\prime}\right)^{\alpha} e^{-z^{\prime} \cdot x_{J}^{\prime}} \cdot \tau^{m} e^{\left(\zeta_{j}-z_{\ell}-1\right) \cdot \tau} \quad \text { and } \\
& I_{2}=t^{n} e^{-\zeta_{j} t} \cdot c\left(\tau, x_{\bar{J}}^{\prime} ; \xi, \xi^{\vee}\right)\left(x_{J}^{\prime}\right)^{\alpha} e^{-z^{\prime} \cdot x_{J}^{\prime}} \cdot \tau^{m} e^{\left(\zeta_{j}-1\right) \tau} .
\end{aligned}
$$

We treat the integration over $\tau$ for these two types separately.

For $I_{1}$ we know that

$$
\int_{0}^{t} \tau^{m} e^{\left(\zeta_{j}-z_{\ell}-1\right) \cdot \tau} d \tau=p(t) e^{\left(\zeta_{j}-z_{\ell}-1\right) t}+C
$$

for some polynomial $p(t)$ and a constant $C$. Hence

$$
\int_{0}^{t} I_{1} d \tau=p(t) t^{n} e^{-\left(z_{\ell}+1\right) t} c\left(x_{\bar{J}}^{\prime} ; \xi, \xi^{\vee}\right)\left(x_{J}^{\prime}\right)^{\alpha} e^{-z^{\prime} \cdot x_{J}^{\prime}}
$$




$$
+C t^{n} e^{-\zeta_{j} t} c\left(x_{\bar{J}}^{\prime} ; \xi, \xi^{\vee}\right)\left(x_{J}^{\prime}\right)^{\alpha} e^{-z^{\prime} \cdot x_{J}^{\prime}} .
$$

Both summands are linear combinations of terms of the form (1.10) for $I=J \cup\{\ell\}$ since both $z_{\ell}+1, \zeta_{j} \in E^{(\ell)}(\pi)$, and the corresponding estimates (1.11) follow from (1.15) since $\ell \notin \bar{I}$.

For $I_{2}$ we first assume $D_{\ell}>\operatorname{Re} \zeta_{j}-1$. Then rewrite

$$
\int_{0}^{t} I_{2} d \tau=\int_{0}^{\infty} I_{2} d \tau-\int_{t}^{\infty} I_{2} d \tau
$$

The first integral

$$
\int_{0}^{\infty} I_{2} d \tau=t^{n} e^{-\zeta_{j} t}\left(x_{J}^{\prime}\right)^{\alpha} e^{-z^{\prime} \cdot x_{J}^{\prime}} \int_{0}^{\infty} c\left(\tau, x_{\bar{J}}^{\prime} ; \xi, \xi^{\vee}\right) \tau^{m} e^{\left(\zeta_{j}-1\right) \tau} d \tau
$$

is of the form (1.10) for $I=J \cup\{\ell\}$. The estimate (1.11) follows from (1.16) since $\left|c\left(\tau, x_{\bar{J}}^{\prime} ; \xi, \xi^{\vee}\right) \tau^{m} e^{\left(\zeta_{j}-1\right) \tau}\right|$ is bounded by

$$
q_{1}(\xi) q_{2}\left(\xi^{\vee}\right)\left(1+\left|x_{\bar{J}}^{\prime}\right|\right)^{d} e^{-D_{\bar{J}} \cdot x_{\bar{J}}^{\prime}} \cdot \tau^{m}(1+\tau)^{d} e^{\left(\operatorname{Re} \zeta_{j}-D_{\ell}-1\right) \tau},
$$

which is integrable over $\mathbb{R}_{>0}$ since $D_{\ell}>\operatorname{Re} \zeta_{j}-1$.

The second integral

$$
\int_{t}^{\infty} I_{2} d \tau=\left(x_{J}^{\prime}\right)^{\alpha} e^{-z^{\prime} \cdot x_{J}^{\prime}} \cdot t^{n} e^{-\zeta_{j} t} \int_{t}^{\infty} c\left(\tau, x_{\bar{J}}^{\prime} ; \xi, \xi^{\vee}\right) \tau^{m} e^{\left(\zeta_{j}-1\right) \tau} d \tau
$$

is of the form (1.10) for $I=J$. The integral is by the previous estimation smooth in $t$ and $x_{\bar{J}}^{\prime}$, and the estimate (1.11) follows from

$$
\begin{aligned}
& \left|t^{n} e^{-\zeta_{j} t} \int_{t}^{\infty} c\left(\tau, x_{\bar{J}}^{\prime} ; \xi, \xi^{\vee}\right) \tau^{m} e^{\left(\zeta_{j}-1\right) \tau} d \tau\right| \\
& \leq q_{1}(\xi) q_{2}\left(\xi^{\vee}\right)\left(1+\left|x_{\bar{J}}^{\prime}\right|\right)^{d} e^{-D_{\bar{J}} \cdot x_{\bar{J}}^{\prime}} \cdot t^{n} e^{-\operatorname{Re} \zeta_{j} t} \int_{t}^{\infty} \tau^{m}(1+\tau)^{d} e^{\left(\operatorname{Re} \zeta_{j}-D_{\ell}-1\right) \tau} d \tau \\
& \leq \text { constant } \times q_{1}(\xi) q_{2}\left(\xi^{\vee}\right)\left(1+\left|x_{\bar{J}}^{\prime}\right|\right)^{d} e^{-D_{\bar{J}} \cdot x_{\bar{J}}^{\prime}} \cdot(1+t)^{m+d+n} e^{-\left(D_{\ell}+1\right) t} .
\end{aligned}
$$

It remains to consider $I_{2}$ when $D_{\ell} \leq \operatorname{Re} \zeta_{j}-1$. Then

$$
\int_{0}^{t} I_{2} d \tau=\left(x_{J}^{\prime}\right)^{\alpha} e^{-z^{\prime} \cdot x_{J}^{\prime}} \cdot t^{n} e^{-\zeta_{j} t} \int_{0}^{t} c\left(\tau, x_{\bar{J}}^{\prime} ; \xi, \xi^{\vee}\right) \tau^{m} e^{\left(\zeta_{j}-1\right) \tau} d \tau
$$

is of the form (1.10) for $I=J$. The estimate (1.11) now follows from (1.16) since

$$
\begin{aligned}
& \left|t^{n} e^{-\zeta_{j} t} \int_{0}^{t} c\left(\tau, x_{\bar{J}}^{\prime} ; \xi, \xi^{\vee}\right) \tau^{m} e^{\left(\zeta_{j}-1\right) \tau} d \tau\right| \\
& \leq q_{1}(\xi) q_{2}\left(\xi^{\vee}\right)\left(1+\left|x_{\bar{J}}^{\prime}\right|\right)^{d} e^{-D_{\bar{J}} \cdot x_{\bar{J}}^{\prime}} \cdot t^{n} e^{-\left(\operatorname{Re} \zeta_{j}\right) t} \int_{0}^{t} \tau^{m}(1+\tau)^{d} e^{\left(\operatorname{Re} \zeta_{j}-D_{\ell}-1\right) \tau} d \tau \\
& \leq q_{1}(\xi) q_{2}\left(\xi^{\vee}\right)\left(1+\left|x_{\bar{J}}^{\prime}\right|\right)^{d} e^{-D D_{\bar{J}} \cdot x_{\bar{J}}^{\prime}} \cdot t^{n+1}(1+t)^{d} e^{-\left(D_{\ell}+1\right) t} .
\end{aligned}
$$

The proof is complete. 
1.6. The Weil symbol. Let $F$ be a local field. We usually denote by $\psi$ a nontrivial additive character of $F$. Then $\gamma_{\psi}$ is the normalized Weil factor $([$ Wei64, $\S 14]), \gamma_{\psi}(\cdot)^{4}=1$. For $a \in F^{*}, \psi_{a}$ denotes the character $\psi_{a}(x)=\psi(a x)$ and $\gamma_{\psi, a}=\gamma_{\psi_{a}}$. We have the following formulas (see the appendix of [Rao93], $\gamma_{\psi}(a)$ is $\gamma_{F}(a, \psi)$ in his notation):

$$
\gamma_{\psi}(x y)=\gamma_{\psi}(x) \gamma_{\psi}(y)(x, y)_{2}, \quad \gamma_{\psi}\left(x^{2}\right)=1, \quad \gamma_{\psi}^{-1}=\gamma_{\psi^{-1}}, \quad \gamma_{\psi, a}(x)=(a, x)_{2} \gamma_{\psi}(x)
$$

\section{Metaplectic Hironaka Theory}

2.1. Preliminaries. In this section $F$ is a local non-Archimedean field and $G_{n}=G_{n}(F)$. Then $\mathcal{O}, \varpi, K$ and $q$ are defined as in $\S$ 1.3. Assume $q>3$ and $|2|=1$ in the field. We normalize the Haar measure $d g$ on $G_{n}$ such that $\operatorname{vol}(K)=1$. Let $\kappa: K \rightarrow \widetilde{G}_{n}$ be the canonical splitting of $K$. It coincides with $\mathfrak{s}$ on $T_{n} \cap K, \mathfrak{W}$ and $N_{n} \cap K$ (W was defined in $\S[1.2$, and is a subset of $K)$. We use this freely, e.g., it implies that $\mathfrak{s}$ is a splitting of $\mathfrak{W}$.

2.2. Metaplectic review of Casselman's theory. In this section we describe a metaplectic analog of several results of Casselman [Cas80], needed in the following section to prove a result of Hironaka [Hir99]. We define an analog of Casselman's basis for the Iwahori fixed subspace of an unramified principal series. Following [Hir99], we use this basis to deduce a general formula for functionals on this space. The bulk part of this section has already appeared in the works of Chinta and Offen [CO13] and McNamara [McN12], specific references are given below.

In order to apply the ideas of Cas80] we need several auxiliary results. Let $\mathcal{I}<K$ be the Iwahori subgroup which is compatible with $B_{n}$. The section $\kappa$ is in particular a splitting of $\mathcal{I}$. The Iwahori factorization of $\mathcal{I}$ reads $\mathcal{I}=\mathcal{I}^{+} \mathcal{I}^{0} \mathcal{I}^{-}$where $\mathcal{I}^{+}=\mathcal{I} \cap N_{n}, \mathcal{I}^{0}=\mathcal{I} \cap T_{n}$ and $\mathcal{I}^{-}=\mathcal{I} \cap N_{n}^{-}$. Note that

$$
\mathcal{I}=\left(\mathcal{I} \cap B_{n}\right) \mathcal{I}^{-}=\left(K \cap B_{n}\right) \mathcal{I}^{-} .
$$

For a representation $\pi \in \operatorname{Alg} \widetilde{G}_{n}$ and a compact open subgroup $K_{0}<K$, the space of $\kappa\left(K_{0}\right)$-invariants is denoted $\pi^{K_{0}}$ and the projection on $\pi^{K_{0}}$ is denoted $\mathscr{P}_{K_{0}}$. For any vector $\xi$ in the space of $\pi$,

$$
\mathscr{P}_{K_{0}}(\xi)=\int_{K_{0}} \pi(\kappa(k)) \xi d k
$$

where the measure is normalized such that $\operatorname{vol}\left(K_{0}\right)=1$.

Let

$$
T_{n}^{-}=\left\{t \in T_{n}:|\alpha(t)| \leq 1, \quad \forall \alpha \in \Delta_{G_{n}}\right\} .
$$

The following is a version of Jacquet's First Lemma.

Claim 2.1. Let $\pi \in \operatorname{Alg} \widetilde{G}_{n}$ be admissible. For any $\xi$ in the space of $\pi^{\mathcal{I}^{0} \mathcal{I}^{-}}$and $t \in C_{\widetilde{T}_{n}} \cap \widetilde{T}_{n}^{-}$,

$$
\mathscr{P}_{\mathcal{I}}(\pi(t) \xi)=\mathscr{P}_{\mathcal{I}^{+}}(\pi(t) \xi), \quad \mathscr{P}_{\mathcal{I}}(\pi(t) \xi)-\pi(t) \xi \in \pi\left(N_{n}\right) .
$$

Proof. Write the integral $\mathscr{P}_{\mathcal{I}}$ using the Iwahori decomposition $\mathcal{I}=\mathcal{I}^{+} \mathcal{I}^{0} \mathcal{I}^{-}$. Then

$$
\begin{aligned}
\mathscr{P}_{\mathcal{I}}(\pi(t) \xi) & =\int_{\mathcal{I}^{+}} \int_{\mathcal{I}^{0}} \int_{\mathcal{I}^{-}} \pi(\kappa(x) \kappa(y) \kappa(z) t) \xi d z d y d x \\
& =\int_{\mathcal{I}^{+}} \int_{\mathcal{I}^{0}} \int_{\mathcal{I}^{-}} \pi\left(\kappa(x) t \kappa(y){ }^{t^{-1}} \kappa(z)\right) \xi d z d y d x
\end{aligned}
$$




$$
=\mathscr{P}_{\mathcal{I}^{+}}(\pi(t) \xi) .
$$

Here we used the facts that ${ }^{t^{-1}} \kappa\left(\mathcal{I}^{-}\right)<\kappa\left(\mathcal{I}^{-}\right), t$ commutes with $\kappa\left(\mathcal{I}^{0}\right)$ and $\xi \in \pi^{\mathcal{I}^{0} \mathcal{I}^{-}}$. Then the Jacquet-Langlands lemma (see e.g., [BZ76, 2.33]) implies that $\mathscr{P}_{\mathcal{I}^{+}}(\pi(t) \xi)$ and $\pi(t) \xi$ have the same image in $\pi_{N_{n}}$.

The following claim is a weak analog of [Cas80, Proposition 2.4].

Claim 2.2. Let $\pi \in \operatorname{Alg} \widetilde{G}_{n}$ be admissible. The representation $\pi^{\mathcal{I}}$ injects as a vector space into $\pi_{N_{n}}^{\mathcal{I}_{0}}$.

Proof. We have to prove $\pi^{\mathcal{I}} \cap \pi\left(N_{n}\right)=0$. Let $\xi$ belong to the space of $\pi^{\mathcal{I}} \cap \pi\left(N_{n}\right)$. We prove $\xi=0$. According to the Jacquet-Langlands lemma ([BZ76, 2.33]) there is a compact open subgroup $\mathcal{N}<N_{n}$ with

$$
\int_{\mathcal{N}} \pi(v) \xi d v=0
$$

Let $t \in C_{\widetilde{T}_{n}} \cap \widetilde{T}_{n}^{-}$be such that $\mathcal{N}<{ }^{p(t)^{-1}} \mathcal{I}^{+}$. Then by Claim 2.1,

$$
\mathscr{P}_{\mathcal{I}}(\pi(t) \xi)=\int_{\mathcal{I}^{+}} \pi(\kappa(x) t) \xi d x=\pi(t) \int_{\mathcal{I}^{+}} \pi\left(\mathfrak{s}\left({ }^{p(t)^{-1}} x\right)\right) \xi d x=0,
$$

by our choice of $t$. However, by [Sav88, Proposition 3.1.4] the mapping $\xi \mapsto \mathscr{P}_{\mathcal{I}}(\pi(t) \xi)$ on the finite-dimensional space $\pi^{\mathcal{I}}$ is invertible (this mapping is essentially $\pi\left(T_{p(t)}\right)$ in the notation of loc. cit.).

Remark 2.3. The original claim in [Cas80] also included surjectivity. In the metaplectic case this does not hold in general, e.g., when $\pi$ is a genuine unramified principal series.

We recall the parametrization of genuine unramified principal series representations of $\widetilde{G}_{n}$. Let $\widetilde{T}_{n, *}$ be the centralizer of $\widetilde{T}_{n} \cap \kappa(K)$ in $\widetilde{T}_{n}$, it is a maximal abelian subgroup. Explicitly,

$$
T_{n, *}=\left\{\operatorname{diag}\left(t_{1} z, \ldots, t_{n} z\right): t_{i} \in F^{* 2} \mathcal{O}^{*}, z \in F^{* e}\right\}
$$

(e was defined in $\S$ 1.2). Assume $\underline{s} \in \mathbb{C}^{n}$. Let $\gamma: F^{*} \rightarrow \mathbb{C}$ be a function satisfying $\gamma\left(z z^{\prime}\right)=\gamma(z) \gamma\left(z^{\prime}\right) \sigma\left(z I_{n}, z^{\prime} I_{n}\right)$ for $z, z^{\prime} \in F^{* \mathrm{e}}$, and $\left.\gamma\right|_{F^{* 2}}=\left.\gamma\right|_{\mathcal{O}^{*}}=1$. The genuine unramified character $\chi=\chi_{\underline{s}, \gamma}$ of $\widetilde{T}_{n, *}$ is defined by

$$
\chi\left(\epsilon \mathfrak{s}\left(\operatorname{diag}\left(t_{1}, \ldots, t_{n}\right)\right) \mathfrak{s}\left(z I_{n}\right)\right)=\epsilon \gamma(z)|z|^{s_{1}+\ldots+s_{n}} \prod_{i=1}^{n}\left|t_{i}\right|^{s_{i}}, \quad \forall \epsilon \in \mu_{2}, t_{i} \in F^{* 2} \mathcal{O}^{*}, z \in F^{* \mathrm{e}} .
$$

Put $B_{n, *}=T_{n, *} N_{n}$, it is a closed subgroup of $G_{n}$, open and normal in $B_{n}$. Then

$$
\mathrm{I}(\chi)=\operatorname{Ind}_{\widetilde{B}_{n, *}}^{\widetilde{G}_{n}}\left(\delta_{B_{n}}^{1 / 2} \chi\right)
$$

is a genuine unramified principal series representation, i.e., $\mathrm{I}(\chi)^{K}$ is one dimensional, and any such representation takes this form, where $s_{i}$ is unique modulo $\frac{\pi i}{\log q} \mathbb{Z}$ (as opposed to $\frac{2 \pi i}{\log q} \mathbb{Z}$ in $G_{n}$ ). The dependency on $\gamma$ will not appear in the formulas and in fact when $n$ is even, $\gamma$ is irrelevant. For a general discussion of these representation for $r$-fold covers of $G_{n}$ see CO13.

We regard the elements in the space of $\mathrm{I}(\chi)$ as complex-valued functions. The unramified normalized element of $\mathrm{I}(\chi)$ is the unique function $f_{\chi} \in \mathrm{I}(\chi)$ such that $f_{\chi}(g \kappa(k))=f_{\chi}(g)$ 
for any $g \in \widetilde{G}_{n}$ and $k \in K$, and $f_{\chi}\left(\mathfrak{s}\left(I_{n}\right)\right)=1$. We will use the observation of [KP84] (Lemma I.1.3, see also McN12] Lemma 2) that for any unramified $f_{\chi}$,

$$
f_{\chi}(\mathfrak{s}(t))=0, \quad \forall t \in T_{n}-T_{n, *} .
$$

Let $\mathcal{S}^{\text {gen }}\left(\widetilde{G}_{n}\right)$ denote the subspace of $\mathcal{S}\left(\widetilde{G}_{n}\right)$ consisting of genuine functions. Given an open subset $K_{0} \subset K$, one can lift any $f \in \mathcal{S}\left(K_{0}\right)$ to $f^{\text {gen }} \in \mathcal{S}^{\text {gen }}\left(\widetilde{G}_{n}\right)$ by putting

$$
f^{\text {gen }}(g)= \begin{cases}\epsilon f\left(k_{0}\right) & g=\epsilon \kappa\left(k_{0}\right), \epsilon \in \mu_{2}, k_{0} \in K_{0}, \\ 0 & g \notin \widetilde{K}_{0} .\end{cases}
$$

For example, for any subset $X \subset G_{n}$ let $\operatorname{ch}_{X}$ be the characteristic function of $X$, then $\operatorname{ch}_{K}^{\text {gen }}$ vanishes off $\widetilde{K}$ and is 1 on $\kappa(K)$.

The group $\widetilde{G}_{n}$ acts on the right on $\mathcal{S}^{\text {gen }}\left(\widetilde{G}_{n}\right)$. We have a surjection $P_{\chi}: \mathcal{S}^{\text {gen }}\left(\widetilde{G}_{n}\right) \rightarrow \mathrm{I}(\chi)$ of $\widetilde{G}_{n}$-representations

$$
\begin{aligned}
P_{\chi}(f)(g) & =\int_{B_{n, *}} f\left(\mathfrak{s}\left(b_{*}\right) g\right) \delta_{B_{n}}^{1 / 2}\left(b_{*}\right) \chi^{-1}\left(\mathfrak{s}\left(b_{*}\right)\right) d_{l} b_{*} \\
& =\int_{T_{n, *}} \int_{N_{n}} f(\mathfrak{s}(t n) g) \delta_{B_{n}}^{1 / 2}(t) \chi^{-1}(\mathfrak{s}(t)) d n d t .
\end{aligned}
$$

Here $d_{l} b_{*}$ is the left Haar measure normalized by requiring $\operatorname{vol}\left(B_{n, *} \cap K\right)=1$, this is consistent with the normalization in the non-metaplectic setting because $B_{n, *} \cap K=B_{n} \cap K$. Note that $\mathfrak{s}$ is a splitting of $B_{n, *}$.

The unramified normalized function of $\mathrm{I}(\chi)$ is then $\varphi_{K, \chi}=P_{\chi}\left(\operatorname{ch}_{K}^{\mathrm{gen}}\right)$.

For $w \in W$, let $\mathfrak{w} \in \mathfrak{W}$ be a representative of $w$ and define $\varphi_{w, \chi}=P_{\chi}\left(\operatorname{ch}_{\mathcal{I} \mathfrak{w} \mathcal{I}}^{\text {gen }}\right)$. This is the function supported on $\widetilde{B}_{n, *} \kappa(\mathfrak{w} \mathcal{I})=\widetilde{B}_{n, *} \mathfrak{s}(\mathfrak{w}) \kappa(\mathcal{I})$, which is right-invariant by $\kappa(\mathcal{I})$ and $\varphi_{w, \chi}(\mathfrak{s}(\mathfrak{w}))=1$. Then $\left\{\varphi_{w, \chi}\right\}_{w \in W}$ is a basis of $\mathrm{I}(\chi)^{\mathcal{I}}$. In particular

$$
\varphi_{K, \chi}=\sum_{w} \varphi_{w, \chi}
$$

We introduce the intertwining operators. If $\alpha \in \Sigma_{G_{n}}$, let $N_{\alpha}<G_{n}$ denote the root subgroup of $\alpha$. For any $w \in W$, let

$$
T_{w}=T_{w, \chi}: \mathrm{I}(\chi) \rightarrow \mathrm{I}\left({ }^{w} \chi\right)
$$

be the standard intertwining operator defined by

$$
T_{w} f_{\chi}(g)=\int_{N_{n}(w)} f_{\chi}\left(\mathfrak{s}\left(\mathfrak{w}^{-1} v\right) g\right) d v,
$$

where $\mathfrak{w} \in \mathfrak{W}$ is the representative of $w$ and

$$
N_{n}(w)=\prod_{\left\{\alpha>0: w^{-1} \alpha<0\right\}} N_{\alpha}<N_{n} .
$$

Given that $\chi$ lies in a certain cone, the integral is absolutely convergent, otherwise it is defined by meromorphic continuation in $\chi$ (i.e., in the parameter $\underline{s}$ ).

Henceforth assume $\chi$ is regular, i.e., ${ }^{w} \chi \neq \chi$ for any $e \neq w \in W$. In this case (see [McN12, Theorem 4])

$$
\operatorname{dim} \operatorname{Hom}_{\widetilde{G}_{n}}\left(\mathrm{I}(\chi), \mathrm{I}\left({ }^{w} \chi\right)\right)=1, \quad \forall w \in W
$$


We recall the Gindikin-Karpelevich formula (Cas80] Theorem 3.1), whose extension to $\widetilde{G}_{n}$ was proved in KP84. For any root $\alpha$, let $\mathcal{R}_{\alpha}$ be the group generated by $N_{\alpha}$ and $N_{-\alpha}$ $\left(\mathcal{R}_{\alpha} \cong \mathrm{SL}_{2}\right), a_{\alpha}^{\prime}$ be the embedding of $\operatorname{diag}\left(\varpi^{2}, \varpi^{-2}\right)$ in $\mathcal{R}_{\alpha}$ and put $a_{\alpha}=\mathfrak{s}\left(a_{\alpha}^{\prime}\right)$. Define for $\alpha$ and $w \in W$,

$$
c_{\alpha}(\chi)=\frac{1-q^{-1} \chi\left(a_{\alpha}\right)}{1-\chi\left(a_{\alpha}\right)}, \quad c_{w}(\chi)=\prod_{\alpha>0: w \alpha<0} c_{\alpha}(\chi) .
$$

Then

$$
T_{w} \varphi_{K, \chi}=c_{w}(\chi) \varphi_{K,{ }^{w} \chi}
$$

This implies the analog of [Cas95, Proposition 3.5]:

Claim 2.4. $T_{w}\left(=T_{w, \chi}\right)$ is an isomorphism if and only if $c_{w^{-1}}\left({ }^{w} \chi\right) c_{w}(\chi) \neq 0$. Furthermore, $\mathrm{I}(\chi)$ is irreducible if and only if $T_{w_{0}}$ is an isomorphism.

Proof. The first part follows exactly as in Cas95. For the second part one can verify that the arguments in the case of $G_{n}$ apply here as well (see Cas95] Theorem 6.6.2). Briefly, if $T_{w_{0}}$ is not an isomorphism, either $I(\chi)$ or $I\left({ }^{w_{0}} \chi\right)$ is reducible, but these representations have the same irreducible constituents ([BZ77, Theorem 2.9]). Suppose $T_{w_{0}}$ is an isomorphism and $\pi$ is a proper irreducible quotient of $\mathrm{I}(\chi)$. Then $\pi$ can be embedded in $\mathrm{I}\left({ }^{w} \chi\right)$ for some $w \in W$ ([BZ77, Theorem 2.9 and Theorem 2.4b]). This embedding is proportional to $T_{w}$ by (2.5), and it follows that $T_{w}$ is not an isomorphism, thus $c_{w}(\chi)$ or $c_{w^{-1}}\left({ }^{w} \chi\right)$ vanish. But then $c_{w_{0}}(\chi)$ or $c_{w_{0}}\left(\chi^{-1}\right)$ is zero, contradiction.

We mention that when $\ell(w)+\ell\left(w^{\prime}\right)=\ell\left(w w^{\prime}\right), T_{w^{\prime},{ }^{w} \chi} T_{w, \chi}=T_{w^{\prime} w, \chi}$, and in general for any $w, w^{\prime}$ (see e.g., [CO13, (3.17)]),

$$
T_{w^{\prime},{ }^{w} \chi} T_{w, \chi}=\frac{c_{w^{\prime}}\left({ }^{w} \chi\right) c_{w}(\chi)}{c_{w^{\prime} w}(\chi)} T_{w^{\prime} w, \chi}
$$

In contrast with the non-metaplectic case, here $\mathrm{I}(\chi)_{N_{n}}$ is not linearly isomorphic with $\mathrm{I}(\chi)^{\mathcal{I}}$ (compare to [Cas80, Proposition 2.4]). Indeed, the dimension of the former is $|W| \cdot\left|T_{n, *} \backslash T_{n}\right|$. However, we can still define an analog of the Casselman basis.

For each $w \in W$ let $\Upsilon_{w}$ be the functional on $\mathrm{I}(\chi)$ given by

$$
\Upsilon_{w}\left(f_{\chi}\right)=T_{w} f_{\chi}\left(\mathfrak{s}\left(I_{n}\right)\right) .
$$

Clearly

$$
\Upsilon_{w}\left(t f_{\chi}\right)=\delta_{B_{n}}^{1 / 2}{ }^{w} \chi(t) \Upsilon_{w}\left(f_{\chi}\right), \quad \forall f_{\chi} \in \mathrm{I}(\chi), \quad t \in C_{\widetilde{T}_{n}}
$$

For any genuine character $\omega$ of $\widetilde{T}_{n, *}$, let $\rho(\omega)=\operatorname{ind}_{\widetilde{T}_{n, *}}^{\widetilde{T}_{n}}(\omega)$. In fact $\rho(\omega)$ depends only on the restriction of $\omega$ to $C_{\widetilde{T}_{n}}$. According to the Geometric Lemma of Bernstein and Zelevinsky [BZ77, Theorem 5.2], and because $\chi$ is regular,

$$
\mathrm{I}(\chi)_{N_{n}}=\bigoplus_{w \in W} \delta_{B_{n}}^{1 / 2} \rho\left({ }^{w} \chi\right)
$$

Since $\Upsilon_{w}$ factors through $\mathrm{I}(\chi)_{N_{n}}$, we can consider it as a functional on this finite direct sum, of finite-dimensional vector spaces. For any $w^{\prime} \neq w$, if $f_{\chi}+\mathrm{I}(\chi)\left(N_{n}\right)$ belongs to the space of $\rho\left({ }^{w^{\prime}} \chi\right)$

$$
\Upsilon_{w}\left(t f_{\chi}\right)=\Upsilon_{w}\left(\delta_{B_{n}}^{1 / 2} w^{\prime} \chi(t) f_{\chi}\right)=\delta_{B_{n}}^{1 / 2}{ }^{w^{\prime}} \chi(t) \Upsilon_{w^{\prime}}\left(f_{\chi}\right), \quad \forall t \in C_{\widetilde{T}_{n}}
$$


Combining this with (2.8) implies that $\Upsilon_{w}$ vanishes on $\bigoplus_{w^{\prime} \neq w} \delta_{B_{n}}^{1 / 2} \rho\left({ }^{w^{\prime}} \chi\right)$.

Claim 2.5. The functionals $\left\{\Upsilon_{w}\right\}_{w}$ restricted to $\mathrm{I}(\chi)^{\mathcal{I}}$ are linearly independent.

Proof. According to [CO13, Remark 1], if we put

$$
\begin{gathered}
f_{w, \chi}=T_{w^{-1} w_{0}} \varphi_{w_{0},{ }^{\left(w^{-1} w_{0}\right)^{-1} \chi}} \in \mathrm{I}(\chi)^{\mathcal{I}}, \\
\Upsilon_{w^{\prime}}\left(f_{w, \chi}\right)=\delta_{w^{\prime}, w}
\end{gathered}
$$

where $\delta_{w^{\prime}, w}$ is the Kronecker delta.

Let $\left\{f_{w, \chi}\right\}_{w}$ be the basis of $\mathrm{I}(\chi)^{\mathcal{I}}$ dual to $\left\{\Upsilon_{w}\right\}_{w}$, given in the proof of Claim 2.5. By definition

$$
f_{w_{0}, \chi}=\varphi_{w_{0}, \chi}
$$

as in the non-metaplectic setting ([Cas95, Proposition 3.7]) the computation there can also be performed here, to obtain a direct proof).

Next we compute the image of $f_{w, \chi}$ in $\mathrm{I}(\chi)_{N_{n}}$ and relate it to the direct sum of (2.9).

Claim 2.6. The image of $f_{w, \chi}$ in $\mathrm{I}(\chi)_{N_{n}}$ belongs to the space of $\rho\left({ }^{w} \chi\right)$. Consequently, for any $t \in C_{\widetilde{T}_{n}}$, the image of $t f_{w, \chi}$ in $\mathrm{I}(\chi)_{N_{n}}$ is $\delta_{B_{n}}^{1 / 2}{ }^{w} \chi(t) f_{w, \chi}+\mathrm{I}(\chi)\left(N_{n}\right)$.

Proof. Looking at (2.9), we can write the image of $f_{w, \chi}$ in $\mathrm{I}(\chi)_{N_{n}}$ in the form $\sum_{w \in W} \xi_{w}$, $\xi_{w} \in \rho\left({ }^{w} \chi\right)$. Fix $w^{\prime} \neq w$. We prove $\xi_{w^{\prime}}=0$, by showing that any functional on $\rho\left({ }^{w^{\prime}} \chi\right)$ vanishes on $\xi_{w^{\prime}}$.

For a representative $b$ of $T_{n, *} \backslash T_{n}$, consider the linear functional on $\mathrm{I}(\chi)$ given by

$$
\Upsilon_{w^{\prime}, b}\left(f_{\chi}\right)=T_{w^{\prime}} f_{\chi}(\mathfrak{s}(b)) .
$$

It is defined on $\mathrm{I}(\chi)_{N_{n}}$ and satisfies equalities similar to (2.8) and (2.10), i.e.,

$$
\begin{array}{ll}
\Upsilon_{w^{\prime}, b}\left(t f_{\chi}\right)=\delta_{B_{n}}^{1 / 2} w^{\prime} \chi(t) \Upsilon_{w^{\prime}, b}\left(f_{\chi}\right), & \forall t \in C_{\widetilde{T}_{n}}, \\
\Upsilon_{w^{\prime}, b}\left(t f_{\chi}\right)=\delta_{B_{n}}^{1 / 2} w^{\prime \prime} \chi(t) \Upsilon_{w^{\prime}, b}\left(f_{\chi}\right), \quad \forall t \in C_{\widetilde{T}_{n}}, \quad f_{\chi}+\mathrm{I}(\chi)\left(N_{n}\right) \in \rho\left(\text { w }^{\prime \prime} \chi\right) .
\end{array}
$$

Hence $\Upsilon_{w^{\prime}, b}$ vanishes on $\rho\left({ }^{w^{\prime \prime}} \chi\right)$ for any $w^{\prime \prime} \neq w^{\prime}$.

The set $\left\{\Upsilon_{w^{\prime}, b}\right\}_{b}$ is linearly independent, since for $b_{1} \in T_{n}$,

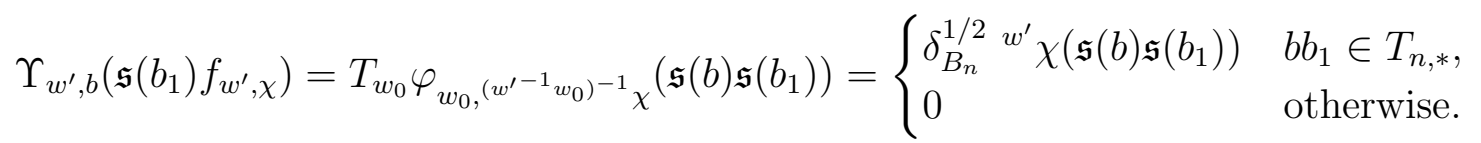

This is because for $t \in T_{n}$, if $\mathfrak{w}_{0} N_{n} t$ intersects the projection of the support of $\varphi_{w_{0}, \ldots .}$, then $t \in T_{n, *}$ (use (2.1) and the fact that ${ }^{\mathfrak{w}_{0}} t \in B_{n, *} N_{n}^{-}$implies $t \in T_{n, *}$ ).

Thus $\left\{\Upsilon_{w^{\prime}, b}\right\}_{b}$ is a basis of the linear dual of $\rho\left(w^{\prime} \chi\right)$ and moreover,

$$
\Upsilon_{w^{\prime}, b}\left(\xi_{w^{\prime}}\right)=\Upsilon_{w^{\prime}, b}\left(f_{w, \chi}\right) \text {. }
$$

Recall that we have to show $\xi_{w^{\prime}}=0$, we prove $\Upsilon_{w^{\prime}, b}\left(\xi_{w^{\prime}}\right)=0$ for all $b \in T_{n}$. For $b \in T_{n, *}$, $\Upsilon_{w^{\prime}, b}$ is a scalar multiple of $\Upsilon_{w^{\prime}}$, hence vanishes on $f_{w, \chi}$ (by definition). It remains to show $\Upsilon_{w^{\prime}, b}\left(f_{w, \chi}\right)=0$ for all $b \notin T_{n, *}$. Using (2.7),

$$
\Upsilon_{w^{\prime}, b}\left(f_{w, \chi}\right)=\frac{c_{w^{\prime}}\left(w^{-1} w_{0} \chi\right) c_{w^{-1} w_{0}}(\chi)}{c_{w^{\prime} w^{-1} w_{0}}(\chi)} T_{w^{\prime} w^{-1} w_{0}} \varphi_{\left.w_{0}, w^{-1} w_{0}\right)^{-1} \chi}(\mathfrak{s}(b))=0,
$$


since for any $\mathfrak{w} \in \mathfrak{W}$ and $v \in N_{n}, \mathfrak{w} v b \in B_{n, *} \mathfrak{w}_{0} \mathcal{I}$ implies $b \in T_{n, *}$ (use (2.1), first deduce $\left.\mathfrak{w}=\mathfrak{w}_{0}\right)$.

Remark 2.7. This claim will be used to prove Claim 2.8 below, which is the analog of Cas80, Proposition 3.9]. In Cas80], the fact that the image of $f_{w, \chi}$ in $\mathrm{I}(\chi)_{N_{n}}$ belongs to $\rho\left({ }^{w} \chi\right)$ followed directly from the definition of the basis $\left\{f_{w, \chi}\right\}$, because in the non-metaplectic setting the spaces ${ }^{w} \rho(\chi)$ are one-dimensional, then $\Upsilon_{w^{\prime}}\left(f_{w, \chi}\right)=0$ immediately implies $\xi_{w^{\prime}}=0$.

Claim 2.8. (Cas80, Proposition 3.9]) For any $w \in W$ and $t \in C_{\widetilde{T}_{n}} \cap \widetilde{T}_{n}^{-}$,

$$
\mathscr{P}_{\mathcal{I}}\left(t f_{w, \chi}\right)=\delta_{B_{n}}^{1 / 2}{ }^{w} \chi(t) f_{w, \chi} .
$$

Proof. By Claims 2.1 and 2.6, $\mathscr{P}_{\mathcal{I}}\left(t f_{w, \chi}\right)$ and $\delta_{B_{n}}^{1 / 2}{ }^{w} \chi(t) f_{w, \chi}$ have the same image in $\mathrm{I}(\chi)_{N_{n}}$. Because $f_{w, \chi}=\mathscr{P}_{\mathcal{I}}\left(f_{w, \chi}\right)$, this implies

$$
\mathscr{P}_{\mathcal{I}}\left(t f_{w, \chi}-\delta_{B_{n}}^{1 / 2}{ }^{w} \chi(t) f_{w, \chi}\right) \in \mathrm{I}(\chi)\left(N_{n}\right) \cap \mathrm{I}(\chi)^{\mathcal{I}}=0,
$$

where we used Claim 2.2. The result follows.

For each $w \in W$, if $\mathfrak{w}$ is the representative of $w$, put $q(w)=[\mathcal{I} \mathfrak{w} \mathcal{I}: \mathcal{I}]$. E.g., $q\left(s_{\alpha}\right)=q$ for $\alpha \in \Delta_{G_{n}}$. Then $[K: \mathcal{I}]=\sum_{w \in W} q(w)$ and since $\operatorname{vol}(K)=1$,

$$
\operatorname{vol}(\mathcal{I})=\left(\sum_{w \in W} q(w)\right)^{-1}
$$

We have the following analog of [Cas95, Theorem 3.4]:

Claim 2.9. If $\alpha \in \Delta_{G_{n}}, w \in W$ and $\ell\left(s_{\alpha} w\right)>\ell(w)$,

$$
\begin{aligned}
& T_{s_{\alpha}} \varphi_{s_{\alpha} w, \chi}=\varphi_{w,{ }^{s} \alpha}+\left(c_{\alpha}(\chi)-q^{-1}\right) \varphi_{s_{\alpha} w,{ }^{s \alpha} \chi}, \\
& T_{s_{\alpha}} \varphi_{w, \chi}=\left(c_{\alpha}(\chi)-1\right) \varphi_{w,{ }^{s} \alpha}+q^{-1} \varphi_{s_{\alpha} w,{ }^{s} \chi} .
\end{aligned}
$$

Proof. Let $\mathfrak{w}_{\alpha}$ be the representative of $s_{\alpha}$. In the domain of absolute convergence

$$
T_{s_{\alpha}} \varphi_{e, \chi}\left(\mathfrak{s}\left(\mathfrak{w}_{\alpha}\right)\right)=\int_{N_{\alpha}} \varphi_{e, \chi}\left(\mathfrak{s}\left(\mathfrak{w}_{\alpha}\right)^{-1} \mathfrak{s}(v) \mathfrak{s}\left(\mathfrak{w}_{\alpha}\right)\right) d v
$$

The integrand vanishes unless $v \in N_{\alpha}(\varpi \mathcal{O})<K$, then $\mathfrak{s}\left(\mathfrak{w}_{\alpha}\right)^{-1} \mathfrak{s}(v) \mathfrak{s}\left(\mathfrak{w}_{\alpha}\right)=\kappa\left({ }^{\mathfrak{w}_{\alpha}^{-1}} v\right)$ and the integral equals $\operatorname{vol}(\varpi \mathcal{O})$. Hence

$$
T_{s_{\alpha}} \varphi_{e, \chi}\left(\mathfrak{s}\left(\mathfrak{w}_{\alpha}\right)\right)=q^{-1}
$$

This is true for all $\chi$ by meromorphic continuation. A similar computation gives

$$
T_{s_{\alpha}} \varphi_{s_{\alpha}, \chi}\left(\mathfrak{s}\left(I_{n}\right)\right)=\varphi_{s_{\alpha}, \chi}\left(\mathfrak{s}\left(\mathfrak{w}_{\alpha}\right)^{-1}\right)=1 .
$$

Here we used the fact that $\mathfrak{s}\left(\mathfrak{w}_{\alpha}\right)^{-1}=\mathfrak{s}\left(\mathfrak{w}_{\alpha}\right) \mathfrak{s}\left(t_{0}\right)$ for some $t_{0} \in T_{n} \cap K$. Exactly as in Cas95], the rank one case of (2.6) implies

$$
c_{\alpha}(\chi) \varphi_{K, s_{\alpha} \chi}=T_{s_{\alpha}} \varphi_{e, \chi}+T_{s_{\alpha}} \varphi_{s_{\alpha}, \chi}
$$

and we deduce

$$
\begin{aligned}
& T_{s_{\alpha}} \varphi_{s_{\alpha}, \chi}\left(\mathfrak{s}\left(\mathfrak{w}_{\alpha}\right)\right)=c_{\alpha}(\chi)-q^{-1}, \\
& T_{s_{\alpha}} \varphi_{e, \chi}\left(\mathfrak{s}\left(I_{n}\right)\right)=c_{\alpha}(\chi)-1 .
\end{aligned}
$$

If $w^{\prime} \in W-\left\{e, s_{\alpha}\right\}$ and $\mathfrak{w}^{\prime}$ is the representative of $w^{\prime}$,

$$
T_{s_{\alpha}} \varphi_{e, \chi}\left(\mathfrak{s}\left(\mathfrak{w}^{\prime}\right)\right)=T_{s_{\alpha}} \varphi_{s_{\alpha}, \chi}\left(\mathfrak{s}\left(\mathfrak{w}^{\prime}\right)\right)=0
$$


because $\mathfrak{w}_{\alpha}^{-1} N_{\alpha} \mathfrak{w}^{\prime}$ does not intersect with the support of the integrands. Combining this with the previous equalities,

$$
\begin{aligned}
& T_{s_{\alpha}} \varphi_{s_{\alpha}, \chi}=\varphi_{e,{ }^{s \alpha} \chi}+\left(c_{\alpha}(\chi)-q^{-1}\right) \varphi_{s_{\alpha},{ }^{s \alpha} \chi}, \\
& T_{s_{\alpha}} \varphi_{e, \chi}=\left(c_{\alpha}(\chi)-1\right) \varphi_{e,{ }^{s} \alpha}+q^{-1} \varphi_{s_{\alpha},{ }^{s} \alpha} .
\end{aligned}
$$

Now the assertions of the claim follow because if $\mathfrak{w}$ is the representative of $w$,

$$
q(w) \mathscr{P}_{\mathcal{I}}\left(\mathfrak{s}\left(\mathfrak{w}^{-1}\right) \varphi_{e, \chi}\right)=\varphi_{w, \chi}, \quad q(w) \mathscr{P}_{\mathcal{I}}\left(\mathfrak{s}\left(\mathfrak{w}^{-1}\right) \varphi_{s_{\alpha}, \chi}\right)=\varphi_{s_{\alpha} w, \chi}
$$

To see these formulas one can compute the integrals directly at $\mathfrak{s}(\mathfrak{w})\left(\right.$ resp. $\left.\mathfrak{s}\left(\mathfrak{w}_{\alpha} \mathfrak{w}\right)\right)$, then verify that for $\mathfrak{w}^{\prime} \neq \mathfrak{w}\left(\right.$ resp. $\left.\mathfrak{w}^{\prime} \neq \mathfrak{w}_{\alpha} \mathfrak{w}\right), \mathfrak{w}^{\prime} \mathcal{I} \mathfrak{w}^{-1}$ does not intersect $B_{n, *} \mathcal{I}\left(\right.$ resp. $\left.B_{n, *} \mathfrak{w}_{\alpha} \mathfrak{w} \mathcal{I}\right)$.

Combining (2.4) with (2.6) and the definition of the Casselman basis implies

$$
\varphi_{K, \chi}=\sum_{w \in W} c_{w}(\chi) f_{w, \chi}
$$

The $\widetilde{G}_{n}$-pairing on $\mathrm{I}(\chi) \times \mathrm{I}\left(\chi^{-1}\right)$ is given by (see [CO13, $\left.(10.2)\right]$ )

$$
\left\langle f_{\chi}, f_{\chi^{-1}}\right\rangle=\sum_{b \in B_{n, *} \backslash B_{n}} \delta_{B_{n}}^{-1}(b) \int_{K} f_{\chi}(\mathfrak{s}(b) \kappa(k)) f_{\chi^{-1}}(\mathfrak{s}(b) \kappa(k)) d k .
$$

The following result was proved in Cas80] in the course of computing Macdonald's formula ([Cas80, $\S 4])$.

Claim 2.10. For any $w \in W$,

$$
\mathscr{P}_{K}\left(f_{w, \chi}\right)(1)=\frac{c_{w_{0}}\left({ }^{w_{0} w} \chi\right)}{Q c_{w}(\chi)}, \quad Q=\sum_{w \in W} q(w)^{-1} .
$$

Proof. We prove the identity using the formula for the zonal spherical function. Put

$$
\Gamma_{\chi}(g)=\left\langle g \varphi_{K, \chi}, \varphi_{K, \chi^{-1}}\right\rangle, \quad g \in \widetilde{G}_{n} .
$$

In the non-metaplectic setting a formula for $\Gamma_{\chi}(g)$ was proved by MacDonald Mac95, Chapter $\mathrm{V}, \S 3$ ], then generalized to reductive groups in Cas80], as an application of the basis $\left\{f_{w, \chi}\right\}$. In the metaplectic case the formula was computed in [CO13, $\left.\S 10\right]$ and in particular for any $t \in C_{\widetilde{T}_{n}} \cap \widetilde{T}_{n}^{-}$,

$$
\Gamma_{\chi}(t)=Q^{-1} \sum_{w \in W} c_{w_{0}}\left({ }^{w_{0} w} \chi\right) \delta_{B_{n}}^{1 / 2}{ }^{w} \chi(t) .
$$

On the other hand using (2.13) and the fact that $\varphi_{K, \chi^{-1}}(\mathfrak{s}(b))=0$ unless $b \in B_{n, *}($ see (2.2) $)$,

$$
\begin{aligned}
\Gamma_{\chi}(g) & =\sum_{b \in B_{n, *} \backslash B_{n}} \delta_{B_{n}}^{-1}(b) \int_{K} \varphi_{K, \chi}(\mathfrak{s}(b) \kappa(k) g) \varphi_{K, \chi^{-1}}(\mathfrak{s}(b) \kappa(k)) d k \\
& =\int_{K} \varphi_{K, \chi}(\kappa(k) g) d k=\mathscr{P}_{K}\left(g \varphi_{K, \chi}\right)(1) .
\end{aligned}
$$

Putting (2.12) into this equality shows

$$
\Gamma_{\chi}(t)=\sum_{w \in W} c_{w}(\chi) \mathscr{P}_{K}\left(t f_{w, \chi}\right)(1) .
$$


Since $\mathscr{P}_{K}\left(t f_{w, \chi}\right)(1)=\mathscr{P}_{K}\left(\mathscr{P}_{\mathcal{I}}\left(t f_{w, \chi}\right)\right)(1)$, Claim 2.8 implies

$$
\Gamma_{\chi}(t)=\sum_{w \in W} c_{w}(\chi) \mathscr{P}_{K}\left(f_{w, \chi}\right)(1) \delta_{B_{n}}^{1 / 2}{ }^{w} \chi(t) .
$$

Comparing this with (2.14) and since the characters $\chi$ are linearly independent as functions on $t \in C_{\widetilde{T}_{n}} \cap \widetilde{T}_{n}^{-}$, we obtain the result.

We described the tools of Cas80] that we need and proceed to formulate an analog of Hironaka's theorem [Hir99, Proposition 1.9], for computing functionals on $\mathrm{I}(\chi)$.

2.3. Metaplectic version of Hironaka's theorem. Let $\mathrm{I}(\chi)^{*}$ denote the linear dual of $\mathrm{I}(\chi)$. For any $w \in W$, by definition

$$
T_{w^{-1},{ }^{w} \chi^{-1}}^{*}: \mathrm{I}\left(\chi^{-1}\right)^{*} \rightarrow \mathrm{I}\left({ }^{w} \chi^{-1}\right)^{*} .
$$

Since $\mathrm{I}(\chi)$ is the subspace of $\mathrm{I}\left(\chi^{-1}\right)^{*}$ of smooth functionals and $T_{w^{-1},{ }^{w} \chi^{-1}}$ preserves smoothness,

$$
T_{w^{-1},{ }^{w} \chi^{-1}}^{\vee}=\left.T_{w^{-1},{ }^{w} \chi^{-1}}\right|_{\mathrm{I}(\chi)}: \mathrm{I}(\chi) \rightarrow \mathrm{I}\left({ }^{w} \chi\right) .
$$

Claim 2.11. ([Hir99, Lemma 1.5, Proposition 1.6]) If $c_{w}(\chi) \neq 0$,

$$
T_{w^{-1},{ }^{w} \chi^{-1}}^{\vee}=\frac{c_{w^{-1}}\left({ }^{w} \chi^{-1}\right)}{c_{w}(\chi)} T_{w, \chi}
$$

If also $c_{w^{-1}}\left({ }^{w} \chi^{-1}\right) \neq 0$, the map

$$
\mathcal{T}_{w^{-1},{ }^{w} \chi^{-1}}=\frac{c_{w}(\chi)}{c_{w^{-1}}\left({ }^{w} \chi^{-1}\right)} T_{w^{-1},{ }^{w} \chi^{-1}}: \mathrm{I}\left(\chi^{-1}\right)^{*} \rightarrow \mathrm{I}\left({ }^{w} \chi^{-1}\right)^{*}
$$

extends $T_{w, \chi}$.

Proof. By (2.5) the mappings $T_{w^{-1}, w}^{\vee} \chi^{-1}$ and $T_{w, \chi}$ are proportional. To compute the proportionality factor, exactly as in [Hir99] we compare

$$
\left\langle T_{w, \chi}\left(\varphi_{K, \chi}\right), \varphi_{K,{ }^{w} \chi^{-1}}\right\rangle \text { to }\left\langle T_{w^{-1},{ }^{w} \chi^{-1}}^{\vee}\left(\varphi_{K, \chi}\right), \varphi_{K,{ }^{w} \chi^{-1}}\right\rangle
$$

using (2.6) and (2.13). The $\widetilde{G}_{n}$-pairing given by (2.13) involves a finite summation, but by (2.2) applied to $\varphi_{K,{ }^{w} \chi^{-1}}$, it reduces to one summand corresponding to the trivial coset.

As a corollary we obtain the following result, explaining the normalization factor used to define $\mathcal{T}_{w^{-1},{ }^{w} \chi^{-1}}$

Corollary 2.12. ([Hir99, Proposition 1.7]) If $c_{w}(\chi) c_{w^{-1}}\left({ }^{w} \chi^{-1}\right) \neq 0$, then for any compact open $K_{0}<K$,

$$
\mathscr{P}_{K_{0}} \circ \mathcal{T}_{w^{-1},{ }^{w} \chi^{-1}}=T_{w, \chi} \circ \mathscr{P}_{K_{0}} .
$$

Proof. Since $\mathscr{P}_{K_{0}}$ takes $\mathrm{I}\left(\chi^{-1}\right)^{*}$ to $\left(\mathrm{I}\left(\chi^{-1}\right)^{*}\right)^{K_{0}} \subset \mathrm{I}(\chi)$, both sides are mappings $\mathrm{I}\left(\chi^{-1}\right)^{*} \rightarrow$ $\mathrm{I}\left({ }^{w} \chi\right)$. Consider the left-hand side. Since $T_{w^{-1},{ }^{w} \chi^{-1}}$ and $\mathscr{P}_{K_{0}}$ commute, so do $\mathcal{T}_{w^{-1},{ }^{w} \chi^{-1}}$ and $\mathscr{P}_{K_{0}}$, but on the image of $\mathscr{P}_{K_{0}}$, according to Claim $2.11, \mathcal{T}_{w^{-1}{ }^{w} \chi^{-1}}$ extends $T_{w, \chi}$. 
Asssume we have a family $\left\{\Lambda_{a, \chi}\right\}_{a \in \mathcal{A}}$ of functionals $\Lambda_{a, \chi} \in \mathrm{I}\left(\chi^{-1}\right)^{*}$ indexed by a finite set $\mathcal{A}$, and such that for each $a, \Lambda_{a, \chi}$ is a meromorphic function in $\chi$. We fix an arbitrary ordering on $\mathcal{A}$. Furthermore, assume that for each $w \in W$ there is an invertible matrix $A(w, \chi)=\left(A(w, \chi)_{a, a^{\prime}}\right)$ such that

$$
\left(T_{w^{-1},{ }^{w} \chi^{-1}}^{*} \Lambda_{a, \chi}\right)_{a \in \mathcal{A}}=A(w, \chi)\left(\Lambda_{a,{ }^{w} \chi}\right)_{a \in \mathcal{A}} .
$$

The entries of $A(w, \chi)$ are by definition meromorphic in $\chi$.

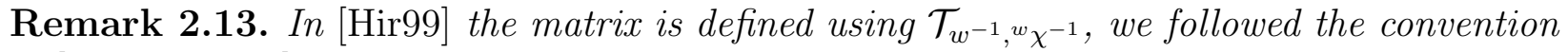
of [KP84, CO13].

For example, one can consider the family $\left\{\Upsilon_{w, b}\right\}$ of functionals introduced in the proof of Claim 2.6, then $A(w, \chi)$ exists because these form a basis of $\left(\mathrm{I}(\chi)_{N_{n}}\right)^{*}$. Another example is the Whittaker functionals, then $\mathcal{A}$ can be a set of representatives of $T_{n, *} \backslash T_{n}$ (see [KP84, CO13]).

We are interested in obtaining a formula for $\Lambda_{a, \chi}\left(g \varphi_{K, \chi^{-1}}\right)$, where $g \in \widetilde{G}_{n}$. Since $\mathscr{P}_{\mathcal{I}}\left(g^{-1} \Lambda_{a, \chi}\right) \in$ $\left(\mathrm{I}\left(\chi^{-1}\right)^{*}\right)^{\mathcal{I}} \subset \mathrm{I}(\chi)^{\mathcal{I}}$, there is a function $\lambda_{a, \chi, g} \in \mathrm{I}(\chi)^{\mathcal{I}}$ satisfying

$$
\mathscr{P}_{I}\left(g^{-1} \Lambda_{a, \chi}\right)\left(f_{\chi^{-1}}^{\prime}\right)=\left\langle\lambda_{a, \chi, g}, f_{\chi^{-1}}^{\prime}\right\rangle, \quad \forall f_{\chi^{-1}}^{\prime} \in \mathrm{I}\left(\chi^{-1}\right) .
$$

In other words $\lambda_{a, \chi, g}$ is the image of $\mathscr{P}_{I}\left(g^{-1} \Lambda_{a, \chi}\right)$ in $\mathrm{I}(\chi)$.

Claim 2.14. $\Lambda_{a, \chi}\left(g \varphi_{K, \chi^{-1}}\right)=\mathscr{P}_{K}\left(\lambda_{a, \chi, g}\right)(1)$.

Proof. Equality (2.16) together with (2.13) and (2.2) imply

$$
\mathscr{P}_{I}\left(g^{-1} \Lambda_{a, \chi}\right)\left(\varphi_{K, \chi^{-1}}\right)=\mathscr{P}_{K}\left(\lambda_{a, \chi, g}\right)(1) \text {. }
$$

Then since

$$
\Lambda_{a, \chi}\left(g \varphi_{K, \chi^{-1}}\right)=g^{-1} \Lambda_{a, \chi}\left(\varphi_{K, \chi^{-1}}\right)=\mathscr{P}_{I}\left(g^{-1} \Lambda_{a, \chi}\right)\left(\varphi_{K, \chi^{-1}}\right),
$$

the result holds.

Here is the analog of [Hir99, Proposition 1.9].

Lemma 2.15.

$$
\left(\Lambda_{a, \chi}\left(g \varphi_{K, \chi^{-1}}\right)\right)_{a \in \mathcal{A}}=\sum_{w \in W} \frac{c_{w_{0}}\left({ }^{w_{0} w} \chi\right)}{Q c_{w^{-1}}\left({ }^{w} \chi^{-1}\right)} A(w, \chi)\left(\lambda_{a,{ }^{w} \chi, g}(1)\right)_{a \in \mathcal{A}} .
$$

Proof. The formal steps in [Hir99] can be carried out immediately, because we established the machinery used in loc. cit. for the metaplectic setting. We repeat the steps, for readability. For the proof we may assume $c_{w}(\chi) c_{w^{-1}}\left({ }^{w} \chi^{-1}\right) \neq 0$. Since $\lambda_{a, \chi, g} \in \mathrm{I}(\chi)^{\mathcal{I}}$, we can write

$$
\lambda_{a, \chi, g}=\sum_{w \in W} d_{a, \chi}(g) f_{w, \chi}
$$

for some complex coefficients $d_{a, \chi}(g)$. The coefficients can be computed by applying $\Upsilon_{w, \chi}$,

$$
d_{a, \chi}(g)=T_{w, \chi} \lambda_{a, \chi, g}(1) .
$$

By the definition of $\lambda_{a, \chi, g}$ and using Corollary 2.12 ,

$$
T_{w, \chi} \lambda_{a, \chi, g}=T_{w, \chi} \mathscr{P}_{I}\left(g^{-1} \Lambda_{a, \chi}\right)=\mathscr{P}_{\mathcal{I}}\left(\mathcal{T}_{w^{-1},{ }^{w} \chi^{-1}} g^{-1} \Lambda_{a, \chi}\right) .
$$

Then Claim 2.11 implies

$$
d_{a, \chi}(g)=\mathscr{P}_{\mathcal{I}}\left(\mathcal{T}_{w^{-1},{ }^{w} \chi^{-1}} g^{-1} \Lambda_{a, \chi}\right)(1)=\frac{c_{w}(\chi)}{c_{w^{-1}}\left({ }^{w} \chi^{-1}\right)} \mathscr{P}_{\mathcal{I}}\left(g^{-1} T_{w^{-1},{ }^{w} \chi^{-1}} \Lambda_{a, \chi}\right)(1) .
$$


By Claim 2.14, to compute $\Lambda_{a, \chi}\left(g \varphi_{K, \chi^{-1}}\right)$ we apply $\mathscr{P}_{K}$ to (2.17) and evaluate at 1 . Claim 2.10 gives the values of $\mathscr{P}_{K}\left(f_{w, \chi}\right)(1)$. We obtain

$$
\Lambda_{a, \chi}\left(g \varphi_{K, \chi^{-1}}\right)=\sum_{w \in W} \mathscr{P}_{K}\left(f_{w, \chi}\right)(1) d_{a, \chi}(g)=\sum_{w \in W} \frac{c_{w_{0}}\left({ }^{w_{0} w} \chi\right)}{Q c_{w^{-1}}\left({ }^{w} \chi^{-1}\right)} \mathscr{P}_{\mathcal{I}}\left(g^{-1} T_{w^{-1},{ }^{w} \chi^{-1}}^{*} \Lambda_{a, \chi}\right)(1) .
$$

Now since

$$
\mathscr{P}_{\mathcal{I}}\left(g^{-1} T_{w^{-1},{ }^{w} \chi^{-1}}^{*} \Lambda_{a, \chi}\right)=T_{w^{-1},{ }^{w} \chi^{-1}}^{*} \mathscr{P}_{\mathcal{I}}\left(g^{-1} \Lambda_{a, \chi}\right)
$$

(2.15) implies

$$
\left(\Lambda_{a, \chi}\left(g \varphi_{K, \chi^{-1}}\right)\right)_{a \in \mathcal{A}}=\sum_{w \in W} \frac{c_{w_{0}}\left({ }^{w_{0} w} \chi\right)}{Q c_{w^{-1}}\left({ }^{w} \chi^{-1}\right)} A(w, \chi)\left(\lambda_{a,{ }^{w} \chi, g}(1)\right)_{a \in \mathcal{A}}
$$

as claimed.

As observed in [Sak06], the value of $\lambda_{a, \chi, g}(1)$ corresponds to the evaluation of the functional at $g \varphi_{e, \chi^{-1}}$. Namely,

Claim 2.16. $\lambda_{a, \chi, g}(1)=\operatorname{vol}(\mathcal{I})^{-1} \Lambda_{a, \chi}\left(g \varphi_{e, \chi^{-1}}\right)$.

Proof. We show

$$
\lambda_{a, \chi, g}(1)=\operatorname{vol}(\mathcal{I})^{-1}\left\langle\lambda_{a, \chi, g}, \varphi_{e, \chi^{-1}}\right\rangle .
$$

Then the result follows from (2.16) because

$$
\mathscr{P}_{I}\left(g^{-1} \Lambda_{a, \chi}\right)\left(\varphi_{e, \chi^{-1}}\right)=\Lambda_{a, \chi}\left(g \varphi_{e, \chi^{-1}}\right) .
$$

For $b \in B_{n}$ and $k \in K$, if $\mathfrak{s}(b) \kappa(k)$ belongs to the support of $\varphi_{e, \chi^{-1}}$, then $b \in B_{n, *}$ and $k \in \mathcal{I}$. This is because when $t T_{n, *} \neq t^{\prime} T_{n, *}$, the sets $t B_{n, *} K$ and $t^{\prime} B_{n, *} K$ are disjoint and $K \cap B_{n, *} \mathcal{I}=\mathcal{I}$ (use (2.1)). Hence looking at (2.13) we obtain

$$
\left\langle\lambda_{a, \chi, g}, \varphi_{e, \chi^{-1}}\right\rangle=\int_{\mathcal{I}} \lambda_{a, \chi, g}(\kappa(k)) d k=\operatorname{vol}(\mathcal{I}) \lambda_{a, \chi, g}(1),
$$

as required.

\section{Corollary 2.17.}

$$
\left(\Lambda_{a, \chi}\left(g \varphi_{K, \chi^{-1}}\right)\right)_{a \in \mathcal{A}}=\frac{1}{Q \operatorname{vol}(\mathcal{I})} \sum_{w \in W} \frac{c_{w_{0}}\left({ }^{w_{0} w} \chi\right)}{c_{w^{-1}}\left({ }^{w} \chi^{-1}\right)} A(w, \chi)\left(\Lambda_{a,{ }^{w} \chi}\left(g \varphi_{e, \chi^{-1}}\right)\right)_{a \in \mathcal{A}} .
$$

As mentioned in the introduction, the proofs in $\S 2.22 .3$ apply to a more general setting of covering groups. The preliminary results we used are essentially the Gindikin-Karpelevich formula (2.6), the description of Casselman's basis using intertwining operators (in the proof of Claim 2.5) and a formula for the zonal spherical function (used for the proof Claim 2.10). These results were established in [CO13] for $r$-fold covers of $G_{n}$, hence our results apply to these groups as well.

McNamara has generalized substantially several results of [CO13, to any central extension of $G$ by a finite cyclic group, where $G$ is an unramified reductive group over a $p$-adic field. See [McN11, Theorem 6.4] regarding the Gindikin-Karpelevich formula and [McN14, Proposition 5.2] (Casselman's basis). As mentioned in [McN14, the zonal spherical formula can also be proved using his tools. To be cautious, we refrain from stating our results in this generality. 


\section{The metaplectic Shalika model}

3.1. Definition. Let $F$ be a local field and $\psi$ be a nontrivial additive character of $F$. First we recall the notion of the Shalika model, introduced by Jacquet and Shalika [JS90].

Put $n=2 k$. Consider the unipotent radical $U_{k}$ of the parabolic subgroup $Q_{k}<G_{n}$, and the (Shalika) character $\psi$ of $U_{k}$ given by

$$
\psi\left(\left(\begin{array}{cc}
I_{k} & u \\
& I_{k}
\end{array}\right)\right)=\psi(\operatorname{tr}(u)) .
$$

The normalizer of $U_{k}$ and stabilizer of $\psi$ is

$$
G_{k}^{\triangle}=\left\{c^{\triangle}: c \in G_{k}\right\}, \quad c^{\triangle}=\operatorname{diag}(c, c) .
$$

The group $G_{k}^{\triangle} \ltimes U_{k}$ is called the Shalika group. Let $\pi \in \operatorname{Alg} G_{n}$. A $\psi$-Shalika functional on the space of $\pi$ is a functional $l$ such that for any vector $\xi$ in the space of $\pi, c \in G_{k}$ and $u \in U$,

$$
l\left(\pi\left(c^{\triangle} u\right) \xi\right)=\psi(u) l(\xi) .
$$

Here on the left-hand side $u=\left(\begin{array}{cc}I_{k} & u \\ & I_{k}\end{array}\right)$. If the field is Archimedean we also demand that $l$ is continuous.

Jacquet and Rallis JR96] proved that over a non-Archimedean field, when $\pi$ is irreducible, the space of such functionals is at most one-dimensional. First they proved that $\left(G_{n}, M_{k}\right)$ is a Gelfand pair, then they used analytic techniques similar to [FJ93] to show that a Shalika functional induces an $M_{k}$-invariant functional. Their result was extended to Archimedean fields in AGJ09.

We mention that Ash and Ginzburg [AG94] already proved uniqueness results for a certain class of irreducible unramified representations, we will adapt their proof to the metaplectic case (see Theorem 3.10 below).

Granted that $l$ exists, one may define the Shalika model $\mathscr{S}(\pi, \psi)$ of $\pi$ as the space of functions $\mathscr{S}_{\xi}(g)=l(\pi(g) \xi)$, as $\xi$ varies in the space of $\pi$.

We explain the metaplectic analog (following Kap15a]). According to (1.1), restriction of the cover of $G_{n}$ to $G_{k}^{\triangle}$ is a "simple cover" in the sense that $\sigma\left(c^{\triangle}, c^{\prime}\right)^{\prime}=\left(\operatorname{det} c, \operatorname{det} c^{\prime}\right)_{2}$. Hence

$$
\mathfrak{s}\left(\left(c c^{\prime}\right)^{\triangle}\right)=\sigma\left(c^{\triangle}, c^{\prime \triangle}\right) \mathfrak{s}\left(c^{\triangle}\right) \mathfrak{s}\left(c^{\prime \triangle}\right)=\left(\operatorname{det} c, \operatorname{det} c^{\prime}\right)_{2} \mathfrak{s}\left(c^{\triangle}\right) \mathfrak{s}\left(c^{\prime \triangle}\right) .
$$

Then if $\psi^{\prime}$ is another nontrivial additive character of $F$, any genuine representation of $\widetilde{G}_{k}^{\triangle}$ takes the form $\pi \otimes \gamma_{\psi^{\prime}}$, where $\pi$ is a representation of $G_{k}$ and

$$
\pi \otimes \gamma_{\psi^{\prime}}\left(\epsilon \mathfrak{s}\left(c^{\triangle}\right)\right)=\epsilon \gamma_{\psi^{\prime}}(\operatorname{det} c) \pi(c), \quad \epsilon \in \mu_{2}
$$

For a genuine $\pi \in \operatorname{Alg} \widetilde{G}_{n}$, we call $l$ a metaplectic $\left(\psi^{\prime}, \psi\right)$-Shalika functional on $\pi$ if

$$
l\left(\pi\left(\mathfrak{s}\left(c^{\triangle} u\right)\right) \xi\right)=\gamma_{\psi^{\prime}}(\operatorname{det} c) \psi(u) l(\xi) .
$$

The terminology is reasonable because $\gamma_{\psi^{\prime}}$ corresponds to the trivial character of $G_{k}$. If $\pi$ is irreducible, we denote the corresponding metaplectic Shalika model by $\mathscr{S}\left(\pi, \psi^{\prime}, \psi\right)$. It is a subrepresentation of $\operatorname{Ind}_{\widetilde{G}_{k}^{\triangle} U_{k}}^{\widetilde{G}_{n}}\left(\gamma_{\psi^{\prime}} \otimes \psi\right)$, where $\gamma_{\psi^{\prime}} \otimes \psi\left(\epsilon \mathfrak{s}\left(c^{\triangle} u\right)\right)=\epsilon \gamma_{\psi^{\prime}}(\operatorname{det} c) \psi(u)$.

Remark 3.1. Since $\operatorname{det} c^{\triangle} \in F^{* 2}$, we also have $\sigma^{(1)}\left(c^{\triangle}, c^{\prime}\right)=\left(\operatorname{det} c \text {, } \operatorname{det} c^{\prime}\right)_{2}$ and (3.1) also holds for the section corresponding to $\sigma^{(1)}$. (The cocycle $\sigma^{(1)}$ was defined in $\S$ 1.2. as we mentioned there our work applies to both covers.) 
We have the following bound for Shalika functions.

Claim 3.2. Assume that $\pi \in \mathrm{Alg} \widetilde{G}_{n}$ is genuine irreducible and admits a metaplectic Shalika model. Then there exists $\alpha>0$ such that for any $\xi$ in the space of $\pi$, there exists a positive $\phi \in \mathcal{S}\left(F_{k \times k}\right)$ such that

$$
\left|\mathscr{S}_{\xi}\left(\mathfrak{s}\left(\operatorname{diag}\left(g, I_{k}\right)\right)\right)\right| \leq|\operatorname{det} g|^{-\alpha} \phi(g), \quad \forall g \in G_{k} .
$$

Proof. Over a $p$-adic field, the proof is a simple adaptation of Lemma 6.1 of Jacquet and Rallis JR96 for the non-metaplectic setting. The main difference is that the asymptotic expansion is written for $T_{k}^{2}$ instead of $T_{k}$, the group $T_{k}^{2}$ is abelian and splits under the cover.

If $F$ is Archimedean, by the Dixmier-Malliavin Lemma applied to the representation $\left.\pi\right|_{U_{k}}$ there exist $\xi_{j}$ in the representation space of $\pi$ and $\phi_{j} \in \mathcal{S}\left(U_{k}\right)$ such that $\xi=\sum_{j} \theta\left(\phi_{j}\right) \xi_{j}$. This implies that for $d_{g}=\mathfrak{s}\left(\operatorname{diag}\left(g, I_{k}\right)\right)$ we have

$$
\mathscr{S}_{\xi}\left(d_{g}\right)=\sum_{j} \int_{U_{k}} \phi_{j}(u) \mathscr{S}_{\xi_{j}}\left(d_{g} \mathfrak{s}(u)\right) d u=\sum_{j} \mathscr{S}_{\xi_{j}}\left(d_{g}\right) \int_{U_{k}} \phi_{j}(u) \psi(g u) d u=\sum_{j} \widehat{\phi}_{j}(g) \mathscr{S}_{\xi_{j}}\left(d_{g}\right) \text {. }
$$

It remains to show that each term $\mathscr{S}_{\xi_{j}}\left(d_{g}\right)$ is bounded by a constant times $|\operatorname{det} g|^{-\alpha}$ for some $\alpha$ independent of $\xi_{j}$. This follows from [AGJ09, Lemma 3.2], which also holds in the metaplectic setting since the proof merely uses the Lie algebra action.

The next claim is a simple analog of the relation between Whittaker and Kirillov models (see [GK75, Proposition 2]).

Claim 3.3. Assume $F$ is non-Archimedean. Let $\pi$ be a genuine irreducible subrepresentation of $\operatorname{Ind}_{\widetilde{G}_{k}^{\triangle} U_{k}}^{\widetilde{G}_{n}}\left(\gamma_{\psi^{\prime}} \otimes \psi\right)$. The restriction of functions in the space of $\pi$ to $\widetilde{Q}_{n}$ contains any element of $\operatorname{ind}_{\widetilde{G}_{k}^{\triangle} U_{k}}^{\widetilde{Q}_{n}}\left(\gamma_{\psi^{\prime}} \otimes \psi\right)$.

Proof. For any $\xi$ in the space of $\pi$ and $\phi \in \mathcal{S}\left(U_{k}\right)$, the function $\phi(\xi)$ defined by

$$
\phi(\xi)(x)=\int_{U_{k}} \phi(u) \xi(x \mathfrak{s}(u)) d u, \quad x \in \widetilde{G}_{n}
$$

belongs to the space of $\pi$.

Given $d_{g}=\mathfrak{s}\left(\operatorname{diag}\left(g, I_{k}\right)\right) \in \widetilde{Q}_{n}$, one can find $\xi$ such that $\xi\left(d_{g}\right) \neq 0$ and we also have $\phi(\xi)\left(d_{g}\right)=\widehat{\phi}(g) \xi\left(d_{g}\right)$, where $\widehat{\phi}$ denotes the Fourier transform of $\phi$ with respect to $\psi$. Note that the group of characters of $U_{k}$ is isomorphic to the direct product of $k^{2}$ copies of $F$. We can select $\phi$ such that the support of $\widehat{\phi}$ is contained in $g \mathcal{V}$, where $\mathcal{V}<G_{k}$ is a small compact open neighborhood of the identity. Then $\left.\phi(\xi)\right|_{\widetilde{Q}_{n}}$ belongs to ind ${\widetilde{\widetilde{G}_{k}}}_{k}^{\widetilde{Q}_{k}}{ }_{U_{k}}\left(\gamma_{\psi^{\prime}} \otimes \psi\right)$. The latter space is spanned by these functions, as $g$ and $\mathcal{V}$ vary.

3.2. Asymptotic expansion of metaplectic Shalika functions. As with Whittaker functions, one can write an asymptotic expansion for Shalika functions, as functions on the torus. Over $p$-adic fields, such an expansion was obtained by Jacquet and Rallis [JR96, $\S 6.2$ ] and their result can be easily extended to the metaplectic setting. Over Archimedean fields, as a rule, the problem is more difficult, because one has to deal with delicate continuity properties. Here we provide an asymptotic expansion for metaplectic Shalika functions over $F=\mathbb{R}$ or $\mathbb{C}$. In particular, the same result holds in the non-metaplectic setting for the Shalika functional. 
Remark 3.4. The results of this section will be used in $\S 5$ to prove the meromorphic continuation of the local integrals (see the proof of Theorem 5.3).

Let $n=2 k$. We use the notation and definitions of $\S\left[1.5\right.$, e.g., $A_{\ell}, a_{x}, E_{1}\left(Q_{\ell}, \pi\right)$ and $\Lambda_{\pi, \ell}$, where now $\pi \in \operatorname{Alg} \widetilde{G}_{n}$ is a genuine representation. Note that $a_{x}$ is viewed as an element in $G_{n}$ rather than $G_{k}$ by embedding $G_{k}$ into $G_{n}$ via $g \mapsto \operatorname{diag}\left(g, I_{k}\right)$.

Theorem 3.5. Let $\pi \in \operatorname{Alg} \widetilde{G}_{n}$ be a genuine irreducible representation on a space $V$, that admits a metaplectic Shalika functional $l$. Then for all $D=\left(D_{1}, \ldots, D_{k}\right) \in \mathbb{R}^{k}$ there exist finite subsets $C_{\ell} \subset E^{(\ell)}(\pi)$ and functions $p_{I, z}: \mathbb{R}^{k} \times V \rightarrow \mathbb{C}$ for $I \subset\{1, \ldots, k\}, z \in \prod_{\ell \in I} C_{\ell}$, such that the following holds.

(1) For all $\xi \in V$ and $x \in \mathbb{R}^{k}$,

$$
l\left(\pi\left(\mathfrak{s}\left(a_{x}\right)\right) \xi\right)=\sum_{I, z} p_{I, z}(x ; \xi) \cdot e^{-z \cdot x_{I}}
$$

where the (finite) summation is over all $I \subset\{1, \ldots, k\}$ and $z \in \prod_{\ell \in I} C_{\ell}$.

(2) Each function $p_{I, z}(x ; \xi)$ is polynomial in $x_{I}$ :

$$
p_{I, z}(x ; \xi)=\sum_{\alpha \in \mathbb{N}^{I}} c_{\alpha}\left(x_{\bar{I}} ; \xi\right) x_{I}^{\alpha},
$$

where the coefficients $c_{\alpha}\left(x_{\bar{I}} ; \xi\right)$ are smooth in $x_{\bar{I}}$, linear in $\xi$, and satisfy bounds of the form

$$
\left|c_{\alpha}\left(x_{\bar{I}} ; \xi\right)\right| \leq q(\xi)\left(1+\left|x_{\bar{I}}\right|\right)^{d} e^{-D_{\bar{I}} \cdot x_{\bar{I}}}, \quad \forall x_{\bar{I}} \in \mathbb{R}_{>0}^{\bar{I}}, \xi \in V,
$$

with $d \geq 0$ and a continuous seminorm $q$ on $V$.

Proof. The proof is similar to the proof of Theorem 1.1, we explain the necessary modifications. The main difference is, of course, that $l$ is not a smooth functional.

In contrast to the situation in Theorem 1.1 we cannot replace $l$ by $\pi^{\vee}(X) l$ for $X \in \mathcal{U}\left(\mathfrak{g}_{n}\right)$ since in general $\pi^{\vee}(X) l$ is not a metaplectic Shalika functional. This replacing was used in Theorem 1.1 for elements $X \in \mathfrak{u}_{\ell}, 1 \leq \ell \leq k$ (in Theorem 1.1 everything depended continuously on the funtional). However, each $X \in \mathfrak{u}_{\ell}$ can be written as $X=X_{1}+X_{2}$ with $X_{1} \in \mathfrak{u}_{k}$ and $X_{2}=\operatorname{diag}(Y, 0)$ for some $Y \in \mathfrak{g}_{k}$ (here 0 denotes the $k \times k$ zero matrix). By (3.2) we obtain

$$
\begin{aligned}
l\left(\pi(X) \pi\left(\mathfrak{s}\left(a_{x}\right)\right) \xi\right) & =l\left(\pi\left(X_{1}\right) \pi\left(\mathfrak{s}\left(a_{x}\right)\right) \xi\right)+l\left(\pi(\operatorname{diag}(Y, 0)) \pi\left(\mathfrak{s}\left(a_{x}\right)\right) \xi\right) \\
& =\psi\left(X_{1}\right) l\left(\pi\left(\mathfrak{s}\left(a_{x}\right)\right) \xi\right)-l\left(\pi(\operatorname{diag}(0, Y)) \pi\left(\mathfrak{s}\left(a_{x}\right)\right) \xi\right) \\
& =\psi\left(X_{1}\right) l\left(\pi\left(\mathfrak{s}\left(a_{x}\right)\right) \xi\right)-l\left(\pi\left(\mathfrak{s}\left(a_{x}\right)\right) \pi(\operatorname{diag}(0, Y)) \xi\right) .
\end{aligned}
$$

In the second term we can replace $\pi(\operatorname{diag}(0, Y)) \xi$ by $\xi$ since the map $\xi \mapsto \pi(\operatorname{diag}(0, Y)) \xi$ is continuous and all data in the statement depends continuously on $\xi$. This shows that the recursive argument in the proof of Theorem 1.1 extends to our setting.

It remains to show that an estimate of the form (1.4) also holds for $l$. More precisely, we show that there exists a continuous seminorm $q$ on $V$ and $d \geq 0$ such that

$$
\left|l\left(\pi\left(\mathfrak{s}\left(a_{x}\right)\right) \xi\right)\right| \leq q(\xi)(1+|x|)^{d} e^{-\Lambda_{\pi} \cdot x}, \quad \forall x \in \mathbb{R}_{>0}^{k}, \xi \in V .
$$

First, $l$ is a continuous linear functional on $V$, so there exists a continuous seminorm $q_{1}$ on $V$ such that

$$
|l(\xi)| \leq q_{1}(\xi), \quad \forall \xi \in V
$$


Since $\pi$ is of moderate growth there exists $N \geq 0$ and a continuous seminorm $q_{2}$ on $V$ such that

$$
q_{1}(\pi(g) \xi) \leq q_{2}(\xi)\|g\|^{N}, \quad \forall g \in \widetilde{G}_{n}, \xi \in V,
$$

where $\|\cdot\|$ is the pullback to $\widetilde{G}_{n}$ of the usual norm function on $G_{n}$, i.e.,

$$
\|h\|=\sum_{i, j=1}^{n}\left(\left|h_{i, j}\right|^{2}+\left|\left(h^{-1}\right)_{i, j}\right|^{2}\right), \quad h \in G_{n} .
$$

For $x \in \mathbb{R}_{>0}^{k}$ we clearly have

$$
\left\|a_{x}\right\|^{N} \leq C e^{D \cdot x}, \quad \forall x \in \mathbb{R}_{>0}^{k}
$$

for some $C>0$ and $D=\left(D_{1}, \ldots, D_{k}\right) \in \mathbb{R}^{k}$. For $q=C q_{2}$,

$$
\left|l\left(\pi\left(\mathfrak{s}\left(a_{x}\right)\right) \xi\right)\right| \leq q(\xi) e^{D \cdot x}, \quad \forall x \in \mathbb{R}_{>0}^{k}, \xi \in V .
$$

We now argue as in Wal88, Section 4.3.5]. More precisely, applying the recursive argument in the proof of Theorem [1.1 shows that after possibly multiplying with a power of $(1+|x|)$, we can replace $D$ by $D-e_{\ell}$ for any $1 \leq \ell \leq k$ as long as $D_{\ell}-1 \geq-\Lambda_{\pi, \ell}$. Repeating this shows that $D$ can actually be replaced by $-\Lambda_{\pi}$ after multiplying with a certain power of $(1+|x|)$, and hence we obtain (3.6) $)$.

Remark 3.6. As stated, the estimates for $l\left(\pi\left(\mathfrak{s}\left(a_{x}\right)\right) \xi\right)$ in Theorems 1.1 and 3.5 only hold for $x \in \mathbb{R}_{>0}^{k}$. However, one can also expand $l\left(\pi\left(\mathfrak{s}\left(a_{x}\right)\right) \xi\right)$ for $x_{1}, \ldots, x_{k-1} \geq 0$ and $x_{k} \leq 0$ into an exponential power series, and the remainder terms are at least bounded by $e^{-N x_{k}}$ for some $N \geq 0$. To see this, first note that $l\left(\pi\left(\mathfrak{s}\left(a_{x}\right)\right) \xi\right)=l\left(\pi\left(\mathfrak{s}\left(a_{x^{\prime}}\right)\right) \pi\left(\mathfrak{s}\left(e^{-x_{k} H_{k}}\right)\right) \xi\right)$ where $x^{\prime}=\left(x_{1}, \ldots, x_{k-1}, 0\right)$. Then apply the expansion with $\xi$ replaced by $\pi\left(\mathfrak{s}\left(e^{-x_{k} H_{k}}\right)\right) \xi$, and use the fact that for each seminorm $q$ there exists another seminorm $q^{\prime}$ and $N \geq 0$ such that $q\left(\pi\left(\mathfrak{s}\left(e^{-x_{k} H_{k}}\right)\right) \xi\right) \leq q^{\prime}(\xi) e^{-N x_{k}}$ for all $x_{k} \leq 0$ (see the proof of Theorem 3.5 ).

3.3. The unramified metaplectic Shalika function. In this section we develop an explicit formula for the unramified metaplectic Shalika function. Our arguments closely follow those of Sakellaridis Sak06], who established this formula in the non-metaplectic setting. We use the definitions and results of $\S 2$, in particular $|2|=1$ and we have the canonical splitting $\kappa$ of $K$ as in $\S 2.1$.

Assume $n=2 k$. Let $\mathrm{I}(\chi)$ be a genuine unramified principal series representation, $\chi=\chi_{\underline{s}}$ (since $n$ is even, $\gamma$ can be ignored). Let $\psi$ and $\psi^{\prime}$ be a pair of nontrivial additive characters of $F$, and assume they are both unramified, i.e., their conductor is $\mathcal{O}$. Assuming that $\underline{s}$ is in a "suitable general position", we construct a metaplectic $\left(\psi^{\prime}, \psi\right.$ )-Shalika functional (see $\S 3.1$ for the definition) on $\mathrm{I}(\chi)$, and provide an explicit formula for the value of such a functional on the normalized unramified element of $\mathrm{I}(\chi)$.

Identify $\Delta_{G_{n}}$ with the pairs $(i, i+1), 1 \leq i<n$, and put $\alpha_{i}=(i, i+1)$, then $\Sigma_{G_{n}}$ consists of the pairs $(i, j)$ with $1 \leq i \neq j \leq n$. We will need a few simple auxiliary results for computing conjugations.

Claim 3.7. Let $\mathfrak{w} \in \mathfrak{W}$ and $t \in T_{n}$. Assume that $\mathfrak{w}$ is the representative of $w \in W$. Then $\mathfrak{s}(\mathfrak{w}) \mathfrak{s}(t)=\sigma(\mathfrak{w}, t) \mathfrak{s}\left({ }^{\mathfrak{w}} t\right)$, where

$$
\sigma(\mathfrak{w}, t)=\prod_{\{(i, j)=\alpha>0: w \alpha<0\}}\left(-t_{j}, t_{i}\right) .
$$


Proof. Since $\mathfrak{s}(\mathfrak{w}) \mathfrak{s}(t) \mathfrak{s}(\mathfrak{w} t)^{-1}=\sigma(\mathfrak{w}, t)$,

$$
\mathfrak{s}(\mathfrak{w}) \mathfrak{s}(t) \mathfrak{s}(\mathfrak{w})^{-1}=\sigma(\mathfrak{w}, t) \mathfrak{s}(\mathfrak{w} t) \mathfrak{s}(\mathfrak{w})^{-1}=\sigma(\mathfrak{w}, t) \mathfrak{s}\left({ }^{\mathfrak{w}} t \mathfrak{w}\right) \mathfrak{s}(\mathfrak{w})^{-1}=\sigma(\mathfrak{w}, t) \mathfrak{s}\left({ }^{\mathfrak{w}} t\right) .
$$

For the last equality we used the fact that $\sigma\left(t^{\prime}, \mathfrak{w}\right)=1$ for any $t^{\prime} \in T_{n}$ ([BLS99, $\S 3$, Theorem 7]). The value of $\sigma(\mathfrak{w}, t)$ was given in [BLS99, $\S 3$, Lemma 3].

For any $t, t^{\prime} \in T_{n}$, according to the computation of $\sigma\left(t, t^{\prime}\right)$ in [BLS99, $\S 3$, Lemma 1],

$$
\left[\mathfrak{s}(t), \mathfrak{s}\left(t^{\prime}\right)\right]=\mathfrak{s}(t) \mathfrak{s}\left(t^{\prime}\right) \mathfrak{s}(t)^{-1} \mathfrak{s}\left(t^{\prime}\right)^{-1}=\prod_{i<j}\left(t_{i}, t_{j}^{\prime}\right)_{2}\left(t_{i}^{\prime}, t_{j}\right)_{2} .
$$

Remark 3.8. Let $w$ be a permutation matrix. One can find unique $\mathfrak{w} \in \mathfrak{W}$ and $t_{0} \in T_{n}$ such that $w=t_{0} \mathfrak{w}$, where the diagonal coordinates of $t_{0}$ are \pm 1 . Then for any $t \in T_{n}$, $\mathfrak{s}(w) \mathfrak{s}(t)={ }^{\mathfrak{s}\left(t_{0}\right)}\left({ }^{\mathfrak{s}(\mathfrak{w})} \mathfrak{s}(t)\right)$, which can be computed using Claim 3.7 and (3.7).

Corollary 3.9. Let $w$ be a permutation matrix and write $w=t_{0} \mathfrak{w}$ as in Remark 3.8. For any $t \in T_{n, *},{ }^{\mathfrak{s}(w)} \mathfrak{s}(t)={ }^{\mathfrak{s}(\mathfrak{w})} \mathfrak{s}(t)$. If $\mathfrak{s}(t) \in C_{\widetilde{T}_{n}},{ }^{\mathfrak{s}(w)} \mathfrak{s}(t)=\mathfrak{s}\left({ }^{\mathfrak{w}} t\right)$.

The following theorem shows that when the inducing data is in a certain general position, which does not preclude reducibility, the space of metaplectic Shalika functionals is at most one-dimensional. Ash and Ginzburg [AG94, Lemma 1.7] proved this for $G_{n}$. Our proof follows theirs, with certain modifications.

For $1 \leq i<j \leq n$ and $x \in F^{*}$, let $t_{i, j}(x)=\mathfrak{s}\left(\operatorname{diag}\left(I_{i-1}, x^{2}, I_{j-i-1}, x^{2}, I_{n-j}\right)\right) \in C_{\widetilde{T}_{n}}$.

Theorem 3.10. Assume that $\chi$ satisfies the following conditions.

(1) $\chi\left(t_{i, j}\right) \neq 1$ for all $1 \leq i<j \leq k$.

(2) There exists a permutation $\tau$ on $\{1, \ldots, k\}$ such that $\chi\left(t_{i, k+j}\right) \neq 1$ for all $1 \leq i, j \leq k$ such that $j \neq \tau(i)$.

The space of metaplectic Shalika functionals on $\mathrm{I}(\chi)$ is at most one-dimensional. Furthermore, it is zero dimensional unless $\chi\left(t_{i, k+\tau(i)}\right)=1$ for all $1 \leq i \leq k$.

Proof. For the first assertion we have to show

$$
\operatorname{dim} \operatorname{Hom}_{\widetilde{G}_{n}}\left(\mathrm{I}(\chi), \operatorname{Ind}_{\widetilde{G}_{k}^{\triangleright} U_{k}}^{\widetilde{G}_{n}}\left(\gamma_{\psi^{\prime}} \otimes \psi\right)\right) \leq 1
$$

For $g \in G_{k}^{\triangle} U_{k} \backslash G_{n} / B_{n, *}$, denote

$$
\left.\mathcal{H}(g)=\operatorname{Hom}_{\left(\widetilde{G}_{k}^{\Delta} U_{k}\right)^{\mathfrak{s}(g)}}{ }^{(\mathfrak{s}(g)}\left(\gamma_{\psi^{\prime}}^{-1} \otimes \psi^{-1}\right) \otimes \chi, \delta\right),
$$

where $\left(\widetilde{G}_{k}^{\triangle} U_{k}\right)^{\mathfrak{s}(g)^{-1}}={ }^{\mathfrak{s}(g)^{-1}}\left(\widetilde{G}_{k}^{\triangle} U_{k}\right) \cap \widetilde{B}_{n, *} ;$ for a representation $\pi$ of $\widetilde{G}_{k}^{\triangle} U_{k},{ }^{\mathfrak{s}(g)} \pi$ is the representation of $\left(\widetilde{G}_{k}^{\triangle} U_{k}\right)^{\mathfrak{s}(g)^{-1}}$ on the space of $\pi$ given by ${ }^{\mathfrak{s}(g)} \pi(x)=\pi\left({ }^{\mathfrak{s}(g)} x\right) ; \delta(x)=\delta_{B_{n}}^{-1 / 2}(x) \delta_{g}(x)$ is a representation of $\left(G_{k}^{\triangle} U_{k}\right)^{g^{-1}}=g^{-1}\left(G_{k}^{\triangle} U_{k}\right) \cap B_{n, *}$ on $\mathbb{C}$, lifted to a nongenuine representation of the cover, where $\delta_{g}$ is the modulus character of $\left(G_{k}^{\triangle} U_{k}\right)^{g^{-1}}$. We recall that $\chi$ is a genuine character of $\widetilde{T}_{n, *}$ which is trivial on $\widetilde{T}_{n} \cap \kappa(K)$ (see the discussion following Remark 2.3). Also ${ }^{\mathfrak{s}(g)}\left(\gamma_{\psi^{\prime}}^{-1} \otimes \psi^{-1}\right) \otimes \chi$ acts trivially on $\mu_{2}$.

By [AG94] (proof of Proposition 1.3) $G_{n}=\bigcup_{w} G_{k}^{\triangle} U_{k} w B_{n}$ where $w$ varies over the permutation matrices. Hence we may assume $g=w t$ for a permutation matrix $w$ and $t \in T_{n}$. Set $\omega_{0}^{-1}=\left(\delta_{i, \tau(j)}\right)_{1 \leq i, j \leq k}, \omega^{-1}=\left({ }_{I_{k}} \omega_{0}^{-1}\right)$. 
By definition $\operatorname{dim} \mathcal{H}(g) \leq 1$ for any $g$. According to the Bruhat Theory (see e.g., Sil79, Theorems 1.9.4 and 1.9.5]), to prove (3.8) it is enough to show

$$
\begin{aligned}
& \mathcal{H}(g)=0, \quad \forall g=w t \text { such that } G_{k}^{\triangle} U_{k} w B_{n} \neq G_{k}^{\triangle} U_{k} \omega^{-1} B_{n}, \\
& \mathcal{H}\left(\omega^{-1} t\right)=0, \quad \forall t \text { such that } G_{k}^{\triangle} U_{k} \omega^{-1} t B_{n, *} \neq G_{k}^{\triangle} U_{k} \omega^{-1} B_{n, *} .
\end{aligned}
$$

Claim 3.11. Equality (3.9) holds.

Remark 3.12. In AG94] this claim already completed the proof of uniqueness, because in $G_{n}$ one could a priori take $g=w$.

Before proving the claim, let us complete the proof of the theorem. We establish (3.10). Put $g=\omega^{-1} t$. The condition on $t$ implies that we can assume $t=\operatorname{diag}\left(t_{1}, \ldots, t_{k}, I_{k}\right)$ and for some $1 \leq i \leq k, t_{i} \notin F^{* 2} \mathcal{O}^{*}$. Let $i$ be minimal with this property. Note that for any $y \in F^{*}-F^{* 2} \mathcal{O}^{*}$, there is $x \in \mathcal{O}^{*}$ such that $(y, x)_{2} \neq 1$. Then by (3.7) there is $x \in \mathcal{O}^{*}$ such that for $d=\operatorname{diag}\left(I_{i-1}, x, I_{k-i}\right)^{\triangle}$,

$$
\mathfrak{s}^{\mathfrak{s})^{-1}} \mathfrak{s}\left({ }^{\omega} d\right)=\left(t_{i}, x\right)_{2} \mathfrak{s}\left({ }^{\omega} d\right)=-\mathfrak{s}\left({ }^{\omega} d\right) .
$$

Then we obtain

$$
\mathfrak{s}(g)^{-1} \mathfrak{s}(d)={ }^{\mathfrak{s}(t)^{-1}}\left({ }^{\mathfrak{s}(\omega)} \mathfrak{s}(d)\right)={ }^{\mathfrak{s}(t)^{-1}}\left(\mathfrak{s}\left({ }^{\omega} d\right)\right)=-\mathfrak{s}\left({ }^{\omega} d\right) .
$$

Here for the second equality we used Corollary 3.9 and Claim 3.7. Clearly ${ }^{\mathfrak{s}(g)^{-1}} \mathfrak{s}(d) \in$ $\left(\widetilde{G}_{k}^{\triangle} U_{k}\right)^{\mathfrak{s}(g)^{-1}}$ and we see that $\left(\gamma_{\psi^{\prime}} \otimes \psi\right)(\mathfrak{s}(d))=1$ (because $\gamma_{\psi^{\prime}}$ is trivial on $\left.\mathcal{O}^{*}\right)$ and $\chi\left(^{\mathfrak{s}(g)^{-1}} \mathfrak{s}(d)\right)=$ $-\chi\left(\mathfrak{s}\left({ }^{\omega} d\right)\right)=-1$, because $\chi$ is trivial on $\mathfrak{s}\left(T_{n} \cap K\right)$. But $\delta_{B_{n}}$ and $\delta_{g}$ are modulus characters and as such, are trivial on $g^{-1} d$. Thus $\mathcal{H}\left(\omega^{-1} t\right)=0$.

Therefore the space of metaplectic Shalika functionals on $\mathrm{I}(\chi)$ is at most one-dimensional. For the second assertion of the theorem consider $\mathcal{H}\left(\omega^{-1}\right)$. In this case

$$
\left(G_{k}^{\triangle} U_{k}\right)^{\omega}=\iota\left(\left(T_{k} \cap K\right) T_{k}^{2}\right) \ltimes \prod_{\left\{\alpha \in \Sigma_{G_{k}}^{+}: \tau^{-1} \alpha>0\right\}} \iota\left(N_{\alpha}\right),
$$

where $N_{\alpha}$ on the right-hand side is a subgroup of $G_{k}$ and for any $c \in G_{k}, \iota(c)=\operatorname{diag}\left(c,{ }^{w_{0}} c\right)$. For $d=x^{\triangle}$ with $x \in T_{k}$ such that its coordinates lie in $F^{* 2} \mathcal{O}^{*},\left(\gamma_{\psi^{\prime}} \otimes \psi\right)(\mathfrak{s}(d))=1$. Also $\mathfrak{s}$ is a homomorphism of $\iota\left(\left(T_{k} \cap K\right) T_{k}^{2}\right)$. Hence

$$
\mathcal{H}\left(\omega^{-1}\right)=\operatorname{Hom}_{\mathfrak{s}\left(\iota\left(\left(T_{k} \cap K\right) T_{k}^{2}\right)\right)}\left(\chi, \delta_{B_{n}}^{-1 / 2} \delta_{\omega^{-1}}\right) .
$$

For all $1 \leq i \leq k, t_{i, k+\tau(i)} \in \mathfrak{s}\left(\iota\left(\left(T_{k} \cap K\right) T_{k}^{2}\right)\right)$ and a direct computation of $\delta_{\omega^{-1}}$, exactly as in the proof of Claim 3.11 below (only roots of the form $(l, i)$ with $1 \leq l<i$, and $(i, l)$ with $i<l \leq k$, may contribute), shows

$$
\delta_{\omega^{-1}}\left(t_{i, k+\tau(i)}\right)=|x|^{2(-|\{1 \leq l<i: \tau(l)<\tau(i)\}|+k-\tau(i)-|\{1 \leq l<i: \tau(l)>\tau(i)\}|)}=|x|^{2(k-\tau(i)-i+1)}=\delta_{B_{n}}^{1 / 2}\left(t_{i, k+\tau(i)}\right) .
$$

Hence there is no metaplectic Shalika functional on $\mathrm{I}(\chi)$, unless $\chi\left(t_{i, k+\tau(i)}\right)=1$.

Proof of Claim 3.11. As mentioned above, the arguments are similar to [AG94, Lemma 1.7]. One difference is that we consider a general $\tau$, whereas in AG94 the permutation was taken (for simplicity) to be the identity. This is only a minor complication.

Let $g=w t$ be as prescribed and write $w=\left(\delta_{i, j_{i}}\right)_{1 \leq i, j \leq n}$. For each $1 \leq l \leq k$, if $j_{l}<j_{k+l}$, then for $U=N_{(l, k+l)}$ (the root subgroup of $(l, k+l)$ ), ${ }^{w^{-1}} U=N_{j_{l}, j_{k+l}}<B_{n, *}$ and $\left.\psi\right|_{U} \neq 1$, and since $\left.\delta\right|_{U}=1, \mathcal{H}(g)=0$. Hence $j_{k+l}<j_{l}$ for all $1 \leq l \leq k$. 
We may assume $j_{\tau(1)}<\ldots<j_{\tau(k)}$. Suppose $j_{\tau(1)} \leq k$ and put $m=\tau(1)$. For $x \in F^{*}$ set

$$
d=\operatorname{diag}\left(I_{m-1}, x^{2}, I_{k-m}\right)^{\triangle} \in G_{k}^{\triangle} \cap B_{n, *} .
$$

Then $\mathfrak{s}^{\mathfrak{s}(g)^{-1}} \mathfrak{s}(d) \in\left(\widetilde{G}_{k}^{\triangle} U_{k}\right)^{\mathfrak{s}(g)^{-1}}$. Since $\mathfrak{s}(d) \in C_{\widetilde{T}_{n}}$, by Corollary 3.9

$$
\mathfrak{s}(g)^{-1} \mathfrak{s}(d)={ }^{\mathfrak{s}(w)^{-1}} \mathfrak{s}(d)=\mathfrak{s}\left(w^{-1} d\right)=t_{j_{k+m}, j_{m}} .
$$

On the one hand $\left(\gamma_{\psi^{\prime}} \otimes \psi\right)(\mathfrak{s}(d))=1$ and $\left.\chi \chi^{\left(\mathfrak{s}(g)^{-1}\right.} \mathfrak{s}(d)\right)=\chi\left(t_{j_{k+m}, j_{m}}\right) \neq 1$ by assumption (1) (because $\left.j_{k+m}<j_{m} \leq k\right)$. On the other hand, we show $\delta\left(g^{-1} d\right)=1$.

To compute $\delta\left(g^{-1} d\right)$, we count the roots from $U$ and $G_{k}^{\triangle}$ that are conjugated by $w$ to $N_{n}$. In the conjugation row $l$ moves into row $j_{l}$ and similarly for the columns. For the computation we only care about rows and columns $m$ and $k+m$. Our assumption on $\tau$ implies $\left\{l: j_{l}<j_{m}\right\} \subset\{l: l>k\}$.

First consider roots from $U$. Precisely $j_{m}-1$ roots from row $m$ are conjugated to columns smaller than $j_{m}$. The other $k-j_{m}+1$ roots are conjugated to columns bigger than $j_{m}$, so they do not contribute. Row $m+k$ and column $m$ do not contain any root of $U$, so do not contribute. Regarding column $m+k$, since $j_{m+k}<j_{m}$, the last $j_{m+k}-1$ rows from column $m+k$ are conjugated into the first $j_{m+k}-1$ rows of column $j_{m+k}$, and these do not contain roots. Summarizing, the roots from $U$ contribute $|x|^{2\left(k-j_{m}+1\right)}$.

Next consider $G_{k}^{\triangle}$. In general, a root $(i, j)$ with $1 \leq i \neq j \leq k$ contributes only if both the $(i, j)$-th and $(i+k, j+k)$-th coordinates are conjugated into $N_{n}$. The roots from row $m(k-1$ roots) are conjugated to columns greater than $j_{m}$. The last $j_{m}-1$ columns are conjugated to the first $j_{m}-1$ columns, so only $k-1-\left(j_{m}-1\right)$ roots from row $m$ contribute. Regarding row $m+k$, the first $k-j_{m}$ roots were already accounted for. But $j_{m}-j_{m+k}$ roots from the last $j_{m}$ columns are conjugated into columns $j_{m+k}+1, \ldots, j_{m}$, and also contribute because their copies from row $k$ were conjugated into positive roots. The rows conjugated into the first $j_{m}-1$ rows are from the last $k$ rows. Therefore, roots from column $m$ are not conjugated into positive roots in column $j_{m}$. Hence their copies in column $m+k$ cannot contribute as well. Altogether the roots from $G_{k}^{\triangle}$ contribute $|x|^{2\left(k-j_{m}\right)+2\left(j_{m}-j_{m+k}\right)}=|x|^{2\left(k-j_{m+k}\right)}$.

In total, $\delta\left(g^{-1} d\right)=|x|^{2 n-2\left(j_{m}+j_{k+m}-1\right)}=\delta_{B_{n}}^{1 / 2}\left(g^{-1} d\right)$, hence $\delta\left(g^{-1} d\right)=1$.

It follows that $\mathcal{H}(g)=0$, unless $j_{\tau(l)}>k$ for all $1 \leq l \leq k$. In the latter case we can assume $w=\left({ }_{I_{k}} w^{\prime}\right)$ (so, the assumption $j_{\tau(1)}<\ldots<j_{\tau(k)}$ is no longer valid). Let $1 \leq i_{1}, \ldots, i_{k} \leq k$ be such that $\tau\left(i_{l}\right)=l$ for all $1 \leq l \leq k$. We prove by induction on $l$, that $j_{i_{l}}=k+l$.

Suppose otherwise $j_{i}=k+1$ with $i \neq i_{1}$. Proceeding as above take

$$
d=\operatorname{diag}\left(I_{i-1}, x^{2}, I_{k-1}, x^{2}, I_{k-i}\right) \in G_{k}^{\triangle} \cap B_{n, *},
$$

then ${ }^{\mathfrak{s}(g)^{-1}} \mathfrak{s}(d)=\mathfrak{s}\left({ }^{w^{-1}} d\right)=t_{i, k+1}$. We see that $\left(\gamma_{\psi^{\prime}} \otimes \psi\right)(\mathfrak{s}(d))=1, \chi\left(\mathfrak{s}\left({ }^{w^{-1}} d\right)\right) \neq 1$ by assumption (21) (because $\tau(i) \neq \tau\left(i_{1}\right)=1$ ), while $\delta\left(g^{-1} d\right)=1$. In fact, we compute $\delta$ as above. Here only roots from $G_{k}^{\triangle}$ may contribute. On row $i$, the first $i-1$ roots do not contribute, because their copies are mapped into negative roots. On column $i$, the positive roots do not contribute because they are mapped into rows greater than $k+1$ of column $k+1$. This leaves the last $k-i$ roots of row $i$, which are mapped into row $k+1$ and columns greaater than $k+1$, and their copies are mapped into positive roots. Altogether $\delta_{g}\left(g^{-1} d\right)=|x|^{2(k-i)}=\delta_{B_{n}}^{1 / 2}\left(g^{-1} d\right)$. Thus $\mathcal{H}(g)=0$, unless $j_{i_{1}}=k+1$.

Assuming for all $1 \leq l^{\prime}<l, j_{i_{l^{\prime}}}=k+l^{\prime}$, we show $j_{i_{l}}=k+l$. Suppose $j_{i}=k+l$ for $i \neq i_{l}$. Then $i \neq i_{l^{\prime}}$ for $1 \leq l^{\prime} \leq l$. We take $d$ as in (3.11). Then ${ }^{\mathfrak{s}(g)^{-1}} \mathfrak{s}(d)=t_{i, k+l}, \chi\left(\mathfrak{s}\left(w^{-1} d\right)\right) \neq 1$ 
and we have to show $\delta\left(g^{-1} d\right)=1$. Indeed, as in the case $l=1$, we need only consider the positive roots from $G_{k}^{\triangle}$, on the $i$-th row and column. Their copies are conjugated to positive roots. According to the induction hypothesis, for each $1 \leq l^{\prime}<l$, the $\left(i_{l^{\prime}}, i\right)$-th (resp. $\left(i, i_{l^{\prime}}\right)$ th) entry is conjugated into a positive root if and only if $i_{l^{\prime}}<i$ (resp. $i_{l^{\prime}}>i$ ). This implies that the contribution consists of $\left|\left\{1 \leq l^{\prime}<l: i_{l^{\prime}}<i\right\}\right|$ roots from column $i$ (contributing negative powers of $|x|$ ) and $k-i-\left|\left\{1 \leq l^{\prime}<l: i_{l^{\prime}}>i\right\}\right|$ from row $i$ (contributing positive powers). Thus $\delta_{g}\left(g^{-1} d\right)=|x|^{2(k-i-l+1)}=\delta_{B_{n}}^{1 / 2}\left(g^{-1} d\right)$ and $\mathcal{H}(g)=0$, unless $j_{i_{l}}=k+l$. The proof is complete, since $j_{l}=k+\tau(l)$ for all $1 \leq l \leq k$, i.e., $w^{\prime}=\omega_{0}^{-1}$ and $w=\omega^{-1}$.

Remark 3.13. Recall that $\chi=\chi_{\underline{s}}, \underline{s} \in \mathbb{C}^{n}$ (see $\S$ [2.2). Assume $q^{2 s_{i}} \neq q^{ \pm 2 s_{j}}$ for all $1 \leq$ $i<j \leq k$ and $q^{2 s_{i}}=q^{-2 s_{k+i}}$ for all $1 \leq i \leq k$, perhaps after a reordering of $\left(s_{k+1}, \ldots, s_{n}\right)$. By Theorem 3.10, $\mathrm{I}(\chi)$ and $\mathrm{I}\left(\chi^{-1}\right)$ admit at most one metaplectic Shalika functional (up to scalar multiplication).

Remark 3.14. If $\mathrm{I}(\chi)$ is irreducible, then we may have simply fixed one permutation $\tau$ in the statement of the theorem. However, we will also apply the theorem to reducible representations.

We define a functional satisfying the prescribed equivariance properties (3.2). It will be defined using an integral, which is absolutely convergent in a certain cone in $\chi$. Then we extend it to a meromorphic function, thereby obtaining a functional for all $\chi$.

Following Sakellaridis [Sak06], we realize the metaplectic Shalika functional using a similar functional on a group $H$, which is a twist of the Shalika group. Working with $H$ instead of the Shalika group was advantageous in [Sak06] for computational reasons. Let $J_{k}$ be the permutation matrix

$$
J_{k}=\left(\begin{array}{ccc}
0 & & 1 \\
& . & \\
1 & & 0
\end{array}\right) \in G_{k}
$$

Put

$$
H={ }^{\omega}\left(G_{k}^{\triangle} U_{k}\right)=\left\{\left(\begin{array}{cc}
c & 0 \\
J_{k} c u & J_{k} c J_{k}
\end{array}\right): c \in G_{k}\right\}, \quad \omega=\left(\begin{array}{cc} 
& I_{k} \\
J_{k} &
\end{array}\right) .
$$

We form a section $\mathfrak{h}$ of $H$ by

$$
\mathfrak{h}(h)=\mathfrak{s}(\omega) \mathfrak{s}\left(\omega^{-1} h\right) \mathfrak{s}(\omega)^{-1} .
$$

For $h \in H$, write $\omega^{-1} h=c_{h}^{\triangle} u_{h}$ with $c_{h} \in G_{k}$ and $u_{h} \in U_{k}$. The mapping $h \mapsto c_{h}$ is a group epimorphism, the mapping $h \mapsto u_{h}$ is onto $U$, and $h \mapsto \psi\left(u_{h}\right)$ is a character of $H$ (trivial on $\left.{ }^{\omega} G_{k}^{\triangle}\right)$. According to (3.1), the section $\mathfrak{h}$ satisfies

$$
\mathfrak{h}\left(h h^{\prime}\right)=\left(\operatorname{det} c_{h}, \operatorname{det} c_{h^{\prime}}\right)_{2} \mathfrak{h}(h) \mathfrak{h}\left(h^{\prime}\right) .
$$

A functional $l$ on a genuine $\pi \in \operatorname{Alg} \widetilde{G}_{n}$ is called a (metaplectic) $\left(H, \psi^{\prime}, \psi\right)$-functional if for any $\xi$ in the space of $\pi$ and $h \in H$,

$$
l(\pi(\mathfrak{h}(h)) \xi)=\gamma_{\psi^{\prime}}\left(\operatorname{det} c_{h}\right) \psi\left(u_{h}\right) l(\xi) .
$$

The definitions of $H$ and $\mathfrak{h}$ imply the following relation between these functionals and metaplectic Shalika functionals. 
Claim 3.15. Let $\pi \in \operatorname{Alg} \widetilde{G}_{n}$ be genuine. The dimension of the space of $\left(H, \psi^{\prime}, \psi\right)$-functionals on $\pi$ is equal to the dimension of metaplectic $\left(\psi^{\prime}, \psi\right)$-Shalika functionals.

Proof. If $l$ is an $\left(H, \psi^{\prime}, \psi\right)$-functional on $\pi, \xi \mapsto l(\pi(\mathfrak{s}(\omega)) \xi)$ is a $\left(\psi^{\prime}, \psi\right)$-Shalika functional. If $l$ is a $\left(\psi^{\prime}, \psi\right)$-Shalika functional, $\xi \mapsto l\left(\pi\left(\mathfrak{s}(\omega)^{-1}\right) \xi\right)$ is an $\left(H, \psi^{\prime}, \psi\right)$-functional.

With Claim 3.15 in mind, we construct $\left(H, \psi^{\prime}, \psi\right)$-functionals. Our main tool will be the metaplectic analog of Hironaka's theorem, Corollary 2.17. To preserve the notation, we construct the functional on $\mathrm{I}\left(\chi^{-1}\right)$.

The group $H$ is closed and unimodular. Sakellaridis proved [Sak06, Lemma 3.1] that $B_{n} H$ is Zariski open in $G_{n}$. Therefore $B_{n} H$ is dense and open in $G_{n}$, making it convenient for integration formulas. Here we are forced to work with $B_{n, *} H$, which is in particular not dense. Next we describe certain structural results involving $B_{n, *}$ and $H$, which will enable us to adapt the integration formulas from [Sak06] to the metaplectic setting.

Claim 3.16. $B_{n} H=\coprod_{t \in T_{k, *} \backslash T_{k}} \operatorname{diag}\left(t, I_{k}\right) B_{n, *} H$.

Proof. Since $H$ contains the subgroup $\left\{\operatorname{diag}\left(x_{1}, \ldots, x_{k}, x_{k}, \ldots, x_{1}\right): x_{i} \in F^{*}\right\}$, it is clear that any $b h \in B_{n} H$ is contained in one of the cosets on the right-hand side. The union is disjoint because if $\operatorname{diag}\left(t, I_{k}\right) B_{n, *} H=\operatorname{diag}\left(t^{\prime}, I_{k}\right) B_{n, *} H$, using the fact that $B_{n, *}$ is normal in $B_{n}$ leads to $\operatorname{diag}\left(t t^{\prime-1}, I_{k}\right) b_{*} \in H$ for some $b_{*} \in B_{n, *}$. Let the diagonal of $b_{*}$ be $\operatorname{diag}\left(c_{1}, \ldots, c_{n}\right)$, $c_{i} \in F^{* 2} \mathcal{O}^{*}$. Then the diagonal of $\operatorname{diag}\left(t t^{\prime-1}, I_{k}\right) b_{*}$ takes the form $\operatorname{diag}\left(x_{1}, \ldots, x_{k}, x_{k}, \ldots, x_{1}\right)$ and we must have $t_{i} t_{i}^{\prime-1} c_{i}=x_{i}=c_{n-i+1}$ for all $1 \leq i \leq k$, whence $t_{i} t_{i}^{\prime-1} \in F^{* 2} \mathcal{O}^{*}$ for all $i$ and $t T_{k, *}=t^{\prime} T_{k, *}$.

We will use the following geometric results from [Sak06, Lemma 5.1]:

$$
\begin{aligned}
& N_{\alpha}^{-}(\varpi \mathcal{O}) \subset\left(T_{n} \cap K\right) N_{\alpha}(\mathcal{O}) N_{\alpha^{\prime}}(\mathcal{O})(H \cap K), \quad \forall \alpha>0, \\
& \mathcal{I}^{-} \subset\left(B_{n} \cap K\right)(H \cap K) .
\end{aligned}
$$

Here for $(i, j)=\alpha>0, \alpha^{\prime}=\alpha$ if $i \leq k<j$ otherwise $\alpha^{\prime}=(n-j+1, n-i+1)$. The latter inclusion along with (2.1) and the fact that $B_{n} \cap K<B_{n, *}$ implies

$$
\mathcal{I} \subset\left(B_{n} \cap K\right)(H \cap K) \subset B_{n, *} H .
$$

Claim 3.17. The set $B_{n, *} H$ is open $\left(\right.$ in $\left.G_{n}\right)$.

Proof. According to (3.15), for any $b_{*} \in B_{n, *}$ and $h \in H, b_{*} \mathcal{I} h \subset B_{n, *} H$. The set $b_{*} \mathcal{I} h$ is open (because $\mathcal{I}$ is) and contains $b_{*} h$. Therefore $B_{n, *} H$ is open.

The following two claims relate the sections $\mathfrak{h}, \mathfrak{s}$ and $\kappa$.

Claim 3.18. The sections $\mathfrak{s}$ and $\kappa$ agree on $\left(G_{k}^{\triangle} U_{k}\right) \cap K$. Consequently, $\mathfrak{h}$ and $\kappa$ agree on $H \cap K$.

Proof. First we compare $\mathfrak{s}$ and $\kappa$. We have $\left(G_{k}^{\triangle} U_{k}\right) \cap K=G_{k}(\mathcal{O})^{\triangle} U_{k}(\mathcal{O})$, where $G_{k}(\mathcal{O})^{\triangle}=$ $\left\{c^{\triangle}: c \in G_{k}(\mathcal{O})\right\}$. According to (3.1) and because $\left(\mathcal{O}^{*}, \mathcal{O}^{*}\right)_{2}=1, \mathfrak{s}$ is a splitting of $G_{k}(\mathcal{O})^{\triangle}$ and thereby of $G_{k}(\mathcal{O})^{\triangle} U_{k}(\mathcal{O})$. The section $\kappa$ is also a splitting of the latter. Since $\left.\mathfrak{s}\right|_{N_{n} \cap K}=\left.\kappa\right|_{N_{n} \cap K}$, it is enough to show that $\mathfrak{s}$ and $\kappa$ agree on $G_{k}(\mathcal{O})^{\triangle}$. The mapping $c \mapsto \mathfrak{s}\left(c^{\triangle}\right) \kappa\left(c^{\triangle}\right)^{-1}$ is a homomorphism $G_{k}(\mathcal{O}) \rightarrow \mu_{2}$, which is trivial on $T_{k} \cap G_{k}(\mathcal{O})$ because $\left.\mathfrak{s}\right|_{T_{n} \cap K}=\left.\kappa\right|_{T_{n} \cap K}$. Moreover, there is only one homomorphism $\mathrm{SL}_{k}(\mathcal{O}) \rightarrow \mu_{2}$, the trivial one (when $|2|=1$ and $q>3$ ). Thus $c \mapsto \mathfrak{s}\left(c^{\triangle}\right) \kappa\left(c^{\triangle}\right)^{-1}$ is the identity. 
Next we have $H \cap K={ }^{\omega}\left(G_{k}(\mathcal{O})^{\triangle} U_{k}(\mathcal{O})\right)$. The section $\mathfrak{h}$ is a splitting of $H \cap K$ by definition and because $\mathfrak{s}$ is a splitting of $\left(G_{k}^{\triangle} U_{k}\right) \cap K$. The assertion for $\mathfrak{h}$ now follows from the previous result because $\kappa(\omega)=\mathfrak{s}(\omega)$.

Remark 3.19. One can check that for $u \neq 0, \mathfrak{h}\left(\begin{array}{ll}1 & 0 \\ u & 1\end{array}\right)=(u, u)_{2} \mathfrak{s}\left(\begin{array}{ll}1 & 0 \\ u & 1\end{array}\right)$, whence $\mathfrak{h}$ and $\mathfrak{s}$ do not coincide, even on ${ }^{\omega} U_{k}(\mathcal{O})$.

Next we consider

$$
H \cap B_{n, *}=\left\{\operatorname{diag}\left(t_{1}, \ldots, t_{k}, t_{k}, \ldots, t_{1}\right): t_{i} \in F^{* 2} \mathcal{O}^{*}\right\} .
$$

It is closed and unimodular.

Claim 3.20. The sections $\mathfrak{h}$ and $\mathfrak{s}$ agree on $H \cap B_{n, *}$.

Proof. Let $t=\operatorname{diag}\left(t_{1}, \ldots, t_{k}, t_{k}, \ldots, t_{1}\right) \in H \cap B_{n, *}$. Then $\mathfrak{h}(t)={ }^{\mathfrak{s}(\omega)} \mathfrak{s}\left(\omega^{-1} t\right)$. We compute this conjugation as explained in Remark 3.8. Put $\omega=t_{0} \mathfrak{w}$, where $t_{0} \in T_{n} \cap K$ and $\mathfrak{w} \in \mathfrak{W}$. Then $\sigma(\mathfrak{w}, t)=1$ because $(\cdot, \cdot)_{2}$ is trivial on $F^{* 2} \mathcal{O}^{*} \times F^{* 2} \mathcal{O}^{*}$. Also $\mathfrak{s}\left(t_{0}\right)$ and $\mathfrak{s}(t)$ commute because $t_{0}, t \in T_{n, *}$. Thus ${ }^{\mathfrak{s}(\omega)} \mathfrak{s}\left(\omega^{-1} t\right)=\mathfrak{s}(t)$.

Let $d h$ be a Haar measure on $H$. Consider the functional $l_{H}$ on $\mathcal{S}^{\text {gen }}(\widetilde{H})$ given by

$$
l_{H}(f)=\int_{H} f(\mathfrak{h}(h)) \gamma_{\psi^{\prime}}^{-1}\left(\operatorname{det} c_{h}\right) \psi^{-1}\left(u_{h}\right) d h .
$$

This integral is absolutely convergent because $|f| \in \mathcal{S}^{\text {gen }}(H)(|f|$ is non-genuine and $p: \widetilde{H} \rightarrow$ $H$ is continuous). Since $f$ is genuine, (3.12) and (1.17) imply

$$
l_{H}(\mathfrak{h}(h) f)=\gamma_{\psi^{\prime}}\left(\operatorname{det} c_{h}\right) \psi\left(u_{h}\right) l_{H}(f) .
$$

Hence $l_{H}$ satisfies (3.13) and is an $\left(H, \psi^{\prime}, \psi\right)$-functional on the space of $\mathcal{S}^{\text {gen }}(\widetilde{H})$, where $\widetilde{H}$ acts by right-translations.

Next consider the space of $\mathcal{S}^{\text {gen }}\left(\mathfrak{s}\left(H \cap B_{n, *}\right) \backslash \widetilde{H}\right)$. There is a surjection

$$
\mathcal{S}^{\text {gen }}\left(p^{-1}\left(B_{n, *} H\right)\right) \rightarrow \mathcal{S}^{\text {gen }}\left(\mathfrak{s}\left(H \cap B_{n, *}\right) \backslash \widetilde{H}\right)
$$

given by $f \mapsto f^{B_{n, *} \cap H}$, where

$$
f^{B_{n, *} \cap H}(h)=\int_{B_{n, *} \cap H} f\left(\mathfrak{s}\left(b_{*}\right) h\right) d b_{*}, \quad h \in \widetilde{H} .
$$

Note that $\mathfrak{h}\left(b_{*}\right)=\mathfrak{s}\left(b_{*}\right)$ (Claim 3.20$)$. Then we have a functional $l_{H \cap B_{n, *} \backslash H}$ on $\mathcal{S}^{\text {gen }}(\mathfrak{s}(H \cap$ $\left.\left.B_{n, *}\right) \backslash \widetilde{H}\right)$ defined by (see [Sak06, (44)])

$$
l_{H \cap B_{n, *} \backslash H}(f)=\int_{H \cap B_{n, *} \backslash H} f(\mathfrak{h}(h)) \gamma_{\psi^{\prime}}^{-1}\left(\operatorname{det} c_{h}\right) \psi^{-1}\left(u_{h}\right) d h .
$$

The integrand is invariant on the left by $H \cap B_{n, *}$ : for $b_{*} \in H \cap B_{n, *}$ and $h \in H$, by (3.12), Claim 3.20, (1.17) and the fact that $h \mapsto \gamma_{\psi^{\prime}}^{-1}\left(\operatorname{det} c_{h}\right)$ is trivial on $H \cap B_{n, *}$,

$$
\begin{aligned}
\gamma_{\psi^{\prime}}^{-1}\left(\operatorname{det} c_{b_{*} h}\right) \mathfrak{h}\left(b_{*} h\right) & =\gamma_{\psi^{\prime}}^{-1}\left(\operatorname{det} c_{b_{*}} \operatorname{det} c_{h}\right)\left(\operatorname{det} c_{b_{*}}, \operatorname{det} c_{h}\right)_{2} \mathfrak{h}\left(b_{*}\right) \mathfrak{h}(h) \\
& =\gamma_{\psi^{\prime}}^{-1}\left(\operatorname{det} c_{b_{*}}\right) \gamma_{\psi^{\prime}}^{-1}\left(\operatorname{det} c_{h}\right) \mathfrak{s}\left(b_{*}\right) \mathfrak{h}(h) \\
& =\gamma_{\psi^{\prime}}^{-1}\left(\operatorname{det} c_{h}\right) \mathfrak{s}\left(b_{*}\right) \mathfrak{h}(h) .
\end{aligned}
$$


Hence

$$
f\left(\mathfrak{h}\left(b_{*} h\right)\right) \gamma_{\psi^{\prime}}^{-1}\left(\operatorname{det} c_{b_{*} h}\right)=f(\mathfrak{h}(h)) \gamma_{\psi^{\prime}}^{-1}\left(\operatorname{det} c_{h}\right) .
$$

Also the character $\psi^{-1}$ satisfies $\psi^{-1}\left(u_{b_{*} h}\right)=\psi^{-1}\left(u_{h}\right)$ because $u_{b_{*}}=I_{n}$. Of course, $l_{H \cap B_{n, *} \backslash H}$ satisfies the same equivariance properties as $l_{H}$.

Henceforth assume

$$
q^{2 s_{i}} \neq q^{ \pm 2 s_{j}}, \quad \forall 1 \leq i<j \leq k, \quad q^{2 s_{i}}=q^{-2 s_{n-i+1}}, \quad \forall 1 \leq i \leq k .
$$

By Theorem 3.10 and Claim 3.15 the space of $\left(H, \psi^{\prime}, \psi\right)$-functionals on $\mathrm{I}\left(\chi^{-1}\right)$ is at most one-dimensional.

We chose $\chi$ in this form so that it is trivial on $\mathfrak{s}\left(H \cap B_{n, *}\right)$. The modulus character $\delta_{B_{n}}$ is also trivial on $H \cap B_{n, *}$. We may then regard the elements of $\mathrm{I}\left(\chi^{-1}\right)$ as genuine locally constant functions on $\mathfrak{s}\left(H \cap B_{n, *}\right) \backslash \widetilde{H}$. Define a functional $\Lambda_{\chi}$ on $\mathrm{I}\left(\chi^{-1}\right)$ by

$$
\Lambda_{\chi}\left(f_{\chi^{-1}}\right)=l_{H \cap B_{n, *} \backslash H}\left(f_{\chi^{-1}}\right) .
$$

First we prove that this makes sense, for sufficiently many characters $\chi$. Later we extend it to all relevant characters by meromorphic continuation. The equivariance properties of $l_{H}$ imply that $\Lambda_{\chi}$ is an $\left(H, \psi^{\prime}, \psi\right)$-functional.

Claim 3.21. Assuming $\operatorname{Re}\left(s_{i}\right)<\operatorname{Re}\left(s_{i+1}\right)$ for all $1 \leq i<k$ and $\operatorname{Re}\left(s_{k}\right)<0$, the integral $l_{H \cap B_{n, *} \backslash H}\left(f_{\chi^{-1}}\right)$ given by (3.16) is absolutely convergent for all $f_{\chi^{-1}}$.

Proof. Since $\left|f_{\chi^{-1}}\right|$ can be regarded as a function on $G_{n}$, the proof in [Sak06, Proposition 7.1] applies to our setting as well. Briefly, writing $d h$ on $A_{k} \backslash T_{k} \times N_{k} \times G_{k}(\mathcal{O})$ with $A_{k}=$ $\left(T_{k} \cap G_{k}(\mathcal{O})\right) T_{k}^{2}$,

$$
\int_{H \cap B_{n, *} \backslash H}\left|f_{\chi^{-1}}\right|(h) d h=\int_{U_{k}} \sum_{t \in A_{k} \backslash T_{k}} \int_{N_{k}} \int_{G_{k}(\mathcal{O})}\left|f_{\chi^{-1}}\right|\left(\omega t^{\triangle} v^{\triangle} k^{\triangle} u \omega^{-1}\right) d k d v d u .
$$

We may assume $f_{\chi^{-1}}$ is unramified. Then the integral is majorized by a finite sum of integrals

$$
\int_{U_{k}} \int_{N_{k}}\left|f_{\chi^{-1}}\right|\left(\omega\left(\begin{array}{cc}
v & u \\
& I_{k}
\end{array}\right) \omega^{-1 \omega}\left(t^{\triangle}\right)\right) d v d u
$$

(we omitted a constant depending on the change of measure $d v d u$, caused by the conjugation by $t^{\triangle}$ ), which are known to converge given our assumption on $\chi$.

Recall the surjection $P_{\chi}: \mathcal{S}^{\text {gen }}\left(\widetilde{G}_{n}\right) \rightarrow \mathrm{I}(\chi)$ given by (2.3). We combine $P_{\chi^{-1}}$ with (3.16) to obtain a formula convenient for computations (as in [Sak06, (45)]). For $g \in B_{n, *} H$, one can write $g=b_{*} h$ with $b_{*} \in B_{n, *}$ and $h \in H$. This writing is not unique. We still put $b_{g}=b_{*}$ and $h_{g}=h$. The arguments after (3.16) imply that the mappings $g \mapsto \psi^{-1}\left(u_{h_{g}}\right)$ and $g \mapsto \mathfrak{s}\left(b_{g}\right) \mathfrak{h}\left(h_{g}\right) \gamma_{\psi^{\prime}}^{-1}\left(\operatorname{det} c_{h_{g}}\right)$ are well defined. Also $g \mapsto \delta_{B_{n}}^{1 / 2}\left(b_{g}\right)$ is independent of the choice of $b_{g}$ and the assumption $\left.\chi\right|_{\mathfrak{s}\left(H \cap B_{n, *}\right)}=1$ implies $g \mapsto \chi\left(\mathfrak{s}\left(b_{g}\right)\right)$ is independent of the specific writing. Now we formally obtain

$$
\Lambda_{\chi}\left(P_{\chi^{-1}}(f)\right)=\int_{B_{n, *} H} \delta_{B_{n}}^{1 / 2}\left(b_{g}\right) \chi\left(\mathfrak{s}\left(b_{g}\right)\right) f\left(\mathfrak{s}\left(b_{g}\right) \mathfrak{h}\left(h_{g}\right)\right) \gamma_{\psi^{\prime}}^{-1}\left(\operatorname{det} c_{h_{g}}\right) \psi^{-1}\left(u_{h_{g}}\right) d g .
$$

This formula is valid whenever the right-hand side is absolutely convergent. The measure $d g$ is taken to be the restriction of the Haar measure on $G_{n}$ to $B_{n, *} H$ and on the left-hand side 
we normalize the measure $d h$ accordingly (the measure $d_{l} b_{*}$ has already been normalized, see after (2.3) $)$. Let

For any $\lambda=\left(\lambda_{1}, \ldots, \lambda_{k}\right) \in \mathbb{Z}^{k}$, put $\varpi^{\lambda}=\operatorname{diag}\left(\varpi^{\lambda_{1}}, \ldots, \varpi^{\lambda_{k}}\right)$ and denote $t_{\lambda}=\operatorname{diag}\left(\varpi^{\lambda}, I_{k}\right)$.

$$
\begin{aligned}
& \mathbb{Z}_{\geq}^{k}=\left\{\left(\lambda_{1}, \ldots, \lambda_{k}\right) \in \mathbb{Z}^{k}: \lambda_{1} \geq \ldots \geq \lambda_{k}\right\}, \\
& \mathbb{Z}_{+}^{k}=\left\{\left(\lambda_{1}, \ldots, \lambda_{k}\right) \in \mathbb{Z}^{k}: \lambda_{1} \geq \ldots \geq \lambda_{k} \geq 0\right\} .
\end{aligned}
$$

We also use $2 \mathbb{Z}_{+}^{k}$, where for all $i, \lambda_{i} \in 2 \mathbb{Z}$.

The following lemma describes the evaluation of $\Lambda_{\chi}\left(\varphi_{w, \chi^{-1}}\right)$ for certain elements $w$, where it can be done succinctly. It is the main technical result of this section. The proof is parallel to the proof of Propositions 5.2, 8.1 and 8.2 in [Sak06], the complications involve the various sections $\mathfrak{s}, \mathfrak{h}$ and $\kappa$. The results will be used below to deduce the general formula.

Lemma 3.22. Assume $\operatorname{Re}\left(s_{i}\right)<\operatorname{Re}\left(s_{i+1}\right)$ for all $1 \leq i<k$ and $\operatorname{Re}\left(s_{k}\right)<0$. Also assume $\psi^{\prime}=\psi_{\varrho}$, for some $\varrho \in \mathcal{O}^{*}$ (i.e., $\psi^{\prime}(x)=\psi(\varrho x)$ ). The following holds.

$$
\begin{gathered}
\Lambda_{\chi}\left(\mathfrak{s}\left(t_{-\lambda}\right) \varphi_{e, \chi^{-1}}\right)= \begin{cases}\operatorname{vol}(\mathcal{I}) \delta_{B_{n}}^{1 / 2} \chi\left(\mathfrak{s}\left(t_{\lambda}\right)\right) & \lambda \in 2 \mathbb{Z}_{+}^{k}, \\
0 & \lambda \in \mathbb{Z}_{+}^{k}-2 \mathbb{Z}_{+}^{k} .\end{cases} \\
\Lambda_{\chi}\left(\varphi_{s_{\alpha_{k}}, \chi^{-1}}\right)=\operatorname{vol}(\mathcal{I} \mathfrak{w} \mathcal{I})\left(1-q^{-1}+(-\varrho, \varpi)_{2}(\varpi, \varpi)_{2}^{k-1} q^{-1 / 2} \chi^{-1 / 2}\left(a_{\alpha_{k}}\right)\right) .
\end{gathered}
$$

For all $1 \leq i<k$,

$$
\Lambda_{\chi}\left(\varphi_{s_{\alpha_{i}} s_{\alpha_{n-i}}, \chi^{-1}}\right)=\operatorname{vol}(\mathcal{I} \mathfrak{w} \mathcal{I})\left(1-q^{-1}+q^{-2}+\left(1-q^{-1}\right)^{2} \frac{\chi^{-1}\left(a_{\alpha_{i}}\right)}{1-\chi^{-1}\left(a_{\alpha_{i}}\right)}\right) .
$$

Here $\chi^{-1 / 2}\left(a_{\alpha_{k}}\right)=q^{2 s_{k}}$.

Remark 3.23. Note that $\chi^{-1 / 2}\left(a_{\alpha}\right)$ is well defined because $\chi\left(\mathfrak{s}\left(\operatorname{diag}\left(I_{i-1}, \varpi^{2}, I_{n-i}\right)\right)\right)=q^{-2 s_{i}}$.

Proof. We use (3.18) for the computation. We first proceed formally, the computation will also imply that (3.18) is absolutely convergent, providing the justification for replacing (3.16) with (3.18).

Consider (3.19) and assume $\lambda \in \mathbb{Z}_{+}^{k}$. Note that $t_{-\lambda}^{-1}=t_{\lambda}$. The integrand vanishes unless $g \in \mathcal{I} t_{\lambda}$. For $\lambda \in \mathbb{Z}_{+}^{k}, t_{\lambda} \in T_{n}^{-}$whence $t_{\lambda}^{-1}\left(\mathcal{I}^{-}\right) t_{\lambda}<\mathcal{I}^{-}$and by (2.1) and (3.15),

$$
\mathcal{I} t_{\lambda} \subset\left(B_{n} \cap K\right) t_{\lambda}\left(B_{n} \cap K\right)(H \cap K) .
$$

In particular $\mathcal{I} t_{\lambda} \subset t_{\lambda} B_{n, *} H$, the latter does not intersect $B_{n, *} H$ unless $\lambda \in 2 \mathbb{Z}_{+}^{k}$, by Claim 3.16. So the integrand vanishes unless $\lambda \in 2 \mathbb{Z}_{+}^{k}$. In this case one can take $b_{g}=b_{0} t_{\lambda} b_{0}^{\prime}$ for some $b_{0}, b_{0}^{\prime} \in B_{n} \cap K$ and $h_{g} \in H \cap K$. Then $\psi\left(u_{h_{g}}\right)=\gamma_{\psi^{\prime}}^{-1}\left(\operatorname{det} c_{h_{g}}\right)=1$ because $h_{g} \in K$, so that both (3.18) and (3.16) equal

$$
\operatorname{vol}(\mathcal{I}) \delta_{B_{n}}^{1 / 2} \chi\left(\mathfrak{s}\left(t_{\lambda}\right)\right)
$$

Next we prove (3.20). Let $\mathfrak{w}$ be the representative of $s_{\alpha_{k}}$. Using (2.1) and (3.14) we obtain

$$
\mathcal{I} \mathfrak{w} \mathcal{I}=\left(B_{n} \cap K\right) \mathfrak{w} N_{\alpha_{k}}(\mathcal{O}) \prod_{\beta>0: \beta \neq \alpha_{k}} N_{\beta}^{-}(\varpi \mathcal{O}) \subset\left(B_{n} \cap K\right) \mathfrak{w} N_{\alpha_{k}}(\mathcal{O})(H \cap K) .
$$

The integrand vanishes unless $g \in \mathcal{I} \mathfrak{w} \mathcal{I}$. Write

$$
g=b_{0} \mathfrak{w} v h_{0}, \quad b_{0} \in B_{n} \cap K, \quad v \in N_{\alpha_{k}}(\mathcal{O}), \quad h_{0} \in H \cap K .
$$


We decompose $\mathfrak{w} v=b_{*} h \in B_{n, *} H$, using $2 \times 2$ matrix computations, where the matrices are regarded as elements of $G_{2}$ embedded in $G_{n}$ such that its derived group is $\mathcal{R}_{\alpha_{k}}\left(\mathcal{R}_{\alpha_{k}}\right.$ was defined after (2.5)). Then $\mathfrak{w}$ is the matrix $\left({ }_{1}{ }^{-1}\right)$. If $v=\left(\begin{array}{cc}1 & z \\ 1\end{array}\right)$ and $z \neq 0$,

$$
b_{*}=\left(\begin{array}{cc}
1 & -z \\
& z^{2}
\end{array}\right), \quad h=\left(\begin{array}{ll}
z^{-1} & \\
z^{-2} & z^{-1}
\end{array}\right) .
$$

Then $b_{g}=b_{0} b_{*}$ and $h_{g}=h h_{0}$, hence

$$
\begin{aligned}
& \psi^{-1}\left(u_{h_{g}}\right)=\psi^{-1}\left(u_{h}\right)=\psi^{-1}\left(z^{-1}\right) \\
& \gamma_{\psi^{\prime}}^{-1}\left(\operatorname{det} c_{h_{g}}\right)=\left(\operatorname{det} c_{h}, \operatorname{det} c_{h_{0}}\right)_{2} \gamma_{\psi^{\prime}}^{-1}\left(\operatorname{det} c_{h}\right)=\left(z, \operatorname{det} c_{h_{0}}\right)_{2} \gamma_{\psi^{\prime}}^{-1}\left(z^{-1}\right), \\
& \delta_{B_{n}}^{1 / 2}\left(b_{g}\right)=|z|^{-1} \\
& \chi\left(\mathfrak{s}\left(b_{g}\right)\right)=|z|^{2 s_{k+1}}=|z|^{-2 s_{k}} .
\end{aligned}
$$

Next we show

$$
\operatorname{ch}_{s_{\alpha_{k}}, \chi^{-1}}\left(\mathfrak{s}\left(b_{g}\right) \mathfrak{h}\left(h_{g}\right)\right)=(z, z)_{2}^{k-1}\left(z, \operatorname{det} c_{h_{0}}\right)_{2} .
$$

Because $g \in \mathcal{I} \mathfrak{w} \mathcal{I}$, this will follow immediately from

$$
\mathfrak{s}\left(b_{g}\right) \mathfrak{h}\left(h_{g}\right)=(z, z)_{2}^{k-1}\left(z, \operatorname{det} c_{h_{0}}\right)_{2} \kappa(g) .
$$

To show this, start with $\kappa(g)=\kappa\left(b_{0}\right) \kappa(\mathfrak{w}) \kappa(v) \kappa\left(h_{0}\right)$. Since $\mathfrak{s}(\mathfrak{w}) \mathfrak{s}(v)=\mathfrak{s}(\mathfrak{w} v)=\mathfrak{s}\left(b_{*} h\right)$,

$$
\kappa(g)=\sigma\left(b_{*}, h\right) \kappa\left(b_{0}\right) \mathfrak{s}\left(b_{*}\right) \mathfrak{s}(h) \kappa\left(h_{0}\right) .
$$

Using (1.2) we see $\sigma\left(b_{*}, h\right)=1$. Next we claim $\mathfrak{s}(h)=(z, z)_{2}^{k-1} \mathfrak{h}(h)$. Start with $\mathfrak{h}(h)=$ $\mathfrak{s}(\omega) \mathfrak{s}\left(\omega^{-1} h\right) \mathfrak{s}(\omega)^{-1}$. Write ${ }^{\omega^{-1}} h=c u$, where $c=\operatorname{diag}\left(I_{k-1}, z^{-1}, I_{k-1}, z^{-1}\right)$ and

$$
u=\left(\begin{array}{cccc}
I_{k-1} & & & \\
& 1 & & z^{-1} \\
& & I_{k-1} & \\
& & & 1
\end{array}\right) \in U .
$$

Then $\mathfrak{s}\left(\omega^{-1} h\right)=\mathfrak{s}(c) \mathfrak{s}(u)$. We find ${ }^{\mathfrak{s}(\omega)} \mathfrak{s}(c)$ using Remark 3.8. Write $\omega=t_{0} \mathfrak{w}_{\omega}$ with

$$
\begin{aligned}
& \mathfrak{w}_{\omega}=\left(\mathfrak{w}_{\alpha_{n-1}} \cdot \ldots \cdot \mathfrak{w}_{\alpha_{1}}\right) \cdot \ldots \cdot\left(\mathfrak{w}_{\alpha_{n-1}} \cdot \ldots \cdot \mathfrak{w}_{\alpha_{k}}\right)=\prod_{i=1}^{k}\left(\mathfrak{w}_{\alpha_{n-1}} \cdot \ldots \cdot \mathfrak{w}_{\alpha_{i}}\right) \in \mathfrak{W}, \\
& t_{0}=\operatorname{diag}\left((-1)^{k} I_{k},(-1)^{k-1},(-1)^{k-2}, \ldots, 1\right)
\end{aligned}
$$

If $w_{\omega}$ is the Weyl element corresponding to $\mathfrak{w}_{\omega}$ (or $\omega$ ),

$$
\left\{\alpha>0: w_{\omega} \alpha<0\right\}=\{(i, j): 1 \leq i \leq k, \quad i<j \leq n\} .
$$

Then

$$
\mathfrak{s}(\omega) \mathfrak{s}(c)=\left(-1, z^{-1}\right)_{2}^{k-1} \mathfrak{s}\left(t_{0}\right) \mathfrak{s}\left({ }^{\omega} c\right)=(z, z){ }_{2}^{k} \mathfrak{s}\left({ }^{\omega} c\right) .
$$

Consider ${ }^{\mathfrak{s}(\omega)} \mathfrak{s}(u)$. If $\omega^{\prime} \in G_{n}$ satisfies ${ }^{\omega^{\prime-1} \omega} u=u$, then ${ }^{\mathfrak{s}\left(\omega^{\prime-1} \omega\right)} \mathfrak{s}(u)=\mathfrak{s}(u)$ hence ${ }^{\mathfrak{s}(\omega)} \mathfrak{s}(u)=$ $\mathfrak{s}\left(\omega^{\prime}\right) \mathfrak{s}(u)$. One can take

$$
\omega^{\prime}=\operatorname{diag}\left(I_{k-1},(-1)^{k}, I_{k}\right) \mathfrak{w}_{\alpha_{k}} \cdot \ldots \cdot \mathfrak{w}_{\alpha_{n-1}} .
$$


The conjugation of $\mathfrak{s}(u)$ by $\mathfrak{s}\left(\mathfrak{w}_{\alpha_{k+1}} \cdot \ldots \cdot \mathfrak{w}_{\alpha_{n-1}}\right)$ commutes with $\mathfrak{s}\left(\right.$ i.e., ${ }^{\mathfrak{s}(\ldots)} \mathfrak{s}(u)=\mathfrak{s}(\cdots u)$, because it keeps $u$ in $N_{n}$. The last two conjugations are computed using (1.1) and (1.2),

$$
\mathfrak{s}\left(\left(\begin{array}{cc} 
& (-1)^{k-1} \\
1 &
\end{array}\right) \mathfrak{s}\left(\left(\begin{array}{cc}
1 & (-1)^{k-1} z^{-1} \\
1
\end{array}\right)\right) \mathfrak{s}\left(\left(\begin{array}{ll} 
& 1)^{k-1}
\end{array}\right)\right)=\mathfrak{s}\left(\left(\begin{array}{cc}
1 & \\
z^{-1} & 1
\end{array}\right)\right) .\right.
$$

Therefore ${ }^{\mathfrak{s}(\omega)} \mathfrak{s}(u)=\mathfrak{s}\left({ }^{\omega} u\right)$. Also by $(\underline{1.2}), \mathfrak{s}\left({ }^{\omega} c\right) \mathfrak{s}\left({ }^{\omega} u\right)=(z, z){ }_{2} \mathfrak{s}\left({ }^{\omega}(c u)\right)$. Altogether

$$
\mathfrak{h}(h)={ }^{\mathfrak{s}(\omega)}(\mathfrak{s}(c) \mathfrak{s}(u))=(z, z){ }_{2}^{k} \mathfrak{s}\left({ }^{\omega} c\right) \mathfrak{s}\left({ }^{\omega} u\right)=(z, z)_{2}^{k-1} \mathfrak{s}\left({ }^{\omega}(c u)\right)=(z, z)_{2}^{k-1} \mathfrak{s}(h) .
$$

Plugging this into (3.24), using (3.12) and Claim 3.18 yields

$$
\kappa(g)=\kappa\left(b_{0}\right) \mathfrak{s}\left(b_{*}\right)(z, z)_{2}^{k-1} \mathfrak{h}(h) \mathfrak{h}\left(h_{0}\right)=(z, z)_{2}^{k-1}\left(z, \operatorname{det} c_{h_{0}}\right)_{2} \mathfrak{s}\left(b_{0} b_{*}\right) \mathfrak{h}\left(h h_{0}\right),
$$

thereby giving (3.23).

We see that (3.18) becomes

$$
\operatorname{vol}(\mathcal{I} \mathfrak{w} \mathcal{I}) \sum_{l=0}^{\infty}\left(q^{2 s_{k}+1}\right)^{l} q^{-l}\left(\varpi^{-l}, \varpi^{-l}\right)_{2}^{k-1} \int_{\mathcal{O}^{*}} \gamma_{\psi^{\prime}}^{-1}\left(\varpi^{-l} x\right) \psi^{-1}\left(\varpi^{-l} x\right) d x .
$$

Note that $d x$ is the additive measure of $F$ (in particular $\int_{\mathcal{O}^{*}} d x=1-q^{-1}$ ).

Remark 3.24. This dx-integral is a particular instance of an integral studied by Szpruch Szp09, who used it to represent a local coefficient resembling Tate's $\gamma$-factor.

For $l>1$ the $d x$-integral vanishes, since

$$
\int_{\mathcal{O}^{*}} \gamma_{\psi^{\prime}}^{-1}\left(\varpi^{-l} x\right) \psi^{-1}\left(\varpi^{-l} x\right) d x=\gamma_{\psi^{\prime}}^{-1}\left(\varpi^{-l}\right) \sum_{\zeta \in(1+\varpi \mathcal{O}) \backslash \mathcal{O}^{*}}\left(\varpi^{-l}, \zeta\right) \int_{1+\varpi \mathcal{O}} \psi^{-1}\left(\varpi^{-l} \zeta x\right) d x=0,
$$

because the conductor of $\psi$ is $\mathcal{O}$. Consider $l=1$. Then (1.17) and the assumption $|\varrho|=1$ show $\gamma_{\psi^{\prime}}^{-1}\left(\varpi^{-1} x\right)=(-\varrho, \varpi)_{2} \gamma_{\psi}\left(\varpi^{-1} x\right)$, then $d x$-integral equals $(-\varrho, \varpi)_{2}$ multiplied by

$$
\int_{\mathcal{O}^{*}} \gamma_{\psi}\left(\varpi^{-1} x\right) \psi^{-1}\left(\varpi^{-1} x\right) d x
$$

This integral was computed in Szz09, Lemma 1.12] (this is $J_{1}\left(\mathbb{F}, \psi^{-1}, \chi^{0}\right)$ in his notation) and equals $q^{-1 / 2}$. Altogether we obtain

$$
\operatorname{vol}(\mathcal{I} \mathfrak{w} \mathcal{I})\left(1-q^{-1}+q^{2 s_{k}-1 / 2}(-\varrho, \varpi)_{2}(\varpi, \varpi)_{2}^{k-1}\right)
$$

Finally we prove (3.21). Let $\mathfrak{w}$ be the representative of $s_{\alpha_{i}} s_{\alpha_{n-i}}$. The argument is similar to the previous case, we point the relevant changes in the computation. Using (3.14) and the fact that $\mathfrak{w} \in H$,

$$
\mathcal{I} \mathfrak{w} \mathcal{I} \subset\left(B_{n} \cap K\right) \mathfrak{w} N_{\alpha_{i}}(\mathcal{O}) N_{\alpha_{n-i}}(\mathcal{O}) \mathfrak{w}^{-1}(H \cap K) .
$$

Then $g=b_{0} \mathfrak{w} v \mathfrak{w}^{-1} h_{0}$ with $v \in N_{\alpha_{i}}(\mathcal{O}) N_{\alpha_{n-i}}(\mathcal{O})\left(b_{0}, h_{0}\right.$ as above). Decompose $\mathfrak{w} v \mathfrak{w}^{-1}=b_{*} h$, now we compute using 2 blocks of $2 \times 2$ matrices, belonging to $G_{2} \times G_{2}$ embedded in $G_{n}$ such that its derived group is $\mathcal{R}_{\alpha_{i}} \times \mathcal{R}_{\alpha_{n-i}}$. For $v=\left(\left(\begin{array}{cc}1 & z_{1} \\ 1\end{array}\right),\left(\begin{array}{c}1 \\ z_{2} \\ 1\end{array}\right)\right)$ with $z_{1} z_{2} \neq 1$ and $\left(1-z_{1} z_{2}\right) \in F^{* 2} \mathcal{O}^{*}$,

$$
\begin{aligned}
& b_{*}=\left(\left(\begin{array}{cc}
\left(1-z_{1} z_{2}\right)^{-1} & z_{2}\left(1-z_{1} z_{2}\right)^{-1} \\
1
\end{array}\right),\left(\begin{array}{cc}
\left(1-z_{1} z_{2}\right)^{-1} & z_{1}\left(1-z_{1} z_{2}\right)^{-1} \\
1
\end{array}\right)\right), \\
& h=\left(\left(\begin{array}{cc}
1 & -z_{2} \\
-z_{1} & 1
\end{array}\right),\left(\begin{array}{cc}
1 & -z_{1} \\
-z_{2} & 1
\end{array}\right)\right) .
\end{aligned}
$$


When $\left(1-z_{1} z_{2}\right) \notin F^{* 2} \mathcal{O}^{*}$ we still have $\mathfrak{w} v \mathfrak{w}^{-1}=b_{*} h$ but $b_{*} \notin B_{n, *}$, and since

$$
g \in \operatorname{diag}\left(I_{i-1},\left(1-z_{1} z_{2}\right)^{-1},\left(1-z_{1} z_{2}\right), I_{n-i-1}\right) B_{n, *} H,
$$

by Claim 3.16 we obtain $g \notin B_{n, *} H$. Hence we can assume $1-z_{1} z_{2} \in F^{* 2} \mathcal{O}^{*}$. Then

$$
\begin{aligned}
& \psi^{-1}\left(u_{h_{g}}\right)=1, \\
& \gamma_{\psi^{\prime}}^{-1}\left(\operatorname{det} c_{h_{g}}\right)=\gamma_{\psi^{\prime}}^{-1}\left(1-z_{1} z_{2}\right)=1, \\
& \delta_{B_{n}}^{1 / 2}\left(b_{g}\right)=\left|1-z_{1} z_{2}\right|^{-1}, \\
& \chi\left(\mathfrak{s}\left(b_{g}\right)\right)=\left|1-z_{1} z_{2}\right|^{-s_{i}-s_{n-i}}=\left|1-z_{1} z_{2}\right|^{-s_{i}+s_{i+1}} .
\end{aligned}
$$

We may also take $z_{1} \neq 0$. Next we prove

$$
\mathfrak{s}\left(b_{g}\right) \mathfrak{h}\left(h_{g}\right)=\left(-z_{1}, 1-z_{1} z_{2}\right)_{2} \kappa(g),
$$

whence

$$
\operatorname{ch}_{s_{\alpha_{i}} s_{\alpha_{n-i}}, \chi^{-1}}\left(\mathfrak{s}\left(b_{g}\right) \mathfrak{h}\left(h_{g}\right)\right)=\left(-z_{1}, 1-z_{1} z_{2}\right)_{2} .
$$

As with the proof of (3.23), we begin with proving $\sigma\left(b_{*}, h\right)=1$. This is immediate if $z_{2}=0$. When $z_{2} \neq 0$, apply (1.2) separately to each $G_{2}$-block, then use (1.1). We obtain

$$
\left(-z_{1}, 1-z_{1} z_{2}\right)_{2}\left(-z_{2}, 1-z_{1} z_{2}\right)_{2}\left(\left(1-z_{1} z_{2}\right)^{-1},\left(1-z_{1} z_{2}\right)\right)_{2}=1 \text {. }
$$

Here we used the fact that $(x, 1-x)=1$ for any $x \neq 0$ and $1-z_{1} z_{2} \in F^{* 2} \mathcal{O}^{*}$. Next we show $\mathfrak{s}(h)=\left(z_{1}, 1-z_{1} z_{2}\right)_{2} \mathfrak{h}(h)$. Put $x=\left(\begin{array}{cc}1 & -z_{2} \\ -z_{1} & 1\end{array}\right)$. For any $g \in G_{2}$, let

$$
d_{1}(g)=\operatorname{diag}\left(I_{i-1}, g, I_{n-i-1}\right), \quad d_{2}(g)=\operatorname{diag}\left(I_{k+i-1}, g, I_{k-i-1}\right) .
$$

Then ${ }^{\omega^{-1}} h=d_{1}(x) d_{2}(x)$ and $\mathfrak{h}(h)={ }^{\mathfrak{s}(\omega)} \mathfrak{s}\left(d_{1}(x) d_{2}(x)\right)$. Now (1.1) implies

$$
\mathfrak{s}\left(d_{1}(x) d_{2}(x)\right)=\mathfrak{s}\left(d_{1}(x)\right) \mathfrak{s}\left(d_{2}(x)\right) \text {. }
$$

To compute ${ }^{\mathfrak{s}(\omega)} \mathfrak{s}\left(d_{j}(x)\right)$ we write $x$ using the Iwasawa decomposition in $G_{2}$,

$$
x=x_{1} x_{2} x_{3}=\left(\begin{array}{cc}
1-z_{1} z_{2} & \\
& 1
\end{array}\right)\left(\begin{array}{cc}
1 & -z_{2}\left(1-z_{1} z_{2}\right)^{-1} \\
1
\end{array}\right)\left(\begin{array}{cc}
1 & 0 \\
-z_{1} & 1
\end{array}\right) .
$$

Then by (1.2),

$$
\mathfrak{s}\left(d_{j}(x)\right)=\left(-z_{1}, 1-z_{1} z_{2}\right)_{2} \mathfrak{s}\left(d_{j}\left(x_{1}\right)\right) \mathfrak{s}\left(d_{j}\left(x_{2}\right)\right) \mathfrak{s}\left(d_{j}\left(x_{3}\right)\right), \quad j=1,2 .
$$

We compute the conjugation separately for each $x_{i}$ :

$\mathfrak{s}(\omega) \mathfrak{s}\left(d_{j}\left(x_{1}\right)\right)=\mathfrak{s}\left({ }^{\omega} d_{j}\left(x_{1}\right)\right)$ : If $\omega=t_{0} \mathfrak{w}_{\omega}$ as above, ${ }^{\mathfrak{s}(\mathfrak{w} \omega)} \mathfrak{s}\left(d_{j}\left(x_{1}\right)\right)=\mathfrak{s}\left({ }^{\mathfrak{w} \omega} d_{j}\left(x_{1}\right)\right)$ by Claim 3.7 and ${ }^{\mathfrak{s}\left(t_{0}\right)} \mathfrak{s}\left({ }^{\mathfrak{w} \omega} d_{j}\left(x_{1}\right)\right)=\mathfrak{s}\left({ }^{\mathfrak{w} \omega} d_{j}\left(x_{1}\right)\right)$ because $\left(-1,1-z_{1} z_{2}\right)_{2}=1\left(1-z_{1} z_{2} \in F^{* 2} \mathcal{O}^{*}\right)$. Hence $\mathfrak{s}(\omega) \mathfrak{s}\left(d_{j}\left(x_{1}\right)\right)=\mathfrak{s}\left({ }^{\omega} d_{j}\left(x_{1}\right)\right)$.

$\mathfrak{s}(\omega) \mathfrak{s}\left(d_{j}\left(x_{2}\right)\right)=\mathfrak{s}\left({ }^{\omega} d_{j}\left(x_{2}\right)\right)$ : For $j=1$ we argue as in the case of ${ }^{\mathfrak{s}(\omega)} \mathfrak{s}(u)$ above, this time with

$$
\omega^{\prime}=\operatorname{diag}\left(I_{n-i-1},-1, I_{i}\right) \mathfrak{w}_{\alpha_{n-i}} \cdot \ldots \cdot \mathfrak{w}_{\alpha_{i}} \mathfrak{w}_{\alpha_{n-i}} \cdot \ldots \cdot \mathfrak{w}_{\alpha_{i+1}},
$$

and obtain ${ }^{\mathfrak{s}(\omega)} \mathfrak{s}\left(d_{1}\left(x_{2}\right)\right)=\mathfrak{s}\left({ }^{\omega} d_{1}\left(x_{2}\right)\right)$. For $j=2$ this holds simply because ${ }^{\omega} d_{2}\left(x_{2}\right) \in N_{n}$.

$\mathfrak{s}(\omega) \mathfrak{s}\left(d_{j}\left(x_{3}\right)\right)=\mathfrak{s}\left({ }^{\omega} d_{j}\left(x_{3}\right)\right)$ : A direct computation using (1.2) shows

$$
\mathfrak{s}\left(x_{3}\right)=\mathfrak{s}\left(\begin{array}{cc} 
& -1 \\
1 &
\end{array}\right) \mathfrak{s}\left(\begin{array}{cc}
1 & z_{1} \\
& 1
\end{array}\right) \mathfrak{s}\left(\begin{array}{cc} 
& 1 \\
-1 &
\end{array}\right)=\kappa\left(x_{3}\right)
$$

$\left(z_{1} \in \mathcal{O}\right.$ and $\mathfrak{s}$ and $\kappa$ agree on $\left.N_{n} \cap K\right)$. Hence ${ }^{\mathfrak{s}(\omega)} \mathfrak{s}\left(d_{j}\left(x_{3}\right)\right)=\mathfrak{s}\left({ }^{\omega} d_{j}\left(x_{3}\right)\right)$. 
Summarizing,

$$
\mathfrak{s}^{\omega} \mathfrak{s} \mathfrak{s}\left(d_{j}(x)\right)=\left(-z_{1}, 1-z_{1} z_{2}\right)_{2} \mathfrak{s}\left({ }^{\omega} d_{j}\left(x_{1}\right)\right) \mathfrak{s}\left({ }^{\omega} d_{j}\left(x_{2}\right)\right) \mathfrak{s}\left({ }^{\omega} d_{j}\left(x_{3}\right)\right) .
$$

Similarly we see that

$$
\begin{aligned}
& \mathfrak{s}\left({ }^{\omega} d_{1}(x)\right)=\mathfrak{s}\left({ }^{\omega} d_{1}\left(x_{1}\right)\right) \mathfrak{s}\left({ }^{\omega} d_{1}\left(x_{2}\right)\right) \mathfrak{s}\left({ }^{\omega} d_{1}\left(x_{3}\right)\right), \\
& \mathfrak{s}\left({ }^{\omega} d_{2}(x)\right)=\left(-z_{1}, 1-z_{1} z_{2}\right){ }_{2} \mathfrak{s}\left({ }^{\omega} d\left(x_{1}\right)\right) \mathfrak{s}\left({ }^{\omega} d\left(x_{2}\right)\right) \mathfrak{s}\left({ }^{\omega} d\left(x_{3}\right)\right), \\
& \mathfrak{s}\left({ }^{\omega}\left(d_{1}(x) d_{2}(x)\right)\right)=\mathfrak{s}\left({ }^{\omega} d_{1}(x)\right) \mathfrak{s}\left({ }^{\omega} d_{2}(x)\right) .
\end{aligned}
$$

Thus (3.25)-(3.30) yield

$$
\begin{aligned}
\mathfrak{h}(h) & ={ }^{\mathfrak{s}(\omega)} \mathfrak{s}\left(d_{1}(x) d_{2}(x)\right) \\
& ={ }^{\mathfrak{s}(\omega)} \mathfrak{s}\left(d_{1}(x)\right){ }^{\mathfrak{s}(\omega)} \mathfrak{s}\left(d_{2}(x)\right) \\
& =\mathfrak{s}\left({ }^{\omega} d_{1}\left(x_{1}\right)\right) \mathfrak{s}\left({ }^{\omega} d_{1}\left(x_{2}\right)\right) \mathfrak{s}\left({ }^{\omega} d_{1}\left(x_{3}\right)\right) \mathfrak{s}\left({ }^{\omega} d_{2}\left(x_{1}\right)\right) \mathfrak{s}\left({ }^{\omega} d_{2}\left(x_{2}\right)\right) \mathfrak{s}\left({ }^{\omega} d_{2}\left(x_{3}\right)\right) \\
& =\left(-z_{1}, 1-z_{1} z_{2}\right)_{2} \mathfrak{s}\left({ }^{\omega} d_{1}(x)\right) \mathfrak{s}\left({ }^{\omega} d_{2}(x)\right) \\
& =\left(-z_{1}, 1-z_{1} z_{2}\right)_{2} \mathfrak{s}\left({ }^{\omega}\left(d_{1}(x) d_{2}(x)\right)\right) \\
& =\left(-z_{1}, 1-z_{1} z_{2}\right)_{2} \mathfrak{s}(h) .
\end{aligned}
$$

Altogether (3.18) becomes

$$
\operatorname{vol}(\mathcal{I} \mathfrak{w} \mathcal{I}) \int_{\mathcal{O}} \int_{\mathcal{O}}\left(-z_{1}, 1-z_{1} z_{2}\right)_{2}\left|1-z_{1} z_{2}\right|^{-s_{i}+s_{i+1}-1} \operatorname{ch}_{F^{* 2} \mathcal{O}^{*}}\left(1-z_{1} z_{2}\right) d z_{1} d z_{2}
$$

Here $\mathrm{ch}_{F^{* 2} \mathcal{O}^{*}}$ is the characteristic function of $F^{* 2} \mathcal{O}^{*}$. As in [Sak06] (proof of Proposition 8.2) we split the integral into the following sum,

$$
\int_{\mathcal{O}} \int_{\varpi \mathcal{O}} d z_{1} d z_{2}+\int_{\varpi \mathcal{O}} \int_{\mathcal{O}^{*}} d z_{1} d z_{2}+\int_{\mathcal{O}^{*}} \int_{\mathcal{O}^{*}}\left|1-z_{1} z_{2}\right|^{-s_{i}+s_{i+1}-1} \operatorname{ch}_{F^{* 2} \mathcal{O}^{*}}\left(1-z_{1} z_{2}\right) d z_{1} d z_{2} .
$$

We used the fact that when either $z_{1}$ or $z_{2}$ belongs to $\varpi \mathcal{O}$ (and both $z_{1}, z_{2} \in \mathcal{O}$ ), $\left|1-z_{1} z_{2}\right|=1$ and $1-z_{1} z_{2} \in F^{* 2}$, in particular $\left(-z_{1}, 1-z_{1} z_{2}\right)_{2}=1$. For the rightmost integral note that when $z_{1} \in \mathcal{O}^{*}, \operatorname{ch}_{F^{* 2} \mathcal{O}^{*}}\left(1-z_{1} z_{2}\right)=1$ implies $\left(-z_{1}, 1-z_{1} z_{2}\right)_{2}=1$. The first summand contributes $q^{-1}$, the second $q^{-1}\left(1-q^{-1}\right)$. We proceed with the integral on the right. After a change of variables it becomes

$$
\left(1-q^{-1}\right) \int_{\mathcal{O}^{*}}|1-z|^{-s_{i}+s_{i+1}-1} \operatorname{ch}_{F^{* 2} \mathcal{O}^{*}}(1-z) d z .
$$

Put $|1-z|=q^{-l}$ for some $l \geq 0$. Then $\operatorname{ch}_{F^{* 2} \mathcal{O}^{*}}(1-z) \neq 0$ if and only if $l$ is even. Hence we get

$$
\left(1-q^{-1}\right)\left(1-2 q^{-1}+\left(1-q^{-1}\right) \frac{q^{-2\left(s_{i+1}-s_{i}\right)}}{1-q^{-2\left(s_{i+1}-s_{i}\right)}}\right) .
$$

Summing up we obtain

$$
\operatorname{vol}(\mathcal{I} \mathfrak{w} \mathcal{I})\left(1-q^{-1}+q^{-2}+\left(1-q^{-1}\right)^{2} \frac{q^{-2\left(s_{i+1}-s_{i}\right)}}{1-q^{-2\left(s_{i+1}-s_{i}\right)}}\right) .
$$

The proof is complete. 
Recall that we assume $\chi$ satisfies (3.17). We say that $f_{\chi^{-1}}$ is a polynomial (resp. rational) section if its restriction to $\coprod_{t \in T_{n, *} \backslash T_{n}} \mathfrak{s}(t) \kappa(K)$ depends polynomially (resp. rationally) on $\chi^{-1}$, or more precisely on $q^{ \pm 2 s_{1}}, \ldots, q^{ \pm 2 s_{k}}$. The definition is independent of the actual choice of representatives of $T_{n, *} \backslash T_{n}$.

Proposition 3.25. The functional $\Lambda_{\chi}$ has a meromorphic continuation to all $\chi$ satisfying (3.17), in the sense that if $f_{\chi^{-1}}$ is a rational section, $\Lambda_{\chi}\left(f_{\chi^{-1}}\right)$ is a rational function, i.e., belongs to $\mathbb{C}\left(q^{-2 s_{1}}, \ldots, q^{-2 s_{k}}\right)$.

Proof. The argument appeared in [Sak06, § 7]. According to Theorem 3.10 and Claim 3.15, there exists at most one solution (a functional) to the system of equations, defined by (3.13) with the additional condition that $\varphi_{e, \chi^{-1}}$ is mapped to $\operatorname{vol}(\mathcal{I})$. Furthermore, according to Claim 3.21 and (3.19), in an open subset of $\mathbb{C}^{k}$, there exists a nontrivial solution. Hence by Bernstein's continuation principle (in [Ban98] $) \Lambda_{\chi}\left(f_{\chi^{-1}}\right)$ extends to a rational function.

Corollary 3.26. The results of Lemma 3.22 hold for all $\chi$ satisfying (3.17), by means of meromorphic continuation.

Below we state the explicit formula for the unramified function $g \mapsto \Lambda_{\chi}\left(g \varphi_{K, \chi^{-1}}\right)$. The formula is for $g=t_{\lambda}$ with $\lambda \in \mathbb{Z}_{\geq}^{k}$. The following simple claim shows that these elements already determine the values of the function.

Claim 3.27. Let $l$ be a metaplectic $\left(\psi^{\prime}, \psi\right)$-Shalika functional (resp. ( $\left.H, \psi^{\prime}, \psi\right)$-functional) on a genuine unramified $\pi \in \operatorname{Alg} \widetilde{G}_{n}$. Let $\xi$ be an unramified vector in the space of $\pi$ and consider the function $l_{\xi}(g)=l(\pi(g) \xi)$.

(1) $l_{\xi}\left(\kappa\left(k_{0}\right) g \kappa(k)\right)=l_{\xi}(g)$ for any $k_{0} \in\left(G_{k}^{\triangle} U\right) \cap K$ (resp. $\left.k_{0} \in H \cap K\right), g \in \widetilde{G}_{n}$ and $k \in K$.

(2) $l_{\xi}$ is uniquely determined by its values on $t_{\lambda}$ (resp. $t_{-\lambda}$ ), where $\lambda \in \mathbb{Z}_{\geq}^{k}$.

(3) $l_{\xi}\left(t_{\lambda}\right)=0$ (resp. $t_{-\lambda}$ ) for $\lambda \in \mathbb{Z}_{\geq}^{k}-\mathbb{Z}_{+}^{k}$.

Proof. The first assertion follows from Claim 3.18 and (3.13). The second and third follow by a straightforward adaptation of the proofs of these results in the non-metaplectic setting ([JR96, $§ 6.2])$. For example, we explain the proof of (3) for the $\left(H, \psi^{\prime}, \psi\right)$-functional. Consider $\lambda \in \mathbb{Z}_{\geq}^{k}$. Let $u \in \mathcal{O}_{k \times k}$ and $h={ }^{\omega}\left(\begin{array}{cc}I_{k} & u \\ & I_{k}\end{array}\right) \in H \cap K$. Then

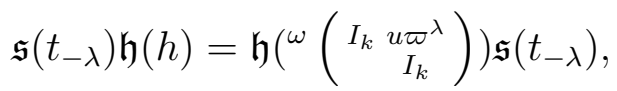

whence by Claim 3.18 ,

$$
l_{\xi}\left(\mathfrak{s}\left(t_{-\lambda}\right)\right)=l_{\xi}\left(\mathfrak{s}\left(t_{-\lambda}\right) \mathfrak{h}(h)\right)=\psi\left(\left(\begin{array}{cc}
I_{k} & u \varpi^{\lambda} \\
& I_{k}
\end{array}\right)\right) l_{\xi}\left(\mathfrak{s}\left(t_{-\lambda}\right)\right) .
$$

Thus $l_{\xi}\left(\mathfrak{s}\left(t_{-\lambda}\right)\right)=0$, unless $\lambda \in \mathbb{Z}_{+}^{k}$.

Let $\mathrm{Sp}_{k}$ be the symplectic group on $n$ variables, embedded in $G_{n}$ as the subgroup

$$
\left\{g \in G_{n}:{ }^{t} g\left({ }_{-J_{k}}^{J_{k}}\right) g=\left({ }_{-J_{k}}^{J_{k}}\right)\right\}
$$

where ${ }^{t} g$ denotes the transpose of $g$. We fix the Borel subgroup $\operatorname{Sp}_{k} \cap B_{n}$ and let $\Sigma_{\mathrm{Sp}_{\mathrm{k}}}$ (resp. $\Sigma_{\mathrm{Sp}_{k}}^{+}$) be the corresponding root system (resp. positive roots) of $\mathrm{Sp}_{k}$. The map $\Sigma_{G_{n}} \rightarrow \Sigma_{\mathrm{Sp}_{k}}$ is two-to-one onto the short roots, and injective onto the long roots. Recall that we assumed $q^{2 s_{i}}=q^{-2 s_{n-i+1}}$ for all $1 \leq i \leq k$ (in (3.17) $)$. Equivalently, $\chi$ is trivial on $\mathfrak{s}\left(H \cap B_{n, *}\right)$. Hence if $\beta, \beta^{\prime} \in \Sigma_{G_{n}}$ are the two roots corresponding to a short root $\alpha \in \Sigma_{\operatorname{Sp}_{k}}, c_{\beta}(\chi)=c_{\beta^{\prime}}(\chi)$. 
Thus we may extend the notation $c_{\alpha}(\chi)$ to short roots $\alpha$, meaning $c_{\beta}(\chi)$. The same applies to $\chi^{-1}$. Similarly, we define $c_{\alpha}(\chi)$ for long roots (there is no ambiguity here, for any $\chi$ ).

Denote the Weyl group of $\mathrm{Sp}_{k}$ by $W_{\mathrm{Sp}_{k}}$, it is the group generated by $s_{k}$ and $s_{i} s_{n-i}, 1 \leq$ $i<k$. For $w \in W_{\mathrm{Sp}_{k}}$, let $\ell_{\mathrm{Sp}_{k}}(w)$ be the minimal number of simple reflections in $W_{\mathrm{Sp}_{k}}$ whose product is $w$, i.e., the usual length with respect to $W_{\mathrm{Sp}_{k}}$. let

Recall that $\psi^{\prime}=\psi_{\varrho}$ (see Lemma [3.22), put $\epsilon_{\varrho, k}=(-\varrho, \varpi)_{2}(\varpi, \varpi)_{2}^{k-1}$. For any $\alpha \in \Sigma_{\mathrm{Sp}_{k}}^{+}$

$$
y_{\alpha}\left(\chi^{-1}\right)= \begin{cases}c_{\alpha}\left(\chi^{-1}\right) c_{\alpha}(\chi) & \alpha \text { is short, } \\ \frac{\left(1+q^{-1 / 2} \epsilon_{\varrho, k} \chi^{-1 / 2}\left(a_{\alpha}\right)\right)\left(1-q^{-1 / 2} \epsilon_{\varrho, k} \chi^{1 / 2}\left(a_{\alpha}\right)\right)}{1-\chi\left(a_{\alpha}\right)} & \alpha \text { is long. }\end{cases}
$$

As in Lemma 3.22, if $\alpha$ is the long root $(i, n-i+1), \chi^{-1 / 2}\left(a_{\alpha}\right)=q^{-2 s_{i}}$.

Theorem 3.28. Let $\lambda \in \mathbb{Z}_{\geq}^{k}$. The function $\Lambda_{\chi}\left(\mathfrak{s}\left(t_{-\lambda}\right) \varphi_{K, \chi^{-1}}\right)$ vanishes unless $\lambda \in 2 \mathbb{Z}_{+}^{k}$. In this case,

$$
\Lambda_{\chi}\left(\mathfrak{s}\left(t_{-\lambda}\right) \varphi_{K, \chi^{-1}}\right)=Q^{-1} \sum_{w \in W_{\mathrm{Sp}_{k}}} \prod_{\{\alpha>0: w \alpha>0\}} c_{\alpha}\left(\chi^{-1}\right) \prod_{\left\{\alpha \in \Sigma_{\mathrm{Sp}_{k}}^{+}: w \alpha<0\right\}} y_{\alpha}\left(\chi^{-1}\right) \delta_{B_{n}}^{1 / 2}{ }^{w} \chi\left(\mathfrak{s}\left(t_{\lambda}\right)\right) .
$$

More compactly, put

$$
\beta\left(\chi^{-1}\right)=\prod_{\alpha \in \Sigma_{\mathrm{S}_{k}}^{+}} \chi^{1 / 2}\left(a_{\alpha}\right) \prod_{\text {short }}^{+} \prod_{\alpha \in \Sigma_{\mathrm{S}_{k}}^{+}}\left(1-q^{-1} \chi^{-1}\left(a_{\alpha}\right)\right) \prod_{\text {long } \alpha \in \Sigma_{\mathrm{S}_{k}}^{+}}\left(1-q^{-1 / 2} \epsilon_{\varrho, k} \chi^{-1 / 2}\left(a_{\alpha}\right)\right) .
$$

Then

$$
\Lambda_{\chi}\left(\mathfrak{s}\left(t_{-\lambda}\right) \varphi_{K, \chi^{-1}}\right)=Q^{-1} c_{w_{0}}\left(\chi^{-1}\right) \beta\left(\chi^{-1}\right)^{-1} \sum_{w \in W_{\mathrm{Sp}_{k}}}(-1)^{\ell_{\mathrm{Sp}_{k}}(w)} \beta\left({ }^{w} \chi^{-1}\right) \delta_{B_{n}}^{1 / 2}{ }^{w} \chi\left(\mathfrak{s}\left(t_{\lambda}\right)\right) .
$$

Remark 3.29. The product over $\chi^{1 / 2}\left(a_{\alpha}\right)$ in this formula is well defined because

$$
\frac{\prod_{\alpha \in \Sigma_{\mathrm{Sp}_{k}}^{+}}{ }^{w} \chi^{1 / 2}\left(a_{\alpha}\right)}{\prod_{\alpha \in \Sigma_{\mathrm{Sp}_{k}}^{+}} \chi^{1 / 2}\left(a_{\alpha}\right)}=\prod_{\left\{\alpha \in \Sigma_{\mathrm{Sp}_{k}}^{+}: w \alpha<0\right\}} \chi^{-1}\left(a_{\alpha}\right)
$$

Remark 3.30. To obtain the formula for the (non-metaplectic) Shalika model, simply remove the product over long roots in the definition of $\beta\left(\chi^{-1}\right)$. Up to a factor independent of $\lambda$ this was the formula obtained by Sakellaridis [Sak06] (see also [Sak13, § 5.5.2]).

Proof. We use the notation of $\S 2.3$. The space of $\left(H, \psi^{\prime}, \psi\right)$-functionals on $\mathrm{I}\left(\chi^{-1}\right)$ is at most one-dimensional. Therefore (2.15) becomes

$$
T_{w^{-1},{ }^{w} \chi^{-1}} \Lambda_{\chi}=A(w, \chi) \Lambda_{w},
$$

where $A(w, \chi)$ is a scalar (a rational function of $\chi$ ). By virtue of Corollary 2.17 ,

$$
\Lambda_{\chi}\left(\mathfrak{s}\left(t_{-\lambda}\right) \varphi_{K, \chi^{-1}}\right)=\frac{1}{Q \operatorname{vol}(\mathcal{I})} \sum_{w \in W} \frac{c_{w_{0}}\left({ }^{w_{0} w} \chi\right)}{c_{w^{-1}}\left({ }^{w} \chi^{-1}\right)} A(w, \chi)\left(\Lambda_{w_{\chi}}\left(\mathfrak{s}\left(t_{-\lambda}\right) \varphi_{e, \chi^{-1}}\right) .\right.
$$

According to Lemma 3.22 ,

$$
\Lambda_{w \chi}\left(\mathfrak{s}\left(t_{-\lambda}\right) \varphi_{e, \chi^{-1}}\right)= \begin{cases}\operatorname{vol}(\mathcal{I}) \delta_{B_{n}}^{1 / 2}{ }^{w} \chi\left(\mathfrak{s}\left(t_{\lambda}\right)\right) & \lambda \in 2 \mathbb{Z}_{+}^{k}, \\ 0 & \lambda \in \mathbb{Z}_{+}^{k}-2 \mathbb{Z}_{+}^{k} .\end{cases}
$$


More precisely $\Lambda_{w} \chi$ is a functional on $\mathrm{I}\left({ }^{w} \chi^{-1}\right)$, and the domain of absolute convergence changes after the conjugation, so actually we apply Corollary 3.26 and deduce this equality in the sense of meromorphic continuation.

Therefore $\Lambda_{\chi}\left(\mathfrak{s}\left(t_{-\lambda}\right) \varphi_{K, \chi^{-1}}\right)$ vanishes, unless $\lambda \in 2 \mathbb{Z}_{+}^{k}$. Henceforth assume this is the case. Equality (3.32) also implies $\Lambda_{w}\left(\varphi_{e, \chi^{-1}}\right) \neq 0$. Therefore $A(w, \chi)$ is given by the quotient

$$
A(w, \chi)=\frac{T_{w^{-1},{ }^{w} \chi^{-1}} \Lambda_{\chi}\left(\varphi_{\left.e,{ }^{w} \chi^{-1}\right)}\right.}{\Lambda{ }{ }_{\chi}\left(\varphi_{e,{ }^{w} \chi^{-1}}\right)}=\frac{\Lambda_{\chi}\left(T_{w^{-1},{ }^{w} \chi^{-1}} \varphi_{e,{ }^{w} \chi^{-1}}\right)}{\operatorname{vol}(\mathcal{I})} .
$$

We claim that only elements of $W_{\mathrm{Sp}_{k}}$ contribute to the sum.

Claim 3.31. If $w \in W-W_{\mathrm{Sp}_{k}}, A(w, \chi)=0$.

Using the fact that $c_{w_{0}}\left({ }^{w_{0} w} \chi\right)=c_{w_{0}}\left({ }^{w} \chi^{-1}\right)$, we obtain (this is [Sak06, (48)])

$$
\Lambda_{\chi}\left(\mathfrak{s}\left(t_{-\lambda}\right) \varphi_{K, \chi^{-1}}\right)=Q^{-1} \sum_{w \in W_{\mathrm{S}_{k}}} \prod_{\{\alpha>0: w \alpha>0\}} c_{\alpha}\left(\chi^{-1}\right) A(w, \chi) \delta_{B_{n}}^{1 / 2}{ }^{w} \chi\left(\mathfrak{s}\left(t_{\lambda}\right)\right) .
$$

It remains to compute $A(w, \chi)$ for $w \in W_{\mathrm{Sp}_{k}}$. Let $\ell_{\mathrm{Sp}_{k}}(w)=l$ and write $w=s_{1}^{\prime} \cdot \ldots \cdot s_{l}^{\prime}$, where $s_{i}^{\prime}$ is either $s_{\alpha_{k}}$ or $s_{\alpha_{i}} s_{\alpha_{n-i}}$ with $1 \leq i<k$. Then

$$
A(w, \chi)=A\left(s_{1}^{\prime},{ }^{\prime} \cdot \ldots \cdot s_{l}^{\prime} \chi\right) \ldots A\left(s_{l-1}^{\prime},{ }^{\prime} \chi\right) A\left(s_{l}^{\prime}, \chi\right) .
$$

We will compute $A\left(s_{i}^{\prime}, \chi\right)$ succinctly using Claim 2.9 and Corollary 3.26.

Claim 3.32. We have $A\left(s_{\alpha_{k}}, \chi\right)=y_{\alpha_{k}}\left(\chi^{-1}\right)$ and $A\left(s_{\alpha_{i}} s_{\alpha_{n-i}}, \chi\right)=y_{\alpha_{i}}\left(\chi^{-1}\right)$.

We conclude that for any $w \in W_{\mathrm{Sp}_{k}}$,

$$
A(w, \chi)=\prod_{\left\{\alpha \in \Sigma_{\mathrm{Sp}_{k}}^{+}: w \alpha<0\right\}} y_{\alpha}\left(\chi^{-1}\right) .
$$

Plugging this into the last expression for $\Lambda_{\chi}\left(\mathfrak{s}\left(t_{-\lambda}\right) \varphi_{K, \chi^{-1}}\right)$ yields the theorem. To obtain the compact form with $\beta\left(\chi^{-1}\right)$ one can apply manipulations similar to [Sak06, (75)-(78)].

Proof of Claim 3.31. According to (3.33) it is enough to show that $T_{w^{-1},{ }^{w} \chi^{-1}} \Lambda_{\chi}$ vanishes on $\varphi_{e,{ }^{w} \chi^{-1}}$. By (3.15) the support of $\varphi_{e,{ }^{w} \chi^{-1}}$ is contained in $\widetilde{B}_{n, *} H$. Let $\Lambda$ denote the restriction of $T_{w^{-1}{ }^{w}{ }^{w} \chi^{-1}} \Lambda_{\chi}$ to the subspace of functions in $\mathrm{I}\left({ }^{w} \chi^{-1}\right)$, whose support is contained in $\widetilde{B}_{n, *} H$. We prove $\Lambda=0$. The argument was adapted from [Sak06, Proposition 5.2].

Consider the space $\mathcal{S}^{\text {gen }}\left(p^{-1}\left(B_{n, *} H\right), \delta_{B_{n}}^{1 / 2}{ }^{w} \chi^{-1}\right)$ of complex-valued locally constant genuine functions $f$ on $p^{-1}\left(B_{n, *} H\right)$ such that

$$
f\left(\mathfrak{s}\left(b_{*}\right) h\right)=\delta_{B_{n}}^{1 / 2}{ }^{w} \chi^{-1}\left(\mathfrak{s}\left(b_{*}\right)\right) f(h), \quad \forall b_{*} \in B_{n, *}, h \in H .
$$

Since $B_{n, *} H$ is open in $G_{n}$ (Claim 3.17),$\Lambda$ is the restriction of $T_{w^{-1},{ }^{w} \chi^{-1}} \Lambda_{\chi}$ to this subspace.

Also consider $\mathcal{S}^{\text {gen }}\left(\mathfrak{s}\left(H \cap B_{n, *}\right) \backslash \widetilde{H},{ }^{w} \chi^{-1}\right)$, where the functions satisfy (3.34) on $b_{*} \in B_{n, *} \cap$ $H$, and note that $\delta_{B_{n}}$ is trivial there.

These spaces are isomorphic: in one direction use restriction to $\widetilde{H}$, conversely one can extend a function $f$ in $\mathcal{S}^{\text {gen }}\left(\mathfrak{s}\left(H \cap B_{n, *}\right) \backslash \widetilde{H},{ }^{w} \chi^{-1}\right)$ to the former space using

$$
\mathfrak{s}\left(b_{*}\right) \mathfrak{h}(h) \mapsto \delta_{B_{n}}^{1 / 2}{ }^{w} \chi^{-1}\left(\mathfrak{s}\left(b_{*}\right)\right) f(\mathfrak{h}(h))
$$

(to see this is well defined use (3.12)). 
Thus we may regard $\Lambda$ as a functional on $\mathcal{S}^{\text {gen }}\left(\mathfrak{s}\left(H \cap B_{n, *}\right) \backslash \widetilde{H},{ }^{w} \chi^{-1}\right)$. Then it lifts to a functional on $\mathcal{S}^{\text {gen }}(\widetilde{H})$, by virtue of the projection

$$
f^{B_{n, *} \cap H,{ }^{w} \chi^{-1}}(h)=\int_{B_{n, *} \cap H}{ }^{w} \chi\left(\mathfrak{s}\left(b_{*}\right)\right) f\left(\mathfrak{s}\left(b_{*}\right) h\right) d b_{*}, \quad h \in \widetilde{H}, f \in \mathcal{S}^{\text {gen }}(\widetilde{H}) .
$$

Furthermore, since $\Lambda$ is an $\left(H, \psi^{\prime}, \psi\right)$-functional, it is a scalar multiple of the functional $l_{H}$ (defined after Claim 3.20). Indeed, this follows from the Frobenius reciprocity: if we let $\iota$ be the identity character of $\mu_{2}$ and regard $\gamma_{\psi^{\prime}} \otimes \psi \otimes \iota$ as the genuine character of $\widetilde{H}$ taking $\mathfrak{h}(h)$ to $\gamma_{\psi^{\prime}}\left(c_{h}\right) \psi\left(u_{h}\right)$, both $\Lambda$ and $l_{H}$ belong to

$$
\operatorname{Hom}_{\widetilde{H}}\left(\mathcal{S}^{\text {gen }}(\widetilde{H}), \gamma_{\psi^{\prime}} \otimes \psi \otimes \iota\right)=\operatorname{Hom}_{\widetilde{H}}\left(\operatorname{ind}_{\mu_{2}}^{\widetilde{H}}(\iota), \gamma_{\psi^{\prime}} \otimes \psi \otimes \iota\right)=\operatorname{Hom}_{\mu_{2}}(\iota, \iota) .
$$

Finally for $b_{*} \in B_{n, *} \cap H$ and $f \in \mathcal{S}^{\text {gen }}(\widetilde{H})$, let $L\left(b_{*}\right) f(h)=f\left(\mathfrak{s}\left(b_{*}\right) h\right)$. Since $H$ is unimodular we see that $l_{H}\left(L\left(b_{*}\right) f\right)=l_{H}(f)$, so assuming $\Lambda \neq 0$, the same holds for $\Lambda$. But this contradicts the fact that $\Lambda$ factors through $\mathcal{S}^{\text {gen }}\left(\mathfrak{s}\left(H \cap B_{n, *}\right) \backslash \widetilde{H},{ }^{w} \chi^{-1}\right)$, because for $w \in W-W_{\mathrm{Sp}_{k}},{ }^{w} \chi^{-1}$ is not trivial on $\mathfrak{s}\left(H \cap B_{n, *}\right)$.

Proof of Claim 3.32. First note that by Claim 2.9 , for any $\alpha \in \Delta_{G_{n}}$,

$$
T_{s_{\alpha}} \varphi_{e,{ }^{s \alpha} \chi^{-1}}=\left(c_{\alpha}\left({ }^{s_{\alpha}} \chi^{-1}\right)-1\right) \varphi_{e, \chi^{-1}}+q^{-1} \varphi_{s_{\alpha}, \chi^{-1}} .
$$

Consider $A\left(s_{\alpha_{k}}, \chi\right)$. Assume that $\mathfrak{w}$ represents $s_{\alpha_{k}}$. We apply $\Lambda_{\chi}$ to (3.35) with $\alpha=\alpha_{k}$. By Corollary 3.26,

$$
\begin{aligned}
\Lambda_{\chi}\left(T_{s_{\alpha_{k}}} \varphi_{\left.e,{ }^{s_{\alpha_{k}}} \chi^{-1}\right)=}\right. & \operatorname{vol}(\mathcal{I})\left(c_{\alpha_{k}}\left({ }^{s_{\alpha_{k}}} \chi^{-1}\right)-1\right) \\
& +q^{-1} \operatorname{vol}(\mathcal{I} \mathfrak{w} \mathcal{I})\left(1-q^{-1}+(-\varrho, \varpi)_{2}(\varpi, \varpi)_{2}^{k-1} q^{-1 / 2} \chi^{-1 / 2}\left(a_{\alpha_{k}}\right)\right) .
\end{aligned}
$$

Since $c_{\alpha_{k}}\left(s_{\alpha_{k}} \chi^{-1}\right)=c_{\alpha_{k}}(\chi)$ and $\operatorname{vol}(\mathcal{I} \mathfrak{w} \mathcal{I})=q \cdot \operatorname{vol}(\mathcal{I})$, we find that

$$
\begin{aligned}
A\left(s_{\alpha_{k}}, \chi\right) & =c_{\alpha_{k}}(\chi)-q^{-1}+(-\varrho, \varpi)_{2}(\varpi, \varpi)_{2}^{k-1} q^{-1 / 2} \chi^{-1 / 2}\left(a_{\alpha_{k}}\right) \\
& =-\chi^{-1}\left(a_{\alpha_{k}}\right) \frac{\left(1+q^{-1 / 2} \epsilon_{\varrho, k} \chi^{-1 / 2}\left(a_{\alpha_{k}}\right)\right)\left(1-q^{-1 / 2} \epsilon_{\varrho, k} \chi^{1 / 2}\left(a_{\alpha_{k}}\right)\right)}{1-\chi^{-1}\left(a_{\alpha_{k}}\right)}=y_{\alpha_{k}}\left(\chi^{-1}\right) .
\end{aligned}
$$

We turn to $A\left(s_{\alpha_{i}} s_{\alpha_{n-i}}, \chi\right)$. Applying Claim 2.9 twice,

$$
\begin{aligned}
T_{s_{\alpha_{i}} s_{\alpha_{n-i}}} \varphi_{e,{ }^{s}{ }_{\alpha_{i}} s_{\alpha_{n-i}} \chi^{-1}}= & \left(c_{\alpha_{n-i}}\left({ }^{s_{\alpha_{n-i}}} \chi^{-1}\right)-1\right)\left(c_{\alpha_{i}}\left({ }^{s_{\alpha_{i}} s_{\alpha_{n-i}}} \chi^{-1}\right)-1\right) \varphi_{e, \chi^{-1}} \\
& +q^{-1}\left(c_{\alpha_{i}}\left({ }^{s_{\alpha_{i}} s_{\alpha_{n-i}}} \chi^{-1}\right)-1\right) \varphi_{s_{\alpha_{n-i}}, \chi^{-1}} \\
& +q^{-1}\left(c_{\alpha_{n-i}}\left({ }^{s_{\alpha_{n-i}}} \chi^{-1}\right)-1\right) \varphi_{s_{\alpha_{i}}, \chi^{-1}} \\
& +q^{-2} \varphi_{s_{\alpha_{i}} s_{\alpha_{n-i}}, \chi^{-1}}
\end{aligned}
$$

As in [Sak06] the value of $\Lambda_{\chi}$ on the second and third summands is already determined: Claim 3.31 implies that $\Lambda_{\chi}$ vanishes on $T_{s_{\alpha_{i}}} \varphi_{e,{ }^{s \alpha_{i}} \chi^{-1}}$ hence when we apply $\Lambda_{\chi}$ to (3.35) with $\alpha=\alpha_{i}$

$$
\Lambda_{\chi}\left(\varphi_{s_{\alpha_{i}}, \chi^{-1}}\right)=q\left(1-c_{\alpha_{i}}\left({ }^{s_{\alpha_{i}}} \chi^{-1}\right)\right) \Lambda_{\chi}\left(\varphi_{e, \chi^{-1}}\right)=\operatorname{vol}(\mathcal{I}) q\left(1-c_{\alpha_{i}}\left({ }^{s_{\alpha_{i}}} \chi^{-1}\right)\right) .
$$

Similarly

$$
\Lambda_{\chi}\left(\varphi_{s_{\alpha_{n-i}}, \chi^{-1}}\right)=\operatorname{vol}(\mathcal{I}) q\left(1-c_{\alpha_{n-i}}\left({ }^{s_{\alpha_{n-i}}} \chi^{-1}\right)\right)
$$


Also by Corollary 3.26 ,

$$
\Lambda_{\chi}\left(\varphi_{s_{\alpha_{i}} s_{\alpha_{n-i}}, \chi^{-1}}\right)=\operatorname{vol}(\mathcal{I} \mathfrak{w} \mathcal{I})\left(1-q^{-1}+q^{-2}+\left(1-q^{-1}\right)^{2} \frac{\chi^{-1}\left(a_{\alpha_{i}}\right)}{1-\chi^{-1}\left(a_{\alpha_{i}}\right)}\right) .
$$

Note that now $\operatorname{vol}(\mathcal{I} \mathfrak{w} \mathcal{I})=q^{2} \operatorname{vol}(\mathcal{I})$. Plugging these identities into (3.36) and using the fact that $c_{\alpha_{n-i}}(\chi)=c_{\alpha_{i}}(\chi)$ (because $s_{n-i}=-s_{i+1}$ and $s_{n-i+1}=-s_{i}$ ) yields

$$
\begin{aligned}
& \Lambda_{\chi}\left(T_{s_{\alpha_{i}} s_{\alpha_{n-i}}} \varphi_{e,}{ }^{s \alpha_{i}{ }^{s} \alpha_{n-i}} \chi^{-1}\right) \\
& =\operatorname{vol}(\mathcal{I})\left(-\left(c_{\alpha_{i}}(\chi)-1\right)^{2}+1-q^{-1}+q^{-2}+\left(1-q^{-1}\right)^{2} \frac{\chi^{-1}\left(a_{\alpha_{i}}\right)}{1-\chi^{-1}\left(a_{\alpha_{i}}\right)}\right) .
\end{aligned}
$$

We see that

$$
A\left(s_{\alpha_{i}} s_{\alpha_{n-i}}, \chi\right)=-\chi^{-1}\left(a_{\alpha_{i}}\right) \frac{1-q^{-1} \chi\left(a_{\alpha_{i}}\right)}{1-q^{-1} \chi^{-1}\left(a_{\alpha_{i}}\right)} c_{\alpha_{i}}\left(\chi^{-1}\right)^{2}=c_{\alpha_{i}}(\chi) c_{\alpha_{i}}\left(\chi^{-1}\right)=y_{\alpha_{i}}\left(\chi^{-1}\right),
$$

completing the proof of the claim.

The following corollary strengthens Proposition 3.25.

Corollary 3.33. Let $\chi$ be a character satisfying (3.17). Then $\Lambda_{\chi}$ is a nonzero $\left(H, \psi^{\prime}, \psi\right)$ functional on $\mathrm{I}\left(\chi^{-1}\right)$. Moreover it depends polynomially on $\chi$, in the sense that for a polynomial section $f_{\chi^{-1}}, \Lambda_{\chi}\left(f_{\chi^{-1}}\right)$ belongs to $\mathbb{C}\left[q^{-2 s_{1}}, \ldots, q^{-2 s_{k}}\right]$.

Proof. According to Proposition 3.25, the functional $\Lambda_{\chi}$ is defined on $\mathrm{I}\left(\chi^{-1}\right)$ by means of meromorphic continuation. It is nonzero because it does not vanish on $\varphi_{e, \chi^{-1}}$ (Corollary [3.26). It remains to show it does not have a pole. Claim 3.27 and Theorem 3.28 show that there are no poles on the $\widetilde{G}_{n^{-}}$-space spanned by $\varphi_{K, \chi^{-1}}$. Claim 2.4 implies that for almost all $\chi$ (except for a discrete subset of $\left.\mathbb{C}^{k}\right)$, this space is all of $\mathrm{I}\left(\chi^{-1}\right)$. Since $\Lambda_{\chi}$ is determined by meromorphic continuation, the assertion holds.

Remark 3.34. The representation $\mathrm{I}\left(\chi^{-1}\right)$ might be reducible. In this case it may happen that

$$
\prod_{\{\alpha>0: w \alpha>0\}} c_{\alpha}\left(\chi^{-1}\right) \prod_{\left\{\alpha \in \Sigma_{\mathrm{Sp}_{k}}^{+}: w \alpha<0\right\}} y_{\alpha}\left(\chi^{-1}\right)=0, \quad \forall w \in W_{\mathrm{Sp}_{k}},
$$

and consequently $\Lambda_{\chi}$ will vanish on the $\widetilde{G}_{n}$-space spanned by $\varphi_{K, \chi^{-1}}$.

We finally relate between the $\left(H, \psi^{\prime}, \psi\right)$-functional and the metaplectic $\left(\psi^{\prime}, \psi\right)$-Shalika functional, on the unramified normalized element of $\mathrm{I}\left(\chi^{-1}\right)$.

Corollary 3.35. Assume $\chi$ satisfies (3.17) and let $l$ be the functional on $\mathrm{I}\left(\chi^{-1}\right)$ given by

$$
l\left(f_{\chi^{-1}}\right)=\Lambda_{\chi}\left(\mathfrak{s}(w) f_{\chi^{-1}}\right) .
$$

(1) $l$ is a nonzero metaplectic $\left(\psi^{\prime}, \psi\right)$-Shalika functional.

(2) $l$ depends polynomially on $\chi$.

(3) $l\left(\mathfrak{s}\left(t_{\lambda}\right) \varphi_{K, \chi^{-1}}\right)=\Lambda_{\chi}\left(\mathfrak{s}\left(t_{-\lambda}\right)\right)$ for all $\lambda \in \mathbb{Z}_{\geq}^{k}$. In particular $l\left(\mathfrak{s}\left(t_{\lambda}\right) \varphi_{K, \chi^{-1}}\right)$ vanishes on $\lambda \in \mathbb{Z}_{\geq}^{k}-2 \mathbb{Z}_{+}^{k}$. Consequently, Theorem 3.28 gives the Casselman-Shalika formula for the metaplectic Shalika model. 
Proof. We adapt the argument from [Sak06, (25)]. We have $h^{w} t_{\lambda}=t_{-\lambda}$ with

$$
h=\operatorname{diag}\left(\varpi^{-\lambda}, J_{k} \varpi^{-\lambda} J_{k}\right) \in H .
$$

Put $\mathfrak{h}(h)^{\mathfrak{s}(w)} \mathfrak{s}\left(t_{\lambda}\right)=\epsilon \mathfrak{s}\left(t_{-\lambda}\right)$ for some $\epsilon \in \mu_{2}$. Because $\varphi_{K, \chi^{-1}}$ is unramified,

$$
\left.l\left(\mathfrak{s}\left(t_{\lambda}\right) \varphi_{K, \chi^{-1}}\right)=\Lambda_{\chi}{ }^{\mathfrak{s}(w)} \mathfrak{s}\left(t_{\lambda}\right) \varphi_{K, \chi^{-1}}\right)=\epsilon \gamma_{\psi^{\prime}}\left(\operatorname{det} c_{h}\right)^{-1} \Lambda_{\chi}\left(\mathfrak{s}\left(t_{-\lambda}\right) \varphi_{K, \chi^{-1}}\right) .
$$

This vanishes unless $\lambda \in 2 \mathbb{Z}_{+}^{k}$, but then $\epsilon=1$ and $\gamma_{\psi^{\prime}}\left(\operatorname{det} \varpi^{\lambda}\right)=1$. The result follows.

3.4. Archimedean uniqueness results. In this section we establish uniqueness results for the metaplectic Shalika model of a principal series representation, analogous to those of Theorem 3.10, We assume $F=\mathbb{R}$, but the proof applies to $\mathbb{C}$ as well. In fact, we only need the real case, because we will apply this result to the exceptional representation, which is irreducible, and then over $\mathbb{C}$ uniqueness was proved in AGJ09.

Over $\mathbb{R}$ it is convenient to work with the Langlands decomposition $B_{n}=T_{n} N_{n}=\mathcal{M}_{n} A_{n} N_{n}$, where

$$
\begin{aligned}
& \mathcal{M}_{n}=\left\{\operatorname{diag}\left(t_{1}, \ldots, t_{n}\right): t_{i} \in \mu_{2}\right\} \cong \mu_{2}^{n}, \\
& A_{n}=\left\{\operatorname{diag}\left(t_{1}, \ldots, t_{n}\right): t_{i} \in \mathbb{R}_{>0}\right\} \cong \mathbb{R}_{>0}^{n} .
\end{aligned}
$$

Note that $A_{n}$ splits under the cover. The group $\widetilde{\mathcal{M}}_{n}$ is a non-trivial extension of $\mathcal{M}_{n}$ which we now describe in more detail. Let $C(n)$ denote the Clifford algebra in $n$ generators $e_{1}, \ldots, e_{n}$ with relations $e_{i}^{2}=1$ and $e_{i} e_{j}+e_{j} e_{i}=0$. Then

$$
\widetilde{\mathcal{M}}_{n} \cong\left\{ \pm e_{i_{1}} \cdots e_{i_{r}}: 1 \leq i_{1}<\ldots<i_{r} \leq n\right\} .
$$

The covering map $\widetilde{\mathcal{M}}_{n} \rightarrow \mathcal{M}_{n}$ is given by $\pm e_{i_{1}} \cdots e_{i_{r}} \mapsto \varepsilon_{I}$ with $I=\left\{i_{1}, \ldots, i_{r}\right\}$, where $\varepsilon_{I}$ is the diagonal matrix having -1 on the diagonal at positions $i_{1}, \ldots, i_{r}$, and 1 at the remaining ones. Further, the section $\mathfrak{s}$ is on $\mathcal{M}_{n}$ given by

$$
\mathfrak{s}\left(\varepsilon_{I}\right)=e_{i_{r}} \cdots e_{i_{1}}=(-1)^{\frac{r(r-1)}{2}} e_{i_{1}} \cdots e_{i_{r}} .
$$

Claim 3.36. The group $\widetilde{\mathcal{M}}_{n}$ has $2^{n}+1$ irreducible representations, $2^{n}$ non-genuine characters and one genuine representation of dimension $2^{k}$. The genuine representation $\sigma$ is the restriction of the pin-representation of $\operatorname{Pin}(n) \subset C(n)$ to $\widetilde{\mathcal{M}}_{n}$.

Proof. It is easy to see that $\widetilde{\mathcal{M}}_{n}$ has $2^{n}+1$ conjugacy classes and hence $2^{n}+1$ irreducible representations. Since $\mathcal{M}_{n} \cong \mu_{2}^{n}$ there are $2^{n}$ non-isomorphic characters of $\mathcal{M}_{n}$ which lift to non-isomorphic characters of $\widetilde{\mathcal{M}}_{n}$. Since the squares of the dimensions of all irreducible representations of $\widetilde{\mathcal{M}}_{n}$ sum up to the order of $\widetilde{\mathcal{M}}_{n}$ the last remaining irreducible representation must have dimension $2^{k}$. The restriction of the pin-representation to $\widetilde{\mathcal{M}}_{n}$ is easily seen to be genuine and has dimension $2^{k}$, hence has to be irreducible by a complete reducibility argument.

The genuine principal series of $\widetilde{G}_{n}$ is given by

$$
J(\nu)=\operatorname{Ind}{\widetilde{\mathcal{M}_{n}}}_{n} A_{n} N_{n}\left(\sigma \otimes e^{\nu+\rho} \otimes 1\right),
$$

where $\nu=\left(\nu_{1}, \ldots, \nu_{n}\right) \in \mathbb{C}^{n}$ corresponds to the character of the Lie algebra $\mathfrak{a}_{n}$ of $A_{n}$ given by $\nu\left(\operatorname{diag}\left(t_{1}, \ldots, t_{n}\right)\right)=\sum_{j=1}^{n} \nu_{j} t_{j}$, and $\rho=\left(\rho_{1}, \ldots, \rho_{n}\right)=\left(\frac{n-1}{2}, \frac{n-3}{2}, \ldots, \frac{1-n}{2}\right)$ denotes half the sum of all positive roots. 
Theorem 3.37. Assume that $\nu \in \mathbb{C}^{n}$ satisfies the condition

$$
\nu_{i}+\nu_{j} \neq 0, \quad \forall 1 \leq i \leq k, 1 \leq j \leq n \text { with } j \neq i, n-i+1 .
$$

Then the space of metaplectic Shalika functionals on $J(\nu)$ is at most one-dimensional. Furthermore, it is trivial unless $\nu_{i}+\nu_{n-i+1}=0$ for all $1 \leq i \leq k$.

Proof. By Archimedean Bruhat theory we have for any two closed subgroups $H_{1}, H_{2} \subset \widetilde{G}_{n}$ and any two (finite-dimensional) representations $\xi_{1}$ and $\xi_{2}$ of $H_{1}$ and $H_{2}$ that

$$
\begin{aligned}
\operatorname{dim} \operatorname{Hom}_{\widetilde{G}_{n}}\left(\operatorname{Ind}_{H_{1}}^{\widetilde{G}_{n}}\left(\xi_{1} \delta_{H_{1}}^{1 / 2}\right), \operatorname{Ind}_{H_{2}}^{\widetilde{G}_{n}}\left(\xi_{2} \delta_{H_{2}}^{1 / 2}\right)\right) \\
\quad \leq \sum_{H_{1} g H_{2}} \sum_{m=0}^{\infty} \operatorname{dim} \operatorname{Hom}_{H(g)}\left(\xi_{1} \otimes\left(\xi_{2}^{g}\right)^{\vee}, \frac{\delta_{H(g)}}{\left(\delta_{H_{1}} \delta_{H_{2}}^{g}\right)^{1 / 2}} \otimes S^{m}(V(g))\right),
\end{aligned}
$$

where the sum is over all double cosets $H_{1} g H_{2} \in H_{1} \backslash \widetilde{G}_{n} / H_{2}$, and $H(g)=H_{1} \cap\left(g H_{2} g^{-1}\right)$, $V(g)=\mathfrak{g}_{n} /\left(\mathfrak{h}_{1}+\operatorname{Ad}(g) \mathfrak{h}_{2}\right)$ and $\xi^{g}(h)=\xi\left(g^{-1} h g\right)$ for $h \in H(g)$ and $\xi$ a representation of $H_{2}$. Here $\mathfrak{g}_{n}, \mathfrak{h}_{1}$ and $\mathfrak{h}_{2}$ denote the Lie algebras of $\widetilde{G}_{n}, H_{1}$ and $H_{2}$. Further, $\delta_{H(g)}$ is the modulus character of $H(g)$. In our case $H_{1}=\widetilde{G}_{k}^{\triangle} U_{k}, H_{2}=\widetilde{B}_{n}$ and $\xi_{1}=\gamma_{\psi^{\prime}} \otimes \psi, \xi_{2}=\sigma \otimes e^{\nu}, \delta_{H_{1}}=1$, $\delta_{H_{2}}=e^{2 \rho}$. Further, the double coset space $\widetilde{G}_{k}^{\triangle} U_{k} \backslash \widetilde{G}_{n} / \widetilde{B}_{n}$ is parametrized by $\operatorname{diag}\left(S_{k}\right) \backslash S_{n}$, where $S_{n}$ is the symmetric group in $n$ letters and $\operatorname{diag}\left(S_{k}\right) \subset S_{n}$ the diagonal embedding of the symmetric group $S_{k}$ acting on the first and last $k$ letters in the same fashion. For such a permutation matrix $g$ we write $g=\left(\delta_{i, j_{i}}\right)_{i=1, \ldots, n}$, then $g e_{j_{i}}=e_{i}$.

For a permutation matrix $g \in G_{n}$ and $m \geq 0$ we put

$$
\mathcal{H}(g, m)=\operatorname{Hom}_{H(g)}\left(\left(\gamma_{\psi^{\prime}} \otimes \psi\right) \otimes\left(\left(\sigma^{g}\right)^{\vee} \otimes e^{-g(\nu-\rho)}\right), \delta_{H(g)} \otimes S^{m}(V(g))\right),
$$

the Hom-space in (3.37). We claim that $\mathcal{H}(g, m)=0$ unless $m=0$ and

$$
g=g_{0}=\left(\begin{array}{cc} 
& g_{1} \\
g_{2} &
\end{array}\right), \quad \text { where } \quad\left(g_{2}^{-1} g_{1}\right) e_{i}=e_{k-i+1}, i=1, \ldots, k .
$$

Note that $H_{1} g_{0} H_{2}$ is the same equivalence class for all choices of $g_{1}$ and $g_{2}$. Further, for this particular $g=g_{0}$ we have $\mathcal{H}\left(g_{0}, 0\right)=0$ unless $\nu_{i}+\nu_{n-i+1}=0$ for all $i=1, \ldots, k$, and in this case $\operatorname{dim} \mathcal{H}\left(g_{0}, 0\right)=1$. We prove these claims in 3 steps.

(1) First, the same analysis as in the proof of Theorem 3.10 shows that for $m=0$ we have $\mathcal{H}(g, 0)=0$ for $g \neq g_{0}$.

(2) Next we claim that whenever $V(g) \neq 0$ then $\mathcal{H}(g, m)=0$ for all $m>0$. The $H(g)$ representation $V(g)=\mathfrak{g}_{n} /\left(\mathfrak{h}_{1}+\operatorname{Ad}(g) \mathfrak{h}_{2}\right)$ is the sum of the equivalence classes of $E_{\ell, m}$ with either $\ell, m \in\{1, \ldots, k\}, \ell, m \in\{k+1, \ldots, n\}$, or $\ell \in\{k+1, \ldots, n\}$ and $m \in\{1, \ldots, k\}$. We claim that if the equivalence class of some $E_{\ell, m}$ in $V(g)$ is nonzero, then there exists a root subgroup of $H(g)$ that acts nilpotently on $E_{\ell, m}$. This root subgroup acts on the left hand side of $\mathcal{H}(g, m)$ either trivially or by the character $\psi$, and on the right hand side it only acts on $S^{m}(V(g))$. Hence, no homomorphism in $\mathcal{H}(g, m)$ can have a component of $E_{\ell, m}$ in its image. This is true for all generators of $V(g)$ and hence $\mathcal{H}(g, m)=0$ for $m>0$.

It remains to show that every nonzero equivalence class $E_{\ell, m}$ in $V(g)$ has a root subgroup in $H(g)$ acting nilpotently on it. First, if $\ell \in\{k+1, \ldots, n\}$ and $m \in\{1, \ldots, k\}$ with $E_{\ell, m}$ nonzero in $V(g)$, then $E_{\ell, m} \notin \operatorname{Ad}(g) \mathfrak{h}_{2}$. Hence $E_{j_{\ell}, j_{m}} \notin \mathfrak{h}_{2}$ which means $j_{\ell}>j_{m}$. But then $E_{j_{m}, j_{\ell}} \in \mathfrak{h}_{2}$ and therefore $E_{m, \ell} \in \operatorname{Ad}(g) \mathfrak{h}_{2}$. Since also $E_{m, \ell} \in \mathfrak{h}_{1}$ we have $E_{m, \ell} \in \mathfrak{h}(g)$, so $H(g)$ contains the root subgroup $N_{m, \ell}$ which acts nilpotently on $E_{\ell, m}$. 
Next, if $\ell, m \in\{1, \ldots, k\}$ with $E_{\ell, m}$ non-trivial in $V(g)$, then $E_{\ell, m} \notin \operatorname{Ad}(g) \mathfrak{h}_{2}$ and also $E_{\ell+k, m+k} \notin \operatorname{Ad}(g) \mathfrak{h}_{2}$, because $E_{\ell, k}+E_{\ell+k, m+k} \in \mathfrak{h}_{1}$. Hence $E_{j_{\ell}, j_{m}}, E_{j_{\ell+k}, j_{m+k}} \notin \mathfrak{h}$. As before this implies $E_{j_{m}, j_{\ell}}, E_{j_{m+k}, j_{\ell+k}} \in \mathfrak{h}_{2}$ and hence $E_{m, \ell}, E_{m+k, \ell+k} \in \operatorname{Ad}(g) \mathfrak{h}_{2}$. Therefore $E_{m+\ell}+E_{m+k, \ell+k} \in \mathfrak{h}(g)$ and the corresponding root subgroup acts nilpotently on $E_{\ell, m}$. We note that in this case $\ell \neq m$. This shows the claim.

(3) Finally we show that $\mathcal{H}\left(g_{0}, 0\right)$ is non-trivial if and only if $\nu_{i}+\nu_{n-i+1}=0$ for all $i=$ $1, \ldots, k$, and in this case $\operatorname{dim} \mathcal{H}\left(g_{0}, 0\right)=1$. For $g=g_{0}$ we have $H(g)=\left(\widetilde{\mathcal{M}_{k} A_{k}}\right)^{\triangle}$ which is the direct product of $A_{k}^{\triangle}$ and

$$
\widetilde{\mathcal{M}}_{k}^{\triangle}=\left\{ \pm e_{i_{1}} e_{i_{1}+k} \cdots e_{i_{r}} e_{i_{r}+k}: 1 \leq i_{1}<\ldots<i_{r} \leq k, r \in\{0, \ldots, k\}\right\} .
$$

Hence $\delta_{H(g)}=1,\left.\psi\right|_{H(g)}=1$ and we have

$$
\begin{aligned}
\mathcal{H}(g, 0) & =\operatorname{Hom}_{H(g)}\left(\gamma_{\psi^{\prime}} \otimes\left(\sigma^{g}\right)^{\vee} \otimes e^{-g(\nu-\rho)}, \mathbb{C}\right) \\
& =\operatorname{Hom}_{\widetilde{\mathcal{M}}_{k}^{\Delta}}\left(\gamma_{\psi^{\prime}}, \sigma^{g}\right) \otimes \operatorname{Hom}_{A_{k}^{\triangle}}\left(e^{-g(\nu-\rho)}, \mathbb{C}\right) .
\end{aligned}
$$

The second factor is nonzero if and only if $\nu_{i}+\nu_{n-i+1}=\rho_{i}+\rho_{n-i+1}=0$ for all $i=1, \ldots, k$, in which case it is one-dimensional. It remains to show that the first factor is onedimensional. For this we use some Clifford analysis.

The $k$-dimensional subspace $X \subset \mathbb{C}^{n}$ spanned by $f_{i}=e_{i}+\sqrt{-1} e_{i+k}, i=1, \ldots, k$, is isotropic with respect to the standard bilinear form on $\mathbb{C}^{n}$. Let $X^{\prime} \subset \mathbb{C}^{n}$ be the subspace spanned by $f_{i}^{\prime}=e_{i}-\sqrt{-1} e_{i+k}, i=1, \ldots, k$, then $\mathbb{C}^{n}=X \oplus X^{\prime}$. There is an algebra isomorphism $\Phi: C(n) \rightarrow \operatorname{End}\left(\Lambda^{*} X\right), x \mapsto \Phi_{x}$ which is on $X, X^{\prime} \subset C(n)$ given by

$$
\begin{array}{ll}
\Phi_{x}\left(x_{1} \wedge \cdots \wedge x_{r}\right)=x \wedge x_{1} \wedge \cdots \wedge x_{r}, & x \in X, \\
\Phi_{x^{\prime}}\left(x_{1} \wedge \cdots \wedge x_{r}\right)=2 \sum_{i=1}^{r}(-1)^{i-1}\left(x^{\prime} \mid x_{i}\right) x_{1} \wedge \cdots \wedge \widehat{x}_{i} \wedge \cdots \wedge x_{r}, & x^{\prime} \in X^{\prime},
\end{array}
$$

i.e. $X$ acts by exterior multiplication and $X^{\prime}$ by contractions. The pin-representation $\sigma$ of $\widetilde{\mathcal{M}}_{n}$ on $\Lambda^{*} X$ is given by $\sigma(x)=\Phi_{x}$. We compute the action of $\widetilde{\mathcal{M}}_{k}^{\triangle}$ on $\Lambda^{*} X$. For this we use the basis of $\Lambda^{*} X$ given by $f_{I}=f_{i_{1}} \wedge \ldots \wedge f_{i_{r}}$ with $I=\left\{i_{1}, \ldots, i_{r}\right\} \subset\{1, \ldots, k\}$, $1 \leq i_{1}<\ldots<i_{r} \leq k$. First, each monomial $e_{i} e_{i+k}$ acts on the basis elements as by

$$
\begin{aligned}
\sigma\left(e_{i} e_{i+k}\right)\left(f_{i_{1}} \wedge \ldots f_{i_{r}}\right) & =\frac{1}{4 \sqrt{-1}} \sigma\left(f_{i}^{\prime} f_{i}-f_{i} f_{i}^{\prime}\right)\left(f_{I}\right)=\frac{1}{4 \sqrt{-1}}\left(\Phi_{f_{i}^{\prime}} \Phi_{f_{i}}-\Phi_{f_{i}} \Phi_{f_{i}^{\prime}}\right)\left(f_{I}\right) \\
& =\frac{1}{4 \sqrt{-1}}\left(2 \Phi_{f_{i}^{\prime}} \Phi_{f_{i}}-\Phi_{f_{i}^{\prime} f_{i}+f_{i} f_{i}^{\prime}}\right)\left(f_{I}\right)=\frac{1}{4 \sqrt{-1}}\left(2 \Phi_{f_{i}^{\prime}} \Phi_{f_{i}}-4\right)\left(f_{I}\right) \\
& =\frac{1}{\sqrt{-1}} f_{I} \times \begin{cases}+1 & \text { if } i \notin I, \\
-1 & \text { if } i \in I .\end{cases}
\end{aligned}
$$

Hence,

$$
\sigma\left( \pm e_{i_{1}} e_{i_{1}+k} \cdots e_{i_{r}} e_{i_{r}+k}\right) f_{I}= \pm(-\sqrt{-1})^{r}(-1)^{\#\left(I \cap\left\{i_{1}, \ldots, i_{r}\right\}\right)} .
$$

Note that $\left.\sigma^{g}\right|_{\widetilde{\mathcal{M}}_{k}^{\Delta}}=\left.\sigma\right|_{\widetilde{\mathcal{M}}_{k}^{\Delta}}$. To determine the action of $\widetilde{\mathcal{M}}_{k}^{\triangle}$ under $\gamma_{\psi^{\prime}}$ we assume that $\gamma_{\psi^{\prime}}(-1)=\sqrt{-1}^{k}$. The case of $\gamma_{\psi^{\prime}}(-1)=-\sqrt{-1}$ is handled similarly. Then

$$
\begin{aligned}
\gamma_{\psi^{\prime}}\left( \pm e_{i_{1}} e_{i_{1}+k} \cdots e_{i_{r}} e_{i_{r}+k}\right) & = \pm(-1)^{r+\frac{r(r-1)}{2}} \gamma_{\psi^{\prime}}\left(e_{i_{r}+k} \cdots e_{i_{1}+k} e_{i_{r}} \cdots e_{i_{1}}\right) \\
& = \pm(-1)^{r+\frac{r(r-1)}{2}} \gamma_{\psi^{\prime}}\left(\mathfrak{s}\left(\varepsilon_{I \cup(I+k)}\right)\right.
\end{aligned}
$$




$$
\begin{aligned}
& = \pm(-1)^{r+\frac{r(r-1)}{2}} \gamma_{\psi^{\prime}}\left((-1)^{r}\right) \\
& = \pm(-\sqrt{-1})^{r} .
\end{aligned}
$$

Hence, an intertwining operator in $\operatorname{Hom}_{\widetilde{\mathcal{M}}_{k}^{\Delta}}\left(\gamma_{\psi^{\prime}}, \sigma^{g}\right)$ can only map onto $f_{I}$ for the empty set $I$, so this space is one-dimensional.

\section{Metaplectic Shalika model for exceptional Representations}

4.1. Exceptional representations. The exceptional representations were introduced and studied by Kazhdan and Patterson [KP84, locally and globally. Over a local field, the exceptional representation $\theta_{n}$ of $\widetilde{G}_{n}$ is the unique irreducible quotient of a principal series representation corresponding to a genuine character, which is a lift of $\left.\delta_{B_{n}}^{1 / 4}\right|_{T_{n}^{2}}$ to $C_{\widetilde{T}_{n}}$ (in the notation of $\S 2.2 \theta$ is a quotient of $\mathrm{I}(\chi)$ where $\left.\left.\chi\right|_{T_{n}^{2}}=\delta_{B_{n}}^{1 / 4}\right)$. When $n$ is odd, there are $\left[F^{*}: F^{* 2}\right]$ possible lifts of $\left.\delta_{B_{n}}^{1 / 4}\right|_{T_{n}^{2}}$ to $C_{\widetilde{T}_{n}}$, resulting in non-isomorphic representations, but $\theta_{2 k}$ is unique.

Any exceptional representation of $\widetilde{G}_{n}$ can be obtained by twisting $\theta_{n}$ with a character of $F^{*}$ (pulled back to $G_{n}$ via det). Here for simplicity we take this character to be trivial.

Over a global field we denote this representation by $\Theta_{n}$, it has an automorphic realization as the residual representation of an Eisenstein series on $B_{n}(F) \backslash G_{n}(F)$. Abstractly, it is isomorphic to the restricted tensor product of local exceptional representations. For a detailed description of the properties of exceptional representations see [KP84, BG92, Kab01].

Remark 4.1. Over $\mathbb{C}$ the group $G_{n}$ splits under the cover, then the results of Vogan [DAV86, Lemma 2.4, Corollary 11.6 and Lemma 11.11] show that $\theta_{2 k}$ is isomorphic to the Stein complementary series representation $\operatorname{Ind}_{Q_{k}}^{G_{2 k}}\left(\delta_{Q_{k}}^{1 / 2+1 /(2 k)}\right) \cong \operatorname{Ind}_{Q_{k}}^{G_{2 k}}\left(\delta_{Q_{k}}^{1 / 2-1 /(2 k)}\right)$. In particular it is unitary, its $K$-types have multiplicity one, and it contains the trivial $K$-type. In the real case this is not true since $\theta_{2 k}$ is genuine and $\widetilde{Q}_{k}$ does not have any genuine characters to induce from.

4.2. Local metaplectic Shalika model. Let $F$ be a $p$-adic field, $n=2 k$ and $\theta=\theta_{n}$. In Kap15a, Theorem 3.1] we proved that $\theta_{U_{k}, \psi}$ is the one-dimensional representation of $\widetilde{G}_{k}^{\triangle}$ given by

$$
\epsilon \mathfrak{s}\left(c^{\triangle}\right) \rightarrow \epsilon \gamma_{\psi,(-1)^{k}}(\operatorname{det} c), \quad \epsilon \in \mu_{2} .
$$

In particular $\theta$ admits a unique (up to scalar multiplication) metaplectic $\left(\psi_{(-1)^{k}}, \psi\right)$-Shalika functional and a unique metaplectic Shalika model $\mathscr{S}\left(\theta, \psi_{(-1)^{k}}, \psi\right)$.

Assume all data are unramified as in $\S$ 2.1. Then $\theta$ is also unramified. The unramified normalized function $\mathscr{S} \in \mathscr{S}\left(\theta, \psi_{(-1)^{k}}, \psi\right)$ is the unique function which is identically 1 on $\kappa(K)$.

Lemma 4.2. For any $\lambda \in \mathbb{Z}_{\geq}^{k}$,

$$
\mathscr{S}\left(\mathfrak{s}\left(t_{\lambda}\right)\right)= \begin{cases}\delta_{B_{n}}^{1 / 4}\left(t_{\lambda}\right) & \lambda \in 2 \mathbb{Z}_{+}^{k} \\ 0 & \text { otherwise }\end{cases}
$$

Proof. In the notation of $\S 2.2, \theta$ is a quotient of $\mathrm{I}(\chi)$ where $\chi=\chi_{\underline{s}}=\delta_{B_{n}}^{1 / 4}$,

$$
\underline{s}=\frac{1}{4}(n-1, n-3, \ldots, 3-n, 1-n) .
$$


This character satisfies (3.17). Put $\psi^{\prime}=\psi_{\varrho}$ with $\varrho=(-1)^{k}$. On the one hand let $l_{\theta}$ be the metaplectic $\left(\psi^{\prime}, \psi\right)$-Shalika functional on $\theta$ such that $\mathscr{S}(g)=l_{\theta}\left(\theta(g) \varphi_{K, \chi}\right)$. Clearly it extends to a similar nonzero functional on $\mathrm{I}(\chi)$.

On the other hand let $l$ be the nonzero metaplectic $\left(\psi^{\prime}, \psi\right)$-Shalika functional on $\mathrm{I}(\chi)$, whose existence was guaranteed by Corollary 3.35. By Theorem 3.10 the functionals $l_{\theta}$ and $l$ are proportional, in particular $l$ is nonzero on $\varphi_{K, \chi}$, so that $l_{\theta}=l\left(\varphi_{K, \chi}\right)^{-1} l$ and

$$
\mathscr{S}(g)=l\left(\varphi_{K, \chi}\right)^{-1} l\left(g \varphi_{K, \chi}\right), \quad \forall g \in \widetilde{G}_{n} .
$$

Looking at the formula of Theorem 3.28 for $\mathrm{I}(\chi)$ and $\varphi_{K, \chi}$, the coefficient corresponding to $w \in W_{\mathrm{Sp}_{k}}$ takes the form

$$
Q^{-1} \prod_{\{\alpha>0: w \alpha>0\}} c_{\alpha}(\chi) \prod_{\left\{\alpha \in \Sigma_{\mathrm{Sp}_{k}}^{+}: w \alpha<0\right\}} y_{\alpha}(\chi) .
$$

Observe that none of the terms here has a pole, and by definition for any $\alpha \in \Delta_{G_{n}}$ (a simple root), $\chi\left(a_{\alpha}\right)=q^{-1}$ so that $c_{\alpha}\left(\chi^{-1}\right)=0$. Now consider the product of factors $y_{\alpha}(\chi)$. If $w \alpha<0$ for some short root $\alpha \in \Sigma_{\mathrm{Sp}_{k}}^{+}, y_{\alpha}(\chi)=c_{\alpha}(\chi) c_{\alpha}\left(\chi^{-1}\right)=0$. Otherwise if $w \neq e$, we must have $w \alpha_{k}<0$. But then since

$$
\begin{gathered}
\epsilon_{\varrho, k}=\left(-(-1)^{k}, \varpi\right)_{2}(\varpi, \varpi)_{2}^{k-1}=1, \\
y_{\alpha_{k}}(\chi)=\frac{\left(1+q^{-1} \epsilon_{\varrho, k}\right)\left(1-\epsilon_{\varrho, k}\right)}{1-q}=0 .
\end{gathered}
$$

Therefore all coefficients except the one for $w=e$ vanish and for $\lambda \in 2 \mathbb{Z}_{+}^{k}$,

$$
l\left(\mathfrak{s}\left(t_{\lambda}\right) \varphi_{K, \chi}\right)=Q^{-1} c_{w_{0}}(\chi) \delta_{B_{n}}^{1 / 2} \chi^{-1}\left(\mathfrak{s}\left(t_{\lambda}\right)\right)=Q^{-1} c_{w_{0}}(\chi) \delta_{B_{n}}^{1 / 4}\left(t_{\lambda}\right) .
$$

We conclude $l_{\theta}=Q c_{w_{0}}(\chi)^{-1} l$ and the result immediately follows.

Remark 4.3. The proof implies that $l$ factors through the quotient $\theta$, which is also the image of the intertwining operator $T_{w_{0}}$. Since by (2.6), $T_{w_{0}} \varphi_{K, \chi}=c_{w_{0}}(\chi) \varphi_{K, \chi^{-1}}$, it is natural to find $c_{w_{0}}(\chi)$ in the normalization.

Remark 4.4. One could attempt to consider $\theta$ as a subrepresentation of $\mathrm{I}\left(\chi^{-1}\right)$. As such, it contains $\varphi_{K, \chi^{-1}}$ and the formula of Theorem 3.28 for $\left(\mathrm{I}\left(\chi^{-1}\right), \varphi_{K, \chi^{-1}}\right)$ may be used. However unless $k=1$, all the coefficients, which now take the form

$$
Q^{-1} \prod_{\{\alpha>0: w \alpha>0\}} c_{\alpha}\left(\chi^{-1}\right) \prod_{\left\{\alpha \in \Sigma_{\mathrm{Sp}_{k}}^{+}: w \alpha<0\right\}} y_{\alpha}\left(\chi^{-1}\right),
$$

vanish (if $w=w_{0}$ and $k>1, y_{\alpha_{1}}\left(\chi^{-1}\right)=0$ ). In other words, the functional $\Lambda_{\chi}$ vanishes on $\theta$. This is the phenomena described in Remark 3.34. This also means that the metaplectic Shalika functional on $\theta$ does not extend to $\mathrm{I}\left(\chi^{-1}\right)$.

Now assume $F=\mathbb{R}$. The representation $\theta$ is the unique irreducible quotient of a principal series representation, which satisfies the assumptions of Theorem 3.37. Hence the space of metaplectic Shalika functionals on $\theta$ is at most one-dimensional. This remains true over $\mathbb{C}$, because then the cover is split over $G_{n}$ and since $\theta$ is an irreducible admissible smooth Fréchet representation, the bound follows from AGJ09. We will prove that the space of metaplectic Shalika functionals on $\theta$ is precisely one-dimensional over both $\mathbb{R}$ and $\mathbb{C}$ : existence of the functional will follow from a local-global argument in $\S 4.3$ (Corollary 4.25). 
4.3. Fourier coefficients of exceptional representations. Let $F$ be a global field and $\Theta=\Theta_{n}$. For an automorphic form $\varphi$ in the space of $\Theta$, a unipotent subgroup $U$, and a character $\psi$ of $U(\mathbb{A})$ which is trivial on $U(F)$, let $\varphi^{U, \psi}$ be the corresponding Fourier coefficient, given by

$$
\varphi^{U, \psi}(g)=\int_{U(F) \backslash U(\mathbb{A})} \varphi(u g) \psi^{-1}(u) d u, \quad g \in \widetilde{G}_{n}(\mathbb{A}) .
$$

These coefficients are related to local twisted Jacquet modules at the finite places. More explicitly, if a Fourier coefficient does not vanish identically on $\Theta$, then for each place $v<\infty$ of $F,\left(\Theta_{v}\right)_{U, \psi} \neq 0$. Conversely if $\left(\Theta_{v}\right)_{U, \psi}=0$ for some $v<\infty$, the coefficient (4.1) is zero for any $\varphi$ and $g$ (see e.g., [JR92, Proposition 1]).

Let $\theta=\Theta_{v}$ for some $v<\infty$. In Kap15a we described the twisted Jacquet modules of $\theta$ along the unipotent subgroups $U_{k}$. We use these results to deduce several properties of the Fourier coefficients.

For $0<k<n, M_{k}<G_{n}$ acts on the set of characters of $U_{k}$, with $\min (k, n-k)+1$ orbits. We choose representatives

$$
\psi_{j}(u)=\psi\left(\sum_{i=1}^{j} u_{k-i+1, k+j-i+1}\right), \quad 0 \leq j \leq \min (k, n-k) .
$$

(Here $u$ is regarded as an $n \times n$ matrix.) In particular $\psi_{0}$ is trivial and when $n=2 k, \psi_{k}$ is the Shalika character. For $n=5$ and $k=3$, say, $\psi_{1}(u)=\psi\left(u_{3,4}\right)$ and $\psi_{2}(u)=\psi\left(u_{3,5}+u_{2,4}\right)$. The stabilizer of $\psi_{j}$ in $M_{k}$ is

$$
\mathrm{St}_{n, k}\left(\psi_{j}\right)=\left\{\left(\begin{array}{cccc}
b & v & & \\
& c & & \\
& & c & y \\
& & & d
\end{array}\right): b \in G_{k-j}, c \in G_{j}, d \in G_{n-k-j}\right\} .
$$

Let $V_{j}$ and $Y_{j}$ be the unipotent subgroups of $\operatorname{St}_{n, k}\left(\psi_{j}\right)$ defined by the coordinates of $v$ and $y$. Note that $V_{0}$ and $Y_{0}$ are trivial.

The Jacquet module $\theta_{U_{k}, \psi_{j}}$ is a representation of $p^{-1}\left(\operatorname{St}_{n, k}\left(\psi_{j}\right)\right)$. The following theorem was proved in Kap15a (with minor notational differences):

Theorem 4.5. For any $0 \leq j \leq \min (k, n-k)$, the representation $\theta_{U_{k}, \psi_{j}}$ is isomorphic to $\delta^{1 / 4}\left(\theta_{k-j} \widetilde{\otimes} \theta_{n-k-j}\right) \otimes \gamma_{\psi,(-1)^{j}} \psi_{j}$. Here $\delta$ is the modulus character of the standard parabolic subgroup of $G_{n}$ corresponding to the partition $(k-j, 2 j, n-k-j) ; \widetilde{\otimes}$ is the metaplectic tensor of Kable [Kab01]; $\gamma_{\psi,(-1)^{j}}$ is a genuine character of $\widetilde{G}_{j}^{\triangle}$ and $\gamma_{\psi,(-1)^{j}} \psi_{j}$ is a one-dimensional representation of $\widetilde{G}_{j}^{\triangle} \ltimes U_{j}$, where $G_{j}^{\triangle} \ltimes U_{j}$ is regarded as a subgroup of the middle block of the partition $\left(G_{j}^{\triangle}\right.$ is identified with the coordinates of $\left.c\right)$.

This theorem implies in particular that $V_{j}\left(F_{v}\right)$ and $Y_{j}\left(F_{v}\right)$ act trivially on $\theta_{U_{k}, \psi_{j}}$, and when $n=2 k=2 j, \theta$ admits a unique metaplectic $\left(\psi_{(-1)^{k}}, \psi\right)$-Shalika model (as mentioned in $\S 4.2$. The case $j=0$ of Theorem 4.5 was already proved by Kable [Kab01, Theorem 5.1], in this case $\delta=\delta_{Q_{k}}$ and the result can be used to read off the exponents of $\theta$ (see the proof of Theorem 5.1 in $\S 5.1$.

Proposition 4.6. For any $\varphi$ in the space of $\Theta, 0<k<n$ and $0 \leq j \leq \min (k, n-k), \varphi^{U_{k}, \psi_{j}}$ is trivial on $V_{j}(\mathbb{A})$ and $Y_{j}(\mathbb{A})$. 
Proof. Write the Fourier expansion of $\varphi^{U_{k}, \psi_{j}}$ along $V_{j}(F) \backslash V_{j}(\mathbb{A})$. Any coefficient with respect to a nontrivial character vanishes, because by virtue of Theorem 4.5, at any $v<\infty, V_{j}\left(F_{v}\right)$ acts trivially on $\left(\Theta_{v}\right)_{U_{k}, \psi_{j}}$.

The next theorem describes the constant term of automorphic forms in the space of $\Theta$, along any unipotent radical of a standard parabolic subgroup. The constant term defines an automorphic representation of the Levi part, and as such, it is often convenient to identify it with a tensor representation. Unfortunately, this is not simple to do for covering groups of $G_{n}$, because the preimages in the cover, of the direct factors of the Levi part, do not commute (see e.g., [BG92, Kab01, Tak14]). To avoid this problem, at least to some extent, we define exceptional representations of Levi subgroups. This approach was suggested by Bump and Ginzburg [BG92, § 1], and was perhaps implicit in [Kab01, § 5]. Let $\Delta \subset \Delta_{G_{n}}$ and $Q=Q_{\Delta}$ be the corresponding standard parabolic subgroup, $Q=M \ltimes U$. Let $B_{M}<M$ be the Borel subgroup such that $B_{n}=B_{M} \ltimes U, B_{M}=T_{n} \ltimes N_{M}$. Denote by $\delta_{B_{M}}$ the modulus character of $B_{M}$, as a subgroup of $M$. Also let $W_{M}$ be the Weyl group of $M$ and $w_{M}$ its longest element.

Consider a local context first. A genuine character $\chi$ of $C_{\widetilde{T}_{n}}$ is called $M$-exceptional if $\chi\left(a_{\alpha}\right)=|q|$ for all $\alpha \in \Delta\left(a_{\alpha}\right.$ was defined after (2.5) $)$. The representation $\operatorname{Ind}_{\widetilde{B}_{M}}^{\widetilde{M}}\left(\delta_{B_{M}}^{1 / 2} \chi^{\prime}\right)$, where $\chi^{\prime}$ is an irreducible genuine representation of $\widetilde{T}_{n}$ corresponding to $\chi$, has a unique irreducible quotient called an exceptional representation of $\widetilde{M}$. This follows from the Langlands Quotient Theorem because $\chi$ belongs to the positive Weyl chamber in $W_{M}$. Note that the Langlands Quotient Theorem was proved for covering groups over $p$-adic fields by Ban and Jantzen [BJ13, and over Archimedean fields the proof of Borel and Wallach [BW80] is applicable to covering groups. The exceptional representation of $\widetilde{M}$ is isomorphic to the image of the intertwining operator with respect to $w_{M}$ (over Archimedean fields this follows from [BW80], over $p$-adic fields one can argue as in [KP84, Theorem I.2.9]).

Globally, we construct exceptional representations as residual representations of Eisenstein series. The discussion in [KP84, $\S$ II] (see also [BFG03, § 3]) can be extended to Levi subgroups. For $\underline{s}=\left(s_{1}, \ldots, s_{n}\right) \in \mathbb{C}^{n}$, put

$$
\chi_{\underline{s}}\left(\operatorname{diag}\left(t_{1}, \ldots, t_{n}\right)\right)=\prod_{i=1}^{n}\left|t_{i}\right|^{s_{i}}
$$

We extend $\chi_{\underline{s}}$ to a right $K$-invariant function on $M(\mathbb{A})$ using the Iwasawa decomposition, and lift it to a function on the cover. Let $f$ be an element of $\operatorname{Ind}_{\widetilde{B}_{M}(\mathbb{A})}^{\widetilde{M}(\mathbb{A})}\left(\delta_{B_{M}}^{1 / 2} \chi^{\prime}\right)$, where $\chi^{\prime}$ corresponds to a global $M$-exceptional character $\chi$ (defined similarly to the local one). Denote by $f_{\underline{s}}$ the standard section given by $f_{\underline{s}}(m)=\chi_{\underline{s}}(m) f(m), m \in \widetilde{M}(\mathbb{A})$. Form the Eisenstein series

$$
E_{B_{M}}(m ; f, \underline{s})=\sum_{m_{0} \in B_{M}(F) \backslash M(F)} f_{\underline{s}}\left(m_{0} m\right), \quad m \in \widetilde{M}(\mathbb{A}) .
$$

Identify $\Sigma_{G_{n}}$ with the pairs $(i, j), 1 \leq i \neq j \leq n$, and $\Delta_{G_{n}}$ with the pairs $(i, i+1), 1 \leq i<n$. For $\alpha=(i, j) \in \Sigma_{G_{n}}$, put $\underline{s}_{\alpha}=s_{i}-s_{j}$. The global exceptional representation is spanned by the functions

$$
m \mapsto \operatorname{Res}_{\underline{s}=\underline{0}} E_{B_{M}}(m ; f, \underline{s})=\lim _{\underline{s} \rightarrow \underline{0}} \prod_{\alpha \in \Delta} \underline{s}_{\alpha} E_{B_{M}}(m ; f, \underline{s}) .
$$


It is isomorphic to the restricted direct product of local exceptional representations. The proof is analogous to [KP84, Proposition II.1.2 and Theorems II.1.4 and II.2.1] and [BFG03, Proposition 3.1 and Theorem 3.2].

Let $\Theta_{M}$ (resp., $\theta_{M, v}$ ) be the global (resp., local over $F_{v}$ ) exceptional representation corresponding to a genuine character, which is a lift of $\left.\delta_{B_{M}}^{1 / 4}\right|_{T_{n}^{2}}$ to $C_{\widetilde{T}_{n}}$. Then $\Theta_{M}=\otimes_{v}^{\prime} \Theta_{M, v}$. In particular $\Theta_{G_{n}}=\Theta$.

Remark 4.7. Over a p-adic field, the aforementioned result [Kab01, Theorem 5.1] also implies that an exceptional representation of $\widetilde{M}$ is isomorphic to a metaplectic tensor of exceptional representations of the preimages of the direct factors of $M$.

We are ready to state the result regarding the constant term of elements of $\Theta$.

Theorem 4.8. Let $\varphi$ belong to the space of $\Theta$. The function $m \mapsto \varphi^{U}(m)$ on $\widetilde{M}(\mathbb{A})$ belongs to the space of $\delta_{Q}^{1 / 4} \Theta_{M}$.

Remark 4.9. This was proved in [KP84] for $Q=B_{n}$.

Remark 4.10. If $F$ is a function field, this result follows immediately from the computation of the Jacquet modules of $\theta$ over p-adic fields in [Kab01, Theorem 5.1].

Remark 4.11. A priori, the constant term does not vanish identically, because it does not vanish for $N_{n}$ (this follows from the construction of the exceptional representation in KP84, $\S \mathrm{II}])$.

Remark 4.12. Similar results were obtained in $[\mathrm{Kap} 15 \mathrm{~b}]$ for the small representation of a cover of $\mathrm{SO}_{2 n+1}$ (in the sense of [BFG03]), and in [Kap16] for the analog of this representation for $\mathrm{GSpin}_{2 n+1}$. In the case of $\mathrm{SO}_{2 n+1}$, the direct factors of maximal parabolic subgroups do commute in the cover, so that a more precise result could be obtained. For GSpin $2 n+1$ the direct factors commute only for the maximal parabolic subgroup obtained by removing the unique short simple root.

Proof. As explained above and keeping the same notation (with $M=G_{n}$ ), we can assume

$$
\varphi(g)=\operatorname{Res}_{\underline{s}=\underline{0}} E_{B_{n}}(g ; f, \underline{s}),
$$

for some $f$ in the space of $\operatorname{Ind}_{\widetilde{B}_{n}(\mathbb{A})}^{\widetilde{G}_{n}(\mathbb{A})}\left(\delta_{B_{n}}^{1 / 2} \chi^{\prime}\right)$, where $\chi^{\prime}$ corresponds to a genuine lift of $\left.\delta_{B_{n}}^{1 / 4}\right|_{T_{n}^{2}}$.

Let $\mathcal{W} \subset W$ be with $G_{n}=\coprod_{w \in \mathcal{W}} B_{n} w^{-1} Q$. For $X<Q$ and $w \in \mathcal{W}$, set $X^{w}={ }^{w} B_{n} \cap X$. Define

$$
\begin{aligned}
& f_{\underline{s}, w}(m)=M\left(w, \chi_{\underline{s}}\right) f_{\underline{s}}(m)=\int_{U^{w}(F) \backslash U(\mathbb{A})} f_{\underline{s}}\left(w^{-1} u m\right) d u, \\
& E_{M^{w}}\left(f_{\underline{s}, w}(m)\right)=\sum_{m_{0} \in M^{w}(F) \backslash M(F)} f_{\underline{s}, w}\left(m_{0} m\right) .
\end{aligned}
$$

Here $m \in \widetilde{M}(\mathbb{A})$ (although the definition is valid also on $\widetilde{G}_{n}(\mathbb{A})$ ). The intertwining operator $M\left(w, \chi_{\underline{s}}\right)$ is in fact defined by the meromorphic continuation of this integral. According to Mœglin and Waldspurger [MW95, § II.1.7],

$$
\varphi^{U}(m)=\sum_{w \in \mathcal{W}} \operatorname{Res}_{\underline{s}=\underline{0}} E_{M^{w}}\left(f_{\underline{s}, w}(m)\right) .
$$


We can take the representatives $\mathcal{W}$ such that for all $w \in \mathcal{W}$,

$$
N_{M}<{ }^{w} N_{n} \text {. }
$$

Indeed this follows from [BZ77, 2.11] (applied to $W^{T_{n}, M}$ in their notation, $\left.{ }^{w^{-1}}\left(M \cap B_{n}\right)<B_{n}\right)$.

Claim 4.13. $f_{\underline{s}, w}$ is holomorphic at $\underline{s} \rightarrow \underline{0}$, except for simple poles in the variables $\underline{s}_{\alpha}$ with $\alpha \in \Delta_{G_{n}}$ such that $w \alpha<0$.

Claim 4.14. $E_{M^{w}}\left(f_{\underline{s}, w}(m)\right)$ is holomorphic at $\underline{s} \rightarrow \underline{0}$, except for at most $\left|\Delta_{G_{n}}\right|$ simple poles. There are less than $\left|\vec{\Delta}_{G_{n}}\right|$ poles unless $w=w_{M} w_{0}$, in which case $m \mapsto E_{M^{w}}\left(f_{\underline{s}, w}(m)\right)$ belongs to the space of $\Theta_{M}$.

Granted the claims, the result quickly follows: by Claim 4.14, any summand with $w \neq$ $w_{M} w_{0}$ vanishes when we take the residue, and we are left with $E_{M^{w_{M} w_{0}}}\left(f_{\underline{s}, w_{M} w_{0}}(m)\right)$. Since at most one summand is nonzero, as explained in Remark 4.11 this summand is nonzero for some data.

Proof of Claim 4.13. Since $f$ is left-invariant under $N_{n}(\mathbb{A})$ and $U^{w}<N_{n}$, we may rewrite the integration in (4.2) over $U^{w}(\mathbb{A}) \backslash U(\mathbb{A})$. Moreover (4.5) implies

$$
N_{M} U^{w}=N_{M}{ }^{w} B_{n} \cap N_{M} U={ }^{w} B_{n} \cap N_{n}=N_{n}^{w},
$$

hence $U^{w} \backslash U=N_{n}^{w} \backslash N_{n}$ so that we can write the integral in (4.2) over $N_{n}^{w}(\mathbb{A}) \backslash N_{n}(\mathbb{A})$. Now we proceed as in Kap16, Claim 3.5]. We can assume that $f$ is a pure tensor. At any finite place, the local intertwining operator is holomorphic for $\underline{s}$ in a small neighborhood of $\underline{0}$, because then the local component of $\chi^{\prime} \chi_{\underline{s}}$ belongs to the positive Weyl chamber. Thus the poles are global, in the sense that they appear in the product of local intertwining operators applied to the unramified normalized components of $f$. Now we use the Gindikin-Karpelevich formula (2.6) to identify the possible poles.

Let $\alpha \in \Sigma_{G_{n}}^{+}$and assume $w \alpha<0$. Recall the function $c_{\alpha}(\cdots)$ (defined before (2.6) ). The poles occur in the products

$$
\prod_{v} c_{\alpha}\left(\left(\chi^{\prime} \chi_{\underline{s}}\right)_{v}\right)=\frac{\zeta\left(C_{\alpha}+2 \underline{s}_{\alpha}\right)}{\zeta\left(C_{\alpha}+2 \underline{s}_{\alpha}+1\right)},
$$

where $\zeta$ is the partial Dedekind zeta function of $F$ and $0<C_{\alpha} \in \mathbb{Z}$ is an integer depending on $\chi^{\prime}$. As a function of $\underline{s}_{\alpha}$, there is a pole when $\underline{s}_{\alpha} \rightarrow 0$ if and only if $C_{\alpha}=1$, this occurs precisely when $\alpha \in \Delta_{G_{n}}$. We also deduce that the poles are simple.

Proof of Claim 4.14. The poles of $E_{M^{w}}\left(f_{\underline{s}, w}(m)\right)$ are either poles of $f_{\underline{s}, w}(m)$, which were identified in Claim 4.13, or poles incurred by the summation over $M^{w} \backslash M$. Because $U^{w} \backslash U=$ $N_{n}^{w} \backslash N_{n}, f_{\underline{s}, w}$ is an element in the space of

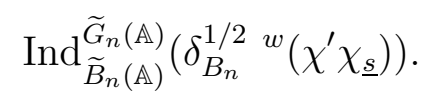

Then the restriction of $f_{\underline{s}, w}$ to $\widetilde{M}(\mathbb{A})$ belongs to

$$
\delta_{Q}^{1 / 2} \operatorname{Ind}_{\widetilde{B}_{M}(\mathbb{A})}^{\widetilde{M}(\mathbb{A})}\left(\delta_{B_{M}}^{1 / 2} w\left(\chi^{\prime} \chi_{\underline{s}}\right)\right) .
$$

Since $N_{M}<{ }^{w} B_{n} \cap M$ (by (4.5) $), B_{M}={ }^{w} B_{n} \cap M$ ( $w$ cannot conjugate more positive roots from $N_{n}$ into $M$, because $N_{M}$ is a maximal unipotent subgroup in $M$ ). Hence (4.3) is a series over $B_{M} \backslash M$. To locate its poles we consider the constant term along $N_{M}$. Arguing as in 
[KP84, Proposition II.1.2], the constant term is a sum of intertwining operators $M\left(\omega, \chi_{\underline{s}}\right) f_{\underline{s}, w}$, where $\omega$ varies over $W_{M}$.

Fix $\omega$ and let $\Sigma_{M}$ (resp., $\Sigma_{M}^{+}$) be the set of roots (resp., positive roots) spanned by the roots in $\Delta$. Assumption (4.5) implies that ${ }^{w}\left(\chi^{\prime} \chi_{\underline{s}}\right)$ belongs to the positive Weyl chamber of $W_{M}$ (when $\underline{s} \rightarrow \underline{0}$ ). Therefore, as in the proof of Claim 4.13 any pole must be located in a quotient of $\zeta$ functions

$$
\prod_{v} c_{\alpha}\left({ }^{w}\left(\chi^{\prime} \chi_{\underline{s}}\right)_{v}\right)=\frac{\zeta\left(C_{w, \alpha}+2 \underline{s}_{w^{-1} \alpha}\right)}{\zeta\left(C_{w, \alpha}+2 \underline{s}_{w^{-1} \alpha}+1\right)} .
$$

Here $\alpha \in \Sigma_{M}^{+}$and $\omega \alpha<0 ; 0<C_{w, \alpha} \in \mathbb{Z}$ depends on ${ }^{w} \chi^{\prime}$, specifically $\left({ }^{w} \chi^{\prime}\right)_{v}\left(a_{\alpha}\right)=q_{v}^{-C_{w, \alpha}}$. The quotient has a pole if and only if $C_{w, \alpha}=1$. Since $C_{\alpha}=1$ if and only if $\alpha \in \Delta_{G_{n}}$,

$$
\left\{\alpha \in \Sigma_{M}^{+}: C_{w, \alpha}=1\right\}=\left\{\alpha \in \Delta_{G_{n}}: w \alpha \in \Sigma_{M}^{+}\right\} .
$$

Thus the number of poles of the series is bounded by the size of (4.7). Since the conditions $w \alpha \in \Sigma_{M}^{+}$and $w \alpha<0$ are disjoint, the number of poles of $E_{M^{w}}\left(f_{\underline{s}, w}(m)\right)$ is at most $\left|\Delta_{G_{n}}\right|$. The poles of $f_{\underline{s}, w}$ are simple, they occur in the variables $\underline{s}_{\beta}$ where $\beta>0$ and $w \beta<0$. A pole of (4.6) is also simple, it occurs in $\underline{s}_{w^{-1} \alpha}$ with $\alpha>0$, and because $w\left(w^{-1} \alpha\right)=\alpha>0$, this pole does not appear in the set of poles of $f_{\underline{s}, w}$. Thus the poles of $E_{M^{w}}\left(f_{\underline{s}, w}(m)\right)$ are simple.

Assuming $\left|\Delta_{G_{n}}\right|$ poles are obtained, we prove $w=w_{M} w_{0}$. In this case we must have

$$
\Delta_{G_{n}}=\left\{\alpha \in \Delta_{G_{n}}: w \alpha<0\right\} \amalg\left\{\alpha \in \Delta_{G_{n}}: w \alpha \in \Sigma_{M}^{+}\right\},
$$

and then (4.5) immediately implies $w=w_{M} w_{0}$. Since in this case ${ }^{w} \delta_{B_{n}}=\delta_{Q}^{-1} \delta_{B_{M}}$, the restriction of $f_{\underline{s}, w}$ to $\widetilde{M}(\mathbb{A})$ belongs to $\delta_{Q}^{1 / 4} \operatorname{Ind}_{\widetilde{B}(\mathbb{A})}^{\widetilde{M}(\mathbb{A})}\left(\delta_{B_{M}}^{1 / 2} \chi^{\prime \prime}\right)$, where $\chi^{\prime \prime}$ corresponds to a lift of $\left.\delta_{B_{M}}^{1 / 4}\right|_{T_{n}^{2}}$, and the definition of $\Theta_{M}$ implies that

$$
m \mapsto \operatorname{Res}_{\underline{s}=\underline{0}} E_{M w}\left(f_{\underline{s}, w}(m)\right)
$$

belongs to the space of $\delta_{Q}^{1 / 4} \Theta_{M}$.

Remark 4.15. Takeda [Tak14, Proposition 2.45] computed the constant term along the unipotent radical corresponding to the partition $(2, \ldots, 2)$, for the twisted exceptional representation (his proof does not apply to the non-twisted version, i.e., to our setting). He developed a generalized Weil representation for the preimage of the Levi part, defined a global block-compatible cocycle and was able to provide an explicit realization, whose properties we shall use below (see the proof of Claim 4.24). However, it is not expected that a similar realization will apply for a general Levi subgroup: the realization of $\theta_{n}$ as essentially a Weil representation is only valid for $n=2$.

Now we describe the Fourier coefficient along the Shalika unipotent subgroup and character. Assume $n=2 k$. The stabilizer of $\psi_{k}$ in $M_{k}$ is now $G_{k}^{\triangle}$.

Claim 4.16. The section $\mathfrak{s}$ is well defined on $G_{k}^{\triangle}(\mathbb{A})$ and satisfies a global relation similar to (3.1), with the Hilbert symbol replaced by the product of local Hilbert symbols at all places.

Proof. If $g \in G_{k}^{\triangle}(\mathbb{A})$, for almost all $v, g_{v} \in G_{k}^{\triangle}\left(\mathcal{O}_{v}\right)$ and then by Claim $3.18, \mathfrak{s}_{v}\left(g_{v}\right)=\kappa_{v}\left(g_{v}\right)$. Hence $\mathfrak{s}$ is well-defined. The global relation follows from the local one, since $\mathfrak{s}=\prod_{v} \mathfrak{s}_{v}$. 
Henceforth we identify $G_{k}^{\triangle}(\mathbb{A})$ with its image in $\widetilde{G}_{n}(\mathbb{A})$ under $\mathfrak{s}$.

Let $\pi$ be any automorphic representation of $\widetilde{G}_{n}(\mathbb{A})$. We say that $l$ is a global metaplectic Shalika functional on $\pi$, if $l$ satisfies the global analog of $(\underline{3.2})$, where $c \in G_{k}(\mathbb{A}), u \in U_{k}(\mathbb{A})$ and $\gamma_{\psi^{\prime}}$ is now the global Weil factor. As in the case of the Whittaker model, we are interested in concrete realizations of such functionals, e.g., given as integrals. A global integral for the Shalika model of $G_{n}$ was studied in [JS90, FJ93], involving an integration over $G_{k}^{\triangle}(\mathbb{A}) \times U_{k}(\mathbb{A})$. For exceptional representations, we will show that $\varphi^{U_{k}, \psi_{k}}$ already satisfies the required properties, i.e., the reductive part of the integration is not needed.

Remark 4.17. Beineke and Bump [BB06] also studied, in the non-metaplectic case, local and global Shalika functionals given solely by unipotent integration, for a specific representation.

To study the properties of $\varphi^{U_{k}, \psi_{k}}$, we first relate it to the Fourier coefficient of $\Theta$ along $N_{n}$ and its degenerate character $\psi^{\circ}$ given by

$$
\psi^{\circ}(v)=\sum_{i=1}^{k} \psi\left((-1)^{k-i} v_{2 i-1,2 i}\right)
$$

(called a semi-Whittaker coefficient in [BG92]).

Define $w \in G_{n}(F)$ as follows. For all $1 \leq i \leq k$, it has 1 on the $(2 i-1, i)$-th coordinate and $(-1)^{k-i}$ on the $(2 i, k+i)$-th coordinate. Its other entries are zero. Let $V<U_{k}$ be the subgroup obtained from $U_{k}$ by zeroing all upper diagonal entries except those on rows $i+1, \ldots, k$ of column $k+i$, for all $1 \leq i \leq k-1$.

Example 4.18. When $k=2$,

$$
w=\left(\begin{array}{cccc}
1 & & & \\
& & -1 & \\
& 1 & & \\
& & & 1
\end{array}\right), \quad V=\left\{\left(\begin{array}{cccc}
1 & & & \\
& 1 & v_{1} & \\
& & 1 & \\
& & & 1
\end{array}\right)\right\}
$$

We prove:

Lemma 4.19. For any $\varphi$ in the space of $\Theta$,

$$
\varphi^{U_{k}, \psi_{k}}(g)=\int_{V(\mathbb{A})} \varphi^{N_{n}, \psi^{\circ}}(w v g) d v .
$$

Proof. For $0 \leq l \leq k-1$, define $U^{(l)}<N_{n}$, a character $\psi^{(l)}$ of $U^{(l)}(F) \backslash U^{(l)}(\mathbb{A}), w^{(l)} \in G_{n}(F)$, and $V^{(l)}<N_{n}$ as follows. Let

$$
\begin{aligned}
& U^{(l)}=\left\{\left(\begin{array}{ccc}
z & x_{1} & x_{2} \\
& I_{k-l} & u \\
& & I_{k-l}
\end{array}\right): z \in N_{2 l}\right\}, \\
& \psi^{(l)}(u)=\sum_{i=1}^{l} \psi\left((-1)^{k-i} z_{2 i-1,2 i}\right) \psi(\operatorname{tr}(u)) .
\end{aligned}
$$

Note that $\psi^{(l)}$ is the product of a degenerate character depending only the odd rows of $z$, and the Shalika character on the coordinates of $u$.

The matrix $w^{(l)}$ : for $1 \leq i \leq l$, has 1 in the $(2 i-1, i)$-coordinate and $(-1)^{k-i}$ in the $(2 i, k+i)$-coordinate, and for $1 \leq i \leq k-l$, has 1 in coordinates $(2 l+i, l+i)$ and $(k+l+$ $i, k+l+i)$, and 0 elsewhere. 
The subgroup $V^{(l)}$ : it is obtained from $N_{n}$ by zeroing all upper diagonal entries except those in rows $i+1, \ldots, k$ of column $k+i$, for all $1 \leq i \leq l$.

Example 4.20. When $k=3$,

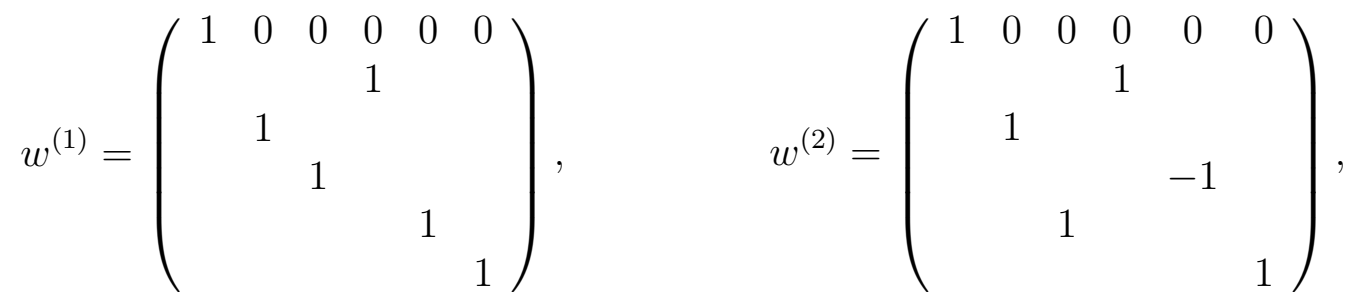

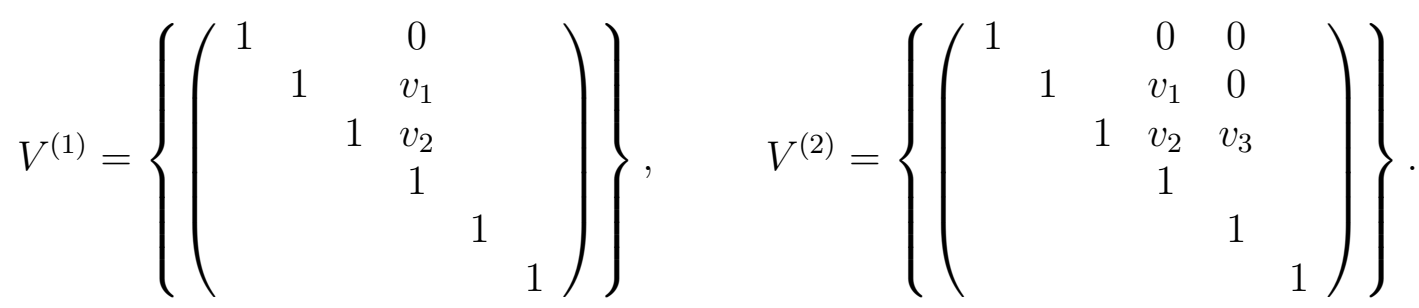

In particular $U^{(0)}=U_{k}, \psi^{(0)}=\psi_{k}$ (the Shalika character of $\left.U_{k}\right), w^{(0)}=I_{n}, V^{(0)}=\left\{I_{n}\right\}$; and $U^{(k-1)}=N_{n}, \psi^{(k-1)}=\psi^{\circ}, w^{(k-1)}=w, V^{(k-1)}=V$.

Claim 4.21. For any $0 \leq l \leq k-2$,

$$
\begin{aligned}
& \int_{V^{(l)}(\mathbb{A})} \int_{U^{(l)}(F) \backslash U^{(l)}(\mathbb{A})} \varphi\left(u w^{(l)} v\right)\left(\psi^{-1}\right)^{(l)}(u) d u d v \\
& =\int_{V^{(l+1)}(\mathbb{A})} \int_{U^{(l+1)}(F) \backslash U^{(l+1)}(\mathbb{A})} \varphi\left(u w^{(l+1)} v\right)\left(\psi^{-1}\right)^{(l+1)}(u) d u d v .
\end{aligned}
$$

The lemma follows from the repeated application of this claim.

Proof of Claim 4.21. We apply the technique of "exchange of roots", following Ginzburg Gin90] (see also [GRS01, Sou05, GRS11]). Identify $U_{k-l}$ with the subgroup of $U^{(l)}$ defined by the coordinates of $u$. Denote an element of $U_{k-l}$ by

$$
u\left(u_{1}, u_{2}, u_{3}, u_{4}\right)=\left(\begin{array}{cccc}
1 & & u_{1} & u_{2} \\
& I_{k-l-1} & u_{3} & u_{4} \\
& & 1 & \\
& & & I_{k-l-1}
\end{array}\right) .
$$

By definition the restriction of $\psi^{(l)}$ to $U_{k-l}$ is given by $\psi\left(u_{1}\right) \psi\left(\operatorname{tr}\left(u_{4}\right)\right)$. Set

$$
\begin{aligned}
& Y=\left\{u\left(0,0, u_{3}, 0\right)\right\}<U_{k-l}, \quad C=\left\{u\left(u_{1}, u_{2}, 0, u_{4}\right)\right\}<U_{k-l}, \\
& X=\left\{\left(\begin{array}{ccc}
1 & e & \\
& I_{k-l-1} & \\
& & I_{k-l}
\end{array}\right)\right\} .
\end{aligned}
$$

Let $U^{\prime}<U^{(l)}$ be defined by the coordinates of $z, x_{1}$ and $x_{2}$, and define the following function on $\widetilde{G}_{n}(\mathbb{A})$,

$$
\varphi^{\prime}(g)=\int_{U^{\prime}(F) \backslash U^{\prime}(\mathbb{A})} \varphi\left(u^{\prime} g\right)\left(\psi^{-1}\right)^{(l)}\left(u^{\prime}\right) d u^{\prime} .
$$


In particular, $\varphi^{\prime}$ is trivial on $U_{k-l}(F)$. Since $U^{(l)}=U^{\prime} \rtimes U_{k-l}$, the left-hand side of (4.8) becomes

$$
\int_{V^{(l)}(\mathbb{A})} \int_{U_{k-l}(F) \backslash U_{k-l}(\mathbb{A})} \varphi^{\prime}\left(u w^{(l)} v\right)\left(\psi^{-1}\right)^{(l)}(u) d u d v .
$$

According to Ginzburg, Rallis and Soudry GRS11] (Lemma 7.1, with $X, Y$ and $C$ as above),

$$
\int_{U_{k-l}(F) \backslash U_{k-l}(\mathbb{A})} \varphi^{\prime}(u)\left(\psi^{-1}\right)^{(l)}(u) d u=\int_{Y(\mathbb{A})} \int_{C X(F) \backslash C X(\mathbb{A})} \varphi^{\prime}(u y)\left(\psi^{-1}\right)^{(l)}(u) d u d y .
$$

Here $\psi^{(l)}$ is extended trivially on $X$. Put

$$
w^{\prime}=\left(\begin{array}{cccc}
I_{2 l+1} & & & \\
& & (-1)^{k-l-1} & \\
& I_{k-l-1} & & \\
& & & I_{k-l-1}
\end{array}\right) .
$$

Since $w^{\prime}$ normalizes $U^{\prime}$ and stabilizes $\left.\psi^{(l)}\right|_{U^{\prime}}$, we may replace $\varphi^{\prime}$ with its left-translate by $w^{\prime}$. The right-hand side of (4.10) becomes

$$
\int_{Y(\mathbb{A})} \int_{U^{\prime \prime}(F) \backslash U^{\prime \prime}(\mathbb{A})} \varphi\left(u w^{\prime} y\right) \psi^{-1}(u) d u d y,
$$

where

$$
\begin{aligned}
& U^{\prime \prime}=U^{\prime} \rtimes^{w^{\prime}}(C X)=\left\{\left(\begin{array}{ccccc}
z & x_{1} & x_{2} & x_{3} & x_{4} \\
& 1 & u_{1} & e & u_{2} \\
& 1 & & \\
& & & I_{k-l-1} & u_{4} \\
& & & I_{k-l-1}
\end{array}\right): z \in N_{2 l}\right\}, \\
& \psi(u)=\sum_{i=1}^{l} \psi\left((-1)^{k-i} z_{2 i-1,2 i}\right) \psi\left((-1)^{k-l-1} u_{1}\right) \psi\left(\operatorname{tr}\left(u_{4}\right)\right) .
\end{aligned}
$$

Next we show that the $d u$-integration can be extended to $U^{(l+1)}$.

Let $U^{\prime \prime \prime}<U^{\prime \prime}$ be obtained from $U^{\prime \prime}$ by zeroing out the coordinates of $u_{4}$. Define

$$
M^{\prime \prime \prime}=\left\{m(a, b)=\left(\begin{array}{ccc}
I_{2 l+1} & & \\
& 1 & a \\
& & b
\end{array}\right): b \in G_{2(k-l-1)}\right\}
$$

and denote $A^{\prime \prime \prime}=m\left(a, I_{2(k-l-1)}\right), B^{\prime \prime \prime}=m(0, b)$. The function

$$
g \mapsto \int_{U^{\prime \prime \prime}(F) \backslash U^{\prime \prime \prime}(\mathbb{A})} \varphi(u g) \psi^{-1}(u) d u
$$

is well-defined on $M^{\prime \prime \prime}(F) \backslash M^{\prime \prime \prime}(\mathbb{A})$. In particular, we can consider its Fourier expansion along the abelian unipotent subgroup $A^{\prime \prime \prime}$. There are two orbits under the action of $B^{\prime \prime \prime}(F)$, the trivial one and the one corresponding to a character $\psi^{\star}$ depending only on the leftmost coordinate of $a$. However, for each $v<\infty$, the Jaquet module $\left(\Theta_{v}\right)_{U^{\prime \prime \prime} A^{\prime \prime \prime}, \psi \psi^{\star}}=0$. To see this, first note that by Theorem 4.5 , as a vector space $\left(\Theta_{v}\right)_{U^{\prime \prime \prime} A^{\prime \prime \prime}, \psi \psi^{*}}$ is a quotient of

$$
\theta_{2 l} \otimes\left(\left(\theta_{2(k-l)}\right)_{U_{1,1,2(k-l-1)}, \psi \psi^{\star}}\right),
$$


where $U_{1,1,2(k-l-1)}$ is the unipotent radical of the standard parabolic subgroup of $G_{2(k-l)}$ with a Levi part isomorphic to $G_{1} \times G_{1} \times G_{2(k-l-1)}$ (we restricted the Jacquet module to a representation of the unipotent subgroup to pass from the metaplectic tensor to the standard one, see [Kab01, Theorem 3.1]). However, by Kap15a, Lemma 2.8] for any $m \geq 3,\left(\theta_{m}\right)_{U_{1}, \psi}$ is a quotient of $\left(\theta_{m}\right)_{U_{2}}$ (where on $U_{1}, \psi$ is given by $\left.\psi\left(\left(\begin{array}{c}{ }^{1} \\ I_{m-1}\end{array}\right)\right)=\psi\left(z_{1}\right)\right)$. Since $\psi^{\star}$ is nontrivial (and $2(k-l) \geq 4)$,

$$
\left(\theta_{2(k-l)}\right)_{U_{1,1,2(k-l-1)}, \psi \psi^{\star}}=0 .
$$

Hence only the trivial character remains and (4.12) becomes

$$
\int_{Y(\mathbb{A})} \int_{U^{(l+1)}(F) \backslash U^{(l+1)}(\mathbb{A})} \varphi\left(u w^{\prime} y\right)\left(\psi^{-1}\right)^{(l+1)}(u) d u d y .
$$

Plugging this into the left-hand side of (4.9) and using the fact that

$$
\left(w^{(l)}\right)^{-1} Y w^{(l)} V^{(l)}=V^{(l+1)}, \quad w^{\prime} w^{(l)}=w^{(l+1)}
$$

leads to the desired equality.

Theorem 4.22. The mapping $\varphi \mapsto \varphi^{U_{k}, \psi_{k}}$ on the space of $\Theta\left(=\Theta_{2 k}\right)$ is nonzero and satisfies

$$
\varphi^{U_{k}, \psi_{k}}\left(c^{\triangle}\right)=\gamma_{\psi,(-1)^{k}}(\operatorname{det} c) \varphi^{U_{k}, \psi_{k}}(1), \quad \forall c \in G_{k}(\mathbb{A}) .
$$

Consequently, $\Theta$ admits a global metaplectic Shalika model given by a Fourier coefficient. Moreover, the functional $\varphi \mapsto \varphi^{U_{k}, \psi_{k}}(1)$ is factorizable.

Proof. We begin with the proof of the formula, the non triviality will be deduced during the computation. First we show that for $c \in \mathrm{SL}_{k}(\mathbb{A})$,

$$
\varphi^{U_{k}, \psi_{k}}\left(c^{\triangle}\right)=\varphi^{U_{k}, \psi_{k}}(1)
$$

To show this, because $\varphi$ is invariant on the left by $G_{n}(F)$, it is enough to consider

$$
c=\left(\begin{array}{ccc}
1 & 0 & x \\
& I_{k-2} & 0 \\
& & 1
\end{array}\right), \quad x \in \mathbb{A} \text {. }
$$

As in the proof of Proposition 4.6 we consider the Fourier expansion along the abelian subgroup of matrices $c^{\triangle}$ with $c$ of this form. The nontrivial coefficients vanish by Theorem 4.5, This proves (4.14).

It remains to consider $c=t=\operatorname{diag}\left(t_{1}, \ldots, t_{k}\right) \in T_{k}$. To this end we claim the following.

\section{Claim 4.23.}

$$
{ }^{w} t=\prod_{l=1}^{k-1}\left(t_{l}, \prod_{i=l+1}^{k} t_{i}\right)_{2}\left(\prod_{i=l+1}^{k} t_{i}, \prod_{i=l+1}^{k} t_{i}\right)_{2} \operatorname{diag}\left(t_{1}, t_{1}, \ldots, t_{k}, t_{k}\right)
$$

\section{Claim 4.24.}

$$
\varphi^{N_{n}, \psi^{\circ}}\left(\operatorname{diag}\left(t_{1}, t_{1}, \ldots, t_{k}, t_{k}\right)\right)=\prod_{l=1}^{k} \gamma_{\psi,(-1)^{k+1-i}}\left(t_{i}\right) \delta_{B_{k}}\left(\operatorname{diag}\left(t_{1}, \ldots, t_{k}\right)\right) \varphi(1) .
$$


As a corollary, the Fourier coefficient $\varphi^{U_{k}, \psi_{k}}$ does not vanish identically on the space of $\Theta$. Indeed, the arguments in Lemma 4.19 can be repeated in "the opposite direction" to relate $\varphi^{N_{n}, \psi^{\circ}}$ to an integration of $\varphi^{U_{k}, \psi_{k}}$. The former is nonzero by Claim 4.24, hence $\varphi^{U_{k}, \psi_{k}}$ cannot be zero for all $\varphi$.

According to (1.17), for any $1 \leq l \leq k-2$,

$$
\begin{aligned}
& \left(t_{l}, \prod_{i=l+1}^{k} t_{i}\right)_{2}\left(\prod_{i=l+1}^{k} t_{i}, \prod_{i=l+1}^{k} t_{i}\right)_{2} \gamma_{\psi,(-1)^{k+1-l}}\left(t_{l}\right) \gamma_{\psi,(-1)^{k+1-l+1}}\left(\prod_{i=l+1}^{k} t_{i}\right) \\
& =\left(t_{l}, \prod_{i=l+1}^{k} t_{i}\right)_{2} \gamma_{\psi,(-1)^{k+1-l}}\left(t_{l}\right) \gamma_{\psi,(-1)^{k+1-l}}\left(\prod_{i=l+1}^{k} t_{i}\right)=\gamma_{\psi,(-1)^{k+1-l}}\left(\prod_{i=l}^{k} t_{i}\right) .
\end{aligned}
$$

The conjugation of $V$ by $t$ multiplies the measure by $\delta_{B_{k}}^{-1}\left(\operatorname{diag}\left(t_{1}, \ldots, t_{k}\right)\right)$. Combining the claims yields the result.

The assertion regarding factorizability follows from the local uniqueness of the metaplectic Shalika model at all the local components of $\Theta$ (see $\S 4.2$, we only need to know that the dimension of the space of metaplectic Shalika functionals on $\Theta_{v}$ is at most one, for all $v$ ).

Proof of Claim 4.23. This follows from the local properties of the cocycle of [BLS99]. Specifically, write $t$ in the form $\operatorname{diag}\left(t_{1}, t^{\prime}, t_{1}, t^{\prime}\right) \in T_{n}$ with $t^{\prime} \in T_{k-1}$ and let $w^{\prime}$ be given by (4.11) with $l=0$. Using [BLS99] (§ 2 Lemma 2, $\S 3$ Lemmas 3 and 1) we see that

$$
\begin{aligned}
w^{w^{\prime}} \mathfrak{s}(t) & =\sigma\left(w^{\prime}, t\right) \mathfrak{s}\left({ }^{w} t\right)=\left(t_{1}, \operatorname{det} t^{\prime}\right)_{2}\left(\operatorname{det} t^{\prime}, \operatorname{det} t^{\prime}\right)_{2} \mathfrak{s}\left(\operatorname{diag}\left(t_{1}, t_{1}, t^{\prime}, t^{\prime}\right)\right) \\
& =\left(t_{1}, \operatorname{det} t^{\prime}\right)_{2}\left(\operatorname{det} t^{\prime}, \operatorname{det} t^{\prime}\right)_{2} \mathfrak{s}\left(\operatorname{diag}\left(t_{1}, t_{1}, I_{2 n-2}\right)\right) \mathfrak{s}\left(\operatorname{diag}\left(I_{2}, t^{\prime}, t^{\prime}\right)\right) .
\end{aligned}
$$

For $l=1, w^{\prime}$ commutes with $\mathfrak{s}\left(\operatorname{diag}\left(t_{1}, t_{1}, I_{2 n-2}\right)\right)$, and by (1.1) we can apply the same arguments to compute ${ }^{w^{\prime}} \mathfrak{s}\left(\operatorname{diag}\left(I_{2}, t^{\prime}, t^{\prime}\right)\right)$. Since $w$ is a product if elements $w^{\prime}$ (with $l$ varying), we can repeat this for all $l=1, \ldots, k-1$ and obtain the result.

Proof of Claim 4.24. Let $Q=M \ltimes U<G_{n}$ be the standard parabolic subgroup, whose Levi part $M$ is isomorphic to $k$ copies of $G_{2}$. According to Theorem 4.8, the mapping $m \mapsto \varphi^{U}(m)$ belongs to the space of $\delta_{Q}^{1 / 4} \Theta_{M}$. The representation $\Theta_{M}$ is isomorphic to the global Weil representation $\Pi \otimes \widetilde{\varphi}^{-1}$ constructed by Takeda [Tak14, $\S 2.3$ and p. 204] (this is $\Pi_{\chi} \otimes \widetilde{\varphi}_{P}^{-1}$ in his notation with the unitary character $\chi=1$, see [Tak14, 2.26]). The mapping $\widetilde{\varphi}$ was defined in [Tak14, $\S$ A.1, p. 261] to correct a global block-compatibility issue. If $m \in M(\mathbb{A})$, $\widetilde{\varphi}(m)=m \widehat{S}(m)$, where $\widehat{S}=\prod_{v}(\widehat{S})_{v}\left(m_{v}\right)$ and at each place $v$,

$$
(\widehat{S})_{v}(m)=\frac{\prod_{i=1}^{k} \mathfrak{s}_{v}\left(m_{i}\right)}{\mathfrak{s}_{v}(m)}, \quad m=\operatorname{diag}\left(m_{1}, \ldots, m_{k}\right) \in M\left(F_{v}\right) .
$$

Therefore $\varphi^{N_{n}, \psi^{\circ}}$ is the application of a Fourier coefficient corresponding to $\left(N_{M},\left.\psi^{\circ}\right|_{N_{M}}\right)$, to an automorphic form in the space of $\delta_{Q}^{1 / 4}\left(\Pi \otimes \widetilde{\varphi}^{-1}\right)$.

For $d=\operatorname{diag}\left(t_{1}, t_{1}, \ldots, t_{k}, t_{k}\right), \widehat{S}(d)=1$ because for all $v, \prod_{i=1}^{k} \mathfrak{s}_{v}\left(t_{i}^{\triangle}\right)=\mathfrak{s}_{v}(t)$. Hence $\widetilde{\varphi}(d)=d$. Also $\delta_{Q}^{1 / 4}(d)=\delta_{B_{k}}\left(\operatorname{diag}\left(t_{1}, \ldots, t_{k}\right)\right)$. Lastly, following the definitions and formulas from [Tak14, Proposition 2.55 and (2.56)] we see that for any automorphic form $\phi$ in the space of $\Pi, \phi^{N_{M}, \psi^{\circ}}(d)=\prod_{l=1}^{k} \gamma_{\psi,(-1)^{k+1-i}}\left(t_{i}\right) \phi(1)$.

Corollary 4.25. Let $\theta\left(=\theta_{2 k}\right)$ be an exceptional representation over an Archimedean field (in fact, over any local field). Then $\theta$ admits a metaplectic $\left(\psi_{(-1)^{k}}, \psi\right)$-Shalika functional. 
Proof. The functional $\varphi \mapsto \varphi^{U_{k}, \psi_{k}}(1)$ defines a metaplectic $\left(\psi_{(-1)^{k}}, \psi\right)$-Shalika functional on each of the local pieces of $\Theta$. Now the result follows from Theorem 4.22 and a standard globalization argument applied to the exceptional representation.

\section{A Godement-JACQUet type Integral}

5.1. Local theory. Let $F$ be a local field and $\pi \in \operatorname{Alg} G_{k}$ be irreducible. Set $n=2 k$ and $\theta=\theta_{n}\left(\theta_{n}\right.$ was defined in $\S$ 4.1). Regard $G_{k}$ as a subgroup of $G_{n}$ via the embedding $g \mapsto \operatorname{diag}\left(g, I_{k}\right)$.

We define the following local Godement-Jacquet type integral. Let $f$ be a matrix coefficient of $\pi, \mathscr{S} \in \mathscr{S}\left(\theta, \psi_{(-1)^{k}}, \psi\right), \mathscr{S}^{\prime} \in \mathscr{S}\left(\theta, \psi_{(-1)^{k+1}}, \psi^{-1}\right)$ and $s \in \mathbb{C}$. Define

$$
Z\left(f, \mathscr{S}, \mathscr{S}^{\prime}, s\right)=\int_{G_{k}} f(g) \mathscr{S}(\mathfrak{s}(g)) \mathscr{S}^{\prime}(\mathfrak{s}(g))|\operatorname{det} g|^{s-k / 2} d g .
$$

Note that the actual choice of section $\mathfrak{s}$ here does not matter, because $\mathscr{S}$ and $\mathscr{S}^{\prime}$ are both genuine. Henceforth we omit it from the notation.

Theorem 5.1. Assume $F$ is a p-adic field.

(1) The integral (5.1) is absolutely convergent for $\operatorname{Re}(s) \gg 0$, the domain of convergence depends only on the representations.

(2) One can choose data $\left(f, \mathscr{S}, \mathscr{S}^{\prime}\right)$ such that $Z\left(f, \mathscr{S}, \mathscr{S}^{\prime}, s\right)$ is absolutely convergent and equals 1 , for all $s$.

(3) The integral has a meromorphic continuation to a rational function in $\mathbb{C}\left(q^{-s}\right)$.

(4) When all data are unramified,

$$
Z\left(f, \mathscr{S}, \mathscr{S}^{\prime}, s\right)=\frac{L\left(2 s, \mathrm{Sym}^{2}, \pi\right)}{L\left(2 s+1, \wedge^{2}, \pi\right)} .
$$

(5) Assume that $\pi$ satisfies the following condition: if $\varepsilon$ is a normalized exponent of $\pi$ along a standard parabolic subgroup $Q$ (see $\S 1.4$ for the definition), then $\delta_{Q}^{1 / 2}|\varepsilon|$ lies in the open cone spanned by the positive roots in the Levi part of $Q$. In particular, this condition holds when $\pi$ is tempered. Then $Z\left(f, \mathscr{S}, \mathscr{S}^{\prime}, s\right)$ is holomorphic at $\operatorname{Re}(s)=1 / 2$.

Proof. The first three assertions have already been proved in Kap15a, in a slightly different form. By virtue of Claim 3.2 the integral is bounded by the zeta integral of Godement and Jacquet,

$$
\int_{G} f(g) \phi(g)|\operatorname{det}|^{r}(g) d g
$$

where $\phi \in \mathcal{S}\left(F_{k \times k}\right)$ is positive and $r \in \mathbb{R}$ depends only on $\theta$ (i.e., not on $\mathscr{S}$ and $\mathscr{S}^{\prime}$ ). This integral is absolutely convergent for $r>r_{0}$, where $r_{0}$ is independent of the specific coefficient $f$ ([GJ72, p. 30]).

Applying Claim 3.3 to $\theta$, one can choose elements $\mathscr{S}$ and $\mathscr{S}^{\prime}$ such that the mapping $g \mapsto \mathscr{S}(g) \mathscr{S}^{\prime}(g)$ is an arbitrary function in $\operatorname{ind}_{G_{k}^{\Delta} U_{k}}^{Q_{n}}(1) \cong C_{c}^{\infty}\left(G_{k}\right)$. This implies (2), when we take some $f$ which does not vanish at the identity.

We turn to the meromorphic continuation. For $a, b \in G_{k}$, put $m=\operatorname{diag}(a, b) \in M_{k}$. Then for any $g \in G_{k}$,

$$
\mathscr{S}(g m) \mathscr{S}^{\prime}(g m)=\left(\mathscr{S} \mathscr{S}^{\prime}\right)\left(\operatorname{diag}\left(g, I_{k}\right) m\right)
$$




$$
\begin{aligned}
& =\gamma_{\psi}(\operatorname{det} b) \gamma_{\psi}^{-1}(\operatorname{det} b)\left(\mathscr{S} \mathscr{S}^{\prime}\right)\left(\operatorname{diag}\left(b^{-1} g a, I_{k}\right)\right) \\
& =\left(\mathscr{S} \mathscr{S}^{\prime}\right)\left(b^{-1} g a\right) .
\end{aligned}
$$

Additionally for $u \in U_{k}$,

$$
\mathscr{S}(g u) \mathscr{S}^{\prime}(g u)=\psi(g u) \psi^{-1}(g u)\left(\mathscr{S} \mathscr{S}^{\prime}\right)(g)=\left(\mathscr{S} \mathscr{S}^{\prime}\right)(g) .
$$

Hence in its domain of absolute convergence (5.1) satisfies

$$
Z\left(m f, m u \mathscr{S}, m u \mathscr{S}^{\prime}, s\right)=\delta_{Q_{k}}^{1 / 2-s / k}(m) Z\left(f, \mathscr{S}, \mathscr{S}^{\prime}, s\right) .
$$

Therefore it is a nonzero element of

$$
\operatorname{Hom}_{M_{k}}\left(\left(\theta \otimes \theta^{\prime}\right)_{U_{k}}, \delta_{Q_{k}}^{1 / 2}|\operatorname{det}|^{-s} \pi^{\vee} \otimes|\operatorname{det}|^{s} \pi\right) .
$$

In [Kap15a, Claim 4.6] we proved that this space is at most one-dimensional, outside of a finite set of values of $q^{-s}$. We mention that the proof utilized the structure of the twisted Jacquet modules of $\theta$ along $U_{k}$.

Now the one-dimensionality result combined with the first two assertions imply (3), by virtue of Bernstein's continuation principle (in [Ban98]).

Assume all data are unramified. We prove (4). The function $f$ is bi- $G_{k}(\mathcal{O})$-invariant and by Claim 3.27 (1) , for any $k_{1}, k_{2} \in G_{k}(\mathcal{O})$,

$$
\left(\mathscr{S} \mathscr{S}^{\prime}\right)\left(k_{1} g k_{2}\right)=\left(\mathscr{S} \mathscr{S}^{\prime}\right)(g) .
$$

Note that on its own, $\mathscr{S}$ is not bi- $\kappa\left(G_{k}(\mathcal{O})\right)$-invariant because elements of $\kappa\left(\operatorname{diag}\left(I_{k}, G_{k}(\mathcal{O})\right)\right)$ and $\mathfrak{s}\left(\operatorname{diag}\left(G_{k}, I_{k}\right)\right)$ do not commute in $\widetilde{G}_{n}$. Nonetheless, the integrand is bi- $G_{k}(\mathcal{O})$-invariant. Hence we can write (5.1) using the Cartan decomposition $\coprod_{\lambda \in \mathbb{Z}_{\geq}^{k}} G_{k}(\mathcal{O}) \varpi^{\lambda} G_{k}(\mathcal{O})$ and Lemma 4.2 implies that the integrand vanishes unless $\lambda \in 2 \mathbb{Z}_{+}^{k}$ (see $\S 3.3$ for the notation).

Let $t_{\pi} \in G_{k}(\mathbb{C})$ be the Satake parameters of $\pi$. For $\lambda \in \mathbb{Z}_{+}^{k}$, the formulas of Macdonald [Mac95, Chapter V $\S 2(2.9)$ and $\S 3(3.4)]\left(f\left(\varpi^{\lambda}\right)\right.$ is $\omega_{s}\left(\pi^{-\lambda}\right)$ in his notation) show

$$
f\left(\varpi^{\lambda}\right)=\operatorname{vol}\left(G_{k}(\mathcal{O}) \varpi^{\lambda} G_{k}(\mathcal{O})\right)^{-1} \delta_{B_{k}}^{-1 / 2}\left(\varpi^{\lambda}\right) P_{\lambda}\left(t_{\pi} ; q^{-1}\right) .
$$

Here $P_{\lambda}$ is the Hall-Littlewood polynomial in the Satake parameters, see [Mac95, Chapter III $\S 2]$ for the definition. Using Lemma 4.2 and $\delta_{B_{n}}^{1 / 2}\left(t_{\lambda}\right)\left|\operatorname{det} \varpi^{\lambda}\right|^{-k / 2}=\delta_{B_{k}}^{1 / 2}\left(\varpi^{\lambda}\right)$, the integral becomes

$$
\sum_{\lambda \in 2 \mathbb{Z}_{+}^{k}} P_{\lambda}\left(t_{\pi} ; q^{-1}\right) q^{-|\lambda| s}, \quad|\lambda|=\lambda_{1}+\ldots+\lambda_{k} .
$$

Since $P_{\lambda}\left(t_{\pi} ; q^{-1}\right) q^{-|\lambda| s}=P_{\lambda}\left(t_{\pi|\operatorname{det}| s} ; q^{-1}\right)$, the required formula follows immediately from the formal identity of [Mac95, Chapter III $\S 5$, example 2]:

$$
\sum_{\lambda \in 2 \mathbb{Z}_{+}^{k}} P_{\lambda}\left(x_{1}, \ldots, x_{k} ; r\right)=\frac{\prod_{i<j}\left(1-r x_{i} x_{j}\right)}{\prod_{i \leq j}\left(1-x_{i} x_{j}\right)} .
$$

Finally consider (5). Assume $\pi$ satisfies the assumption on the exponents. We prove that the integral is absolutely convergent, uniformly on compact sets, if $r=\operatorname{Re}(s) \geq 1 / 2-\epsilon_{0}$, for some $\epsilon_{0}>0$ (depending on the exponents of $\pi$ ). Let $\Delta \subset \Delta_{G_{k}}$ and $P=P_{\Delta}$ be the corresponding standard parabolic subgroup, $P=M \ltimes U$. Let $\zeta>0$. Let $C_{M}^{+}$be the set of $\varpi^{\lambda} \in C_{M}$ such that $|\alpha(t)|<\zeta$ for all $\alpha \in \Delta_{G_{k}}-\Delta$. Let $\mathcal{K}$ be a principal congruence subgroup on which the integrand is bi- $\mathcal{K}$. For the proof of convergence, we may replace $K$ 
in the Cartan decomposition with $\mathcal{K}$, and we see that it is enough to prove the convergence of the following sum, for each $\Delta$,

$$
\sum_{\varpi^{\lambda} \in C_{M}^{+}}\left|f \mathscr{S} \mathscr{S}^{\prime} \delta_{B_{k}}^{-1}\right|\left(\varpi^{\lambda}\right) q^{-|\lambda|(r-k / 2)} .
$$

Here the modulus character $\delta_{B_{k}}^{-1}$ is bounding $\operatorname{vol}\left(\mathcal{K} \varpi^{\lambda} \mathcal{K}\right)$.

This sum can be bounded using the exponents of the representations. Let $P^{\wedge}$ be the standard parabolic subgroup of $G_{n}$, whose Levi part is isomorphic to $M \times G_{k}$. According to [JR96, Lemma 6.2], if $\zeta$ is sufficiently small with respect to $\varphi, \mathscr{S}$ is bounded on $C_{M}^{+}$by the exponents of $\theta$ along $P^{\wedge}$. Kable proved that the normalized Jacquet module of $\theta$ along the unipotent radical of $P^{\wedge}$ is an irreducible representation with a central character $\omega$ such that $|\omega|=\delta_{P^{\wedge}}^{-1 / 4}$ [Kab01, Theorem 5.1]. The normalized exponent of $\theta$ along $P^{\wedge}$ is thus $\delta_{P^{\wedge}}^{-1 / 4}$. Since on $C_{M}$, $|\operatorname{det}|^{-k / 4} \delta_{B_{k}}^{-1 / 4}=\delta_{P^{\wedge}}^{-1 / 4}$ (on the right-hand side $C_{M}$ is embedded in $M$ ), we obtain (up to a constant)

$$
\sum_{\varpi^{\lambda} \in C_{M}^{+}}\left|\delta_{B_{k}}^{-1 / 2} f\right|\left(\varpi^{\lambda}\right) q^{-|\lambda| r} .
$$

This is bounded in the proper right half-plane, given our assumption on $\pi$.

Remark 5.2. As we have seen in the proof, the local zeta integral can be regarded as an element of (5.2), which according to Kap15a, Claim 4.6] is at most one-dimensional, outside of a finite set of values of $q^{-s}$. This is the uniqueness property underlying the standard definition of the local gamma factor of Rankin-Selberg integrals. One typically considers a "similar zeta integral", absolutely convergent in a left half-plane, which also belongs to (5.2), then the proportionality factor between these integrals is the gamma factor. The problem is to find the similar integral. It should be related to a global construction, because the product of local gamma factors, over all places (including the Archimedean ones) should be 1, and this usually follows from a global functional equation.

Theorem 5.3. Assume $F$ is an Archimedean field.

(1) The integral (5.1) is absolutely convergent for $\operatorname{Re}(s) \gg 0$, the domain of convergence depends only on the representations.

(2) For each $r>0$ one can choose data $\left(f, \mathscr{S}, \mathscr{S}^{\prime}\right)$ such that $Z\left(f, \mathscr{S}, \mathscr{S}^{\prime}, s\right)$ is absolutely convergent and nonzero for $s$ in the strip $\{s \in \mathbb{C}:|\operatorname{Im}(s)| \leq r\}$.

(3) For fixed $\left(f, \mathscr{S}, \mathscr{S}^{\prime}\right)$ the function $Z\left(f, \mathscr{S}, \mathscr{S}^{\prime}, s\right)$ has a meromorphic continuation in $s \in \mathbb{C}$.

Proof. As in the $p$-adic case, by Claim 3.2 the convergence is reduced to the convergence of a Godement-Jacquet type integral, which was proved in GJ72, Theorem 8.7].

Consider (2). Let $f$ be a matrix coefficient with $f\left(I_{k}\right)=1$. We choose a compact neighborhood $\mathcal{V}$ of the identity in $G_{k}$, such that both $f$ and $|\operatorname{det}|^{s-k / 2}$ take values in $\{z \in \mathbb{C}: \arg (z)<\pi / 8\}$, on $\mathcal{V}$ and whenever $|\operatorname{Im}(s)| \leq r$. We will show that, possibly taking a smaller $\mathcal{V}$, one can choose $\mathscr{S}$ and $\mathscr{S}^{\prime}$ such that $\mathscr{S} \mathscr{S}^{\prime}(g)$ is supported in $\mathcal{V}$, is equal to 1 at the identity, and takes values in $\{\arg (z)<\pi / 4\}$. We see that the integrand is supported in $\mathcal{V}$, equals 1 at the identity, and in its support takes values in $\{\operatorname{Re}(z)>0\}$, for $|\operatorname{Im}(s)| \leq r$. Hence, the corresponding integral over $G_{k}$ converges for any such $s$ and is nonzero. 
To find $\mathscr{S}$ and $\mathscr{S}^{\prime}$ with the above properties, we imitate the argument used in the proof of Claim 3.3. In fact, this argument shows that for any $\varphi$ in the representation space of $\theta$ and any $\phi \in \mathcal{S}\left(F_{k \times k}\right), \mathscr{S}_{\theta(\phi) \varphi}\left(d_{g}\right)=\widehat{\phi}(g) \mathscr{S}_{\varphi}\left(d_{g}\right)$, where $d_{g}=\mathfrak{s}\left(\operatorname{diag}\left(g, I_{k}\right)\right)$. We choose $\varphi$ such that $\mathscr{S}_{\varphi}\left(\mathfrak{s}\left(I_{n}\right)\right)=1$, then possibly making $\mathcal{V}$ smaller we can achieve that $\mathscr{S}_{\varphi}\left(d_{g}\right)$ takes values in $\{\arg (z)<\pi / 8\}$ for $g \in \mathcal{V}$. We take $\phi$ such that $\widehat{\phi} \geq 0, \phi\left(I_{k}\right)=1$, and has support in $\mathcal{V}$, then put $\mathscr{S}=\mathscr{S}_{\theta(\phi) \varphi}$. The function $\mathscr{S}^{\prime}$ is chosen similarly.

Now we prove (3). As explained in the proof of Claim 3.2 , we may replace $\mathscr{S}$ by a finite $\operatorname{sum} \sum_{j} \mathscr{S}_{j} \phi_{j}$ with metaplectic Shalika functions $\mathscr{S}_{j}$ and $\phi_{j} \in \mathcal{S}\left(F_{k \times k}\right)$. Hence it is enough to prove meromorphic continuation for an integral of the form

$$
\int_{G_{k}} f(g) \mathscr{S} \mathscr{S}^{\prime}(g) \phi(g)|\operatorname{det}(g)|^{s-k / 2} d g, \quad \phi \in \mathcal{S}\left(F_{k \times k}\right) .
$$

We use the integration formula

$$
\int_{G_{k}} \vartheta(g) d g=\int_{K} \int_{K} \int_{\mathbb{R}} \int_{\mathbb{R}_{>0}^{k-1}} \vartheta\left(k_{1} a_{x} k_{2}\right) J(x) d\left(x_{1}, \ldots, x_{k-1}\right) d x_{k} d k_{1} d k_{2},
$$

where $K$ is the maximal compact subgroup of $G_{k}$ (the orthogonal group over $\mathbb{R}$, the unitary group if $F=\mathbb{C}$ ) and for $x=\left(x_{1}, \ldots, x_{k}\right) \in \mathbb{R}^{k}$,

$$
\begin{aligned}
& a_{x}=\operatorname{diag}\left(e^{-\left(x_{1}+\cdots+x_{k}\right)}, e^{-\left(x_{2}+\cdots+x_{k}\right)}, \ldots, e^{-x_{k}}\right), \\
& J(x)=\prod_{1 \leq i<j \leq k} \sinh \left(x_{i}+\cdots+x_{j-1}\right),
\end{aligned}
$$

for $F=\mathbb{R}$, and $J(x)$ has to be replaced by $J(x)^{2}$ if $F=\mathbb{C}$. Since $J(x)$ is a finite sum of terms of the form $e^{p \cdot x^{\prime}}$ with $p \in \mathbb{Z}^{k-1}$ and $x^{\prime}=\left(x_{1}, \ldots, x_{k-1}\right)$, it suffices to show that every integral of the form

$$
\int_{K} \int_{K} \int_{\mathbb{R}} \int_{\mathbb{R}_{>0}^{k-1}} f \mathscr{S} \mathscr{S}^{\prime} \phi\left(k_{1} a_{x} k_{2}\right) e^{-\left(s+s_{1}\right) x_{1}} e^{-\left(2 s+s_{2}\right) x_{2}} \cdots e^{-\left(k s+s_{k}\right) x_{k}} d(\cdots)
$$

with $s_{1}, \ldots, s_{k} \in \mathbb{R}$ (coming from the exponent $k / 2$ in $|\operatorname{det}(g)|^{s-k / 2}$ and from $e^{p \cdot x^{\prime}}$ ) converges absolutely for $\operatorname{Re}(s) \gg 0$ and extends to a meromorphic function in $s \in \mathbb{C}$.

Note that $f(g)=\xi^{\vee}(\pi(g) \xi)$ for some $\xi \in V$ and $\xi^{\vee} \in V^{\vee}$, where $V$ is the space of $\pi$, so that

$$
f\left(k_{1} a_{x} k_{2}\right)=\pi^{\vee}\left(k_{1}^{-1}\right) \xi^{\vee}\left(\pi\left(a_{x}\right) \pi\left(k_{2}\right) \xi\right) .
$$

Also $k_{1} \mapsto \pi^{\vee}\left(k_{1}^{-1}\right) \xi^{\vee}$ and $k_{2} \mapsto \pi\left(k_{2}\right) \xi$ are smooth. Hence Theorem 1.1 can be applied with $\pi^{\vee}\left(k_{1}^{-1}\right) \xi^{\vee}$ instead of $\xi^{\vee}$ and $\pi\left(k_{2}\right) \xi$ instead of $\xi$. Furthermore $\mathscr{S}$ (resp. $\mathscr{S}^{\prime}$ ) is of the form $\mathscr{S}(g)=l(\theta(g) \varphi)\left(\right.$ resp. $\left.\mathscr{S}^{\prime}(g)=l^{\prime}\left(\theta(g) \varphi^{\prime}\right)\right)$ with $\varphi$ (resp. $\left.\varphi^{\prime}\right)$ in the space of $\theta$ and a metaplectic Shalika functional $l$ (resp. $l^{\prime}$ ). Then (3.2) implies

$$
\mathscr{S} \mathscr{S}^{\prime}\left(k_{1} a_{x} k_{2}\right)=\mathscr{S} \mathscr{S}^{\prime}\left(a_{x} \operatorname{diag}\left(k_{2}, k_{1}^{-1}\right)\right),
$$

and we can apply Theorem 3.5 with $\theta\left(\mathfrak{s}\left(\operatorname{diag}\left(k_{2}, k_{1}^{-1}\right)\right)\right) \varphi$ instead of $\varphi\left(\right.$ similarly for $\left.\varphi^{\prime}\right)$.

We split the $d x_{k}$-integration in (5.3) into $x_{k} \geq 0$ and $x_{k} \leq 0$. First assume $x_{k} \geq 0$. By the above considerations and Theorems 1.1 and 3.5, $f \mathscr{S} \mathscr{S}^{\prime}\left(k_{1} a_{x} k_{2}\right)$ can be written as a finite sum of terms of the form

$$
c\left(x_{\bar{I}} ; k_{1}, k_{2}\right) x_{I}^{\alpha} e^{-z \cdot x_{I}}
$$


where $I \subset\{1, \ldots, k\}, \alpha \in \mathbb{N}^{I}, z \in \mathbb{C}^{I}$ with $\operatorname{Re} z_{j} \geq \Lambda_{j}=\Lambda_{\pi, j}+\Lambda_{\theta, j}+\Lambda_{\theta^{\prime}, j}$ for all $j \in I$ (see (1.3) for the definition of $\left.\Lambda_{\pi, j}\right)$, and $c\left(x_{\bar{I}} ; k_{1}, k_{2}\right)$ is smooth in $x_{\bar{I}}, k_{1}$ and $k_{2}$, and satisfies

$$
\left|c\left(x_{\bar{I}} ; k_{1}, k_{2}\right)\right| \leq C e^{-D_{\bar{I}} \cdot x_{\bar{I}}}, \quad \forall x_{\bar{I}} \in \mathbb{R}_{>0}^{\bar{I}}, k_{1}, k_{2} \in K .
$$

Here the numbers $D_{j} \in \mathbb{R}$ can be chosen arbitrarily large. The resulting integral is

$$
\int_{K} \int_{K} \int_{\mathbb{R}_{>0}^{k}} c\left(x_{\bar{I}} ; k_{1}, k_{2}\right) x_{I}^{\alpha} e^{-z \cdot x_{I}} \phi\left(k_{1} a_{x} k_{2}\right)\left(\prod_{j=1}^{k} e^{-\left(j s+s_{j}\right) x_{j}}\right) d(\cdots) .
$$

Substitute $y_{j}=e^{-x_{j}}$ and obtain

$$
\int_{K} \int_{K} \int_{0}^{1} \cdots \int_{0}^{1} c^{\prime}\left(y_{\bar{I}} ; k_{1}, k_{2}\right)\left(\prod_{j \in I} y_{j}^{j s+s_{j}+z_{j}} \log ^{\alpha_{j}} y_{j}\right)\left(\prod_{j \notin I} y_{j}^{j s+s_{j}}\right) \phi\left(k_{1} b_{y} k_{2}\right) d(\cdots),
$$

where $b_{y}=\operatorname{diag}\left(y_{1} \cdots y_{k}, y_{2} \cdots y_{k}, \ldots, y_{k}\right)$ and $c^{\prime}\left(e^{-x_{\bar{I}}} ; k_{1}, k_{2}\right)=c\left(x_{\bar{I}} ; k_{1}, k_{2}\right)$ satisfies

$$
\left|c^{\prime}\left(y_{\bar{I}} ; k_{1}, k_{2}\right)\right| \leq C y_{\bar{I}}^{D_{\bar{I}}}, \quad \forall y_{\bar{I}} \in(0,1)^{\bar{I}}, k_{1}, k_{2} \in K .
$$

The function $\phi\left(k_{1} b_{y} k_{2}\right)$ together with all its $y$-derivatives is bounded for all $k_{1}, k_{2} \in K$ and $y_{1}, \ldots, y_{k} \in(0,1)$. Hence, the integral

$$
B_{c^{\prime}}(y)=\int_{K} \int_{K} c^{\prime}\left(y_{\bar{I}} ; k_{1}, k_{2}\right) \phi\left(k_{1} b_{y} k_{2}\right) d k_{1} d k_{2}
$$

converges absolutely and defines a smooth function in $y_{1}, \ldots, y_{k} \in(0,1)$ such that for all differential operators $\mathcal{D}$ in $y_{I}$ with constant coefficients we have

$$
\left|\mathcal{D} B_{c^{\prime}}(y)\right| \leq C_{\mathcal{D}} y_{\bar{I}}^{M_{\bar{I}}}, \quad \forall y \in(0,1)^{k} .
$$

This implies that the integral

$$
\int_{0}^{1} \cdots \int_{0}^{1} B_{c^{\prime}}(y)\left(\prod_{j \in I} y_{j}^{j s+s_{j}+z_{j}} \log ^{\alpha_{j}} y_{j}\right)\left(\prod_{j \notin I} y_{j}^{j s+s_{j}}\right) d y_{1} \cdots d y_{k}
$$

converges absolutely whenever $\operatorname{Re} s>-\frac{\operatorname{Re} \Lambda_{j}+s_{j}+1}{j} \geq-\frac{\operatorname{Re} z_{j}+s_{j}+1}{j}$ for $j \in I$ and $\operatorname{Re} s>$ $-\frac{D_{j}+s_{j}+1}{j}$ for $j \notin I$. By the usual technique writing $(s+1) y^{s}=\frac{d}{d y} y^{s+1}$ and integrating by parts it is easy to see that the integral further extends to a meromorphic function in the region where $\operatorname{Re} s>-\frac{D_{j}+s_{j}+1}{j}$ for $j \notin I$ (the region where the integrals over $y_{\bar{I}}$ always converge absolutely). Since the $D_{j}$ can be chosen arbitrarily large, this shows the meromorphic extension to $s \in \mathbb{C}$.

Now assume $x_{k} \leq 0$. Let $x^{\prime}=\left(x_{1}, \ldots, x_{k-1}, 0\right)$ with $x_{1}, \ldots, x_{k-1} \geq 0$ and $x_{k} \leq 0$. By Theorems 1.1 and 3.5 and Remark 3.6 the product $f \mathscr{S} \mathscr{S}^{\prime}\left(k_{1} a_{x} k_{2}\right)$ can be written as a finite sum of terms of the form

$$
c\left(x_{\bar{J}}^{\prime}, x_{k} ; k_{1}, k_{2}\right)\left(x_{J}^{\prime}\right)^{\alpha} e^{-z^{\prime} \cdot x_{J}^{\prime}}
$$

where $J \subset\{1, \ldots, k-1\}, \bar{J}=\{1, \ldots, k-1\}-J, \alpha \in \mathbb{N}^{J}, z^{\prime} \in \mathbb{C}^{J}$ with $\operatorname{Re} z_{j}^{\prime} \geq \Lambda_{j}=$ $\Lambda_{\pi, j}+\Lambda_{\theta, j}+\Lambda_{\theta^{\prime}, j}$ for all $j \in J$, and $c\left(x_{\bar{J}}^{\prime}, x_{k} ; k_{1}, k_{2}\right)$ is smooth in $x_{\bar{J}}^{\prime}, x_{k}, k_{1}$ and $k_{2}$, and satisfies

$$
\left|c\left(x_{\bar{J}}^{\prime}, x_{k} ; k_{1}, k_{2}\right)\right| \leq C e^{-D_{\bar{J}}^{\prime} \cdot x_{\bar{J}}^{\prime}} e^{-N x_{k}}, \quad \forall x_{\bar{I}} \in \mathbb{R}_{>0}^{\bar{I}}, x_{k} \leq 0, k_{1}, k_{2} \in K
$$

Here the numbers $D_{j}^{\prime} \in \mathbb{R}$ can be chosen arbitrarily large and $N \in \mathbb{R}$ depends on $D_{j}^{\prime}$. The resulting integral is 


$$
\int_{K} \int_{K} \int_{-\infty}^{0} \int_{\mathbb{R}_{>0}^{k-1}} c\left(x_{\bar{J}}^{\prime}, x_{k} ; k_{1}, k_{2}\right)\left(x_{J}^{\prime}\right)^{\alpha} e^{-z^{\prime} \cdot x_{J}^{\prime}} \phi\left(k_{1} a_{x} k_{2}\right)\left(\prod_{j=1}^{k} e^{-\left(j s+s_{j}\right) x_{j}}\right) d(\cdots) .
$$

Again substitute $y_{j}=e^{-x_{j}}$ and obtain

$$
\begin{aligned}
& \int_{K} \int_{K} \int_{1}^{\infty} \int_{0}^{1} \cdots \int_{0}^{1} c^{\prime}\left(y_{\bar{J}}^{\prime}, y_{k} ; k_{1}, k_{2}\right) \\
& \times\left(\prod_{j \in J} y_{j}^{j s+s_{j}+z_{j}} \log ^{\alpha_{j}} y_{j}\right)\left(\prod_{j \notin J} y_{j}^{j s+s_{j}}\right) \phi\left(k_{1} b_{y} k_{2}\right) d(\cdots),
\end{aligned}
$$

where $b_{y}=\operatorname{diag}\left(y_{1} \cdots y_{k}, y_{2} \cdots y_{k}, \ldots, y_{k}\right)$ and $c^{\prime}\left(e^{-x_{\frac{J}{J}}^{\prime}}, e^{-x_{k}} ; k_{1}, k_{2}\right)=c\left(x_{\frac{J}{J}}^{\prime}, x_{k} ; k_{1}, k_{2}\right)$ satisfies

$$
\left|c^{\prime}\left(y_{\bar{J}}^{\prime}, y_{k} ; k_{1}, k_{2}\right)\right| \leq C\left(y_{\bar{J}}^{\prime}\right)^{D \frac{J}{J}} y_{k}^{N}, \quad \forall y_{\bar{J}}^{\prime} \in(0,1)^{\bar{J}}, y_{k} \in(1, \infty), k_{1}, k_{2} \in K .
$$

Note that $y_{k}$ is a single coordinate in $b_{y}$ and $\phi \in \mathcal{S}\left(F_{k \times k}\right)$. This implies that the function $\phi\left(k_{1} b_{y} k_{2}\right)$ together with all its $y^{\prime}$-derivatives is bounded by a constant times $y_{k}^{-L}$ with $L \in \mathbb{R}$ arbitrarily large. Then $B_{c^{\prime}}(y)$ (defined as above) converges absolutely and defines a smooth function in $y_{1}, \ldots, y_{k-1} \in(0,1), y_{k} \in(1, \infty)$ such that for all differential operators $\mathcal{D}$ in $y_{J}^{\prime}$ with constant coefficients we have

$$
\left|\mathcal{D} B_{c^{\prime}}(y)\right| \leq C_{\mathcal{D}}\left(y_{\bar{J}}^{\prime}\right)^{D_{\bar{J}}^{\prime}} y_{k}^{N-L}, \quad \forall y^{\prime} \in(0,1)^{k-1}, y_{k} \in(1, \infty) .
$$

This implies that the integral

$$
\int_{1}^{\infty} \int_{0}^{1} \cdots \int_{0}^{1} B_{c^{\prime}}(y)\left(\prod_{j \in J} y_{j}^{j s+s_{j}+z_{j}} \log ^{\alpha_{j}} y_{j}\right)\left(\prod_{j \notin J} y_{j}^{j s+s_{j}}\right) d(\cdots)
$$

converges absolutely whenever $\operatorname{Re} s>-\frac{\operatorname{Re} \Lambda_{j}+s_{j}+1}{j} \geq-\frac{\operatorname{Re} z_{j}+s_{j}+1}{j}$ for $j \in J$, Re $s>-\frac{D_{j}+s_{j}+1}{j}$ for $j \in \bar{J}$ and $\operatorname{Re} s<\frac{L-N-s_{k}-1}{k}$. Since $L$ is arbitrarily large the last condition is superfluous and we can proceed as before.

5.2. Global theory. Let $F$ be a global field. Let $\pi$ be a cuspidal (irreducible) automorphic representation of $G_{k}(\mathbb{A})$. We construct a global integral representation.

Put $n=2 k$ and let $\varphi$ and $\varphi^{\prime}$ be a pair of automorphic forms belonging to the space of the global exceptional representation $\Theta$ of $\widetilde{G}_{n}(\mathbb{A})$ (defined in $\S$ 4.1). The Fourier coefficient $\varphi^{U_{k}, \psi_{k}}(g)$ is a global Shalika functional on $\Theta$. By restriction it is a function on $\widetilde{G_{k}}(\mathbb{A})$, where $G_{k}$ is embedded in $G_{n}$ in the top left block of $M_{k}$. Then $g \mapsto \varphi^{U_{k}, \psi_{k}}(g) \varphi^{\prime U_{k}, \psi_{k}^{-1}}(g)$ is well defined on $G_{k}(\mathbb{A})$ and trivial on $G_{k}(F)$.

Let $f$ be a matrix coefficient of $\pi$, i.e.,

$$
f(g)=\int_{C_{G_{k}}(\mathbb{A}) G_{k}(F) \backslash G_{k}(\mathbb{A})} v(h g) v^{\vee}(h) d h,
$$

where $v\left(\right.$ resp. $\left.v^{\vee}\right)$ is a cusp form in the space of $\pi$ (resp. $\pi^{\vee}$ ). Also let $s \in \mathbb{C}$. Consider the integral

$$
Z\left(f, \varphi^{U_{k}, \psi_{k}}, \varphi^{\prime U_{k}, \psi_{k}^{-1}}, s\right)=\int_{G_{k}(\mathbb{A})} f(g) \varphi^{U_{k}, \psi_{k}}(g) \varphi^{\prime U_{k}, \psi_{k}^{-1}}(g)|\operatorname{det} g|^{s-k / 2} d g .
$$

Theorem 5.4. The integral $Z\left(f, \varphi^{U_{k}, \psi_{k}}, \varphi^{U_{k}, \psi_{k}^{-1}}, s\right)$ satisfies the following properties.

(1) It is absolutely convergent for $\operatorname{Re}(s) \gg 0$. 
(2) For factorizable data $f=\prod_{v} f_{v}, \varphi$ and $\varphi^{\prime}$, write $\varphi^{U_{k}, \psi_{k}}=\prod_{v} \mathscr{S}_{v}$ and $\varphi^{\prime U_{k}, \psi_{k}^{-1}}=\prod_{v} \mathscr{S}_{v}^{\prime}$. In the domain of absolute convergence, the integral is equal to a product

$$
\prod_{v} Z\left(f_{v}, \mathscr{S}_{v}, \mathscr{S}_{v}^{\prime}, s\right)
$$

Moreover, for any sufficiently large finite set of places $S$ of $F$,

$$
Z\left(f, \varphi^{U_{k}, \psi_{k}}, \varphi^{\prime U_{k}, \psi_{k}^{-1}}, s\right)=\prod_{v \in S} Z\left(f_{v}, \mathscr{S}_{v}, \mathscr{S}_{v}^{\prime}, s\right) \frac{L^{S}\left(2 s, \mathrm{Sym}^{2}, \pi\right)}{L^{S}\left(2 s+1, \wedge^{2}, \pi\right)} .
$$

(3) For a suitable choice of data, the integral is not identically zero (as a function of s).

(4) It admits a meromorphic continuation to the complex plane.

Proof. We merely have to establish convergence: the integral is Eulerian by virtue of Theorem 4.22, the other properties follow immediately from the corresponding local results of Theorems 5.1 and 5.3, and the properties of $L^{S}\left(s, \operatorname{Sym}^{2}, \pi\right)$ and $L^{S}\left(s, \wedge^{2}, \pi\right)$.

As in [GJ72, $\S 12$ ], first we show that for $r \gg 0$,

$$
\int_{G_{k}(\mathbb{A})}\left|\varphi^{U_{k}, \psi_{k}}(g) \varphi^{\prime U_{k}, \psi_{k}^{-1}}(g)\right| \cdot|\operatorname{det} g|^{r-k / 2} d g<\infty .
$$

This implies the absolute convergence of $Z\left(f, \varphi^{U_{k}, \psi_{k}}, \varphi^{\prime U_{k}, \psi_{k}^{-1}}, s\right)$, because $|f(g)| \leq C_{f}|\operatorname{det} g|^{r_{0}}$ for some $C_{f}>0$ independent of $g$, and a fixed $r_{0}$ depending only on $\pi$ (when $\pi$ is unitary, $\left.r_{0}=0\right)$. It is enough to consider factorizable data $\varphi$ and $\varphi^{\prime}$. Then we need to bound

$$
\prod_{v} \int_{G_{k}\left(F_{v}\right)}\left|\mathscr{S}_{v}(g) \mathscr{S}_{v}^{\prime}(g)\right| \cdot|\operatorname{det} g|_{v}^{r-k / 2} d_{v} g .
$$

At any $v$, the integral is finite by Theorem 5.1 (11) and Theorem 5.3 (1). Let $S$ be a finite set of places such that for $v \notin S$, all data are unramified. Arguing as in the proof of Theorem 5.1 (44), for $v \notin S$,

$$
\int_{G_{k}\left(F_{v}\right)}\left|\mathscr{S}_{v}(g) \mathscr{S}_{v}^{\prime}(g)\right| \cdot|\operatorname{det} g|_{v}^{r-k / 2} d_{v} g=\sum_{\lambda \in 2 \mathbb{Z}_{+}^{k}} \operatorname{vol}\left(G_{k}\left(\mathcal{O}_{v}\right) \varpi_{v}^{\lambda} G_{k}\left(\mathcal{O}_{v}\right)\right) \delta_{B_{k}}^{1 / 2}\left(\varpi_{v}^{\lambda}\right) q_{v}^{-|\lambda| r} .
$$

Since $\operatorname{vol}\left(G_{k}\left(\mathcal{O}_{v}\right) \varpi_{v}^{\lambda} G_{k}\left(\mathcal{O}_{v}\right)\right) \leq \delta_{B_{k}}^{-1}\left(\varpi_{v}^{\lambda}\right)$, this sum is bounded by

$$
\prod_{i=1}^{k}\left(1-q_{v}^{-2 r-2 i+k+1}\right)^{-1}
$$

According to Weil [Wei95, Chapter VII $\S 1$, Proposition 1] the product of these factors over all the finite places of $F$ is finite for $r \geq(k+1) / 2$, and then

$$
\prod_{v \notin S} \int_{G_{k}\left(F_{v}\right)}\left|\mathscr{S}_{v}(g) \mathscr{S}_{v}^{\prime}(g)\right| \cdot|\operatorname{det} g|_{v}^{r-k / 2} d g<\infty .
$$

Therefore (5.6) is finite, when we take $r \gg(k+1) / 2$ to guarantee the convergence of the integrals also at $v \in S$.

The next theorem will be used in $\S$ 6 to compute the co-period. It relies on the following assumption on $\pi$ and $\Theta$. 
Assumption 5.5. At each Archimedean place $v$ of $F$, there is a neighborhood of $1 / 2$ such that the local zeta integral is holomorphic for all data, i.e., matrix coefficients of $\pi_{v}$ and local metaplectic Shalika functions.

As we show in the proof below, if $\pi$ is unitary with a trivial central character, the analogous assumption at $p$-adic places is valid. This gap will probably be closed once the exponents of $\theta_{n}$ are determined over Archimedean fields; for $n=2$ this can be done directly using the realization of $\theta_{2}$ as a Weil representation and the assumption holds.

Theorem 5.6. Assume that $\pi$ is unitary with a trivial central character, and Assumption 5.5 holds.

(1) The global integral has a pole at $s=1 / 2$ if and only if $L^{S}\left(s, \mathrm{Sym}^{2}, \pi\right)$ has a pole at $s=1$.

(2) For any data $\left(f, \varphi^{U_{k}, \psi_{k}}, \varphi^{\prime U_{k}, \psi_{k}^{-1}}\right)$, there is a punctured neighborhood around $1 / 2$ such that the integral $Z\left(k f, k \varphi^{U_{k}, \psi_{k}}, k \varphi^{\prime U_{k}, \psi_{k}^{-1}}, s\right)$ is holomorphic for all $k \in K$ and $s$ in this neighborhood.

Proof. The first part follows from Theorem 5.4 (2), assuming we can control the integrals at the places in $S$. One can choose data, for which these do not contribute poles or zeros in a neighborhood of $1 / 2$, by Theorem 5.1 (2) and Theorem 5.3 (2). Hence if $L^{S}\left(s, \mathrm{Sym}^{2}, \pi\right)$ has a pole at $s=1$, the integral will have a pole at $s=1 / 2$, for some choice of data. In the other direction, we need to show that the integrals at the places of $S$ cannot contribute any pole. At Archimedean places this is guaranteed by Assumption 5.5. At a $p$-adic place $v \in S$ we can use Theorem 5.1 (5): since $\pi_{v}$ is a local component of a unitary cuspidal automorphic representation of $G_{k}(\mathbb{A})$, it satisfies the prescribed condition on the exponents, by virtue of the results of Luo, Rudnick and Sarnak [LRS99] towards Ramanujan type bounds (see [CKPSS04, p. 221]).

For the second part, first note that if $f$ is a pure tensor, $k$-translations leave most of its components unchanged. The global partial $L$-functions are meromorphic, so one can find a punctured neighborhood of $1 / 2$ where their quotient is holomorphic, and we only have to deal with a finite number of local integrals. At the Archimedean places we again resort to Assumption 5.5. For the remaining $p$-adic places we use Theorem 5.1 (5) and its proof, to choose a neighborhood of $1 / 2$ depending only on the exponents of $\pi_{v}$, at which the local integrals are holomorphic.

Remark 5.7. We mention that over a function field Lafforgue [Laf02a, Laf02b] (following [Dri80, Dri88]) proved the Generalized Ramanujan Conjecture for $G_{k}(\mathbb{A})$, so $\pi_{v}$ is already tempered.

\section{THE CO-PERIOD}

6.1. The co-period integral. Let $F$ be a global field and $\pi$ be a cuspidal self-dual automorphic representation of $G_{k}(\mathbb{A})$ with a trivial central character. Put $n=2 k$ and denote by $A^{+}$the subgroup of idèles of $F$ whose finite components are trivial and Archimedean components are equal and positive.

Let $\rho$ be a smooth complex-valued function on $G_{n}(\mathbb{A}) \times M_{k}(\mathbb{A})$ satisfying the following properties: for $g \in G_{n}(\mathbb{A})$, the function $g \mapsto \rho\left(g, I_{n}\right)$ is right $K$-finite; for any $m, m_{1} \in$ $M_{k}(\mathbb{A})$ and $u \in U_{k}(\mathbb{A}), \rho\left(m u g, m_{1}\right)=\delta_{Q_{k}}^{1 / 2}(m) \rho\left(g, m_{1} m\right)$; and the function $m \mapsto \rho(g, m)$ is a cusp form in the space of $\pi \otimes \pi^{\vee}$. The standard section corresponding to $\rho$ is defined by 
$\rho_{s}\left(m u k, m_{1}\right)=\delta_{Q_{k}}^{s / k}(m) \rho\left(m u k, m_{1}\right)$, for any $k \in K$ and $s \in \mathbb{C}$. The function $\rho_{s}$ belongs to the space of

$$
\operatorname{Ind}_{Q_{k}(\mathbb{A})}^{G_{n}(\mathbb{A})}\left(\delta_{Q_{k}}^{1 / 2+s / k} \pi \otimes \pi^{\vee}\right) .
$$

For simplicity, denote $\rho_{s}(g)=\rho_{s}\left(g, I_{n}\right)$. Form the Eisenstein series

$$
E(g ; \rho, s)=\sum_{g_{0} \in Q_{k}(F) \backslash G_{n}(F)} \rho_{s}\left(g_{0} g\right), \quad g \in G_{n}(\mathbb{A}) .
$$

Let $E_{1 / 2}(g ; \rho)=\operatorname{Res}_{s=1 / 2} E(g ; \rho, s)$ denote the residue of the series at $s=1 / 2$. The residue here is in the sense $\lim _{s \rightarrow 1 / 2}(s-1 / 2) E(g ; \rho, s)$. If we let $\rho$ vary, then $E_{1 / 2}(g ; \rho)$ is nontrivial if and only if $L(\pi \times \pi, s)$ has a pole at $s=1$; equivalently, $L^{S}(\pi \times \pi, s)$ has a pole at $s=1$ for any sufficiently large finite set of places $S$ ([JS81, 1.5 and 3.16]).

Also let $\varphi$ and $\varphi^{\prime}$ be a pair of automorphic forms belonging to the space of the global exceptional representation $\Theta$ of $\widetilde{G}_{n}(\mathbb{A})$. The central character of $\Theta$ is trivial on $\mathfrak{s}\left(C_{G_{n}}^{2}(\mathbb{A})\right)$. The function $g \mapsto \varphi(g) \varphi^{\prime}(g)$ is well defined and moreover, it is left-invariant on a subgroup $C^{\prime}<C_{G_{n}}(\mathbb{A})$ of finite index, which contains $C_{G_{n}}^{2}(\mathbb{A})$ and is also contained in $G_{k}^{\triangle}(\mathbb{A})$ (see [BG92, pp. 159-160]). Specifically, let $S^{\prime}$ be a finite set of places of $F$ such that for all $v \notin S^{\prime}$, $g \mapsto \varphi(g) \varphi^{\prime}(g)$ is right $K_{v}$-invariant, then

$$
C^{\prime}=\left\{z I_{n}: z \in F^{*} \mathbb{A}^{* 2} \prod_{v \notin S^{\prime}} \mathcal{O}_{v}^{*}\right\}
$$

The global co-period integral is

$$
C P\left(E_{1 / 2}(\cdot ; \rho), \varphi, \varphi^{\prime}\right)=\int_{C^{\prime} G(F) \backslash G(\mathbb{A})} E_{1 / 2}(g ; \rho) \varphi(g) \varphi^{\prime}(g) d g .
$$

This integral was defined by Shunsuke Yamana and the first named author (albeit using a slightly different quotient for the integration domain). One may replace $E_{1 / 2}(\cdot ; \rho)$ with any complex-valued function on the quotient, such that the integral is absolutely convergent. As long as $\varphi$ and $\varphi^{\prime}$ are fixed, this does not change the integration domain (in particular, e.g., the co-period is a linear function in the first variable).

Exactly as in Kap15b, since the cuspidal support of $E_{1 / 2}(\cdot ; \rho)$ is $\delta_{Q_{k}}^{-1 /(2 k)} \pi \otimes \pi^{\vee}$ and the only cuspidal exponent of $\Theta$ is $\delta_{B_{n}}^{-1 / 4}$ [KP84, Proposition II.1.2, Theorems II.1.4 and II.2.1], and using [MW95, Lemma I.4.1], one proves the following claim:

Claim 6.1. Integral (6.1) is absolutely convergent.

For the function $\rho$, and with $C^{\prime}$ selected as described above (depending on $\varphi$ and $\varphi^{\prime}$ ) define

$$
f_{\rho}(g)=\int_{C^{\prime} G_{k}^{\Delta}(F) \backslash G_{k}^{\Delta}(\mathbb{A})} \rho\left(I_{n}, c \operatorname{diag}\left(g, I_{k}\right)\right) d c, \quad g \in G_{k}(\mathbb{A}) .
$$

As noted above, $C^{\prime}<G_{k}^{\triangle}(\mathbb{A})$. Then $f_{\rho}$ is a matrix coefficient of $\pi$. Since the integrand is left-invariant on $C_{G_{n}}(\mathbb{A})$, taking a different $C^{\prime}$ only multiplies $f_{\rho}$ by some nonzero constant.

Theorem 6.2. Assume $F$ is a function field or Assumption 5.5 holds. The co-period (6.1) satisfies the following properties.

(1) $C P\left(E_{1 / 2}(\cdot ; \rho), \varphi, \varphi^{\prime}\right)=\int_{K} \operatorname{Res}_{s=1 / 2} Z\left(f_{k \rho}, k \varphi^{U_{k}, \psi_{k}}, k \varphi^{\prime U_{k}, \psi_{k}^{-1}}, s\right) d k$. 
(2) It is nonzero for some choice of data $\left(\rho, \varphi, \varphi^{\prime}\right)$ if and only if

$$
\operatorname{Res}_{s=1 / 2} Z\left(f_{\rho_{1}}, \varphi_{1}^{U_{k}, \psi_{k}}, \varphi_{1}^{\prime U_{k}, \psi_{k}^{-1}}, s\right)
$$

does not vanish for some matrix coefficient $f_{\rho_{1}}$ of $\pi$ and automorphic forms $\varphi_{1}, \varphi_{1}^{\prime}$ in the space of $\Theta$.

(3) The co-period is nonzero if and only if $L^{S}\left(s, \mathrm{Sym}^{2}, \pi\right)$ has a pole at $s=1$.

Remark 6.3. Part (3) was obtained by Shunsuke Yamana and the first named author, it is included here because we can also deduce it from Theorem 5.6.

Proof. We apply the truncation operator of Arthur [Art80, Art78] to $E(g ; \rho, s)$ as in, e.g., JR92, Jia98, GRS99b, GJR01, Kap15b. For a real number $d>1$, let $c h_{>d}: \mathbb{R}_{>0} \rightarrow\{0,1\}$ be the characteristic function of $\mathbb{R}_{>d}$ and set $c h_{\leq d}=1-c h_{>d}$. Also extend $\delta_{Q_{k}}$ to $G_{n}(\mathbb{A})$ via the Iwasawa decomposition. The truncation operator is given by

$$
\Lambda_{d} E(g ; \rho, s)=E(g ; \rho, s)-\sum_{g_{0} \in Q_{k}(F) \backslash G_{n}(F)} E^{U_{k}}\left(g_{0} g ; \rho, s\right) c h_{>d}\left(\delta_{Q_{k}}\left(g_{0} g\right)\right),
$$

where $E^{U_{k}}$ is the constant term of the series along $U_{k}$. Since $\pi$ is cuspidal, $E^{U_{k}}(g ; \rho, s)=$ $\rho_{s}(g)+M(w, s) \rho_{s}(g)$. Here $M(w, s)$ is the global intertwining operator corresponding to a Weyl element $w$ taking $U_{k}$ to $U_{k}^{-}$. Write

$$
\Lambda_{d} E(g ; \rho, s)=\mathcal{E}_{1}^{d}(g ; s)-\mathcal{E}_{2}^{d}(g ; s),
$$

an equality between meromorphic functions in $s$, where

$$
\begin{aligned}
& \mathcal{E}_{1}^{d}(g ; s)=\sum_{g_{0} \in Q_{k}(F) \backslash G_{n}(F)} \rho_{s}\left(g_{0} g\right) c h_{\leq d}\left(\delta_{Q_{k}}\left(g_{0} g\right)\right), \\
& \mathcal{E}_{2}^{d}(g ; s)=\sum_{g_{0} \in Q_{k}(F) \backslash G_{n}(F)} M(w, s) \rho_{s}\left(g_{0} g\right) c h_{>d}\left(\delta_{Q_{k}}\left(g_{0} g\right)\right) .
\end{aligned}
$$

Claim 6.4. Fix $d>1$. The integral obtained from (6.1) by replacing $E_{1 / 2}(\cdot ; \rho)$ with $\mathcal{E}_{j}^{d}(\cdot ; s)$ and taking the absolute value of $\rho_{s}\left(M(w, s) \rho_{s}\right.$ for $\left.j=2\right), \varphi$ and $\varphi^{\prime}$, is finite for $\operatorname{Re}(s) \gg 0$.

It follows that for fixed $d>1$ and $\operatorname{Re}(s) \gg 0, C P\left(\Lambda_{d} E(\cdot ; \rho, s), \varphi, \varphi^{\prime}\right)$ is absolutely convergent and

$$
C P\left(\Lambda_{d} E(\cdot ; \rho, s), \varphi, \varphi^{\prime}\right)=C P\left(\mathcal{E}_{1}^{d}(\cdot ; s), \varphi, \varphi^{\prime}\right)-C P\left(\mathcal{E}_{2}^{d}(\cdot ; s), \varphi, \varphi^{\prime}\right) .
$$

The heart of the argument is in the following result.

Proposition 6.5. Assume $d$ is sufficiently large. The integrals $C P\left(\mathcal{E}_{j}^{d}(\cdot ; s), \varphi, \varphi^{\prime}\right)$ admit meromorphic continuation to the plane. There exist complex-valued functions $\alpha_{0}(s), \alpha_{1}(s, d)$, $\alpha_{2}(s)$ and $\alpha_{3}(d, s)$ such that

$$
\begin{aligned}
& C P\left(\mathcal{E}_{1}^{d}(\cdot ; s), \varphi, \varphi^{\prime}\right)=\frac{d^{s / k}}{s k} \alpha_{0}(s)+\alpha_{1}(d, s), \\
& C P\left(\mathcal{E}_{2}^{d}(\cdot ; s), \varphi, \varphi^{\prime}\right)=\frac{d^{-s / k}}{s k} \alpha_{2}(s)+\alpha_{3}(d, s) .
\end{aligned}
$$

The function $\alpha_{0}(s)$ is entire; for $j>0$ the functions $\alpha_{j}(\cdots)$ are meromorphic in $s$ (for fixed $d$ if $j=1,3)$;

$$
\operatorname{Res}_{s=1 / 2} \alpha_{1}(d, s)=\int_{K} \operatorname{Res}_{s=1 / 2} Z\left(f_{k \rho}, k \varphi^{U_{k}, \psi_{k}}, k \varphi^{U_{k}, \psi_{k}^{-1}}, s\right) d k
$$


$\alpha_{2}(s)$ has at most a simple pole at $s=1 / 2$, and

$$
\lim _{d \rightarrow \infty} \operatorname{Res}_{s=1 / 2} \alpha_{3}(d, s)=0
$$

Furthermore, when $F$ is a number field, $\alpha_{0}(s)=\alpha_{0}$ is a constant, and if $F$ is a function field, we in fact have $C P\left(\mathcal{E}_{2}^{d}(\cdot ; s), \varphi, \varphi^{\prime}\right)=0$.

Exactly as argued in [Kap15b, pp. 14-15], the properties of the truncated series imply that when we take the residue in (6.2),

$$
C P\left(\Lambda_{d} E_{1 / 2}(\cdot ; \rho), \varphi, \varphi^{\prime}\right)=\operatorname{Res}_{s=1 / 2} C P\left(\mathcal{E}_{1}^{d}(\cdot ; s), \varphi, \varphi^{\prime}\right)-\operatorname{Res}_{s=1 / 2} C P\left(\mathcal{E}_{2}^{d}(\cdot ; s), \varphi, \varphi^{\prime}\right) .
$$

According to Labesse and Waldspurger [LW13, Proposition 4.3.3], $\Lambda_{d} E_{1 / 2}(g ; \rho)$ is bounded by a sum $E_{1 / 2}(g ; \rho)+\vartheta(g, d ; \rho)$, where $\vartheta(g, c ; \rho)$ is uniformly rapidly decreasing in $d$ and rapidly decreasing in $g$. Since $\left|\Lambda_{d} E(\cdot ; \rho, s)\right| \leq|E(\cdot ; \rho, s)|$, Claim 6.1 and the Dominated Convergence Theorem imply that we can take the limit $d \rightarrow \infty$ under the integral sign on the left-hand side of the last equality. Then according to the proposition,

$$
C P\left(E_{1 / 2}(\cdot ; \rho), \varphi, \varphi^{\prime}\right)=\int_{K} \operatorname{Res}_{s=1 / 2} Z\left(f_{k \rho}, k \varphi^{U_{k}, \psi_{k}}, k \varphi^{\prime U_{k}, \psi_{k}^{-1}}, s\right) d k .
$$

This completes part (1) of the theorem.

The $d k$-integral has a pole at $s=1 / 2$ for some choice of data $\left(\rho, \varphi, \varphi^{\prime}\right)$ if and only if $\operatorname{Res}_{s=1 / 2} Z(\cdots) \neq 0$, for some choice of data $\left(f_{\rho_{1}}, \varphi_{1}, \varphi_{1}^{\prime}\right)$. This follows exactly as in GJR01, Theorem 3.2] (see also [JR92, Proposition 2] and [Jia98]), their arguments are general and apply to our setting. The proof of the second part of the theorem is complete. The third part follows immediately from the second and Theorem 5.6 (11).

Proof of Claim 6.4. Collapsing the summation of the Eisenstein series into the integral and using the Iwasawa decomposition we obtain

$$
\begin{aligned}
& \int_{K} \int_{C^{\prime} M_{k}(F) \backslash M_{k}(\mathbb{A})}\left|\rho_{s}\right|(m k) c h_{\leq d}\left(\delta_{Q_{k}}(m)\right) \delta_{Q_{k}}^{r / k-1 / 2}(m) \\
& \times \int_{U_{k}(F) \backslash U_{k}(\mathbb{A})}\left|\varphi \varphi^{\prime}\right|(u m k) d u d m d k,
\end{aligned}
$$

where $r=\operatorname{Re}(s)$. This is finite for $r \gg 0$, because $\varphi$ and $\varphi^{\prime}$ have moderate growth, and $m \mapsto \rho(m)$ is a cusp form.

Proof of Proposition 6.5 - number field case. First consider $C P\left(\mathcal{E}_{1}^{d}(\cdot ; s), \varphi, \varphi^{\prime}\right)$. We take $s$ in the domain of absolute convergence, where our integral manipulations are justified. Collapsing the summation and using the Iwasawa decomposition,

$$
\begin{aligned}
C P\left(\mathcal{E}_{1}^{d}(\cdot ; s), \varphi, \varphi^{\prime}\right)= & \int_{K} \int_{C^{\prime} M_{k}(F) \backslash M_{k}(\mathbb{A})} \rho_{s}(m k) c h_{\leq d}\left(\delta_{Q_{k}}(m)\right) \\
& \times \int_{U_{k}(F) \backslash U_{k}(\mathbb{A})} \varphi(u m k) \varphi^{\prime}(u m k) d u \delta_{Q_{k}}^{-1}(m) d m d k .
\end{aligned}
$$

Next we appeal to the results of $\S 4.3$ and replace $\varphi$ with its Fourier expansion along $U_{k}$. In the notation of $\S 4.3$, the integral becomes a sum of integrals $\sum_{j=0}^{k} \mathrm{I}_{j}$, where

$$
\mathrm{I}_{j}=\int_{K} \int_{C^{\prime} \mathrm{St}_{n, k}\left(\psi_{j}\right)(F) \backslash M_{k}(\mathbb{A})} \rho_{s}(m k) c h_{\leq d}\left(\delta_{Q_{k}}(m)\right) \varphi^{U_{k}, \psi_{j}}(m k) \varphi^{\prime U_{k}, \psi_{j}^{-1}}(m k) \delta_{Q_{k}}^{-1}(m) d m d k .
$$


This formal manipulation is justified using the fact that $\varphi$ has uniform moderate growth. See Kap15b, p. 17] for the complete argument leading to the sum of integrals $\mathrm{I}_{j}$, the identities here are very similar (and simpler). By virtue of Proposition 4.6, the integrals $\mathrm{I}_{j}$ with $0<j<k$ vanish, because we may introduce an inner integration $\int_{V_{j}(F) \backslash V_{j}(\mathbb{A})} \rho_{s}(v g) d v$ along a unipotent subgroup $V_{j}$ of $M_{k}$, which vanishes because $m \mapsto \rho(m)$ is a cusp form.

Consider $\mathrm{I}_{0}$. Then $\psi_{0}=1$ and $\operatorname{St}_{n, k}\left(\psi_{0}\right)=M_{k}$. For a subgroup $H<M_{k}(\mathbb{A})$, let $H^{1}=$ $\left\{h \in H:\left|\delta_{Q_{k}}(h)\right|=1\right\}$. Since $C^{\prime} M_{k}(F)<M_{k}(\mathbb{A})^{1}$ and

$$
M_{k}(\mathbb{A})=M_{k}(\mathbb{A})^{1} \times\left\{\operatorname{diag}\left(t I_{k}, I_{k}\right): t \in A^{+}\right\},
$$

we obtain

$$
\begin{aligned}
\mathrm{I}_{0}= & \int_{K} \int_{C^{\prime} M_{k}(F) \backslash M_{k}(\mathbb{A})^{1}} \int_{\mathbb{R}_{>0}} \rho_{s} \varphi^{U_{k}} \varphi^{\prime U_{k}}\left(\operatorname{diag}\left(t I_{k}, I_{k}\right) m k\right) \\
& \times c h_{\leq d}\left(t^{k^{2}}\right) \delta_{Q_{k}}^{-1}\left(\operatorname{diag}\left(t I_{k}, I_{k}\right)\right) t^{-1} d t d m d k .
\end{aligned}
$$

Here we identified $A^{+}$with $\mathbb{R}_{>0}, d t$ is the standard Lebesgue measure on $\mathbb{R}$ and the constant normalizing the measure $d m$ on the right-hand side is omitted. For $m \in M_{k}(\mathbb{A})^{1}$,

$$
\rho_{s}\left(\operatorname{diag}\left(t I_{k}, I_{k}\right) m k\right)=\delta_{Q_{k}}^{1 / 2+s / k}\left(\operatorname{diag}\left(t I_{k}, I_{k}\right)\right) \rho(m k) .
$$

By virtue of Theorem 4.8, the function $\varphi^{U_{k}}$ on $\widetilde{M}_{k}(\mathbb{A})$ belongs to the space of $\delta_{Q_{k}}^{1 / 4} \Theta_{M_{k}}$, where $\Theta_{M_{k}}$ corresponds to a character which is a lift of $\left.\left(\delta_{B_{k}}^{1 / 4} \otimes \delta_{B_{k}}^{1 / 4}\right)\right|_{T_{n}^{2}}$ (the chosen Borel subgroup of $M_{k}$ is $\left.B_{k} \times B_{k}\right)$. Therefore

$$
\varphi^{U_{k}} \varphi^{\prime U_{k}}\left(\operatorname{diag}\left(t I_{k}, I_{k}\right) m k\right)=\delta_{Q_{k}}^{1 / 2}\left(\operatorname{diag}\left(t I_{k}, I_{k}\right)\right) \varphi^{U_{k}} \varphi^{\prime U_{k}}(m k),
$$

Combining (6.3) and (6.4) we obtain an inner integral

$$
\int_{0<t^{k^{2}} \leq d} t^{s k-1} d t=\frac{d^{s / k}}{s k}
$$

multiplied by a $d m d k$-integral, which is independent of $s$ and $d$. Therefore $\mathrm{I}_{0}=\frac{d^{s} / k}{s k} \alpha_{0}$.

Regarding $\mathrm{I}_{k}, \psi_{k}$ is the Shalika character and $\operatorname{St}_{n, k}\left(\psi_{k}\right)=G_{k}^{\triangle}$. According to Theorem 4.22,

$$
\varphi^{U_{k}, \psi_{k}} \varphi^{\prime U_{k}, \psi_{k}^{-1}}\left(c^{\triangle} m k\right)=\varphi^{U_{k}, \psi_{k}} \varphi^{\prime U_{k}, \psi_{k}^{-1}}(m k), \quad \forall c \in G_{k}(\mathbb{A}) .
$$

Therefore we may introduce the inner integration given by the formula for $f_{\rho}$ and

$$
\mathrm{I}_{k}=\int_{K} \int_{G_{k}(\mathbb{A})} f_{k \rho}(g) \varphi^{U_{k}, \psi_{k}} \varphi^{\prime U_{k}, \psi_{k}^{-1}}\left(\operatorname{diag}\left(g, I_{k}\right) k\right)|\operatorname{det} g|^{s-k / 2} c h_{\leq d}\left(|\operatorname{det} g|^{k}\right) d g d k .
$$

Consider the inner integral, for a fixed $k \in K$. Without the function $c h_{\leq d}$, it is the integral $Z=Z\left(f_{k \rho}, k \varphi^{U_{k}, \psi_{k}}, k \varphi^{\prime U_{k}, \psi_{k}^{-1}}, s\right)$ given by (15.4). Denote by $Z_{\leq d}$ (resp. $Z_{>d}$ ) the integral obtained when the integrand is multiplied by $c h_{\leq d}\left(\operatorname{resp} . c h_{>d}\right)$. Then for $\operatorname{Re}(s) \gg 0$, $Z=Z_{\leq d}+Z_{>d}$. The following claim is the main observation needed to complete the proof.

Claim 6.6. Fix d, sufficiently large. For any $s, Z_{>d}$ is absolutely convergent, uniformly for $s$ in compact subsets of $\mathbb{C}$. Hence $Z_{>d}$ has a holomorphic continuation to the plane. Moreover for any fixed $s, Z_{>d}=0$ as $d \rightarrow \infty$. 
The proof will be given below. Assume that $d$ is large enough. By Theorem 5.4 (4), $Z$ admits a meromorphic continuation to $\mathbb{C}$, and therefore the claim implies that $Z_{\leq d}$ has a meromorphic continuation to the plane. Furthermore, because $Z_{>d}$ is holomorphic, we deduce that for any $s_{0}, \operatorname{Res}_{s=s_{0}} Z=\operatorname{Res}_{s=s_{0}} Z_{\leq d}$, and since the left-hand side is independent of $d$, the residues of $Z_{\leq d}$ are also independent of $d$.

Because $K$ is compact, $\mathrm{I}_{k}$ also has a meromorphic continuation. It follows that

$$
\operatorname{Res}_{s=1 / 2} \mathrm{I}_{k}=\operatorname{Res}_{s=1 / 2} \int_{K} Z_{\leq d}\left(f_{k \rho}, k \varphi^{U_{k}, \psi_{k}}, k \varphi^{\prime U_{k}, \psi_{k}^{-1}}, s\right) d k .
$$

According to Theorem 5.6 (2) and its proof, at $s=1 / 2$ the integral $Z$ has at most a simple pole, and there is a punctured neighborhood of $1 / 2$ where the integral is holomorphic for all $k \in K$. The same applies to $Z_{\leq d}$, hence by virtue of Cauchy's Residue Theorem and Fubini's Theorem we can take the residue under the integral sign and obtain

$$
\begin{aligned}
& \int_{K} \operatorname{Res}_{s=1 / 2} Z_{\leq d}\left(f_{k \rho}, k \varphi^{U_{k}, \psi_{k}}, k \varphi^{\prime U_{k}, \psi_{k}^{-1}}, s\right) d k \\
& =\int_{K} \operatorname{Res}_{s=1 / 2} Z\left(f_{k \rho}, k \varphi^{U_{k}, \psi_{k}}, k \varphi^{\prime U_{k}, \psi_{k}^{-1}}, s\right) d k .
\end{aligned}
$$

The right-hand side is independent of $d$. Hence $\mathrm{I}_{k}$ qualifies as the function $\alpha_{1}(s, d)$. In addition, now that we proved that both $\mathrm{I}_{0}$ and $\mathrm{I}_{k}$ admit meromorphic continuations, the same holds for $C P\left(\mathcal{E}_{1}^{d}(\cdot ; s), \varphi, \varphi^{\prime}\right)$.

Next we handle $C P\left(\mathcal{E}_{2}^{d}(\cdot ; s), \varphi, \varphi^{\prime}\right)$. We may proceed as above and obtain a sum of two integrals $\mathrm{I}_{0}+\mathrm{I}_{k}$, where $\rho_{s}$ and $c h_{\leq d}$ are replaced by $M(w, s) \rho_{s}$ and $c h>d$. Now $\mathrm{I}_{0}$ takes the form

$$
\begin{aligned}
\mathrm{I}_{0}= & \int_{K} \int_{C^{\prime} M_{k}(F) \backslash M_{k}(\mathbb{A})^{1}} \int_{\mathbb{R}_{>0}} M(w, s) \rho_{s} \varphi^{U_{k}} \varphi^{\prime U_{k}}\left(\operatorname{diag}\left(t I_{k}, I_{k}\right) m k\right) \\
& \times c h_{>d}\left(t^{k^{2}}\right) \delta_{Q_{k}}^{-1}\left(\operatorname{diag}\left(t I_{k}, I_{k}\right)\right) t^{-1} d t d m d k
\end{aligned}
$$

Since

$$
M(w, s) \rho_{s}\left(\operatorname{diag}\left(t I_{k}, I_{k}\right) m k\right)=\delta_{Q_{k}}^{1 / 2-s / k}\left(\operatorname{diag}\left(t I_{k}, I_{k}\right)\right) M(w, s) \rho_{s}(m k)
$$

the inner integral in this case becomes

$$
\int_{d<t^{k^{2}<\infty}} t^{-s k-1} d t
$$

Thus

$$
\mathrm{I}_{0}=\frac{d^{-s / k}}{s k} \int_{K} \int_{C^{\prime} M_{k}(F) \backslash M_{k}(\mathbb{A})^{1}} M(w, s) \rho_{s} \varphi^{U_{k}} \varphi^{\prime U_{k}}(m k) d m d k .
$$

The $d m d k$-integral still depends on $s$, because of the intertwining operator, but it is a meromorphic function in $s$ and if it has a pole at $s=1 / 2$, this pole is simple. Hence the constant $\alpha_{0}$ is replaced by a function $\alpha_{2}(s)$ with the stated properties.

Regarding $\mathrm{I}_{k}$, with the above notation

$$
\mathrm{I}_{k}=\int_{K} Z_{>d}\left(f_{k M(w, s) \rho_{s}}, k \varphi^{U_{k}, \psi_{k}}, k \varphi^{\prime U_{k}, \psi_{k}^{-1}},-s\right) d k .
$$

Note that since $m \mapsto \rho(g, m)$ is a cusp form, the matrix coefficient $f_{M(w, s) \rho_{s}}$ is a meromorphic function in $s$ and $\operatorname{Res}_{s=1 / 2} f_{M(w, s) \rho_{s}}=f_{\operatorname{Res}_{s=1 / 2} M(w, s) \rho_{s}}$. The convergence properties of $Z_{>d}$ 
stated above, imply that $\mathrm{I}_{k}$ admits a meromorphic continuation whose poles are contained in the set of poles of $M(w, s)$, and also

$$
\operatorname{Res}_{s=1 / 2} \mathrm{I}_{k}=\int_{K} Z_{>d}\left(f_{\operatorname{Res}_{s=1 / 2} k M(w, s) \rho_{s}}, k \varphi^{U_{k}, \psi_{k}}, k \varphi^{\prime U_{k}, \psi_{k}^{-1}},-s\right) d k .
$$

Hence when we let $d \rightarrow \infty$ Res $_{s=1 / 2} \mathrm{I}_{k}=0$.

Proof of Claim 6.6. Consider the function $\phi(g)=\left|\varphi^{U_{k}, \psi_{k}} \varphi^{\prime U_{k}, \psi_{k}^{-1}}\left(\operatorname{diag}\left(g, I_{k}\right)\right)\right|$ on $G_{k}(\mathbb{A})$. The global matrix coefficient $f_{\rho}$ is bounded, therefore it is enough to establish the stated convergence properties for an integral of the form

$$
\int_{G_{k}(\mathbb{A})} \phi(g) c h_{>d}(|\operatorname{det} g|)|\operatorname{det} g|^{r} d g=\int_{G_{k}(\mathbb{A})^{1}} \int_{\mathbb{R}_{>d^{1 / k}}} \phi(g t) t^{k r-1} d t d g, \quad r \in \mathbb{R} .
$$

But according to (5.5), for $d>1$, this integral is finite even for $r \gg 0$.

Proof of Proposition 6.5 - function field case. We argue as in the case of a number field and use similar notation, but the proof is greatly simplified. We write $C P\left(\mathcal{E}_{1}^{d}(\cdot ; s), \varphi, \varphi^{\prime}\right)$ as a sum of integrals, the only surviving ones are $\mathrm{I}_{0}$ and $\mathrm{I}_{k}$. We can then write $\mathrm{I}_{0}$ in the form

$$
\begin{aligned}
\mathrm{I}_{0}= & \int_{K} \int_{\left(C_{G_{k}}(\mathbb{A}) C^{\prime} M_{k}(F)\right) \backslash M_{k}(\mathbb{A})} \int_{C_{G_{k}}(F) \backslash C_{G_{k}}(\mathbb{A})} \rho_{s} \varphi^{U_{k}} \varphi^{\prime U_{k}}(z m k) \\
& \times c h_{\leq d}\left(\delta_{Q_{k}}(z m)\right) \delta_{Q_{k}}^{-1}(z m) d z d m d k .
\end{aligned}
$$

Since over a function field, a cusp form on $G_{k}(\mathbb{A})$ is compactly supported modulo $C_{G_{k}}(\mathbb{A}) G_{k}(F)$, $\mathrm{I}_{0}$ becomes a linear combination of integrals of the form

$$
\int_{F^{*} \backslash \mathbb{A}^{*}} \rho_{s} \varphi^{U_{k}} \varphi^{\prime U_{k}}\left(\operatorname{diag}\left(t I_{k}, I_{k}\right)\right) c h_{\leq d}\left(t^{k^{2}}\right) \delta_{Q_{k}}^{-1}\left(\operatorname{diag}\left(t I_{k}, I_{k}\right)\right) d t
$$

Set $\left(\mathbb{A}^{*}\right)^{1}=\left\{a \in \mathbb{A}^{*}:|a|=1\right\}$ and identify $\left(\mathbb{A}^{*}\right)^{1} \backslash \mathbb{A}^{*}$ with $\mathbb{R}_{>0}$. Since (6.3) and (6.4) still hold, the integrand is invariant on $\left\{\operatorname{diag}\left(t I_{k}, I_{k}\right): t \in\left(\mathbb{A}^{*}\right)^{1}\right\}$. Thus we see that (6.6) equals (6.5) multiplied by the volume of $F^{*} \backslash\left(\mathbb{A}^{*}\right)^{1}$ (which is finite).

Next note that according to Claim [3.2, the projection of the support of $\varphi^{U_{k}, \psi_{k}}$ on $G_{k}(\mathbb{A})$ is contained in the support of some $\phi \in \mathcal{S}\left(\mathbb{A}_{k \times k}\right)$. Hence if $g \in G_{k}(\mathbb{A})$ satisfies $|\operatorname{det} g|^{k}>d$ for a large enough $d$ (depending only on $\varphi), \varphi^{U_{k}, \psi_{k}} \varphi^{\prime U_{k}, \psi_{k}^{-1}}\left(\operatorname{diag}\left(g, I_{k}\right)\right)=0$. This means that the integrals $Z_{>d}$ vanish identically. We can conclude that $\mathrm{I}_{k}$ satisfies the stated properties and moreover, $C P\left(\mathcal{E}_{2}^{d}(\cdot ; s), \varphi, \varphi^{\prime}\right)=0$, because in the corresponding summands $\mathrm{I}_{0}$ and $\mathrm{I}_{k}$ appears $c h_{>d}$.

\section{REFEREnCES}

[AG94] A. Ash and D. Ginzburg. p-adic L-functions for GL(2n). Invent. Math., 116(1-3):27-73, 1994.

[AGJ09] A. Aizenbud, D. Gourevitch, and H. Jacquet. Uniqueness of Shalika functionals: the Archimedean case. Pacific J. Math., 243(2):201-212, 2009.

[Art78] J. G. Arthur. A trace formula for reductive groups. I. Terms associated to classes in $G(\mathbf{Q})$. Duke Math. J., 45(4):911-952, 1978.

[Art80] J. Arthur. A trace formula for reductive groups. II. Applications of a truncation operator. Compositio Math., 40(1):87-121, 1980.

[Ban98] W. Banks. A corollary to Bernstein's theorem and Whittaker functionals on the metaplectic group. Math. Res. Letters, 5(6):781-790, 1998.

[BB06] J. Beineke and D. Bump. A summation formula for divisor functions associated to lattices. Forum Math., 18(6):869-906, 2006. 
[BF90] D. Bump and S. Friedberg. The exterior square automorphic $L$-functions on GL(n). In Festschrift in honor of I. I. Piatetski-Shapiro on the occasion of his sixtieth birthday, Part II (Ramat Aviv, 1989), volume 3 of Israel Math. Conf. Proc., pages 47-65. Weizmann, Jerusalem, 1990.

[BFG03] D. Bump, S. Friedberg, and D. Ginzburg. Small representations for odd orthogonal groups. Internat. Math. Res. Notices, 2003(25):1363-1393, 2003.

[BFH91] D. Bump, S. Friedberg, and J. Hoffstein. p-adic Whittaker functions on the metaplectic group. Duke Math. J., 63(2):379-397, 1991.

[BG92] D. Bump and D. Ginzburg. Symmetric square L-functions on GL(r). Ann. of Math. (2), 136(1):137-205, 1992.

[BJ13] D. Ban and C. Jantzen. The Langlands quotient theorem for finite central extensions of $p$-adic groups. Glas. Mat. Ser. III, 48(2):313-334, 2013.

[BLS99] W. D. Banks, J. Levy, and M. R. Sepanski. Block-compatible metaplectic cocycles. J. Reine Angew. Math., 1999(507):131-163, 1999.

[BW80] A. Borel and N. R. Wallach. Continuous cohomology, discrete subgroups, and representations of reductive groups, volume 94 of Annals of Mathematics Studies. Princeton University Press, Princeton, N.J., 1980.

[BZ76] I. N. Bernstein and A. V. Zelevinsky. Representations of the group $G L(n, F)$ where $F$ is a local non-Archimedean field. Russian Math. Surveys, 31(3):1-68, 1976.

[BZ77] I. N. Bernstein and A. V. Zelevinsky. Induced representations of reductive $p$-adic groups I. Ann. Scient. Éc. Norm. Sup., 10(4):441-472, 1977.

[Car93] R. W. Carter. Finite groups of Lie type. Wiley Classics Library. John Wiley \& Sons Ltd., Chichester, 1993. Conjugacy classes and complex characters, Reprint of the 1985 original, A Wiley-Interscience Publication.

[Cas80] W. Casselman. The unramified principal series of $p$-adic groups I: the spherical function. Compositio Math., 40(3):387-406, 1980.

[Cas95] W. Casselman. Introduction to the theory of admissible representations of $p$-adic reductive groups, 1995. preprint, http://www.math.ubc.ca/ cass/research/pdf/p-adic-book.pdf.

[CKPSS04] J. W. Cogdell, H.H. Kim, I. I. Piatetski-Shapiro, and F. Shahidi. Functoriality for the classical groups. Publ. Math. IHES., 99:163-233, 2004.

[CM93] D. H. Collingwood and W. M. McGovern. Nilpotent orbits in semisimple Lie algebras. Van Nostrand Reinhold Mathematics Series. Van Nostrand Reinhold Co., New York, 1993.

[CO13] G. Chinta and O. Offen. A metaplectic Casselman-Shalika formula for $\mathrm{GL}_{r}$. Amer. J. Math., 135(2):403-441, 2013.

[CS80] W. Casselman and J. A. Shalika. The unramified principal series of $p$-adic groups II: the Whittaker function. Compositio Math., 41(2):207-231, 1980.

[DAV86] Jr. D. A. Vogan. The unitary dual of GL $(n)$ over an Archimedean field. Invent. Math., 83(3):449505, 1986.

[Dri80] V. G. Drinfel'd. Langlands' conjecture for GL(2) over functional fields. In Proceedings of the International Congress of Mathematicians (Helsinki, 1978), pages 565-574. Acad. Sci. Fennica, Helsinki, 1980.

[Dri88] V. G. Drinfel'd. Proof of the Petersson conjecture for GL(2) over a global field of characteristic p. Funktsional. Anal. i Prilozhen., 22(1):34-54, 96, 1988.

[FJ93] S. Friedberg and H. Jacquet. Linear periods. J. Reine Angew. Math., 1993(443):91-139, 1993.

[FK86] Y. Z. Flicker and D. A. Kazhdan. Metaplectic correspondence. Inst. Hautes Études Sci. Publ. Math., 64(1):53-110, 1986.

[Gin90] D. Ginzburg. L-functions for $S O_{n} \times G L_{k}$. J. Reine Angew. Math., 405:156-180, 1990 .

[Gin06] D. Ginzburg. Certain conjectures relating unipotent orbits to automorphic representations. Israel J. Math., 151(1):323-355, 2006.

[GJ72] R. Godement and H. Jacquet. Zeta functions of simple algebras, volume 260 of Lecture Notes in Math. Springer-Verlag, Berlin, 1972.

[GJR01] D. Ginzburg, D. Jiang, and S. Rallis. Nonvanishing of the central critical value of the third symmetric power L-functions. Forum Math., 13(1):109-132, 2001.

[GJR04] D. Ginzburg, D. Jiang, and S. Rallis. On the nonvanishing of the central value of the RankinSelberg L-functions. J. Amer. Math. Soc., 17(3):679-722 (electronic), 2004. 
[GJS10] D. Ginzburg, D. Jiang, and D. Soudry. Periods of automorphic forms, poles of $L$-functions and functorial lifting. Sci. China Math., 53(9):2215-2238, 2010.

[GK75] I. M. Gel'fand and D. A. Kajdan. Representations of the group $\operatorname{GL}(n, K)$ where $K$ is a local field. In Lie groups and their representations (Proc. Summer School, Bolyai János Math. Soc., Budapest, 1971), pages 95-118. Halsted, New York, 1975.

[GPSR87] S. Gelbart, I. I. Piatetski-Shapiro, and S. Rallis. L-Functions for $G \times G L(n)$, volume 1254 of Lecture Notes in Math. Springer-Verlag, New York, 1987.

[GPSR97] D. Ginzburg, I. I. Piatetski-Shapiro, and S. Rallis. L-functions for the orthogonal group, volume 128, no. 611 of Mem. Amer. Math. Soc. Amer. Math. Soc., Providence, R. I., 1997.

[GRS98] D. Ginzburg, S. Rallis, and D. Soudry. L-functions for symplectic groups. Bull. Soc. math. France, 126:181-244, 1998.

[GRS99a] D. Ginzburg, S. Rallis, and D. Soudry. On a correspondence between cuspidal representations of $G L_{2 n}$ and $\widetilde{S p}_{2 n}$. J. Amer. Math. Soc., 12(3):849-907, 1999.

[GRS99b] D. Ginzburg, S. Rallis, and D. Soudry. On explicit lifts of cusp forms from $\mathrm{GL}_{m}$ to classical groups. Ann. of Math. (2), 150(3):807-866, 1999.

[GRS01] D. Ginzburg, S. Rallis, and D. Soudry. Generic automorphic forms on $S O(2 n+1)$ : functorial lift to $G L(2 n)$, endoscopy, and base change. Internat. Math. Res. Notices., 2001(14):729-764, 2001.

[GRS11] D. Ginzburg, S. Rallis, and D. Soudry. The Descent Map from Automorphic Representations of GL(n) to Classical Groups. World Scientific Publishing, Singapore, 2011.

[Hir99] Y. Hironaka. Spherical functions and local densities on Hermitian forms. J. Math. Soc. Japan, 51(3):553-581, 1999.

[Jia98] D. Jiang. $G_{2}$-periods and residual representations. J. Reine Angew. Math., 497:17-46, 1998.

[JNQ08] D. Jiang, C. Nien, and Y. Qin. Local Shalika models and functoriality. Manuscripta Math., 127(2):187-217, 2008.

[JNQ10] D. Jiang, C. Nien, and Y. Qin. Symplectic supercuspidal representations of GL $(2 n)$ over $p$-adic fields. Pacific J. Math., 245(2):273-313, 2010.

[JQ07] D. Jiang and Y. Qin. Residues of Eisenstein series and generalized Shalika models for $\mathrm{SO}_{4 n} . J$. Ramanujan Math. Soc., 22(2):101-133, 2007.

[JR92] H. Jacquet and S. Rallis. Symplectic periods. J. Reine Angew. Math., 1992(423):175-197, 1992.

[JR96] H. Jacquet and S. Rallis. Uniqueness of linear periods. Compositio Math., 102(1):65-123, 1996.

[JS81] H. Jacquet and J. A. Shalika. On Euler products and the classification of automorphic representations I. Amer. J. Math., 103(3):499-558, 1981.

[JS90] H. Jacquet and J. Shalika. Exterior square L-functions. In Automorphic forms, Shimura varieties, and L-functions, Vol. II (Ann Arbor, MI, 1988), volume 11 of Perspect. Math., pages 143-226. Academic Press, Boston, MA, 1990.

[Kab01] A. C. Kable. The tensor product of exceptional representations on the general linear group. Ann. Sci. École Norm. Sup. (4), 34(5):741-769, 2001.

[Kap15a] E. Kaplan. The characterization of theta-distinguished representations of $G L_{n}$. arXiv:1502.06643 [math.RT], http://arxiv.org/pdf/1502.06643v1.pdf, 2015.

[Kap15b] E. Kaplan. The theta period of a cuspidal automorphic representation of GL(n). Internat. Math. Res. Notices, 2015(8):2168-2209, 2015.

[Kap16] E. Kaplan. The double cover of odd general spin groups, small representations and applications. J. Inst. Math. Jussieu, FirstView:1-63, 12016.

[KP84] D. A. Kazhdan and S. J. Patterson. Metaplectic forms. Inst. Hautes Études Sci. Publ. Math., 59(1):35-142, 1984.

[Kub67] T. Kubota. Topological covering of SL(2) over a local field. J. Math. Soc. Japan, 19(1):114-121, 1967.

[Laf02a] L. Lafforgue. Chtoucas de Drinfeld et correspondance de Langlands. Invent. Math., 147(1):1-241, 2002.

[Laf02b] L. Lafforgue. Chtoucas de Drinfeld, formule des traces d'Arthur-Selberg et correspondance de Langlands. In Proceedings of the International Congress of Mathematicians, Vol. I (Beijing, 2002), pages 383-400. Higher Ed. Press, Beijing, 2002. 
[LRS99] W. Luo, Z. Rudnick, and P. Sarnak. On the generalized Ramanujan conjecture for GL( $n)$. In Automorphic forms, automorphic representations, and arithmetic (Fort Worth, TX, 1996), volume 66 of Proc. Sympos. Pure Math., pages 301-310. Amer. Math. Soc., Providence, RI, 1999.

[LW13] J.-P. Labesse and J.-L. Waldspurger. La formule des traces tordue d'après le Friday Morning Seminar, volume 31 of CRM Monograph Series. American Mathematical Society, Providence, RI, 2013. With a foreword by Robert Langlands [dual English/French text].

[Mac95] I. G. Macdonald. Symmetric functions and Hall polynomials. Oxford University Press, New York/London, second edition, 1995.

[Mat69] H. Matsumoto. Sur les sous-groupes arithmétiques des groupes semi-simples déployés. Ann. Sci. École Norm. Sup. (4), 2(1):1-62, 1969.

[McN11] P. J. McNamara. Metaplectic Whittaker functions and crystal bases. Duke Math. J., 156(1):1-31, 2011.

[McN12] P. J. McNamara. Principal series representations of metaplectic groups over local fields. In Multiple Dirichlet series, L-functions and automorphic forms, volume 300 of Progr. Math., pages 299-327. Birkhäuser/Springer, New York, 2012.

[McN14] P. J. McNamara. The Metaplectic Casselman-Shalika Formula, 2014.arXiv:1103.4653[math.RT], http://arxiv.org/pdf/1103.4653v3.pdf, to appear in Trans. Amer. Math. Soc.

[Moo68] C. C. Moore. Group extensions of p-adic and adelic linear groups. Inst. Hautes Études Sci. Publ. Math., 35(1):157-222, 1968.

[MW95] C. Møglin and J.-L. Waldspurger. Spectral decomposition and Eisenstein series. Cambridge University Press, New York, 1995.

[Rao93] R. Rao. On some explicit formulas in the theory of Weil representations. Pacific J. Math., 157(2):335-371, 1993.

[Sak06] Y. Sakellaridis. A Casselman-Shalika formula for the Shalika model of $\mathrm{GL}_{n}$. Canad. J. Math., 58(5):1095-1120, 2006.

[Sak13] Y. Sakellaridis. Spherical functions on spherical varieties. Amer. J. Math., 135(5):1291-1381, 2013.

[Sav88] G. Savin. Local Shimura correspondence. Math. Ann., 280(2):185-190, 1988.

[Sha81] F. Shahidi. On certain L-functions. Amer. J. Math., 103(2):297-355, 1981.

[Sha88] F. Shahidi. On the Ramanujan conjecture and finiteness of poles for certain $L$-functions. Ann. of Math. (2), 127(3):547-584, 1988.

[Sha90] F. Shahidi. A proof of Langlands' conjecture on Plancherel measures; complementary series of p-adic groups. Ann. of Math., 132(2):273-330, 1990.

[Sil79] A. J. Silberger. Introduction to harmonic analysis on reductive p-adic groups. Princeton University Press and University of Tokyo Press, Princeton, New Jersey, 1979.

[Sou93] D. Soudry. Rankin-Selberg convolutions for $S O_{2 l+1} \times G L_{n}$ : local theory, volume 105, no. 500 of Mem. Amer. Math. Soc. Amer. Math. Soc., Providence, R. I., 1993.

[Sou95] D. Soudry. On the Archimedean theory of Rankin-Selberg convolutions for $S O_{2 l+1} \times G L_{n}$. Ann. Scient. Éc. Norm. Sup., 28(2):161-224, 1995.

[Sou05] D. Soudry. On Langlands functoriality from classical groups to $G L_{n}$. In J. Tilouine, H. Carayol, M. Harris, and M.-F. Vignéras, editors, Formes Automorphes (I); Actes du Semestre du CEB, Printemps 2000, volume 298 of Astérisque, pages 335-390, 2005.

[Szp09] D. Szpruch. Computation of the local coefficients for principal series representations of the metaplectic double cover of $\mathrm{SL}_{2}(\mathbb{F})$. J. Number Theory, 129(9):2180-2213, 2009.

[Tak14] S. Takeda. The twisted symmetric square L-function of GL(r). Duke Math. J., 163(1):175-266, 2014.

[Wal83] N. R. Wallach. Asymptotic expansions of generalized matrix entries of representations of real reductive groups. In Lie group representations, I (College Park, Md., 1982/1983), volume 1024 of Lecture Notes in Math., pages 287-369. Springer, Berlin, 1983.

[Wal88] N. R. Wallach. Real reductive groups. I, volume 132 of Pure and Applied Mathematics. Academic Press, Inc., Boston, MA, 1988.

[Wa192] N. R. Wallach. Real reductive groups. II, volume 132 of Pure and Applied Mathematics. Academic Press, Inc., Boston, MA, 1992. 
[Wei64] A. Weil. Sur certains groupes d'opérateurs unitaires. Acta Math., 111(1):143-211, 1964.

[Wei95] A. Weil. Basic number theory. Classics in Mathematics. Springer-Verlag, Berlin, 1995. Reprint of the second (1973) edition.

Department of Mathematics, The Ohio State University, Columbus, OH 43210, USA

E-mail address: kaplaney@gmail.com

Department Mathematik, FAU Erlangen-Nürnberg, Cauerstr. 11, 91058 Erlangen, GerMANY

E-mail address: moellers@math.fau.de 